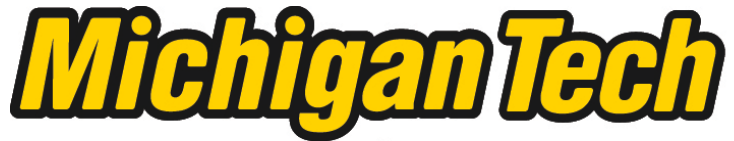 \\ Michigan Technological University Create the Future Digital Commons @ Michigan Tech
}

Dissertations, Master's Theses and Master's Reports - Open

Dissertations, Master's Theses and Master's

Reports

2008

Photolithography based patterning of bacteriorhodopsin films

Christopher M. Anton

Michigan Technological University

Follow this and additional works at: https://digitalcommons.mtu.edu/etds

Part of the Mechanical Engineering Commons

Copyright 2008 Christopher M. Anton

\section{Recommended Citation}

Anton, Christopher M., "Photolithography based patterning of bacteriorhodopsin films", Dissertation, Michigan Technological University, 2008.

https://doi.org/10.37099/mtu.dc.etds/347

Follow this and additional works at: https://digitalcommons.mtu.edu/etds

Part of the Mechanical Engineering Commons 


\title{
Photolithography Based Patterning of Bacteriorhodopsin Films
}

\author{
by \\ Christopher M. Anton

\begin{abstract}
A DISSERTATION
Submitted in partial fulfillment of the requirements

for the degree of

DOCTOR OF PHILOSOPHY

(Mechanical Engineering - Engineering Mechanics)
\end{abstract} \\ Michigan Technological University \\ 2008
}

Copyright (C) Christopher M. Anton 2008 
This dissertation, "Photolithography Based Patterning of Bacteriorhodopsin Films," is hereby approved in partial fulfillment of the requirements for the degree of DOCTOR OF PHILOSOPHY in the field of Mechanical Engineering-Engineering Mechanics.

\section{DEPARTMENT:}

Mechanical Engineering-Engineering Mechanics

Signatures:

Dissertation Advisor

Craig R. Friedrich

Department Chair

William W. Predebon

Date 


\begin{abstract}
The patterning of photoactive purple membrane (PM) films onto electronic substrates to create a biologically based light detection device was investigated. This research is part of a larger collaborative effort to develop a miniaturized toxin detection platform. This platform will utilize PM films containing the photoactive protein bacteriorhodopsin to convert light energy to electrical energy.
\end{abstract}

Following an effort to pattern PM films using focused ion beam machining, the photolithography based bacteriorhodopsin patterning technique (PBBPT) was developed. This technique utilizes conventional photolithography techniques to pattern oriented PM films onto flat substrates. After the basic patterning process was developed, studies were conducted that confirmed the photoelectric functionality of the PM films after patterning. Several process variables were studied and optimized in order to increase the pattern quality of the PM films. Optical microscopy, scanning electron microscopy, and interferometric microscopy were used to evaluate the PM films produced by the patterning technique. Patterned PM films with lateral dimensions of $15 \mu \mathrm{m}$ have been demonstrated using this technique. Unlike other patterning techniques, the PBBPT uses standard photolithographic processes that make its integration with conventional semiconductor fabrication feasible.

The final effort of this research involved integrating PM films patterned using the PBBPT with PMOS transistors. An indirect integration of PM films with PMOS transistors was successfully demonstrated. This indirect integration used the voltage produced by a patterned PM film under light exposure to modulate the gate of a PMOS transistor, activating the transistor. Following this success, a study investigating how this PM based light detection system responded to variations in light intensity supplied to the PM film. This work provides a successful proof of concept for a portion of the toxin detection platform currently under development. 


\section{Acknowledgements}

The culmination of this work represents the combined efforts of countless individuals who have helped me reach this point in my life. This accomplishment belongs in part to each of them. I would first like to thank my parents, whose love and guidance through my life has made me the person I am today. I would also like to say thank you to a very special elementary school teacher, Mrs. Carol LeGray, whose endless patience and skill helped me overcome my learning disability. Her effort, along with other teachers over the years, has given me the confidence to take on the challenges of higher learning.

I would like to thank my advisor, Dr. Craig Friedrich, for giving me the opportunity to work with him in this exciting line of research. His guidance, advice, and enthusiasm during this research project have been critical to the success of this research. He has helped to prepare for my professional career to come, while making my graduate school experience an excellent one. I could not have asked for a better advisor.

I would like to thank my committee members: Dr. Paul Bergstrom, Dr. Donald Lueking, and Dr. Michele Miller for their guidance and support during my research. Their expertise and advice has helped greatly in the success of this project. I would especially like to thank Dr. Donald Lueking for the countless hours of help and instruction he and his graduate students have put in to helping this mechanical engineer become proficient in a biology laboratory.

I would like to all of the graduate students I have had the privilege to work with for all of the help they have given me during my research. I would like to give a special thanks to Mark Griep and Karl Walczak, with whom I have worked most closely with on this project. I would also like to thank Eric Winder, Tracy Naher, Daw Don Cheam, and Manoranjan Acharya for sharing their time and expertise with me, and helping this work move forward. 
Finally, I would like to express my deepest love and thanks to my family. To my wife Kari, whose endless devotion and belief in me has given me the strength to reach this point in my life. Her countless sacrifices over the past four years have made this work possible. And also to my son Liam, who brings great joy and laughter to my life.

The research reported in this document was performed in connection with contract DAAD17-03-C-0115 with the U.S. Army Research Laboratory. The views and conclusions contained in this document are those of the authors and should not be interpreted as presenting the official policies or position, either expressed or implied, of the U.S. Army Research Laboratory or the U.S. Government unless so designated by other authorized documents. Citation of manufacturer's or trade names does not constitute an official endorsement or approval of the use thereof. The U.S. Government is authorized to reproduce and distribute reprints for Government purposes notwithstanding any copyright notation hereon. 


\section{Table of Contents}

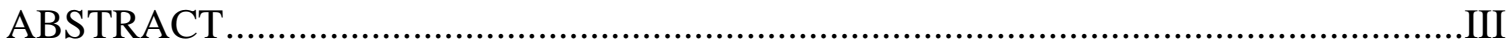

ACKNOWLEDGEMENTS .......................................................................... IV

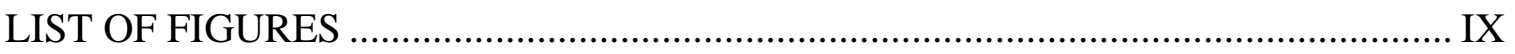

LIST OF TABLES ....................................................................................... XVI

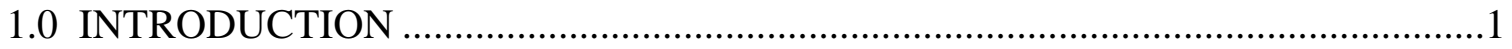

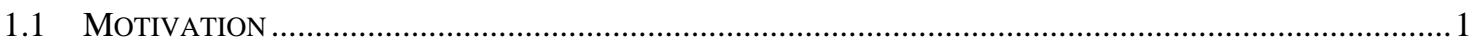

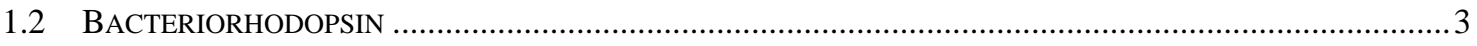

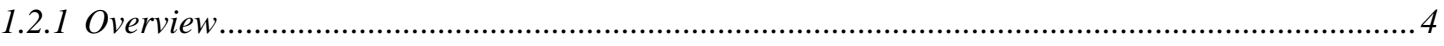

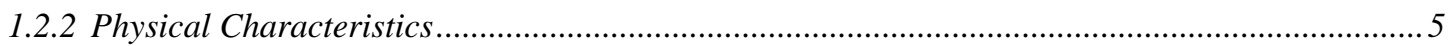

1.3 BACTERIORHODOPSIN DEPOSITION TECHNIQUES .........................................................................6

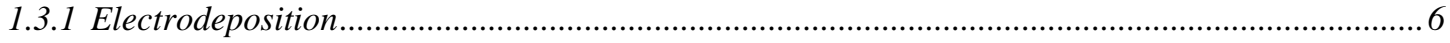

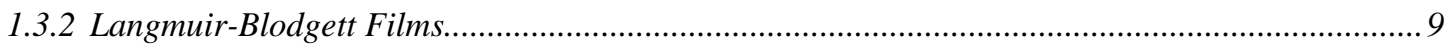

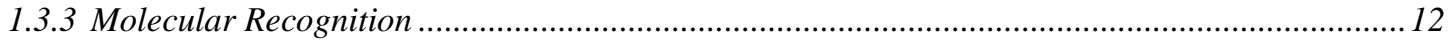

1.3.4 Laser Assisted Deposition......................................................................................................

1.3.5 Electrostatic Layer-by-Layer Deposition ...............................................................................17

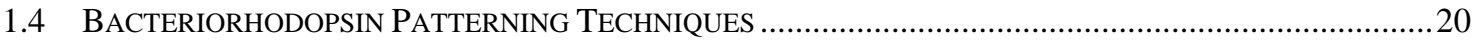

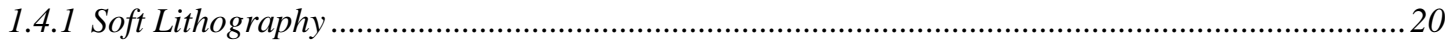

1.4.2 Electrodeposition Based Patterning …………......................................................................2

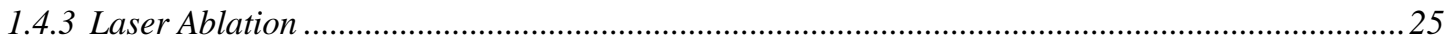

2.0 PREPARATION OF PURPLE MEMBRANE SUSPENSIONS …..........................27

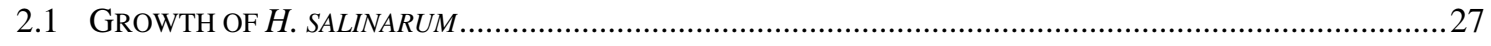

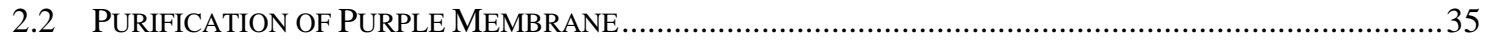

2.3 Characterization of Purified Purple Membrane .................................................................... 39 
3.1 PHYSICAL CHARACTERISTICS OF FIB MACHINEd DRIED PM FILMS …............................................... 44

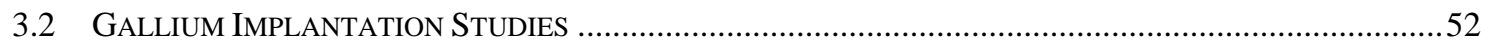

\subsection{PHOTOLITHOGRAPHY BASED BACTERIORHODOPSIN PATTERNING}

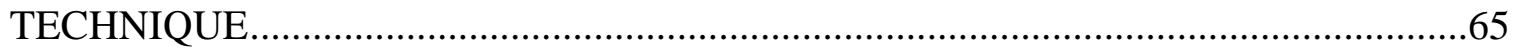

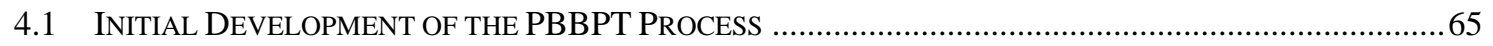

4.1.1 PBBPT Using Resist Developer to Perform Liftoff ...................................................................67

4.1.2 PBBPT Using Acetone to Perform Liftoff.................................................................................... 74

4.1.3 PBBPT Using an Acetone Sonication Bath to Perform Liftoff .................................................... 79

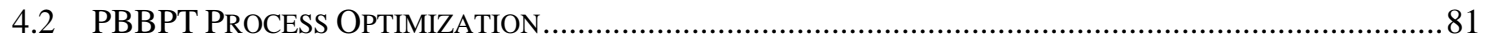

4.2.1 PM Suspension Density Optimization Study ……..................................................................... 82

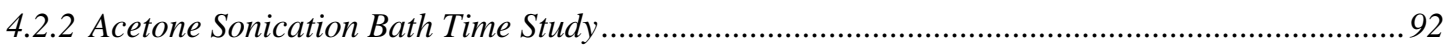

4.2.3 PВВPT Minimum Feature Size Study …………......................................................................99

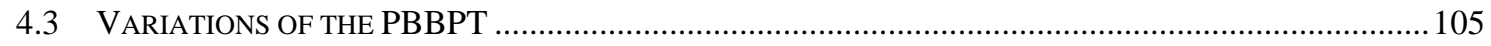

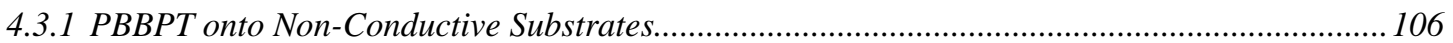

4.3.2 PBBPT with a Conductive Coating on Top of the PM Films......................................................109

4.4 CONFIRMATION OF PATTERnEd PM FiLM ELECTRICAL ACTIVITY ...................................................111

4.5 IMPACt OF ElEVATED TEMPERATURES ON PM PhOtOVOLTAGE OUTPUT .........................................117

4.6 COMPARISON WITH OTHER PATTERNING TECHNIQUES ...............................................................120

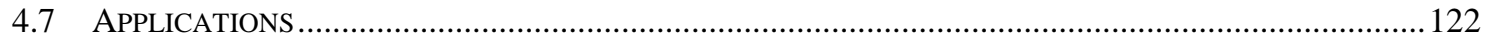

5.0 INTEGRATION OF PM FILMS WITH MOSFET TRANSISTORS.....................123

5.1 INTEGRATION OF PATTERNED PM FILMS ONTO MOSFET SUBSTRATES ...........................................124

5.2 ELIMINATING LIGHT INDUCED TRANSISTOR ACTIVATION...............................................................131

5.3 IndiRECT FunctionaLIZATION OF MOSFETs Using PATTERnEd PM FILMS..................................135 
5.4 REMOte INTEgRATION OF PATtERnEd PM FILMS With MOSFETs THROUGH IMPEDANCE

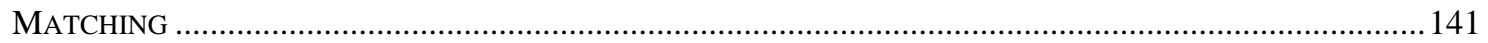

5.5 PM INTEGRATED TRANSISTOR RESPONSE VS. LIGHT INTENSITY ....................................................148

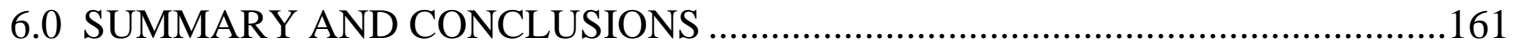

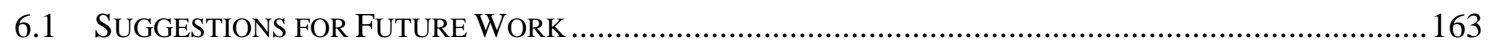

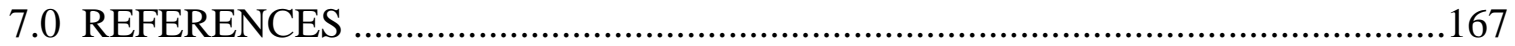




\section{List of Figures}

Figure 1: System flow chart representing the proposed toxin detection platform.............2

Figure 2: H. salinarum growth in a sidearm flask. ....................................................30

Figure 3: Bioflo III Continuous/Batch Fermentor growing H. salinarum........................32

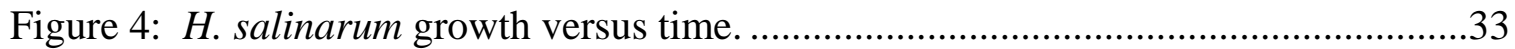

Figure 5: Flow chart describing the basic steps taken to grow and purify PM................36

Figure 6: The result of centrifuging PM through linear sucrose gradients......................38

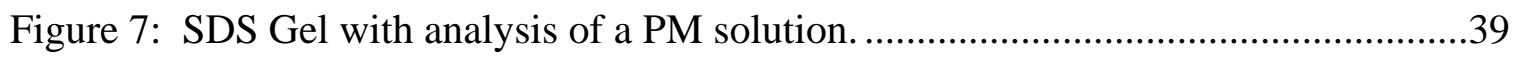

Figure 8: Absorbance spectra of a purified PM liquid suspension. ...............................41

Figure 9: FIB image of PM Aluminum interface........................................................45

Figure 10: FIB image of two parallel trenches machined in the Al-PM interface............46

Figure 11: FIB image of two parallel trenches machined in the Al-PM interface...........47

Figure 12: IFM image of two parallel trenches machined using a FIB with a M1-50

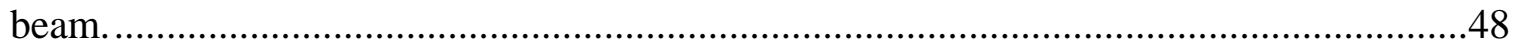

Figure 13: IFM images of two parallel trenches machined using a FIB with a M1-100

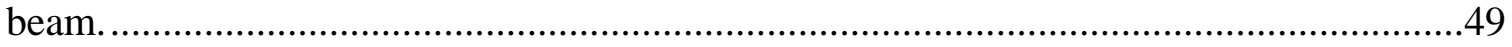

Figure 14: FE-SEM images of FIB machined PM films. …...........................................50

Figure 15: FE-SEM images of a $20 \mu \mathrm{m}$ x $20 \mu \mathrm{m}$ box FIB machined into a dried PM

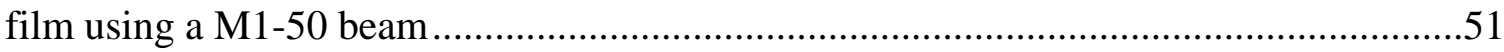


Figure 16: FE-SEM images of a $20 \mu \mathrm{m}$ x $20 \mu \mathrm{m}$ box FIB machined into a dried PM film using a M1-100 beam

Figure 17: FE-SEM images of a $40 \mu \mathrm{m}$ x $10 \mu \mathrm{m}$ trench FIB machined into a dried

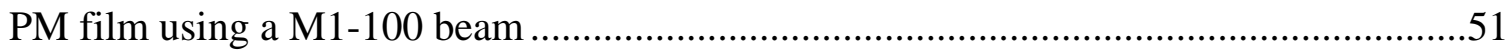

Figure 18: Illustration of gallium implantation into a silicon substrate..........................53

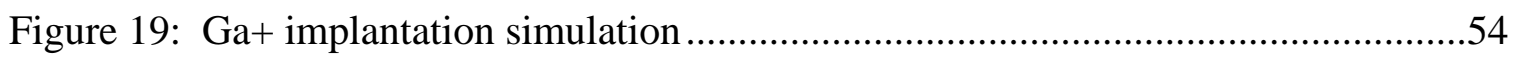

Figure 20: Raman scan performed at the approximate center of a 100 micron by 100 micron square machined in polished silicon using a FIB machine................................56

Figure 21: Intensity of Raman signal at a Raman shift of 520.7 as the scan point moves away from a corner of the 100 micron FIB machined square in polished

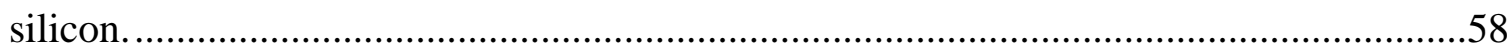

Figure 22: Raman scan of an oriented dried PM film on a brass substrate ....................61

Figure 23: Basic PBBPT process flow.................................................................66

Figure 24: Optical microscope images of oriented PM films.....................................70

Figure 25: Optical microscope images of PM film patterns $(\mathrm{OD}=6.5) \ldots \ldots \ldots \ldots \ldots \ldots \ldots \ldots \ldots . . .71$

Figure 26: Optical microscope images of PM film patterns (OD = 3.25) ......................71

Figure 27: Optical microscope images of PM film patterns $(\mathrm{OD}=0.41) \ldots \ldots \ldots \ldots \ldots \ldots \ldots . . . .72$

Figure 28: Optical microscope images of PM film patterns $(\mathrm{OD}=0.203) \ldots \ldots \ldots \ldots \ldots \ldots . . . . .72$

Figure 29: IFM analysis of the interface between the substrate surface and the

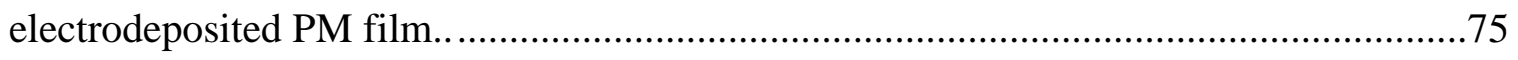

Figure 30: Optical microscope images of oriented PM films prior to photoresist strip. ..76 Figure 31: Optical microscope images of oriented PM films after photoresist strip........77 
Figure 32: IFM analysis of oriented PM films after photoresist strip...........................78

Figure 33: FE-SEM images of a single patterned PM film ........................................79

Figure 34: Optical microscope images of patterned PM films produced by the

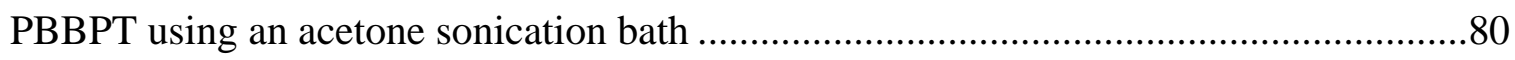

Figure 35: IFM analysis of patterned PM films produced by the PBBPT using an

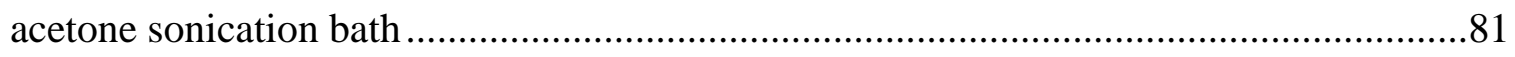

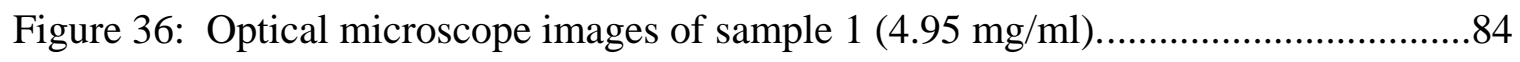

Figure 37: IFM scan of sample $1(4.95 \mathrm{mg} / \mathrm{ml})$. Maximum feature height $~ 2.6 \mu \mathrm{m} . . . . . .84$

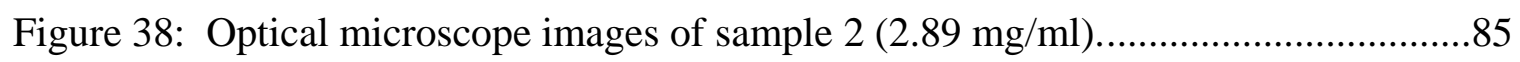

Figure 39: IFM scan of sample $2(2.89 \mathrm{mg} / \mathrm{ml})$. Maximum feature height $~ 8.3 \mu \mathrm{m} . . . . . .85$

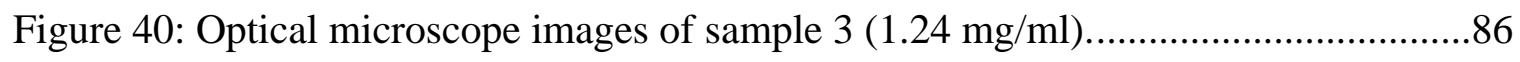

Figure 41: IFM scan of sample $3(1.24 \mathrm{mg} / \mathrm{ml})$. Maximum feature height $~ 1.4 \mu \mathrm{m} . \ldots . . .86$

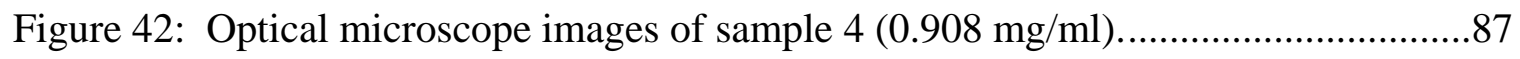

Figure 43: IFM scan of sample $4(0.908 \mathrm{mg} / \mathrm{ml})$. Maximum feature height $\sim 1.5 \mu \mathrm{m} . . . .87$

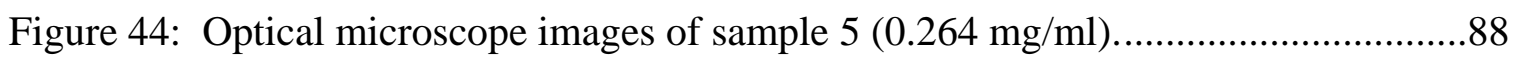

Figure 45: IFM scan of sample $5(0.264 \mathrm{mg} / \mathrm{ml})$. Maximum feature height $\sim 0.28 \mu \mathrm{m} . . .88$

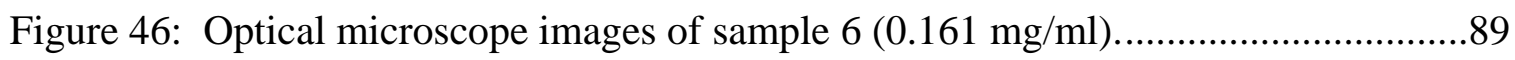

Figure 47: IFM scan of sample $6(0.161 \mathrm{mg} / \mathrm{ml})$. Maximum feature height $\sim 0.45 \mu \mathrm{m} . . .89$

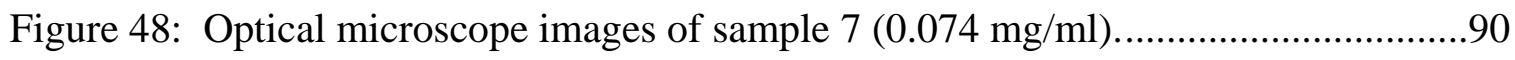

Figure 49: IFM scan of sample $7(0.074 \mathrm{mg} / \mathrm{ml})$. Maximum feature height $\sim 1.35 \mu \mathrm{m} . . .90$

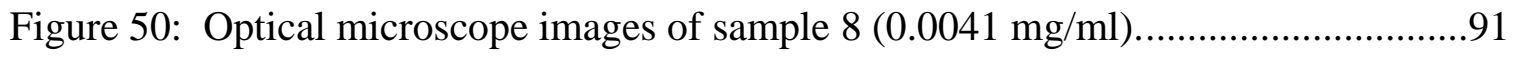

Figure 51: IFM scan of sample $8(0.0041 \mathrm{mg} / \mathrm{ml})$. Maximum feature height $~ 1.1 \mu \mathrm{m} . . .91$ 
Figure 52: Optical microscope images of sample 1 (30 sec sonication).........................94

Figure 53: IFM scan of sample 1 (30 sec sonication. ..............................................94

Figure 54: Optical microscope images of sample 2 (45 sec sonication)........................95

Figure 55: IFM scan of sample 2 (45 sec sonication)...........................................95

Figure 56: Optical microscope images of sample 3 (60 sec sonication)........................96

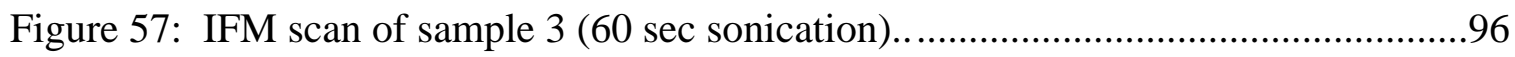

Figure 58: Optical microscope images of sample 4 (120 sec sonication)......................97

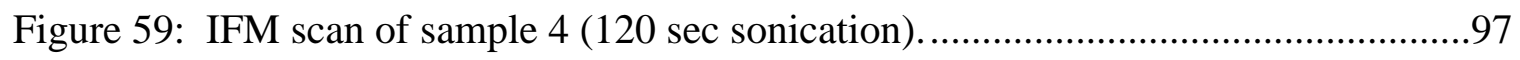

Figure 60: FE-SEM images of $60 \mu \mathrm{m}$ square patterned PM films..............................101

Figure 61: FE-SEM images of $50 \mu \mathrm{m}$ square patterned PM films ..............................101

Figure 62: FE-SEM images of $40 \mu \mathrm{m}$ square patterned PM films ..............................101

Figure 63: FE-SEM images of $40 \mu \mathrm{m}$ square patterned PM films ...............................102

Figure 64: FE-SEM images of a $30 \mu \mathrm{m}$ square patterned PM film.............................102

Figure 65: FE-SEM images of $25 \mu \mathrm{m}$ square patterned PM films ...............................102

Figure 66: FE-SEM images of $20 \mu \mathrm{m}$ square patterned PM films ..............................103

Figure 67: FE-SEM images of a damaged $20 \mu \mathrm{m}$ PM film ......................................103

Figure 68: FE-SEM images of $15 \mu \mathrm{m}$ square patterned PM films ..............................103

Figure 69: FE-SEM images of damaged $15 \mu \mathrm{m}$ PM films .........................................104

Figure 70: FE-SEM images of $10 \mu \mathrm{m}$ square patterned PM films ...............................104

Figure 71: FE-SEM images of $5 \mu \mathrm{m}$ square patterned PM films ................................104

Figure 72: Schematic representation of a revised PBBPT process flow .......................106 
Figure 73: IFM scan of a patterned PM film produced using the revised PBBPT process that allows for deposition onto non-conductive substrates. .108

Figure 74: Schematic representation of the revised PBBPT process flow.

Figure 75: DC voltage response of pre-acetone sonication bath 4 mm PM square film to pulsed LED light.

Figure 76: DC voltage response of post-acetone sonication bath $4 \mathrm{~mm}$ PM square film to pulsed LED light

Figure 77: $2.5 \mathrm{~mm}$ patterned PM square photovoltage after exposure to a $90^{\circ} \mathrm{C}$ hot plate for 120 seconds

Figure 78: Correlation between maximum un-amplified PM photovoltage and temperature

Figure 79: Schematic representation of the integration of PM films with a PMOS array using the PBBPT

Figure 80: Source current vs. time plot due to xenon light modulation of a PMOS transistor functionalized with a patterned PM film

Figure 81: Source current vs. time plot due to resistor modulation of a PMOS transistor functionalized with a patterned PM film

Figure 82: Source current vs. time plot due to xenon light modulation of a nonfunctionalized PMOS transistor

Figure 83: Schematic representation of the chrome film masking of PMOS transistor active areas. 
Figure 84: Drain current vs. time plot of a PMOS transistor exposed to two pulses of incandescent light

Figure 85: Schematic representation of the measurement setup used for the remote integration of a patterned PM film with a PMOS transistor.

Figure 86: PMOS transistor response to remote activation by a patterned PM film ......138

Figure 87: Voltage response of patterned PM film to an LED light source

Figure 88: Schematic representation of the remote integration of a patterned PM film with a PMOS transistor using impedance matching.

Figure 89: PM photovoltage due to pulsed LED light

Figure 90: PMOS transistor response to remote patterned PM film photovoltage activation 145

Figure 91: PMOS transistor response to a $30 \mathrm{mV}$ square wave run through the measurement setup .147

Figure 92: PM photovoltage due to LED illumination of $1.30175 \mathrm{E}-03 \mathrm{~W} / \mathrm{cm}^{2}$ at a distance of $68 \mathrm{~mm}$.

Figure 93: PMOS transistor response due to PM photovoltage.

Figure 94: PM photovoltage due to LED illumination of $1.15327 \mathrm{E}-05 \mathrm{~W} / \mathrm{cm}^{2}$ at a distance of $68 \mathrm{~mm}$. .151

Figure 95: PMOS transistor response due to PM photovoltage. 152

Figure 96: PM photovoltage due to LED illumination of $4.37520 \mathrm{E}-05 \mathrm{~W} / \mathrm{cm}^{2}$ at a distance of $300 \mathrm{~mm}$. .153

Figure 97: PMOS transistor response due to PM photovoltage. .154 
Figure 98: PM photovoltage due to LED illumination of $8.79365 \mathrm{E}-07 \mathrm{~W} / \mathrm{cm}^{2}$ at a

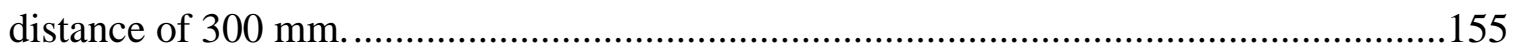

Figure 99: PMOS transistor response due to PM photovoltage...................................156

Figure 100: Approximate maximum PM photovoltage versus LED light intensity.......158

Figure 101: Approximate maximum PMOS transistor drain current response versus

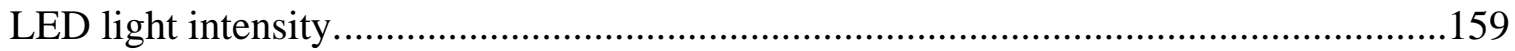




\section{List of Tables}

Table 1: Reagents required to prepare $H$. salinarium growth medium..........................28

Table 2: PM suspension densities calculated using Beer's law....................................82

Table 3: Size and spacing of squares of the PBBPT minimum feature size mask. .........99

Table 4: Acetone sonication time, pre-photoresist strip voltage, post-photoresist

voltage and change in voltage after the photoresist strip are shown............................116

Table 5: PMOS transistor response vs. LED light intensity study. ..............................157 


\subsection{Introduction}

The goal of this project is to develop a method of patterning purple membrane films containing bacteriorhodopsin onto existing complimentary metal-oxide-semiconductor (CMOS) electronic device arrays. The ability to selectively deposit purple membrane

films will allow the integration of bacteriorhodopsin and microelectronic devices to create an optical sensing platform. The patterning technique will provide a way to form high resolution patterned purple membrane films with feature sizes approaching $5 \mu \mathrm{m}$. The technique must be able to pattern the purple membrane without damaging the functionality of the bacteriorhodopsin.

\subsection{Motivation}

The ability to detect and identify substances present in the environment is a topic of great interest to the United States military. Devices capable of detecting environmental hazards are of vital importance in keeping military and civilian personnel safe, both at home and abroad. Currently, there are a wide variety of sensor platforms that are capable of detecting hazardous substances such as toxins and biohazards present in the environment. These sensor platforms range in size from bench-top systems to handheld devices.

This research is part of a collaborative effort to design and fabricate a micro-scale sensor platform capable of detecting environmental hazards. The platform will utilize microelectronics technology, biotechnology and nanotechnology to create a sensor platform with size and packaging advantages over that of the current field deployable detection devices. Our vision of the final platform is a small microchip-type device that 
could be placed on a soldier's uniform, military vehicles, or in packaged smart munitions systems. A bottom-up flow chart displaying the individual technologies used in the toxin detection platform are shown in Figure 1.

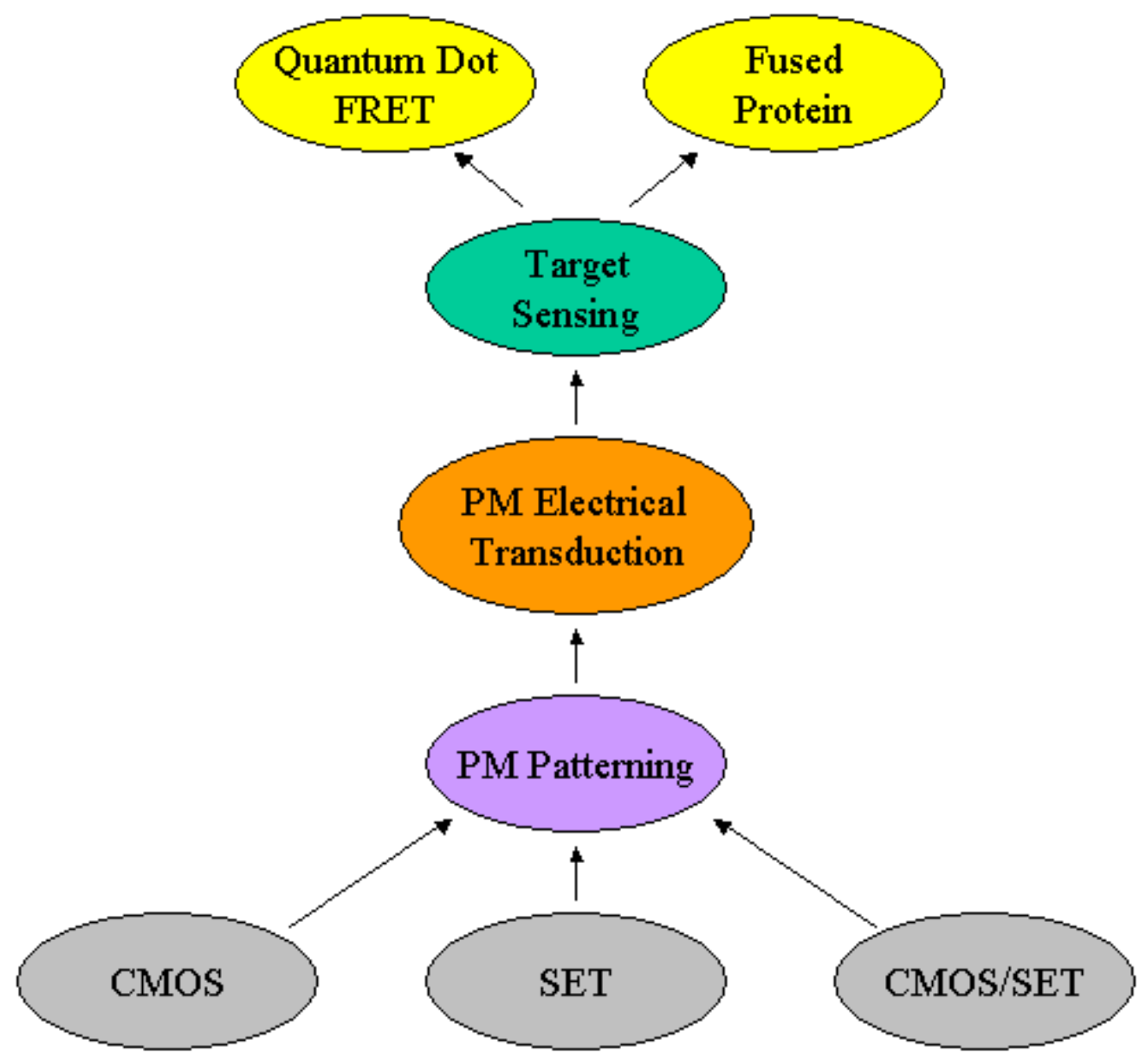

Figure 1: Bottom-up system flow chart representing the proposed toxin detection platform currently under development. This project addresses PM patterning, PM/CMOS integration and PM electrical transduction.

To create a miniaturized toxin detection platform, purple membrane (PM) films will be patterned onto microelectronic devices such as CMOS transistors or single electron 
transistors (SETs). The PM films will provide the optical to electrical transduction used to modulate the microelectronic device. To complete the system, an sensing technology using quantum dot Forster resonance energy transfer (FRET) or fused proteins will be placed on top of the PM functionalized microelectronic substrate. The receptor element will preferentially bind to a specific target, and disrupt the optical to electrical transduction of the PM film. This disruption will create a change in the electrical signal provided by the patterned PM films to the microelectronic device, causing a change in the output of the device. This change in device output indicates that the target is present in the environment. This project focuses on the development of a PM patterning technique, and the integration of patterned PM films with CMOS devices.

\subsection{Bacteriorhodopsin}

Bacteriorhodopsin (bR) is a protein capable of converting energy captured from photons of light into chemical energy, in the form of a proton gradient. When bR is irradiated with light of the appropriate wavelength (450 - $650 \mathrm{~nm})$, a proton is transported from one side of the protein to the other. If $b R$ is aligned such that all of the $b R$ molecules are oriented in the same direction, an electrical potential is created under the influence of light. This potential can then be coupled to sensitive electronics capable of indicating the change in potential of the system due to the presence of light.

This optical to electrical conversion of energy can then be used as the energy transduction mechanism of a micro-scale sensor platform. The bR produces a response both when it is exposed to a light source and when the light source is removed. This response can be used to trigger a transistor, operational amplifier, or other electronic device to provide an electrical system response to the change in light on the bR. Unlike most other light sensitive materials, bR is only sensitive to a specific light wavelength band. This can be used advantageously when designing a sensing system. bR also does not require external power to perform the optical to electrical conversion of energy, 
which is desirable when designing a miniaturized system. After this micro-scale light detection platform is developed, a secondary detection system can be built on top of the bR.

\subsubsection{Overview}

Halobacterium salinarum is an extremophile organism found in warm concentrated salt solutions with low oxygen tension [1]. These conditions are typically found in salt lakes, salt marshes, as well as desalinization plants. As a source of energy in this extreme environment, $H$. salinarum is able to capture energy from the sun and convert it into chemical energy. This photosynthetic system is unique to the halobacteria, and is much less complicated than plant and algal photosynthesis. The key component to this photocycle is the membrane protein bacteriorhodopsin. bR occurs along with some unique membrane lipids as a two-dimensional lattice and comprises the organism's purple membrane (PM). This purple membrane is integrated into the cytoplasmic membrane of the $H$. salinarum cell. When $H$ salinarum is grown chemoheterotrophically, the organism is devoid of bR in its cytoplasmic membrane. However, when $H$. salinarum is grown aerobically in the presence of light, it produces bR and inserts it into its pre-existing cytoplasmic membrane to provide energy for the organism.

When the PM is exposed to sunlight the bR in the membrane acts as a proton pump, moving protons from the intracellular side to the extracellular side of the membrane. Within each PM, the bR and its associated lipids are ordered in a crystalline hexagonal array with all of the bR molecules oriented in the same direction. PM does not require any other component from the $H$. salinarum cell to exhibit this photoresponse. This allows researchers to remove the purple membrane from the cell without compromising the PM's functionality. 


\subsubsection{Physical Characteristics}

Research has been conducted on bR since its discovery in the 1970's. Many of the initial studies focused on the physical and chemical properties of bR. Researchers were intrigued by how the bR molecule was converting light energy to chemical energy. These early efforts have provided an understanding of the bR photocycle. The bR molecule contains 261 amino acids arranged into seven $\alpha$-helical bundles. These seven bundles surround a retinylidene residue. Further discussion about the chemical structure and photocycle of bR are beyond the scope of this document, but are widely available in the literature [1].

Most recently, interest has focused on exploring many of the physical characteristics of bR, and more specifically PM. Atomic force microscopy (AFM) studies have provided information on the physical dimensions of PM patches [1,2]. AFM images in both contact mode and non-contact mode have shown that individual PM patches are irregular in size and shape, having lateral dimensions ranging from several hundred nanometers to several microns. These studies have also shown that the thickness of an individual PM patch is $5 \mathrm{~nm}$ and is quite uniform [1].

Because the bR molecule is relatively simple and is packed along with lipids in a hexagonal crystalline array, PM is a very robust membrane. Dried PM films have been shown to withstand temperatures as high as $140^{\circ} \mathrm{C}$ [3] and as low as $-85^{\circ} \mathrm{C}$ without any loss of functionality. Dried PM films have been shown to be extremely stable in an ambient environment, maintaining their photochemical functionality for years after deposition [4]. The robust nature of PM makes it an ideal biological material for integration with microelectronics to create an environmental sensing platform. 


\subsection{Bacteriorhodopsin Deposition Techniques}

In order to integrate PM films containing bR into an electrical device, techniques must be developed for depositing PM films. For PM films to produce an electrical response to light detectible by an electronic device, the PM patches in the film must be oriented with respect to one another. The PM patches will have a response to light regardless of their orientation, but the response is vectorial in nature. If a film of PM is randomly oriented, the net electrical response will be very small. Only if the majority of the PM patches are oriented in the same direction can a usable electrical response be achieved.

\subsubsection{Electrodeposition}

Electrodeposition is a process in which an electric field assists in the alignment and deposition of a material suspended in a liquid medium. Electrodeposition has been widely used as a method of creating oriented PM films on conductive substrates. Typically a drop of liquid, usually water, containing PM patches is placed on a conductive substrate that acts as the positive electrode in the experimental setup. A second conductive substrate, which acts as the negative electrode, is then brought down on top of the PM suspension so that contact is made between the liquid and the negative electrode. A voltage is then applied between the positive and negative electrodes. This voltage creates an electric field in the liquid suspension. bR molecules have weak dipole moments that cause them to align in the direction of an electric field. The cytoplasmic side of PM patches also carries a net negative charge at a neutral $\mathrm{pH}$, which allows for deposition onto the positive electrode to occur.

After a field is applied, the PM patches align with the field and are pulled towards the positive electrode. PM patches that reach the electrode are bound to the electrode. The 
gap between the two electrodes, the voltage applied, the amount of time the voltage is applied, and the concentration of the PM suspension all impact the effectiveness of the deposition, as well as the amount of PM deposited. The applied electric field is typically in the range of 20-40 V/cm. If the electric field is too low, the PM patches will not bind to the positive electrode. If the electric field is too high, the PM patches may lose their functionality. The gap between the two electrodes is typically $1-2 \mathrm{~mm}$. The electrode gap can only be as large as the height of the PM suspension above the surface of the electrode since contact between the top electrode and the suspension is required. Deposition times can range from tens-of-seconds to minutes and the concentration of the PM suspension can be varied. All four of these variables contribute to the effectiveness of a PM deposition. The thickness of the PM deposition is typically controlled by changing the concentration of the PM in the liquid suspension, but the other variables in the deposition process also play roles in the final PM film thickness.

Not all of the PM in a suspension will be bound to the positive electrode. Some PM fragments will remain suspended in the liquid. This unbound PM can be washed away to maximize the degree of orientation of the firmly deposited film. After the electric field is removed, any unbound PM fragments will again become randomly dispersed in the liquid. If these fragments are allowed to dry along with the oriented deposition, the overall degree of orientation will be decreased. Submerging the electrode in distilled deionized (ddi) water and gently agitating the electrode by hand removes unbound PM fragments. The electrode is then allowed to dry, creating a dried, oriented PM film.

Wang et al. [5] studied the photocycle of dried PM films both theoretically and experimentally through electrodeposited, dried PM films. Theoretical modeling of the photocycle of dried PM films was discussed in detail. Experimental work was conducted to verify the theoretical models. PM fragments suspended in deionized water were sonicated for $5 \mathrm{~s}$ and then injected between two ITO coated electrodes separated by a 1 mm plastic spacer. An electric field of $30 \mathrm{~V} / \mathrm{cm}$ was applied for 5 minutes, causing the 
PM to be deposited onto the positive electrode. The film was dried overnight in a humidity controlled box. The resulting PM film was $5 \mathrm{~mm}$ in diameter and $30 \mu \mathrm{m}$ thick. A second ITO plate was placed on top of the PM film, and acted as a second electrode to obtain photovoltage measurements. Photovoltage vs. time plots were obtained through a custom operational amplifier circuit, and the resulting curves were found to be in good agreement with the theoretical predictions for photovoltage response of the PM film. It was also found through experimentation that increasing the impedance of instrumentation increased the measured photoresponse amplitude.

$\mathrm{Xu}$ et al. [6] used electrodeposited PM films to obtain direct measurements of the photoelectric response time of bR. A PM film was deposited onto an ITO coated glass plate by electrodeposition to form an oriented PM film. The film was then brought into contact with a BGO (Bismuth-Germanium-Oxide) crystal. The BGO crystal was used to detect the photoresponse of the PM film. A second ITO coated glass plate was placed on top of the BGO crystal, and the two ITO surfaces were connected to ensure that the entire voltage differential generated by excitation of the PM film was passed through the BGO crystal. A $1060 \mathrm{~nm}$ wavelength laser light source was used to illuminate the PM film with $500 \mathrm{fs}$ pulses of light at an energy of $25 \mu \mathrm{J} /$ pulse. This experimental setup was used to investigate various photoelectric response properties of electrodeposited PM films.

Electrodeposition has several unique aspects that make it a desirable deposition method for some applications. Electrodeposition is an simple process to perform, and requires no specialized equipment. All that is needed to carry out a deposition is two conductive substrates, the material one wishes to deposit and a voltage source that can be used to create the electric field between the two conductors. Electrodeposition is also a fast process that is capable of creating thick PM films containing many individual layers of PM in a matter of minutes. This is advantageous if one wishes to study the electrical response of PM films because the thickness of the film plays a major role in the amount of electrical activity the film will produce. 
Electrodeposition is not a perfect process. There are several shortcomings to this process that make it unsuitable for many applications. The first of these shortcomings is the controllability of the process and the reproducibility of the results. It is very difficult to fully control the thickness of the PM film that is deposited. Electrodeposition involves several variables discussed above that all interact to impact the final result. A second disadvantage of this process is that it requires the substrate below the PM film not only to be conductive, but also to be electrically accessible in order to create the electric field. To use this method to deposit PM onto the gate of a transistor, the transistor gate would have to be accessed in order to create the required electric field.

\subsubsection{Langmuir-Blodgett Films}

The Langmuir-Blodgett (LB) film technique allows the deposition of a single monolayer of a given material onto a solid substrate. If polar molecules having both hydrophobic and hydrophilic ends (an amphiphilic material) are placed on top of a liquid, a single monolayer of molecules can be formed at the air-liquid interface. This monolayer may then be transferred to a solid substrate by dipping the substrate into the liquid. By this method it is possible to create multiple layers of molecules in a controlled manner.

Previous studies have shown [7] that PM can be suspended on top of a liquid to form stable monolayers. A Langmuir-Blodgett trough is commonly used to create these monolayers. LB troughs contain a well in which a liquid subphase is placed. PM is placed on top of the subphase and allowed to stabilize into a monolayer provided an appropriate concentration of PM is deposited. The LB trough is capable of monitoring and automatically adjusting the surface tension of the monolayer while simultaneously dipping a solid substrate in and out of the liquid. This allows the deposition of 
monolayers onto a solid substrate while maintaining a stable monolayer film on top of the subphase.

The LB deposition technique was used by Wang et al. [8] to fabricate a PM based photocell. A solution of $\mathrm{H}_{2} \mathrm{O}: n$-hexane: $\mathrm{N}, \mathrm{N}$-dimethylformamide (DMF) was prepared at a ratio of 0.5:1:0.2 and used as the spreading solvent. PM was added to the spreading solvent to create a PM concentration of $0.5 \mathrm{mg} / \mathrm{mL}$ in the spreading solvent. The solution was sonicated for 25 seconds to emulsify the PM fragments and then spread on the surface of a $1 \mathrm{mM} \mathrm{CdCl}_{2}$ subphase and allowed to stabilize for 30 minutes. An indiumtin-oxide (ITO) coated glass plate measuring $12 \mathrm{~mm} \times 45 \mathrm{~mm}$ with a hydrophilic surface was dipped vertically using a Face membrane balance. The transfer ratio was approximately 0.8 and the dipping speed was $20 \mathrm{~mm} / \mathrm{min}$.

After the film deposition was completed, a wet photocell was constructed. An ITO coated glass plate acted as a bottom electrode while the PM coated ITO plate was used as the top electrode. Teflon spacers provided a gap of $0.5 \mathrm{~mm}$ between the two electrodes. The volume between the two plates was filled with an aqueous electrolyte agar gel saturated with KCL at a concentration of $1 \mathrm{~mol} / \mathrm{l}$ and a $\mathrm{pH}$ between 7 and 8 . After the PM photocell was fabricated, it was connected to a Keithley Model 195 digital voltmeter, as well as an oscilloscope. The photocell was irradiated using a $500 \mathrm{~W}$ xenon lamp at a wavelength of $560 \mathrm{~nm}$ at varying intensities. Experiments were conducted to determine the photoelectric response of the photocell under a variety of lighting conditions. A linear relationship between light intensity and the resulting photocurrent was observed.

Ikonen et al. [9] studied the photoresponse obtained from PM films deposited under various LB conditions. ITO coated quartz slides were used as substrates, and a KSV 5000 LB film deposition system was used to deposit the monolayers. Ten monolayers of stearic acid ( $\mathrm{C}_{18}$ fatty acid) were deposited onto the ITO surface, followed by a PM suspension in soya-phosphatidylcholine (soya-PC) at a ratio of 7:1. PM monolayers were 
deposited in two orientations, with the extracellular side of the bR molecules oriented towards or away from the ITO surface. Multiple monolayers of PM were deposited to create a series of electrochemical cells used for testing. Once the PM layers were deposited, ten additional layers of stearic acid were added, followed by a top electrode made of InGa liquid metal. The stearic acid acted as an insulator to ensure that the electrochemical cells would not be self-grounded. The electrochemical cells produced a photovoltage when exposed to laser light at a wavelength of $570 \mathrm{~nm}$. A linear trend was observed between the number of layers of PM deposited and the induced photovoltage when exposed to the laser light. Equal but opposite photovoltage amplitudes were observed when comparing the two PM film orientations explored in this research.

Miyasaka and Koyama [10] fabricated an artificial photosensor using an LB deposited PM film. The substrate was a glass plate with 256 individual square pixels (1.3 $\mathrm{mm} \times 1.3 \mathrm{~mm}$ ) of $100 \mathrm{~nm}$ thick ITO. Each pixel was individually wired to connection pads at the outer perimeter of the pixel array. The total size of the pixel array was $31 \mathrm{~mm}$ x $31 \mathrm{~mm}$. PM monolayers were deposited across the entire substrate using the LB deposition method. A total of 10 to 14 monolayers were deposited on the substrate. A $300 \mathrm{~mm}$ spacer was placed around the pixel array on the bottom electrode, and the volume inside the spacer was filled with an electrolyte gel consisting of $4 \%$ carboxymethylchitin in $2 \mathrm{M}$ potassium chloride. A gold coated glass plate was placed on top of the spacer to act as the counterelectrode, and the entire structure was sealed with epoxy. Each pixel's photoelectric response was amplified and directed to an individual LED on a $10 \mathrm{~cm} \times 10 \mathrm{~cm}, 256$ LED array. The photosensor was then exposed to a variety of moving and flashing images, which resulted in a response from individual pixels. The various image tests performed on the photosensor demonstrated its ability to perform mobile image extraction and edge detection without the aid of operational and computational circuits. 
As with electrodeposition, the LB film method has advantages as well as disadvantages. This process is precise and reproducible, in that the exact number of monolayers deposited onto a substrate can be controlled. This high level of precision comes at the cost of time and difficulty of the process. The process of depositing monolayers is slow, requiring many hours to deposit multiple monolayers. The LB trough system can take hours to equalize after a material is deposited on top of the subphase. Cleanliness and environmental isolation are important if this process is to be successful. Dust particles falling from the air, vibrations from the room, as well as air currents in the room, are all capable of disrupting the fragile monolayer and ruining the deposition. The LB film process is also not a selective deposition process. The LB process will coat the entire substrate with the monolayer. If patterning of the substrate is required, additional steps must be taken to selectively remove the monolayer to create a pattern.

\subsubsection{Molecular Recognition}

Molecular recognition is a deposition technique that can be used to attach PM patches to a solid support, and to orient the PM patches. The most commonly used molecular recognition technique for depositing PM is the biotin/streptavidin technique. With this technique, biotin, also known as vitamin $\mathrm{H}_{\text {or }} \mathrm{B}_{7}$, is bound to lysine amino acid residues available in individual bR molecules in a process called biotinylation. It has been shown that under alkaline conditions, $(\mathrm{pH}$ 8.5-9.0) only a single lysine group located on the extracellular surface of each bR molecule is available for biotinylation [11].

Biotin must also be attached to the surface of a substrate. This is typically done by depositing a monolayer containing both biotin and lipids onto the substrate using the LB technique. After the biotin layer has been deposited, the substrate is exposed to a solution containing the protein streptavidin, and allowed to react for 30-60 minutes. 
During this reaction, streptavidin binds with the biotin already attached to the substrate surface. The binding affinity between biotin and streptavidin is so strong that the strength of the bond approaches that of a covalent bond. Streptavidin has four individual binding sites that will accept individual biotin molecules. After the surface of the substrate is functionalized with both biotin and streptavidin, a suspension of biotinylated PM is introduced to the surface and allowed to react. Again, due to the binding affinity between biotin and streptavidin, the biotin previously attached to the bR will bind with the exposed receptor sites on the streptavidin protein. The end result is an oriented layer of PM that is strongly bound to the substrate through molecular binding.

Chen et al. [12] reported using the molecular recognition technique to deposit oriented PM films onto a gold substrate. A gold coated glass slide was first functionalized by depositing a mixture of biotin-X DHPE and DMPC (1:10 molar ratio) onto the gold using the LB method. This deposition served to coat the gold surface with biotin. The functionalized surface was then allowed to react first with streptavidin, and then with biotinylated PM fragments to form an oriented PM film. Surface plasmon resonance (SPR) was used to determine that the surface mass density of streptavidin adsorbed onto the substrate was $0.46 \mathrm{ng} / \mathrm{mm}^{2}$, and an approximate ratio of streptavidin to bR molecules was 1:2. Photovoltage studies of the oriented PM film were also performed, and a peak output of $157.2 \mathrm{mV}$ was obtained for the oriented film. The photoresponse of a non-oriented PM film was also tested, and was found to be $7.8 \mathrm{mV}$. Finally, the degree of orientation of the PM film was analyzed using SPR techniques, and was found to be at least $81 \%$.

Ren et al. [13] constructed a nanomechanical light sensitive device using PM films deposited by molecular recognition. The gold surface of an AFM cantilever was first coated with cysteamine, and then with aminoalkanethiols to attach amino groups onto the gold surface. The cantilever was then biotinylated, exposed to streptavidin, and finally biotinylated PM, resulting in a PM film formed on the cantilever. The PM coated 
cantilever was placed into the head of a SPM6800 atomic force microscope, and the cantilever was immersed in an electrolyte buffer. Cantilever deflection data was captured while the PM was exposed to a modulated light source. The experiment resulted in a deflection of approximately $45 \mathrm{~nm}$ in the tip when the light source was on, and a zero deflection when the light source was turned off. It was also found that increasing the electrolyte concentration $(\mathrm{KCl})$ decreased the deflection due to light modulation.

Molecular recognition provides a powerful tool for the formation of highly ordered monolayers of PM onto a solid substrate. Because of the high binding affinity of biotin to streptavidin, this deposition method provides a strong bond between the PM patches and the substrate. This method also allows the distance between the substrate and the PM patches to be controlled using different crosslinkers attached to the biotin molecules. Like the LB method, the disadvantage of this deposition method is the time it takes to perform the deposition process. Several hours are typically required to allow the various reactions to take place. Often an LB trough is used to deposit the initial monolayer of biotin onto the substrate surface, so the limitations of the LB process are also imposed upon this deposition process. To date, only single monolayers of PM have been deposited using the molecular recognition method. It may be possible to deposit multiple monolayers of PM using this method, but no successful work in this area has been presented in the literature.

\subsubsection{Laser Assisted Deposition}

Laser Assisted Deposition (LAD) or Pulsed Laser Deposition (PLD) is a technique that allows the deposition of a thin film of target material onto a substrate. In this process, a high power laser is focused onto a target comprised of the material to be

deposited. The target is vaporized by the laser beam, forming a plume. The evaporated plume is comprised of a mixture of energetic species including atoms, molecules, 
electrons, ions, clusters, micron-sized solid particulates, and molten globulets [14]. Some portion of the evaporated plume makes contact with the substrate, and quickly cools to form a thin film comprised mainly of the target material. This process is typically performed under vacuum to facilitate plume formation as well as reduce the amount of contaminants introduced to the substrate during the deposition process. A reactive gas can also be introduced to the plume to help facilitate the deposition process.

Agarwal and Phadke [15] have reported using LAD to deposit bR onto a substrate. A PM suspension was incorporated in the lipid L- $\alpha$ - distearoylphosphatidylcholine (DSPC) at a ratio of 1:50 wt/wt using the standard method of forming lyposomes described by Szoka et al [16]. The DSPC-PM was then evenly spread onto a stainless steel disc and allowed to dry in a desiccator. This deposition process was repeated several times in order to obtain a dried film of DSPC-PM greater than $0.5 \mathrm{~mm}$ thick. This film served as the target and both glass and quartz were used as substrates in the LAD process. The process was performed in a small chamber maintained at a vacuum of $10^{-3}$ atm. The target-substrate distance was maintained at $2 \mathrm{~cm}$, and the target was rotated during exposure to the laser in order to avoid constant laser exposure to any single area of the target. A nanosecond pulsed Lambda Physik LPX 200 excimer laser was used at a wavelength of $305 \mathrm{~nm}$. The incident energy of the laser beam was varied from 5 to 12 $\mathrm{mJ}$ per pulse, with the best results seen at an incident energy of $10 \mathrm{~mJ}$ per pulse. The laser spot size was set to $0.5 \mathrm{~mm}$. A repetition rate of $5 \mathrm{~Hz}$ yielded the best deposition rates, without adversely affecting the target material.

After the LAD process was completed, the resulting thin films were analyzed using a Shimadzu UV 2100 double beam spectrophotometer, a Leitz Aristomet optical microscope, and a JEOL JDX 8030 x-ray diffractometer. Absorption spectra were captured for LAD deposited PM films on quartz both before and after subjecting the sample to ether vapors for 30 minutes. The pre-ether scan showed a strong absorption peak at $570 \mathrm{~nm}$, characteristic of the "B" state of the bR photocycle. The post-ether scan 
showed a decrease in the $570 \mathrm{~nm}$ peak and the formation of a peak at $490 \mathrm{~nm}$. This second peak was due to the formation of the 460 to $490 \mathrm{~nm}$ complex in bR in the presence of ether [17]. Agarwal and Phadke [15] reported that based on these absorbance studies, the bR retained its functionality after LAD. A second set of LAD deposition studies were conducted using PM as the target without the DSPC matrix. The resulting films did not show the absorbance characteristics typical of PM films, indicating that the PM had been damaged in the process. This study confirmed the importance of the DSPC matrix when depositing PM using LAD.

There are several key advantages of the LAD process over more conventional PM deposition techniques. The LAD process is free of solvent-solute and solute-substrate interactions. Additionally, LAD offers a technique in which preformed assemblies, such as the DSCP-PM matrix discussed above, can be lifted and deposited onto a solid surface. Despite these favorable characteristics, several aspects of the LAD process make it an undesirable deposition method for the applications proposed in this document. One drawback to this deposition method is that the PM fragments are deposited in a nonoriented fashion. A second disadvantage inherent to the LAD process is the difficulty of scaling up to large area depositions due to the narrow forward angular distribution of the plume [14]. More studies must be completed on the LAD of PM films in order to confirm that the electrical functionality of the PM film is not degraded or eliminated by the deposition process. While the absorption studies discussed by above provide some evidence that the PM survived the deposition process, further investigation of the electrical response of PM films to light must be performed to confirm that the PM films have not been altered or damaged. 


\subsubsection{Electrostatic Layer-by-Layer Deposition}

Electrostatic layer-by-layer deposition was introduced by Decher et al. [18]. In Decher's work, alternating layers of polycations and polyanions were adsorbed onto a solid substrate, resulting in films similar in molecular architecture and thickness control to LB films [19]. In order to build multilayer films using electrostatic layer-by-layer deposition, a substrate is first immersed in a vessel containing a polycationic or polyanionic solution for a given period of time. The substrate is then removed from the vessel and washed with water of the same $\mathrm{pH}$ as the polymeric solution and dried. The substrate is then immersed in the second polymeric solution for some period of time. While immersed, a single layer of the second polymeric solution is adsorbed onto the substrate. The wafer is again removed, washed, and dried. The process can be repeated to achieve the desired number of layers on the substrate surface. No limitations have been observed with respect to the number of layers that can be deposited using this method [19].

Electrostatic layer-by-layer deposition of oriented bR/polycation multilayers was reported in several papers by He et al. [20, 21]. In He's initial study, the accuracy and reproducibility of the deposition process was characterized. PM was isolated from Halobacteruml halobium R1M1 strain as described by Oesterhelt and Stoeckenius [22]. For the deposition, the PM suspension was used at a concentration of $0.5 \mathrm{mg} / \mathrm{mL}$, and the $\mathrm{pH}$ was brought to 9.4 using $0.1 \mathrm{M} \mathrm{NaCl}$. Poly-(dimethyldiallylammonium chloride) (PDAC, medium molecular weight, $20 \mathrm{wt} \%$ in water) from Aldrich Chemical Co. was used as the polycation without further purification. This polyelectrolyte was dissolved in ddi water at a concentration of $2.0 \mathrm{mg} / \mathrm{mL}$ containing $0.5 \mathrm{M} \mathrm{NaCl}$ and a $\mathrm{pH}$ of 6.8.

The polycation/PM films were deposited onto the following substrates: quartz slides for UV-vis absorption measurements, glass slides for ellipsometry and SHG analysis, and silicon wafers for AFM analysis. All substrfates were immersed in an ultrasonic bath at 
$25 \mathrm{C}$ in a mixture of ethanol/acetone/chloroform (2/1/1, v/v/v) for 30 minutes, and again in $2 \% \mathrm{KOH}$ aqueous solution under the same conditions. The substrates were then rinsed and stored in ddi water. This pretreatment procedure partially hydrolyzed the substrate surfaces and rendered a net negative charge on the surface of the substrates to facilitate polycation adsorption onto the substrate.

After the PM suspension, the polyelectrolyte and the substrates were prepared. A substrate having a negative surface charge due to the pretreatment procedure was immersed in the PDAC polyelectrolyte solution for 5 minutes. The substrate was then removed, washed in Milli-Q water for 2 minutes, and dried using compressed nitrogen. The substrate was immersed in the PM suspension discussed previously for 5 minutes, rinsed in Milli-Q water for 2 minutes, and dried with nitrogen. This process was repeated until the desired number of bilayers was obtained.

To confirm that each deposition sequence was adding a single bilayer, both a thickness study and an absorbance study were performed. The thickness study was performed using a Rudolph Research/Auto EL III ellipsometer. Film thickness measurements were taken after each bilayer, and showed a linear increase in total film thickness as the number of bilayers was increased. A thickness of $5.5 \mathrm{~nm}$ per bilayer was observed. The known thickness of a single PM layer ranges between 4.5-5.0 nm, and the thickness of a PDAC monolayer is approximately $1.0 \mathrm{~nm}$. The UV-vis absorption spectra analysis was performed using a Perkin-Elmer Lambda-9 UV/vis/near-infrared spectrophotometer. The absorbance at both $278 \mathrm{~nm}$ and $563 \mathrm{~nm}$ were shown to increase proportionally with the addition of each bilayer.

He et al. [23] also investigated the photoelectric properties of PM films deposited by the electrostatic layer-by-layer method. In this study, both a wild-type (WT) bR sample and bR from a D96N mutant were studied. The details of the deposition process were identical to the process described in the preceding publication. The experimental setup used to measure the photoelectric response of deposited PM films used a $50 \mathrm{~W}$ halogen 
lamp filtered by a long-pass, heat absorbing filter which provided light to the PM film from $530 \mathrm{~nm}$ to $750 \mathrm{~nm}$. An ITO coated glass plate ( $35 \mathrm{~mm}$ x $12 \mathrm{~mm}$ x $1 \mathrm{~mm}$ ) served as the substrate onto which the PDAC/bR multilayers were deposited. The measurements were taken in an optical glass electrochemical cell, with the ITO plate serving as the working electrode and a Pt wire $1 \mathrm{~mm}$ in diameter serving as the counter electrode. The two electrodes were separated in the electrochemical cell by $1.5 \mathrm{~cm}$, and the cell was filled with $80 \mathrm{~mL}$ of $0.5 \mathrm{M} \mathrm{KCl}$ aqueous solution acting as the electrolyte. Photoelectric signal measurements were taken of both wild-type and D96N mutant bR electrodes. Measurements were taken while changing the number of bilayers, the $\mathrm{pH}$ of the electrolyte solution, and the concentration of sodium azide contained in the electrolyte solution. The optimum $\mathrm{pH}$ of the electrolyte solution was found to be 8.5 for WT bR and 7.0 for the D96N strain of bR. Increasing the sodium azide concentration in the electrolyte increased the light-on photocurrent from $45 \mathrm{nA} / \mathrm{cm}^{2}$ at a concentration of 0 $\mathrm{mM}$, to $88 \mathrm{nA} / \mathrm{cm}^{2}$ at a concentration of $100 \mathrm{mM}$, and had no further impact at concentrations above $100 \mathrm{mM}$. The maximum light-on photocurrent achieved by the wild type PM was $55 \mathrm{nA} / \mathrm{cm}^{2}$ from 8 bilayers while the maximum photocurrent achieved by the D96N mutant was $80 \mathrm{nA} / \mathrm{cm}^{2}$ from 6 bilayers.

The photovoltaic response of electrostatic layer-by-layer deposited PM films have been investigated by Li et al. [24]. This experiment followed the same deposition procedure developed by $\mathrm{He}$ et al. [20, 21]. An ITO coated quartz plate was used as the substrate. PDAC/bR bilayers were deposited between two apomembrane (AM) layers, which helped to prevent short circuiting of the cell. A frequency doubled Nd:YAG laser at a wavelength of $532 \mathrm{~nm}$ was used to excite the bR. The magnitude of the voltage response increased under illumination by the laser source to a maximum value at approximately $160 \mu \mathrm{s}$. A linear increase in the maximum photovoltage was observed as the number of PDAC/bR bilayers was increased.

Electrostatic layer-by-layer deposition provides a controllable method for depositing oriented monolayers of PM without the use of expensive and specialized equipment. If 
performed correctly, it is possible to deposit a known number of PM monolayers onto a substrate. As with the LB deposition method, this method is a slow process requiring hours to deposit multiple PM monolayers. However, unlike the automated LB deposition method, this method requires the user to be present in the lab to perform all of the adsorption and washing steps required to complete the process.

\subsection{Bacteriorhodopsin Patterning Techniques}

To integrate PM into an electrical system requires a technique to pattern the PM. It is important to deposit oriented PM films selectively in order for the PM films to perform as a light sensitive material without impairing the functionality of the underlying electronics used to monitor the PM activity. The majority of studies performed on PM films have not attempted to create microscale patterns on a substrate. In most cases, researchers were interested in various response properties of PM films, and therefore did not attempt to pattern the film. However, several techniques for patterning PM films have already been developed and reported in the literature.

\subsubsection{Soft Lithography}

Soft lithography refers to a series of processes using elastomeric materials that produce features on a substrate. These processes are pattern replication methods, meaning that a pattern made from a hard and durable material such as a metal or silicon is used as a master. The elastomeric material is then cast on top of the master, creating a negative of the master. The elastomeric stamp can be "inked" with the material to be patterned and brought into contact with a substrate to make a pattern.

Crittenden et al. [25] described the patterning of PM films by soft lithography and electrophoretic sedimentation. This technique used a polydimethylsiloxane (PDMS) pad 
with four channels molded into the pad. The patterned side of the pad was coated with 70 $\mathrm{nm}$ of gold to create a conductive surface on top of the insulating pad. The deposited gold was left in the channels and removed from the areas of the pad that would make contact with the substrate. The PDMS pad was brought into contact with an ITO coated glass slide and uniform pressure was applied to the pad to ensure that good contact was made between the substrate and the pad. A PM suspension in distilled water, having a concentration of $5 \mathrm{mg} / \mathrm{mL}$, was injected into the four channels. After the channels were filled, a DC potential of 3 to 5 volts was applied between the ITO slide and the gold coated portion of the PDMS stamp for times ranging from 5 seconds to 30 seconds. This caused the PM fragments to be drawn to the ITO surface and form an oriented, patterned film. After applying the electric field, the pad was removed and the ITO coated glass slide was rinsed with ddi water 4 times and allowed to dry. The silicon master used to cast the PDMS pads was comprised of a grid of $5 \mu \mathrm{m}$ wide channels that formed $10 \mu \mathrm{m}$ X $10 \mu \mathrm{m}$ squares. Good conformity was observed between the silicon master and the pattern of deposited PM produced using the PDMS stamp. While the pattern reproduction on the ITO coated glass slide appeared good, a measurable electrical response could not be obtained from the patterned $b R$ when irradiated with light. The group hypothesized that the $\mathrm{bR}$ was not thick enough to produce a measurable response.

While published results concerning soft lithographic patterning of PM films is limited, work on patterning other biological substances using this method has been performed. Patterning techniques performed on other biological materials may be applicable to the patterning of PM films. Fang et al. [26] discuss a soft lithography process used to obtain submicrometer patterning of self assembled monolayers of hemoglobin on an ITO coated surface. Two possible process flows used to pattern the hemoglobin were reported. Soft lithography as well as conventional lithography was used in one process flow, while only soft lithography was used in the second process flow. A resulting hemoglobin pattern obtained through soft lithography was shown and 
discussed. These results demonstrate that this soft lithography process flow is capable of patterning biological materials with pattern feature sizes below one micrometer.

Hyun and Chilkoti [27] reported patterning biomolecules using wells formed in a PDMS stamp. An N-hydroxysuccinimide-Ester-functionalized surface was first created on the substrate surface. A solution containing biotin was placed into the PDMS wells and the substrate was brought into contact with the PDMS stamp. After the PDMS stamp was in good contact with the substrate, the system was inverted to allow the biotin to contact the substrate surface. The biotin was given 30 minutes to react and bond with the functionalized substrate surface before the stamp was removed and the substrate surface was washed using ethanol. The substrate was submerged in a solution containing $0.1 \mu \mathrm{M}$ of unlabeled streptavidin and allowed to incubate for 30 minutes. The substrate was washed repeatedly in a buffer solution and pattern generation was confirmed using a confocal fluorescence microscope.

Soft lithography offers a method of forming patterns of biological materials with feature sizes below $1 \mu \mathrm{m}$. This patterning method can be applied to flat and non-flat substrates due to the high level of conformity of the PDMS stamp. Soft lithography could be used in conjunction with any of the deposition methods discussed in the previous section, making it a very versatile patterning technique. Soft lithography does require expensive and specialized equipment if the process is to be performed using a photoaligner. If no aligner were used, it would be difficult to accurately align the PDMS stamp with any pattern already present on the substrate. Because the goal of this research centers on the integration of PM films with pre-patterned microelectronic substrates, a soft lithographic aligner system would be required. Due to the lack of availability of a soft lithographic aligner system, this patterning method was not pursued as part of this research project. 


\subsubsection{Electrodeposition Based Patterning}

The most commonly used technique for patterning PM films involves patterning a conductive substrate prior to the deposition of the PM film. As was discussed previously, the electrodeposition process requires both top and bottom electrodes to create an oriented PM film that is bound to the substrate. It is possible to selectively deposit oriented PM films using this deposition method if the substrate is patterned accordingly. When performing electrodeposition, oriented PM films will only be deposited on the conductive areas of the positive electrode connected to the circuit causing the electric field. Thus, if patterned conductive areas of a substrate are used as the positive electrode in the electrodeposition circuit, a patterned oriented PM film will be deposited.

A PM film was successfully integrated into a microelectronic device by Battacharya et al. [28] by electrodepositing PM onto the gate pad of a modulation-doped field effect transistor (MODFET). The MODFET architecture was fabricated using standard metalization, photolithography liftoff and doping techniques. The MODFET Schottky gate contact was formed by selective area deposition of $\mathrm{Ti} / \mathrm{Pt} / \mathrm{Au}$ to form an electrode pad that acted as the anode in the subsequent electrodeposition. A $10 \mu \mathrm{l}$ drop of PM fragments suspended in water, having an optical density of $20 / \mathrm{cm}$, was placed on top of the electrode pad and the electrodeposition process was performed. A platinum plate was used as the cathode and was separated from the anode by a $2 \mathrm{~mm}$ gap. A current of $\sim 2$ $\mathrm{mA}$ was applied for approximately 3 minutes to perform the electrodeposition. The PM was allowed to dry, and an ITO top electrode was placed over the PM film. The PM was exposed to $594.1 \mathrm{~nm}$ laser light at an intensity of $0.7 \mathrm{~mW} / \mathrm{mm}^{2}$. The maximum photocurrent resulting from the PM being exposed to the laser light was $2.7 \mathrm{~mA}$. Photocurrent spikes were observed when the light source was turned on as well as when turned off. This detector platform could only detect a change in the lighting conditions, and thus could be useful for motion and edge detection. 
Takei et al. [29] presented a method in which a simple photodetector was fabricated using the electrodeposition method. Two tin oxide coated glass plates served as substrates. A suspension of PM was placed between the two conductive plates and a voltage was applied that electrodeposited the PM onto the anode. The PM film was allowed to dry at a humidity of $80 \%$ to prevent cracking of the film. This process was followed again to obtain a second PM film deposited onto tin oxide. A section of the film deposited on each slide was removed in such a way that the area removed from one film exactly matched the area remaining on the other film. The two slides were then brought together so that the remaining PM film made contact with the area where the film was removed from the opposite slide. This assembly resulted in a dried PM film between two conductive substrates with different regions of the film oriented opposite from each other. It was found that by measuring the photoresponse of the PM films, the detection of the zero crossing of a light source across this simple photodetector was possible. This result demonstrated the feasibility of creating a PM based photodetector capable of optical edge detection without the aid of external circuitry.

The main advantage of the electrodeposition based patterning technique is its simplicity. All that is required to pattern PM films using this method is a patterned conductive substrate that can be electrically accessed. Thick layers of oriented PM can be deposited and oriented quickly. These thick films have proven advantageous for a wide variety of studies performed to better understand and characterize the optioelectrical properties of PM films.

The disadvantage of this patterning technique is its lack of versatility. In order to utilize this patterning technique, electrodeposition is the only PM film deposition method that can be applied. While electrodeposition is the most widely used PM deposition technique described in the literature, it has proven difficult to control the number of PM layers deposited. For studies and applications that require a known number of PM layers, a different patterning technique is required. A second limitation of this patterning 
technique is that the conductive portions of the substrate used as the anode must be electrically accessible. This becomes difficult when PM films are to be integrated into a more complex microelectronic system.

\subsubsection{Laser Ablation}

Laser ablation is a process in which a laser source is used to remove solid material from a substrate. Energy from the laser beam is absorbed by the surface of the substrate, causing some of the substrate to be vaporized. The advantage of laser ablation over other types of laser cutting is that material is not removed through heat, but through absorbing the laser energy, which in turn disrupts the molecular bonds of the substrate. This allows material to be removed with very little localized heating around the machining site.

Haronian and Lewis [30] developed a PM film patterning technique based on laser ablation. First, a PM film was deposited onto a tin oxide coated quartz substrate by electrodeposition to form an oriented film. An electron microscope grid having parallel bars $30 \mu \mathrm{m}$ thick and $30 \mu \mathrm{m}$ apart was used as a mask, and was placed on top of the film. A Lambda Physik model 103 argon fluoride excimer laser operating at $193 \mathrm{~nm}$ was used to ablate the PM film exposed between the bars on the mask. During ablation, the entire system was cooled to $10^{\circ} \mathrm{C}$ in order to reduce the heating of the remaining PM film. The grid was removed and a gold film was evaporated over the entire sample. The grid was placed back on the sample in a perpendicular orientation to its initial position, and the sample was ablated by the laser. The process resulted in $30 \mu \mathrm{m}$ x $30 \mu \mathrm{m}$ square pixels of PM coated with gold. A second sample measuring $50 \mu \mathrm{m}$ x $800 \mu \mathrm{m}$ was fabricated in the same manner, and the photoresponse of the remaining PM film was confirmed.

The work presented by Haronian and Lewis was the only attempt at patterning PM films using laser ablation found in the literature. Because many of the details of the 
patterning process were not discussed, it is unclear how viable this method would be for creating large area patterns of PM films. Possible issues with this process include the time required to pattern a large area, damaging the remaining PM film, and damaging the underlying substrate. While laser ablation of PM films is an interesting patterning technique that has been demonstrated to be successful, many questions about the overall effectiveness of this method remain. 


\subsection{Preparation of Purple Membrane Suspensions}

The growth of $H$. salinarum and subsequent extraction and purification of purple membrane was of vital importance to the work described herein. In order to develop a patterning technique capable of high resolution pattern generation without negatively impacting the functionality of the PM film, it was critical to start with a well characterized and highly purified liquid PM suspension.

\section{$2.1 \quad$ Growth of $H$. salinarum}

As was stated previously, the bR molecule is found in the purple membrane of the bacterium $H$. salinarum. To obtain a quantity of PM suitable to sustain research efforts, the decision was made to locally grow $H$. salinarum and harvest the PM. This decision was based on the fact that commercially available preparations of this protein are expensive, with an uncertain purity.

To begin the growth process, the S9-P strain of Halobacterium salinarum was obtained from John Spudich at the University of Texas Medical School. This strain was designed to overproduce PM in an environment having a high concentration of dissolved oxygen. Whereas the wild strain of $H$. salinarum requires light in order to produce PM, the S9-P strain did not require the presence of light. Two $1 \mathrm{~mL}$ vials of the strain were obtained on October 13, 2004. After inoculating the initial cultures, the two source strain vials containing the remaining $H$. salinarum were placed in cold storage, one at $4^{\circ} \mathrm{C}$ and one at $-70^{\circ} \mathrm{C}$.

To grow the bacteria, a growth protocol developed by J. A. Stuart et al. was followed. The cell growth took place in a liquid media broth known as the basal salts medium. The addition of bacteriological peptone, obtained from Oxoid, to the basal salts medium 
supplied the nutrient source for the bacteria. Table 1 below shows the reagents and concentrations required for the preparation of this medium. The medium was typically made in $4 \mathrm{~L}$ batches. Two $5 \mathrm{~L}$ Erlenmeyer flasks were used to hold $2 \mathrm{~L}$ of total medium each. Each flask was filled with $1.5 \mathrm{~L}$ of ddi water. Magnetic stir bars were placed in each flask, and the flasks were placed on stir plates to provide agitation.

Table 1: Reagents and concentrations required to prepare $H$. salinarum growth medium.

\begin{tabular}{|l|c|c|c|}
\hline Reagent & Concentration & Per 1 Liter & Per 10 Liters \\
\hline $\mathrm{NaCl}$ & $4.28 \mathrm{M}$ & $250 \mathrm{~g}$ & $2.5 \mathrm{~kg}$ \\
\hline $\mathrm{MgSO} 4$ (anhydrous) & $81.1 \mathrm{mM}$ & $9.77 \mathrm{~g}$ & $97.7 \mathrm{~g}$ \\
\hline $\mathrm{KCl}$ & $26.8 \mathrm{mM}$ & $2 \mathrm{~g}$ & $20 \mathrm{~g}$ \\
\hline $\mathrm{NH} 4 \mathrm{Cl}$ & $93.5 \mathrm{mM}$ & $5 \mathrm{~g}$ & $50 \mathrm{~g}$ \\
\hline Sodium Citrate. 2H2O & $10.2 \mathrm{mM}$ & $3 \mathrm{~g}$ & $30 \mathrm{~g}$ \\
\hline Glycerol & $137 \mathrm{mM}$ & $1 \mathrm{ml}$ & $10 \mathrm{ml}$ \\
\hline KH2PO4 & $0.735 \mathrm{mM}$ & $0.1 \mathrm{~g}$ & $1 \mathrm{~g}$ \\
\hline CaCl2 (anhydrous) & $1.4 \mathrm{mM}$ & $0.2 \mathrm{~g}$ & $2 \mathrm{~g}$ \\
\hline Bacteriological Peptone & - & $10 \mathrm{~g}$ & $100 \mathrm{~g}$ \\
\hline
\end{tabular}

The reagents listed in Table 1 were then added to the water in the order they appear and allowed to mix for approximately 30 minutes, until the chemicals were fully dissolved. The $\mathrm{pH}$ of each solution was raised to 7.2 with $5 \mathrm{M} \mathrm{NaOH}$. After the $\mathrm{pH}$ was corrected, ddi water was added to bring the total volume of each flask up to 2L. The top of each flask was covered with aluminum foil, and the flasks were autoclaved (15 psi, $121^{\circ} \mathrm{C}, 20 \mathrm{~min}$ ) to ensure the sterility of the media broth. The medium was placed in a refrigerator at $4^{\circ} \mathrm{C}$ immediately following autoclaving, and stored until needed.

The initial growth study involved growing $H$. salinarum both in a liquid medium as described above, as well as on agar plates. Growing bacteria colonies on plates allowed for visual confirmation of growth. H. salinarum colonies are known to appear red or purple when grown on plates due to the presence of retinal in the bR. Prior to autoclaving the first batch of medium, Bacto-agar obtained from Difco was added to a small amount of the medium in a separate flask to make solid agar plates for growth. 
After the liquid medium was autoclaved, the liquid was allowed to cool. The smaller flask of medium containing agar was poured onto Petri plates and allowed to cool and solidify. Two agar plates were inoculated with the $H$. salinarum strain by dipping an inoculating loop in the source strain and dragging the loop across the agar plates. The plates were placed in an incubator at $40^{\circ} \mathrm{C}$. Red colonies were visible on the plates after five days of growth. The plates were allowed to grow for two weeks in the incubator. The plates were then placed in a refrigerator at $4^{\circ} \mathrm{C}$ to stop further growth of the bacteria.

Immediately after inoculation of the agar plates, $10 \mathrm{~mL}$ of the liquid medium was inoculated with $10 \mu \mathrm{l}$ of the source strain in a $125 \mathrm{~mL}$ sidearm flask. The sidearm flask was placed in a New Brunswick Scientific gyrotory water bath shaker with a water temperature of $40^{\circ} \mathrm{C}$ and a high amount of agitation (setting of 5 to 8 on bath shaker). The high agitation was provided in order to aerate the bacteria, which dramatically influenced its growth rate. Immediately after inoculation, the liquid culture had a clear yellow appearance. After 24 hours, the liquid culture became visibly turbid indicating that the bacteria were growing. The liquid culture was maintained by transferring $200 \mu \mathrm{l}$ of inoculated liquid culture into $10 \mathrm{~mL}$ of sterile medium each time the culture in the sidearm flask surpassed its logarithmic growth phase. This was done in order to provide the bacteria with an abundant nutrient source, and to keep healthy cells growing in the liquid culture.

To better understand the growth of the bacteria, its growth was charted. Measurements were taken every five hours using a Klett Summerson photoelectric colorimeter. This instrument gave a fast measurement in "Klett units", which provided a relative measurement of the optical density of the liquid. The optical density was then related to the cell density of the bacterial growth in the liquid medium. Measurements were taken by allowing the liquid culture to drain into the sidearm portion of the sidearm flask. The sidearm was placed in the photoelectric colorimeter. Before each 
measurement was taken, the instrument was calibrated using sterile $H$. salinarum growth medium in a second sidearm flask.

A total of 13 measurements were taken, and the measurements were plotted on a semi-log graph shown in Figure 2. Optimally, the bacteria cultures should be kept growing in the logarithmic growth stage, which is seen as a linear trend on a semi-log graph. By charting these data points, an approximate optical density where the bacteria growth falls out of logarithmic growth was obtained. The optical density value was found to be approximately "50 Klett units” for this specific experimental setup.

\section{Optical Density of Liquid Culture}

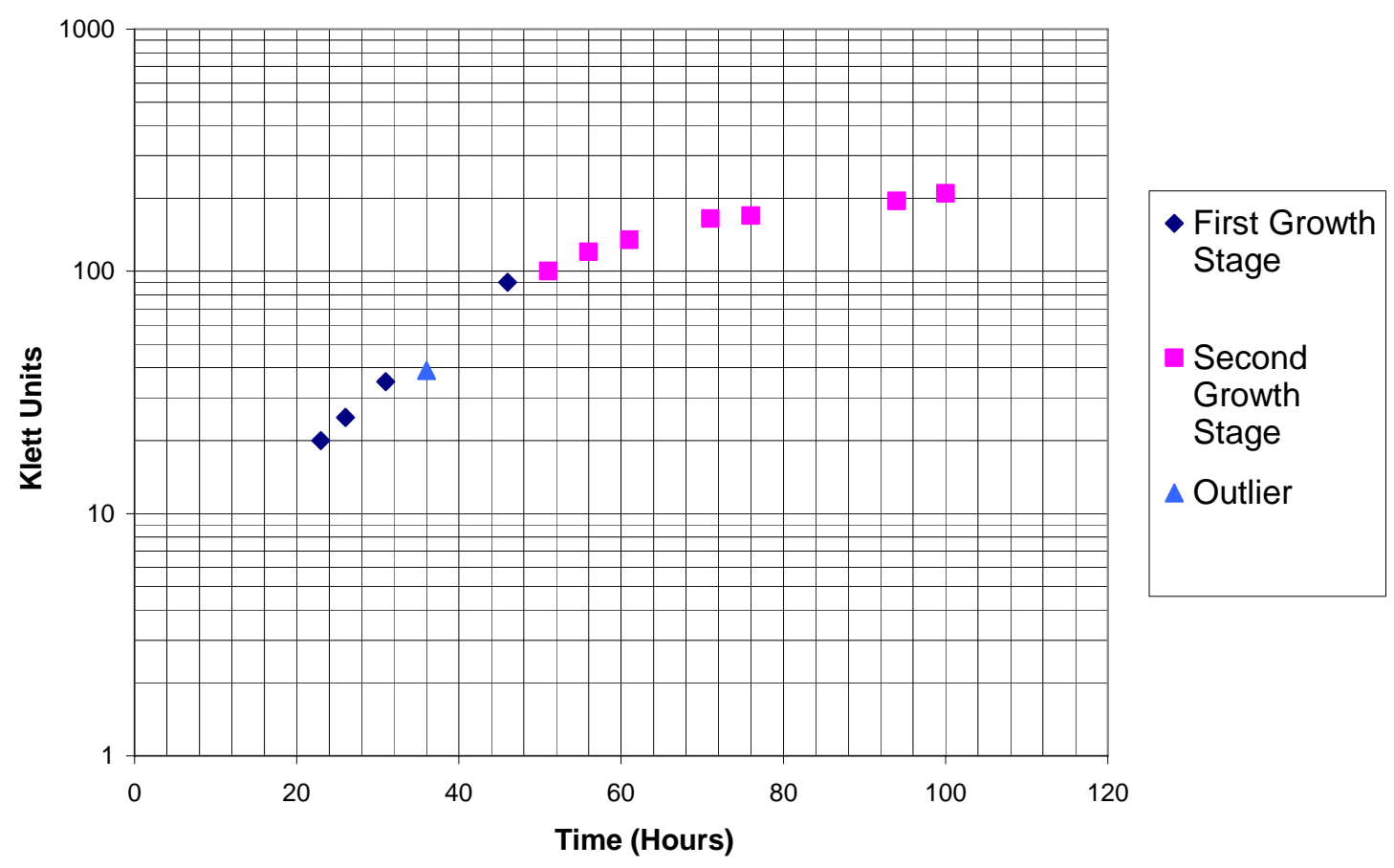

Figure 2: H. salinarum growth in a sidearm flask placed in a water bath of 40 degrees Celsius measured on a Klett Summerson Photoelectric Colorimeter.

Figure 2 was also useful in that it provided the regeneration time of the bacteria in the liquid medium. This regeneration time is the amount of time it takes the density of the 
culture to double while the bacteria are in a logarithmic growth phase. The regeneration time for this experimental setup was approximately ten hours.

After a basic understanding was obtained of how this bacteria grew under uncontrolled conditions, a quantity of liquid culture was grown to extract an appreciable amount of purple membrane. The paper by Stuart et al. stated that the best way to obtain high PM yields when growing the S9-P strain of $H$. salinarum was to use a fermentor. Because of this, initial large scale growth was done using the New Brunswick Scientific Bioflo III Continuous/Batch Fermentor as shown in Figure 3. This fermentor allowed for the continuous growth of the bacteria as well as the ability to monitor and adjust agitation, aeration, $\mathrm{pH}$ level, and temperature. One liter of medium was innoculated with $150 \mathrm{~mL}$ of liquid culture that had been growing in shaker water bath in a $250 \mathrm{~mL}$ sidearm flask. The $\mathrm{pH}$ level of the inoculated medium was maintained at 7.2 by peristaltic pumps transferring $5 \mathrm{M} \mathrm{HCl}$ acid or $5 \mathrm{M} \mathrm{NaOH}$ base into the medium as necessary. The temperature was maintained at $40^{\circ} \mathrm{C}$. The aeration and agitation settings were varied during the growth of the initial $10 \mathrm{~L}$ quantity in an attempt to optimize the growth rate of the bacteria. 


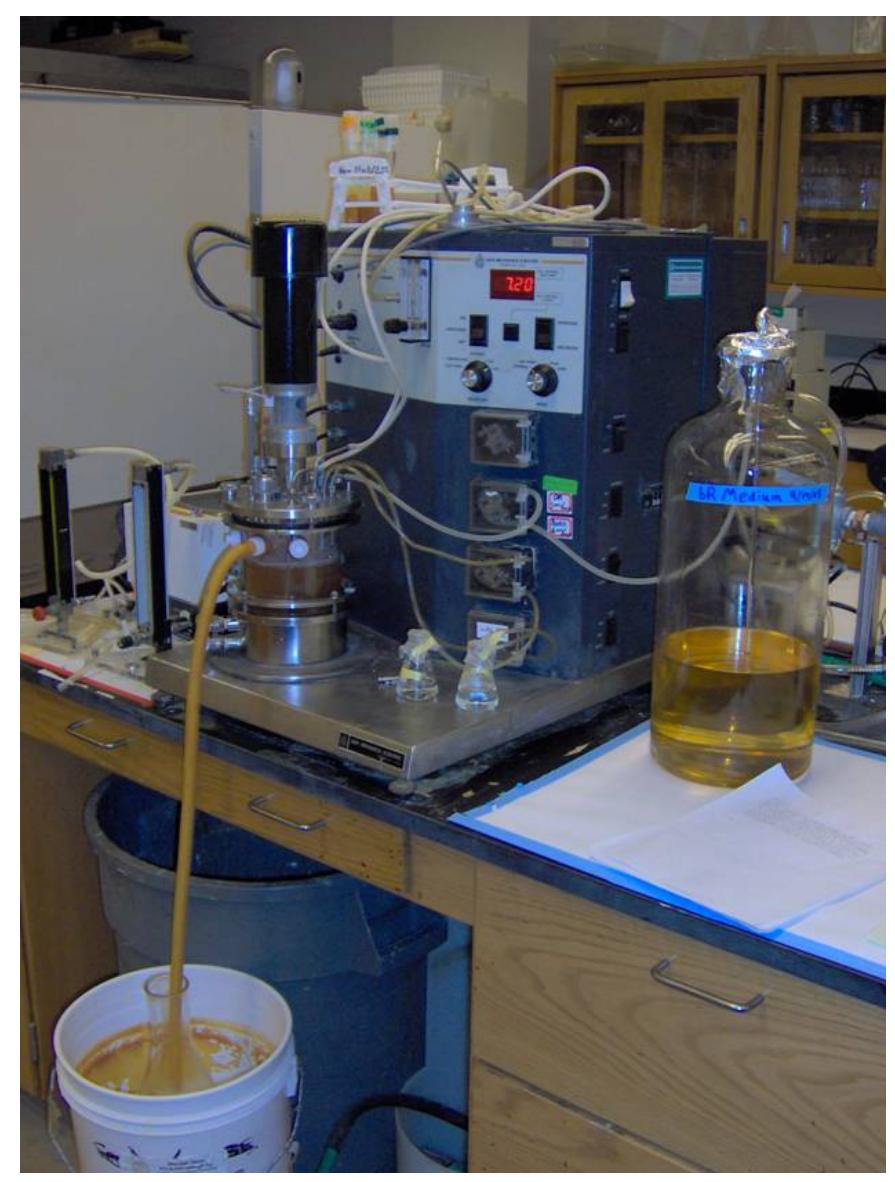

Figure 3: New Brunswick Scientific Bioflo III Continuous/Batch Fermentor growing H. salinarum.

The medium was allowed to grow in a closed loop system until the medium reached a desired cell density. The cell density of the inoculum was measured every two hours using both the Klett Summerson system as well as a Beckman Model DU 640 spectrophotometer. The spectrophotometer gave absorbance values for the culture, which could then be related back to the cell density of the liquid. The initial growth curve for the Bioflo is shown in Figure 4. The abrupt drop in the cell density at 20 hours was due to an equipment failure that required transferring the inoculated liquid to another reactor vessel. 


\section{Bioflo Growth Curve}

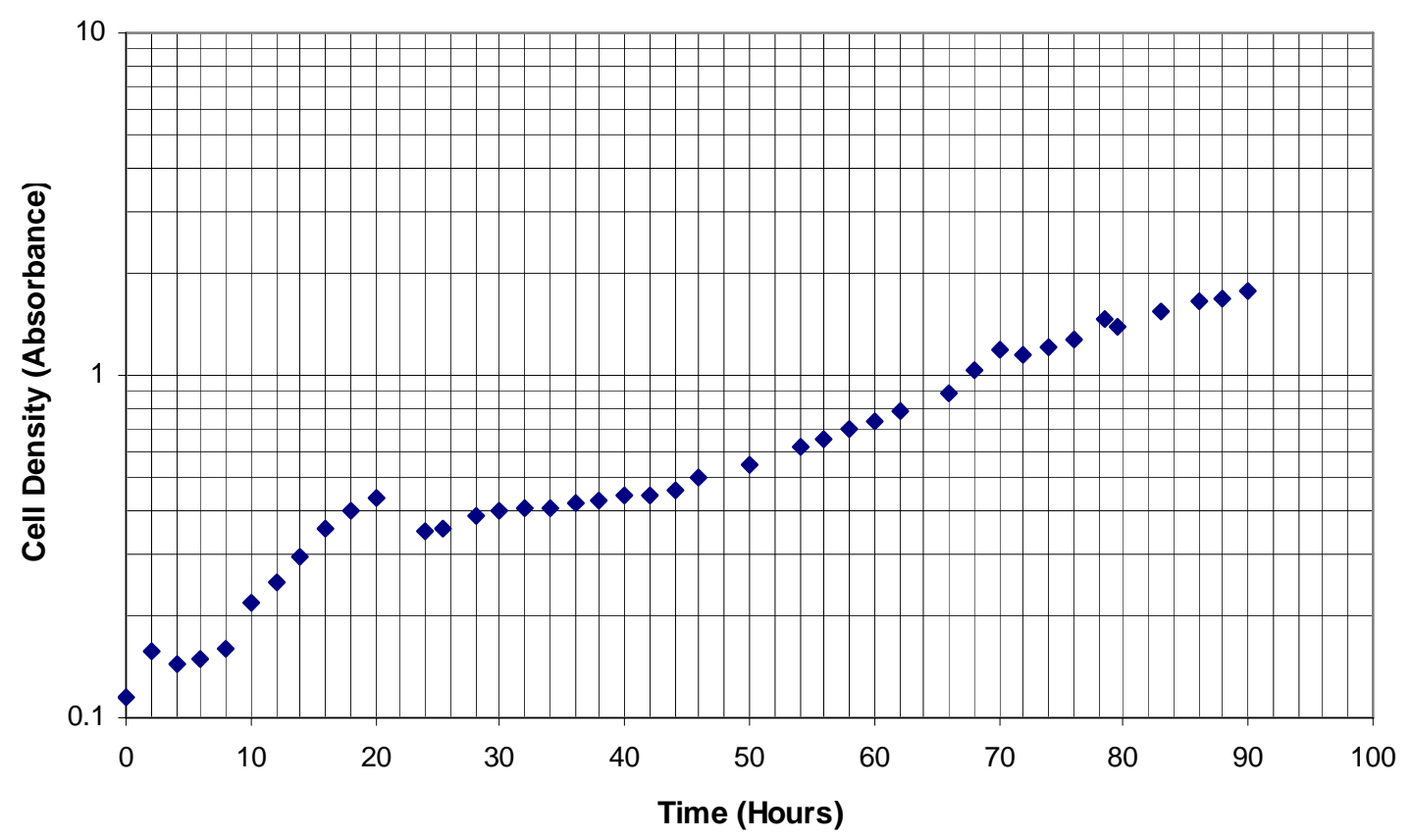

Figure 4: $H$. salinarum growth versus time grown in the New Brunswick Scientific Bioflo III Continuous/Batch Fermentor. Absorbance measurements were taken with the Beckman Model DU 640 Spectrophotometer.

Several issues became quickly apparent with the continuous fermentation growth method described above. Both growth rates and PM yields using the batch fermentation method were much lower than expected. It proved to be extremely difficult to predict the growth rate of the bacteria in the reactor. Because of this instability in the growth rate, it was impossible to maintain a constant cell density in the reactor vessel. Salt crystals were also found in the bottom of the reactor vessel when the system was shut down. It was hypothesized that due to the elevated temperature in the reactor and the fact that the system was not sealed, some water was evaporating out of the reactor. This loss in water content in the media broth would cause some of the salt to precipitate out of suspension and collect on the bottom of the reactor due to the extremely high salinity of the medium. 
The continuous growth method also required constant monitoring to test the cell density and ensure that no major changes had taken place. Because the growth was so unpredictable, cell density measurements needed to be taken multiple times every day to ensure that the proper cell density inside the reactor was being maintained. A new method of growth was required that would be more predictable and require less frequent monitoring if large quantities of PM were to be produced.

It was observed early in the growth of $H$. salinarum that the bacteria grew quickly and predictably in small sidearm flasks placed in a temperature regulated shaker and agitated at a rapid rate. In fact, the growth rates observed in the sidearm flasks were higher than the best growth rates observed using the Bioflo fermentor. Based on this observation, a large batch style growth protocol was developed. This protocol made use of the same growth medium as described earlier in this section. The medium was placed in four $2800 \mathrm{~mL}$ Pyrex Fernbach flasks, with each flask holding $1 \mathrm{~L}$ of medium. The medium was then inoculated with $100 \mathrm{~mL}$ of growth medium containing H. salinarum. The four flasks were sealed using rubber stoppers and placed in a Lab-Line IncubatorShaker. The temperature was set at $40^{\circ} \mathrm{C}$ and the agitation was set at $100 \mathrm{rpm}$. A $75 \mathrm{~W}$ incandescent light source was placed above the flasks to provide light illumination during the growth of the bacteria. The bacteria were allowed to grow in the flasks for 5-7 days, until an optimal cell density was reached.

This simplified growth protocol has proven to be very effective at growing large amounts of $H$. salinarum in a short amount of time. By moving forward with the purification process discussed in the next section, large yields of PM have been harvested from the cells grown with this method. This growth method has also solved the problem of large amounts of time being required to monitor the cell growth. Because nothing is added or subtracted from the flasks during growth, the only question is when to remove the cells from the flasks and inoculate a new batch of cells. The process of harvesting the cells and inoculating a fresh batch of medium requires approximately three hours of time 
per week to accomplish, a vast improvement over the time requirements to keep a continuous growth in operation.

\subsection{Purification of Purple Membrane}

After a liquid culture was harvested, the purple membrane was isolated through a series of purification steps. A basic description of the steps required to obtain purified PM is shown in Figure 5. The liquid culture was first poured into six Beckman JA-14 type centrifuge bottles. Each bottle holds approximately $100 \mathrm{~mL}$ of culture. The centrifuge bottles were then spun in a Beckman J2-21M/E centrifuge using a Beckman J14 type rotor for fifteen minutes at $8000 \mathrm{rpm}$. The centrifuge temperature was $4^{\circ} \mathrm{C}$, and the cells were kept on ice or in a refrigerator for the remainder of the purification process. After the tubes were spun, dark red pellets formed at the bottom corner of the tubes. This pellet contained whole $H$. salinarum cells. The supernatant was discarded and more harvested culture was added to the same two tubes. The above centrifuge process was repeated using the same six tubes until solid pellets of the desired size were achieved. Typically, a total of 20L of cell culture was spun in the centrifuge and collected in the same six bottles before the next step in the purification process was performed. This was done to save time, as the purification process was time consuming. 


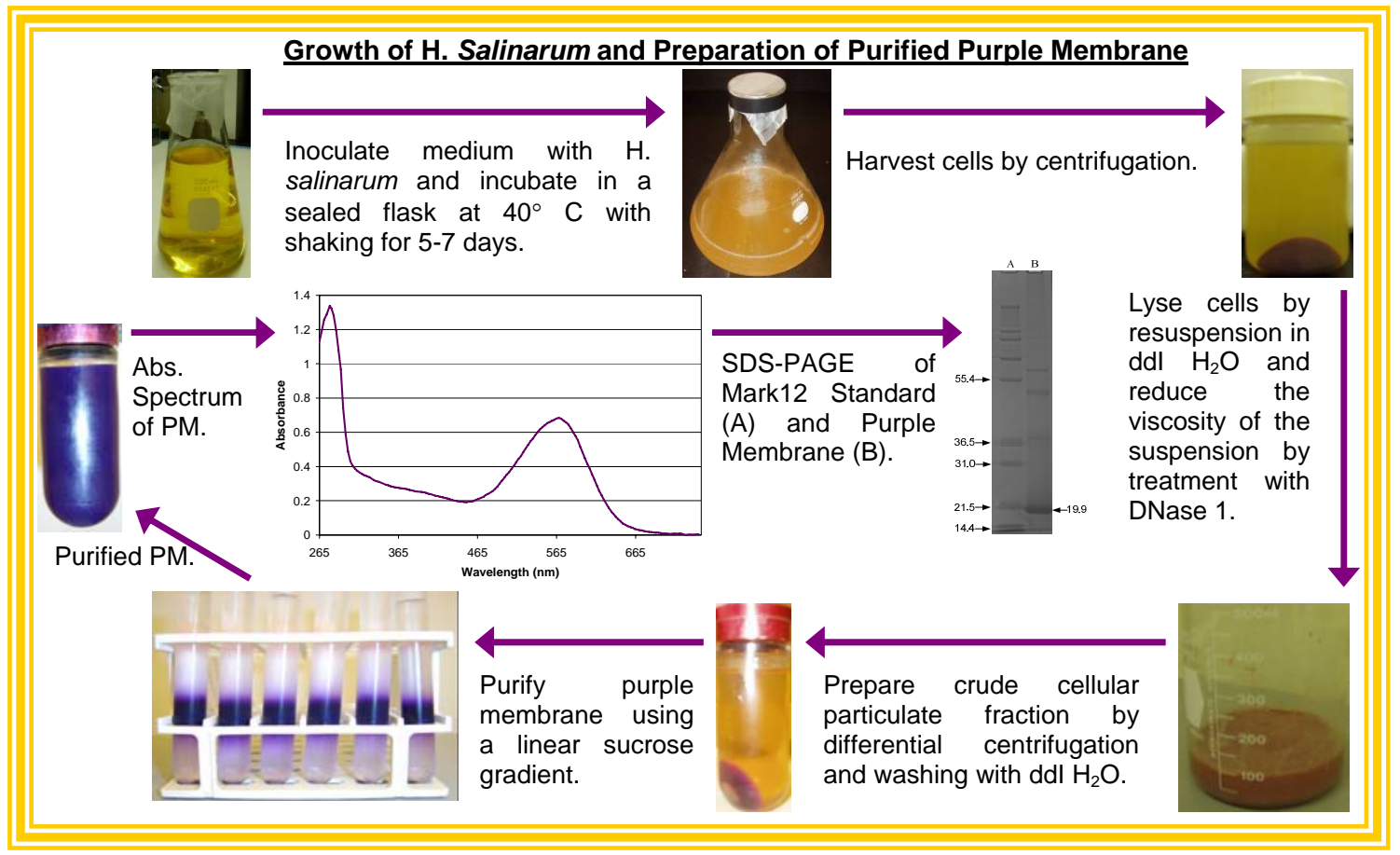

Figure 5: Flow chart describing the basic steps taken to grow and purify PM

After the pellets were formed and all of the supernatant was drained off, the pellets were removed from the centrifuge tubes and placed in a $950 \mathrm{~mL}$ glass beaker. The pellets were then resuspended in a small amount of ddi water and mixed to form a thick red liquid. The water osmotically lyses the cells, breaking them apart, and allowing the DNA chains to unravel. Because of this uncoiled DNA, the suspension became increasingly viscous. DNase I (Bovine) was added in a ratio of 1 unit per $\mathrm{mL}$ to the cell suspension in order to break down the DNA chains. The suspension was then placed on a magnetic stir plate and stirred for 24 hours at room temperature to allow the DNase to reduce the viscosity of the cell suspension.

After the cell suspension viscosity was reduced to approximately that of water, the suspension was poured into Beckman JA-20 centrifuge bottles. The bottles were centrifuged in a Beckman XL-90 Ultracentrifuge using a Beckman JA-20 rotor for fifteen minutes at $5000 \mathrm{rpm}$. This caused the large cell debris to collect in the bottom of the 
centrifuge tubes, forming a pellet. After the centrifugation was complete, the supernatant was saved and the pellets were discarded. The remaining supernatant contained the desired PM along with smaller sized cell debris.

The supernatant was again poured into Beckman JA-20 centrifuge tubes and spun using the Beckman JA-20 rotor for 30 minutes at 35,000 rpm. The pellets that formed in the bottom of the centrifuge tubes were kept and the supernatant was discarded. The pellets had a purple to red color while the supernatant appeared yellow. This process was then repeated by resuspending the pellets in ddi water and running the centrifuge with the same rotor and settings as described above, until the supernatant being discarded was clear. This step washed the purple membrane of small cell debris as well as any remaining components of the liquid medium in which the cells were grown. After the supernatant was observed to be clear, it was poured off and the pellets were combined into a single centrifuge tube and resuspended in 2 - $4 \mathrm{~mL}$ of ddi water. The result was a royal-purple liquid.

The last purification step involved passing the purple membrane suspension through a linear sucrose gradient to separate the purified purple membrane from other components still present in the suspension. Two densities of sucrose were prepared, one at $20 \%$ sucrose-to-water and the other at $46 \%$ sucrose-to-water. The sucrose solutions were prepared by mixing reagent grade sucrose with ddi water and allowing the sucrose to be suspended in the water. The percentage of sucrose present in the solution was confirmed using a Baltimore Instruments Abbe Refractometer and adjusted to the desired densities. A Hoefer gradient former was used to create a linear sucrose gradient in each of six Beckman SW41Ti centrifuge tubes. The purple suspension was layered on top of the linear gradient and centrifuged using the Beckman SW41Ti swinging bucket rotor at 20,000 rpm for 18 hours. The linear sucrose gradients separated the various components left in the purple suspension into discrete color bands, as in Figure 6. The purple band 
was removed from the gradient using a Pasteur pipette. This purple band consisted of pure purple membrane suspended in sucrose.

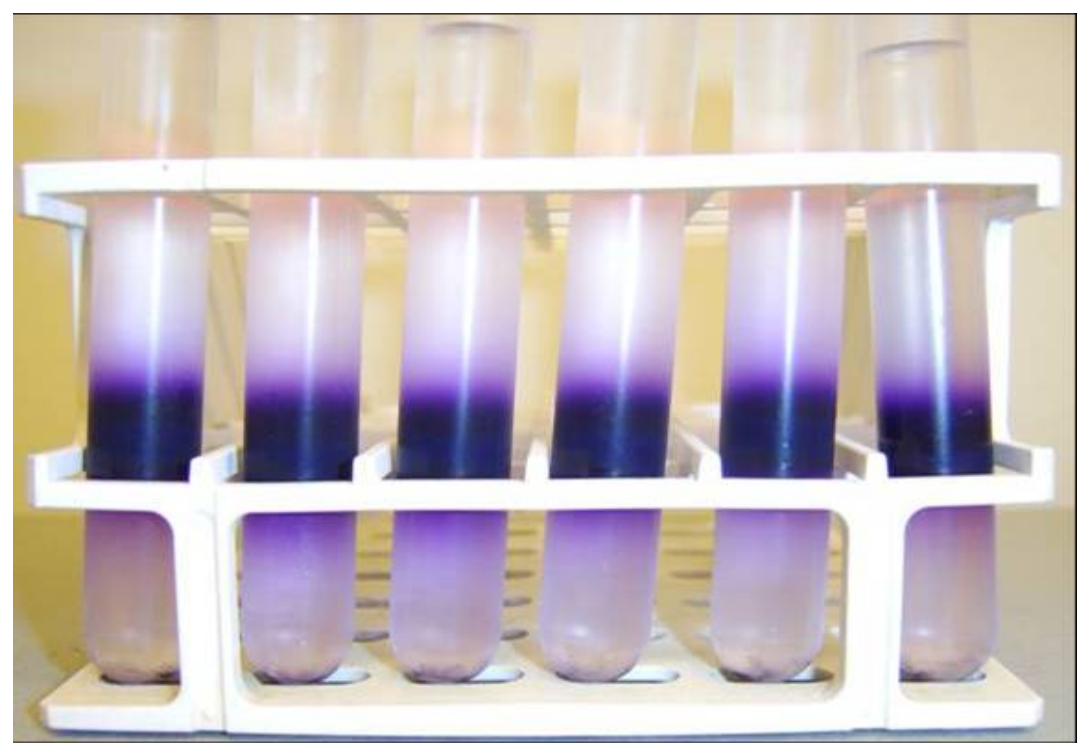

Figure 6: The result of centrifuging PM through linear sucrose gradients.

The PM removed from the gradients was placed in a single JA-20 centrifuge bottle and ddi water was added to dilute the concentration of sucrose in the solution. The PMsucrose suspension must be diluted at a ratio larger that 1:1 with ddi water in order for the PM to properly pellet in the bottom of the centrifuge bottle. The PM was then spun at 37,500 rpm for one hour using the Beckman JA-20 rotor. The supernatant was poured off and the pellet resuspended in a 1-3 mL of ddi water. This dark purple liquid contained ultra-pure purple membrane. The liquid was left in the centrifuge bottle and the top of the bottle was sealed to keep the liquid from evaporating. This suspension was then placed in a refrigerator at $4^{\circ} \mathrm{C}$. 


\subsection{Characterization of Purified Purple Membrane}

The purity of each PM preparation was evaluated by sodium dodecyl sulfate polyacrylamide gel electrophoresis (SDS-PAGE). The gel used was an Invitrogen 12\% Bis-Tris gel, and the procedure was performed in an Invitrogen XCell SureLock MiniCell. A standard SDS-PAGE protocol provided with the Invitrogen XCell SureLock Mini-Cell was followed to perform the analysis. A typical result of an SDS-PAGE analysis is shown in Figure 7. This analysis gives the apparent molecular weight (Mr) of the material being analyzed. The coomassie blue stained bands present in each lane indicate the molecular weights of the various components within that lane. In addition to a solution containing PM, a Mark-12 standard was run along side the PM. The Mark-12 standard contains a series of proteins with known molecular weights. This standards lane then serves as a reference used to approximate the molecular weight of the components present in the PM solution. The molecular weight of pure PM is well documented as 26,000 Da in the literature [31].

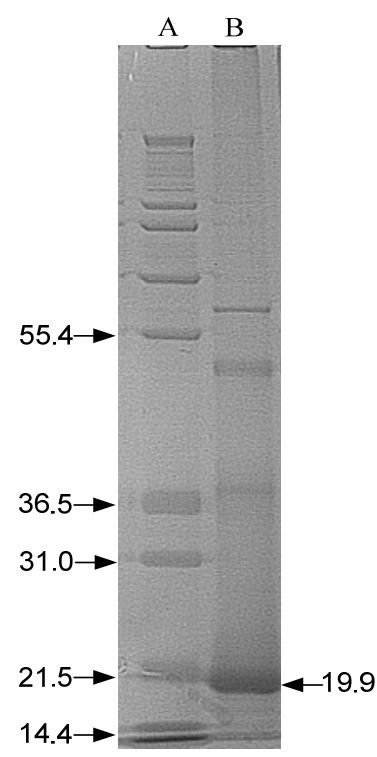

Figure 7: SDS Gel with column A containing a Mark-12 standard (left) and column B (right) containing a PM solution. 
As can be seen from the resulting SDS-PAGE analysis, the molecular weight of the major band in this purification was considerably lower $(\sim 20 \mathrm{kDa})$ than the expected result of $26 \mathrm{kDa}$. However, as reported by Bridgen and Walker [32], the apparent molecular weight of PM as determined by SDS-PAGE was found to be $20 \mathrm{KDa}$. This value is in good agreement with all of the SDS-PAGE analyses performed on the PM preparations. The reason for the lower molecular weight result found by the SDS-PAGE analysis is most likely due to how PM binds to SDS during the analysis. These SDS-PAGE results provide an indication that the revised growth process, as well as the purification process, result in a PM suspension with a purity exceeding 90\%.

A second method of verifying the presence of PM in a suspension is to analyze its optical absorption spectra. PM has two distinct absorption peaks at $280 \mathrm{~nm}$ and $570 \mathrm{~nm}$. The ratio of the absorption maxima of PM at $280 \mathrm{~nm}$ and $570 \mathrm{~nm}$ is approximately 2:1. If the PM in a suspension is damaged, this absorption intensity ratio will no longer be observed. This analysis method does not provide any indication of the overall purity of the suspension, but rather the condition of the PM present in the suspension. All absorption spectra measurements were taken using a Nanodrop ND-1000 spectrophotometer in the UV-Vis mode. 


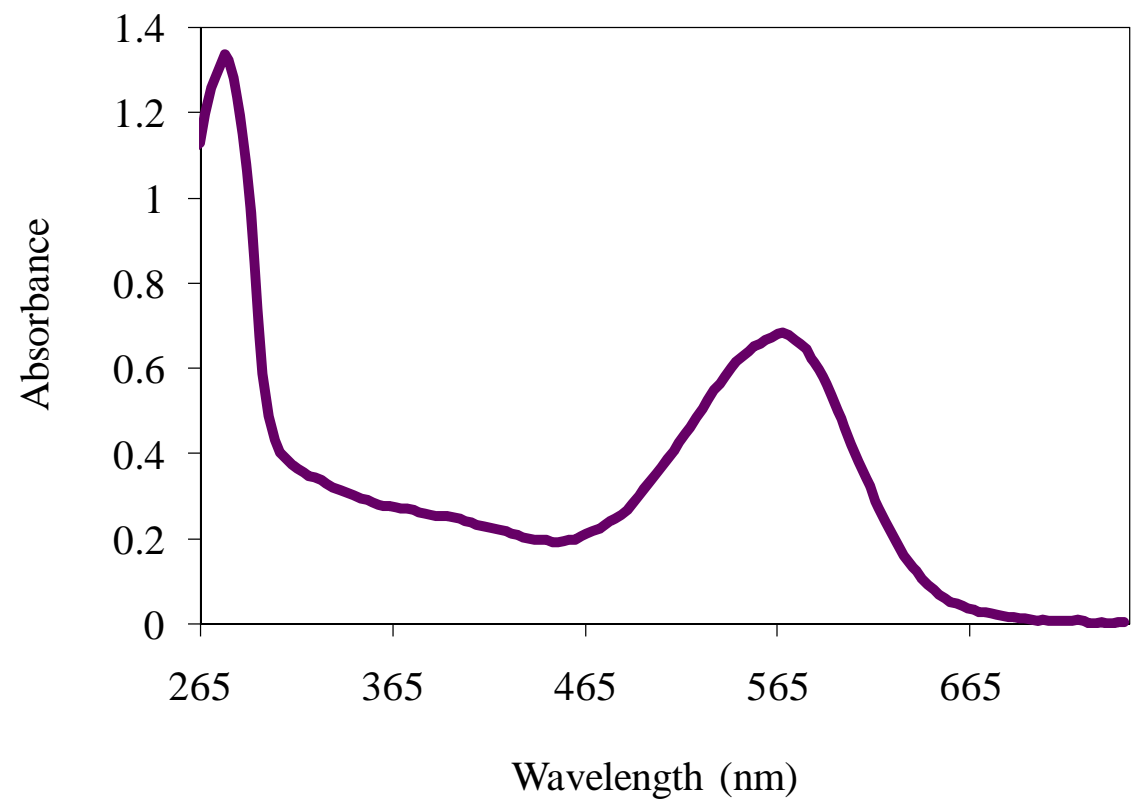

Figure 8: Absorbance spectra of a purified PM liquid suspension taken by a Nanodrop ND-1000 spectrophotometer.

The concentration of PM contained in a liquid suspension was calculated using the Beer's Law:

Equation 1: $A=\varepsilon * c * 1$

A is the absorbance of the sample measured using a Nanodrop ND-1000 spectrophotometer. $\varepsilon$ is the extinction coefficient of bacteriorhodopsin; $63,000 \mathrm{~mol}^{-1} \mathrm{~cm}^{-}$

1 . $\mathrm{c}$ is the concentration of bacteriorhodopsin in the suspension and 1 is the optical path length of the measurement. This calculation gave the concentration of PM in a liquid suspension in $\mu \mathrm{mol} / \mathrm{ml}$. This value is then multiplied by the molecular weight of PM (26000 $\mu \mathrm{g} / \mu \mathrm{mol}$ ), giving PM concentration in $\mu \mathrm{g} / \mathrm{ml}$. This calculation was used to evaluate the total weight of PM produced by each PM preparation. After the evaluation 
of the PM suspension was complete, the PM suspension was aliquoted into small volumes $(50-200 \mu \mathrm{l})$ and stored at $-80^{\circ} \mathrm{C}$ until needed. 


\subsection{Focused Ion Beam Patterning of Dried PM Films}

Focused Ion Beam (FIB) machining was investigated during the early stages of this work as a possible method of patterning PM films. FIB machining uses a beam of gallium ions focused on the surface of a substrate. As the gallium ions collide with the atoms comprising the substrate, some of the substrate atoms are dislodged. This removes atoms from the surface of the substrate in the region where the gallium beam is focused, while some portion of the incident gallium ions are implanted.

All of the FIB work presented below was performed using a Hitachi FB-2000A Focused Ion Beam system. The FIB was equipped with both an onboard vector scan controller and a high resolution Nabity scanning system to control the patterns produced by the gallium beam.

Unlike some of the other patterning methods discussed in Section 1.4, FIB machining does not control where the PM film is deposited. A liquid suspension of PM in ddi water was first deposited onto a substrate and allowed to dry. The FIB was then used to selectively remove the PM film from the substrate. As with any type of electron ion optics device, the FIB is susceptible to the effects of charging of the specimen surface. Charging occurs when a specimen is not electrically grounded to the specimen holder. This lack of grounding can be caused by improperly installing the specimen into the holder, or by working with a non-conductive specimen. Electrical charge builds up on the surface of the sample and is periodically discharged into the specimen chamber. The effects of charging range from poor quality images to the destruction of some portions of the sample being viewed. Biological samples typically have low electrical conductivity, and are often a challenge to view using electron ion optics without a conductive coating. This is particularly true for PM, which has a resistivity on the order of G $\Omega$-cm. In order 
to reduce the effects of charging, PM films were formed on conductive substrates including silicon wafers coated with aluminum or gold, as well as brass substrates.

\subsection{Physical Characteristics of FIB Machined Dried PM Films}

The first set of studies performed using the FIB addressed the physical characteristics of dried PM films that had been machined using the FIB. Because no other FIB work on PM films has been reported, the physical effects of the gallium beam on the PM film were unknown. In the first study, a piece of silicon wafer coated with an aluminum film of unknown thickness was used for the substrate. The aluminized silicon wafer provided a conductive substrate that helped to reduce the charging phenomenon. The aluminum coated silicon was also preferred over other conductive substrates due to its flat surface profile. A $2 \mu \mathrm{l}$ drop of purified PM, suspended in ddi water, was placed on top of the substrate. A glass microscope slide was placed on top of the PM drop with a 25 $\mu \mathrm{m}$ gap between the glass slide and the aluminized silicon substrate. After the top glass was in place, the PM was allowed to dry under ambient conditions for 24 hours. The result was a dried film of non-oriented purple membrane that adhered to the aluminized silicon wafer.

After the specimen was prepared, it was placed in the FIB and four trenches were machined into the specimen. Each trench was approximately $10 \mu \mathrm{m}$ wide by $90 \mu \mathrm{m}$ long. Included below are three images captured using the FIB in image capture mode. Figure 9 shows the interface between the PM film and the aluminum. The aluminum is on the left of the image while the purple membrane is on the right of the image. The interface of the PM and the aluminized silicon substrate was selected as the machining site so that it was

possible to visually ascertain if the beam had completely machined through the PM film and reached the aluminum substrate below. 


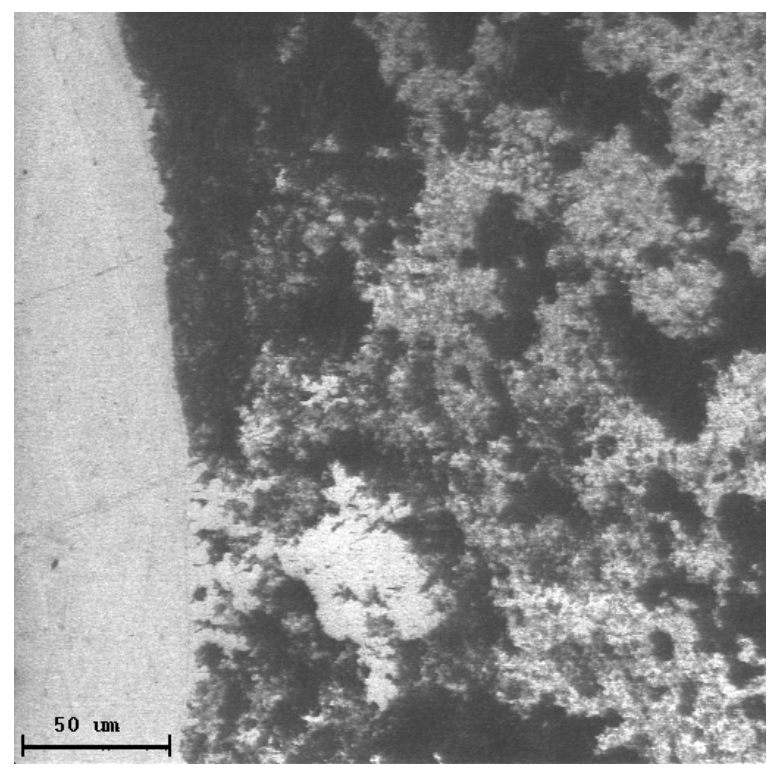

Figure 9: FIB image of PM Aluminum interface ( $\mathrm{Al}$ on left, $\mathrm{PM}$ film on right). Scale bar $=50 \mu \mathrm{m}$.

Two trenches, each $10 \mu \mathrm{m}$ x $90 \mu \mathrm{m}$ were created using the vector scan controller software on the FIB. Two separate machining procedures were performed. The first procedure used the M1-50 beam to machine the trenches. The total machining time was 27 minutes at a beam current of $0.154 \mathrm{nA}$. A dwell time of $100 \mu$ s per pixel was selected, and the beam rastered over the two trenches 2000 times (frame number). Figure 10 shows the results of this first machining procedure. As can be seen in Figure 10, the beam failed to completely machine through the PM film using this machining setup. A clear interface between the PM region and the aluminum region could still be seen in the trenches. 


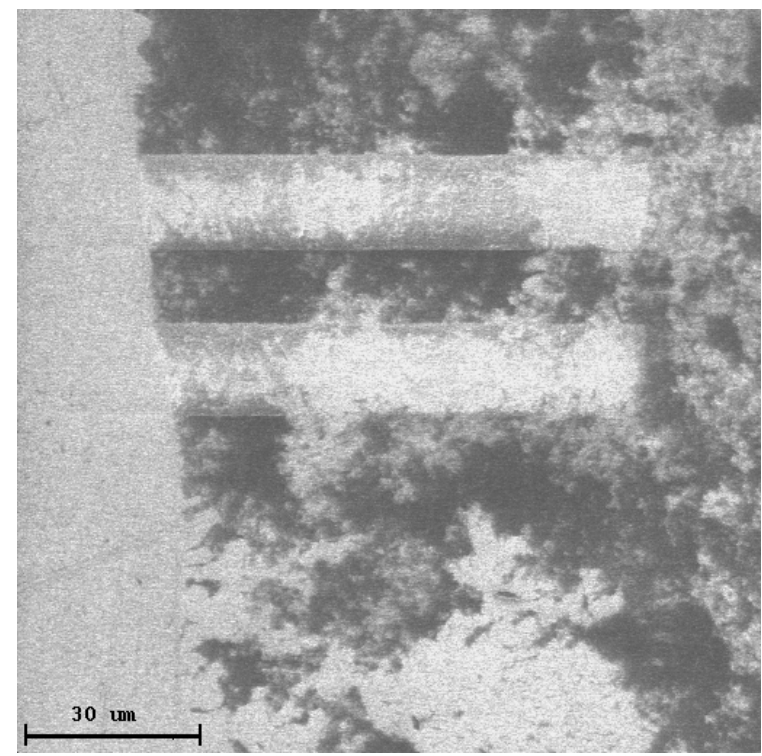

Figure 10: FIB image of two parallel trenches machined in the Al-PM interface using the M1-50 beam. Scale bar $=30 \mu \mathrm{m}$.

A second machining procedure, using the higher current density M1-100 beam, was performed in an attempt to completely machine through the PM film and into the aluminum substrate. The beam current was $0.602 \mathrm{nA}$ and the machining time was again 27 minutes. A dwell time of $100 \mu$ s per pixel was selected, and the beam rastered over the two trenches 2000 times. The results of this machining procedure can be seen in Figure 11. No interface between the aluminum and the PM film can be seen. This indicated that the FIB successfully machined through the PM layer. 


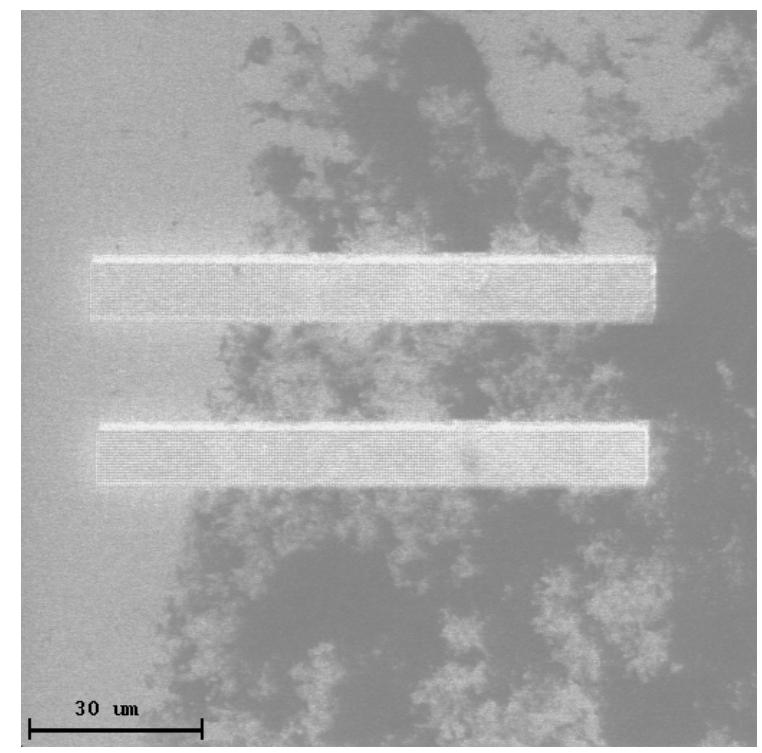

Figure 11: FIB image of two parallel trenches machined in the Al-PM interface using the M1-100 beam. Scale bar $=30 \mu \mathrm{m}$.

After the sample was removed from the FIB, it was analyzed using a white light interferometric microscope (IFM). This type of microscope uses the constructive and destructive interference of two different path lengths of light to obtain topographical information from a specimen. In order for an IFM to capture data, the sample surface must be reflective. It was found that the PM film did not adequately reflect light, and thus the entire sample was sputter coated with $\sim 5 \mathrm{~nm}$ of gold using an Anatech Hummer 6.2 sputtering system. After the sample was coated, it was placed in the IFM. It was discovered that the PM layer at the PM-Al interface was too thin to definitively show that the PM film was being removed. To solve this problem, a new sample was prepared.

A $10 \mu \mathrm{l}$ sample of PM suspended in ddi water was placed on a glass slide that had previously been coated with Indium Tin Oxide. A second ITO coated glass slide was placed on top of the drop, with a $0.967 \mathrm{~mm}$ gap between the two slides. A voltage of 3.9 $\mathrm{V}$ was applied between the two plates for 60 seconds. The drop was then allowed to dry under ambient conditions. The sample was placed in the FIB, and several patterns were machined into the PM film. Two sets of double trenches were machined, each trench 
measuring $40 \mu \mathrm{m} \times 10 \mu \mathrm{m}$. The sample was sputter coated with $15 \mathrm{~nm}$ of gold using the Hummer 6.2 sputtering system in order to facilitate IFM analysis of the specimen.

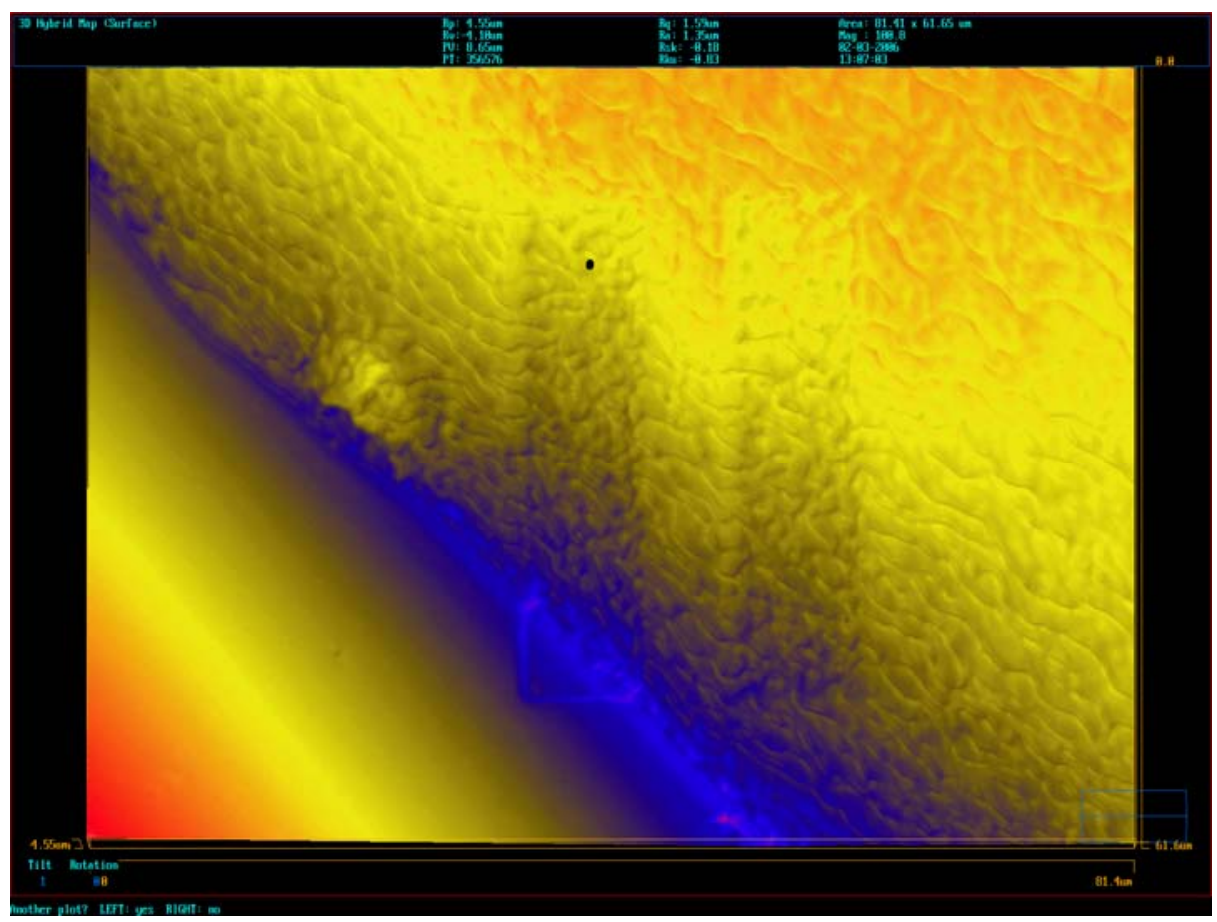

Figure 12: Interferometric Microscope image of two parallel trenches machined using a FIB machine with a M1-50 beam in a dried PM film on Indium Tin Oxide coated glass slide. Smooth surface on the bottom left quadrant is ITO while the wavy surface is the dried PM film.

Figure 12 shows the first set of double trenches machined into the bR film captured using the IFM. These trenches were positioned at the transition from the ITO coated glass substrate and the PM film. An M1-50 beam was used with a dwell time of $100 \mu \mathrm{s}$ and a frame number of 2000. The trenches took approximately 11 minutes to machine. According to IFM analysis, the depth of the trenches measured $0.434 \mu \mathrm{m}$. The vector scan controller was used to generate the patterns shown in Figure 12 and Figure 13. The PM film appears rough in the image while the ITO surface appears smooth. 

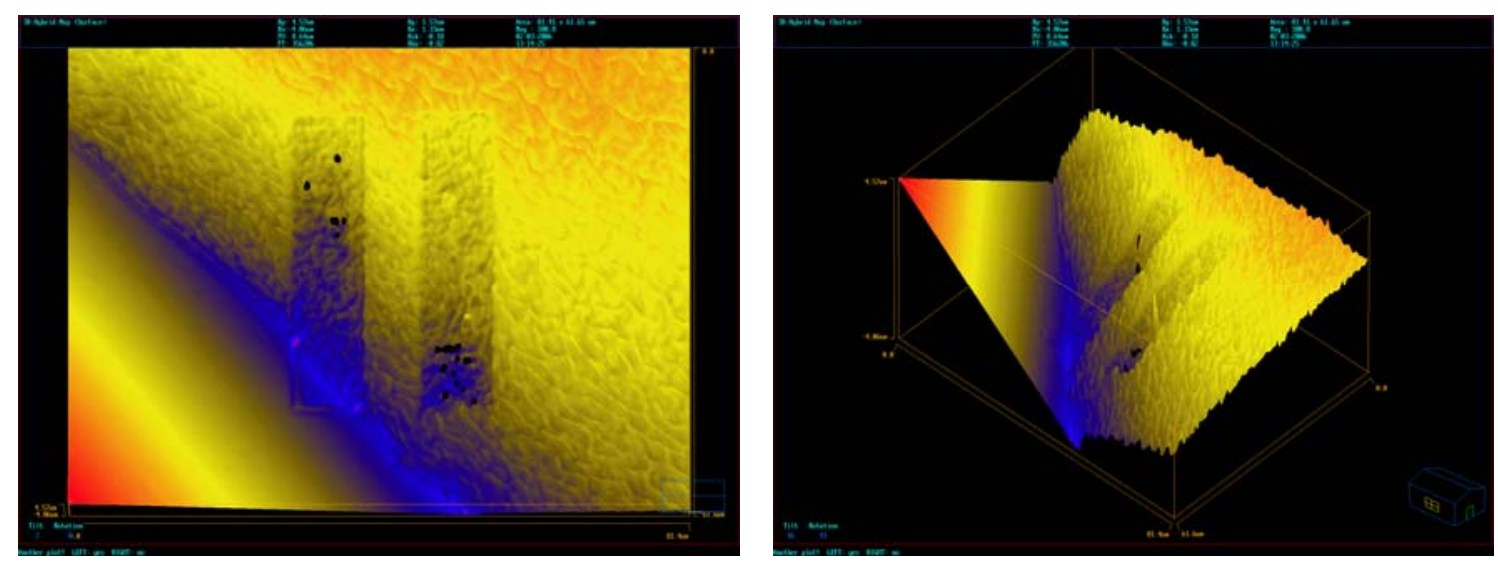

Figure 13: Interferometric Microscope images of two parallel trenches machined using a FIB machine with a M1-100 beam in a dried PM film on Indium Tin Oxide coated glass slide. Smooth surface on the bottom left quadrant is ITO while the wavy surface is the dried PM film.

Figure 13 shows the second set of double trenches in both 2D and 3D views. An M1100 beam was used with identical dwell time and frame number to the first set of trenches. This pattern took approximately 11 minutes to machine. This set of trenches was found to be deeper than those machined using the M1-50 beam, measuring approximately $0.80 \mu \mathrm{m}$. This increase in machining depth was expected due to the fact that the M1-50 beam only uses approximately one fourth of the beam current used by the M1-100 beam.

A second study was performed that explored the surface topography created in the FIB machined areas of dried PM films. An oriented PM film was first created on a brass substrate by electrodeposition. The sample was allowed to dry under ambient conditions. After the sample was dried, it was machined under various conditions in the FIB. After the machining was completed, the sample was imaged using a Hitachi S-4700 field emission scanning electron microscope (FE-SEM). It was found that a large variety of surface topographies were achievable depending upon the conditions under which the film was machined. Differences in beam size, as well as how the beam was rastered over the sample to create the desired patterning, produced different topographies within the machined area. Figure 14 shows three different patterns machined in a single 
electrodeposited PM film under different FIB conditions. Images A and B were machined using the onboard vector scan controller (8 bit spatial resolution) for pattern generation, while image $\mathrm{C}$ was patterned using the high resolution (32 bit spatial resolution) Nabity scanning system.

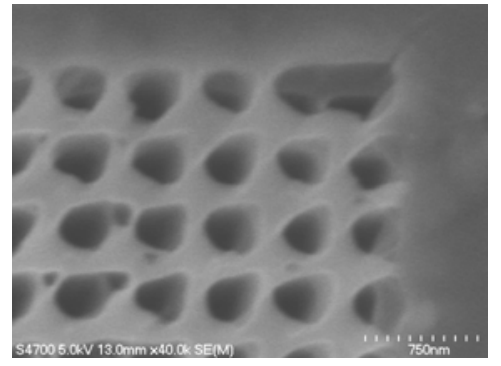

(A)

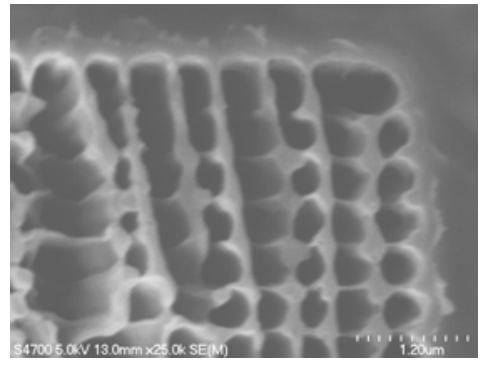

(B)

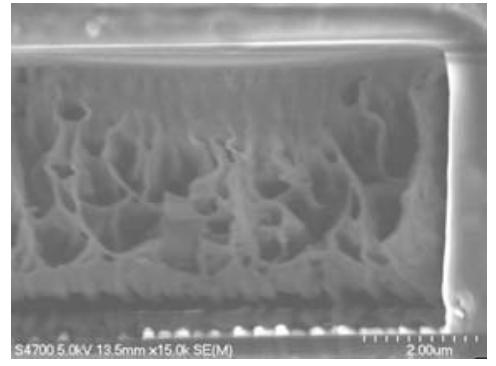

(C)

Figure 14: (A) FE-SEM image of the top right corner of a square machined into an oriented bR film using an M1-50 beam on a Hitachi FB-2000A FIB. Scale bar $=750 \mathrm{~nm}$. (B) FESEM image of the top right corner of a square machined into an oriented bR film using an M1-100 beam on a Hitachi FB-2000A FIB. Scale bar $=1.20 \mu \mathrm{m}$. (C) Right side of a trench machined into an oriented bR film using the M1-50 beam on a Hitachi FB-2000A FIB using a Nabity system to control beam rastering. Scale bar $=2.0 \mu \mathrm{m}$. All three samples used a beam dwell time of 100 microseconds.

The following images demonstrate some of the topographies achieved using the FIB machine. The operational conditions that produced each set of images are shown in the captions below the images. The PM in the images shown in Figure 15 through Figure 17 were sputter coated with $\sim 15 \mathrm{~nm}$ of gold using the Hummer 6.2 sputtering system prior to being imaged in a FE-SEM. The onboard vector scan controller was used to produce all of the patterns shown below. 

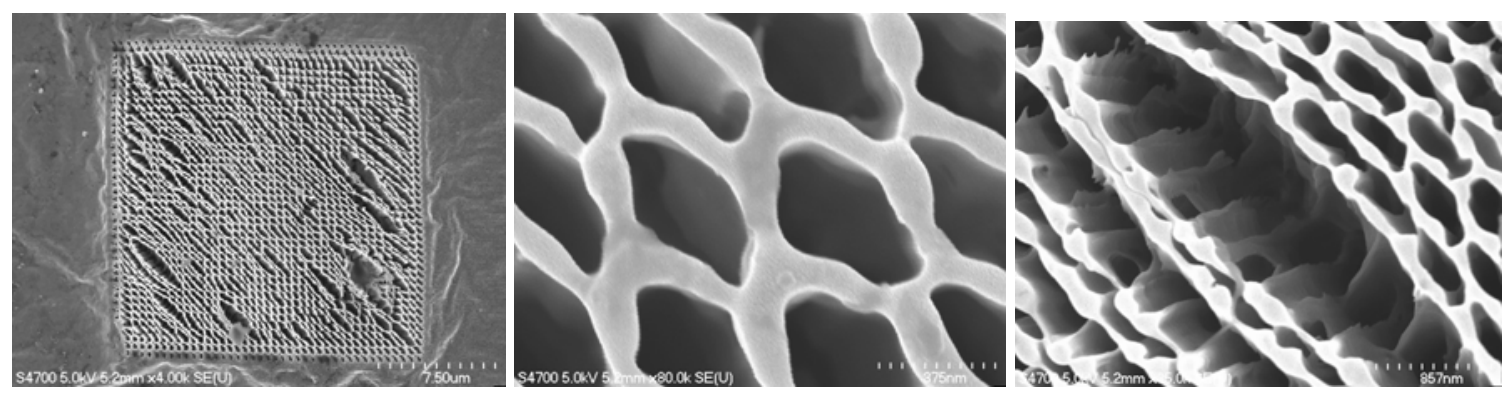

Figure 15: FE-SEM images of a $20 \mu \mathrm{m}$ x $20 \mu \mathrm{m}$ box FIB machined into a dried PM film using a M1-50 beam with a dwell time of $100 \mu$ s and a frame number of 2000 . Scale bars $=7.5 \mu \mathrm{m}, 375 \mathrm{~nm}$, and $857 \mathrm{~nm}$ respectively.
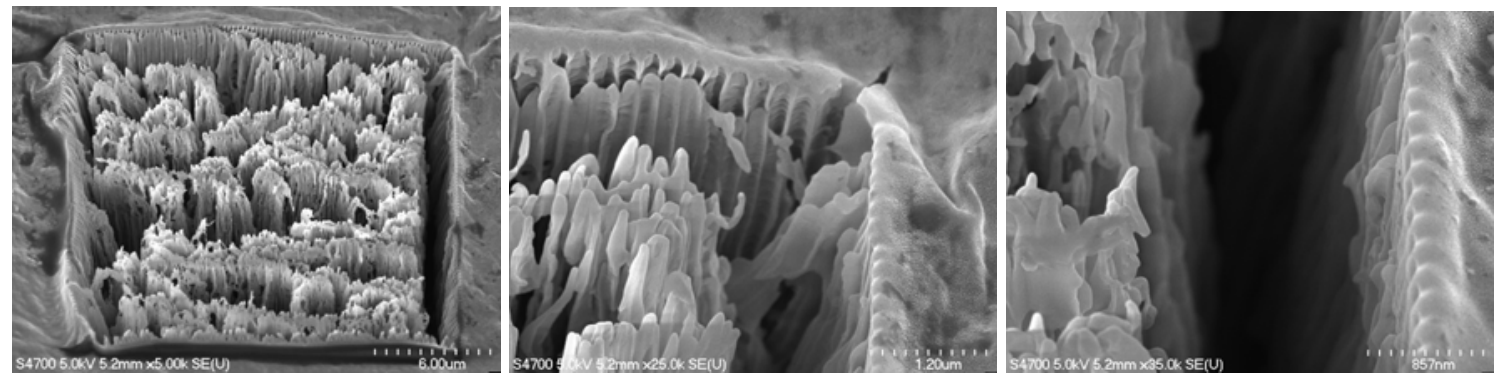

Figure 16: FE-SEM images of a $20 \mu \mathrm{m}$ x $20 \mu \mathrm{m}$ box FIB machined into a dried PM film using a M1-100 beam with a dwell time of $100 \mu$ s and a frame number of 2000 These images were taken with the FE-SEM stage tilted 30 degrees. Scale bars $=6.0 \mu \mathrm{m}, 1.2 \mu \mathrm{m}$, and $857 \mathrm{~nm}$ respectively.
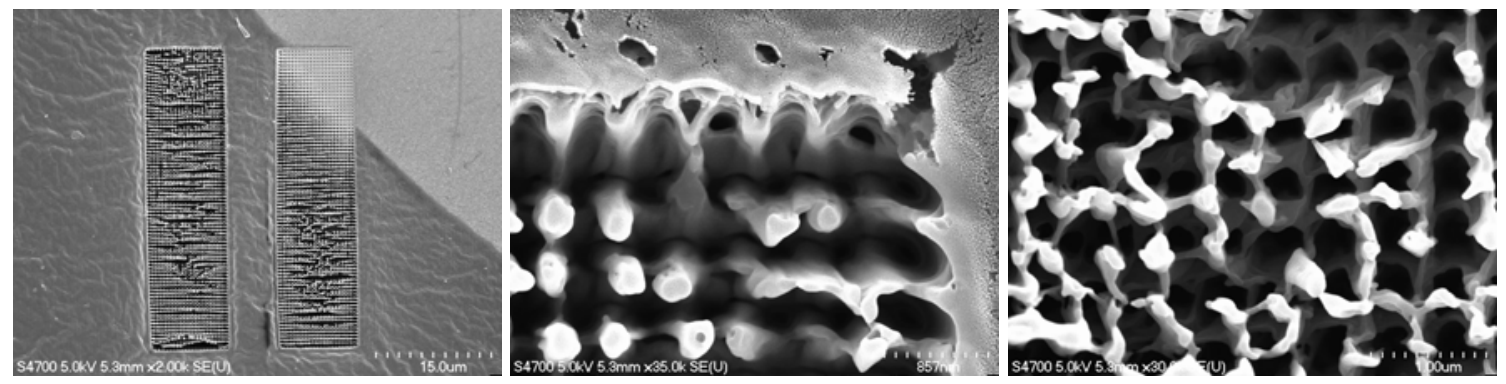

Figure 17: FE-SEM images of a $40 \mu \mathrm{m}$ x $10 \mu \mathrm{m}$ trench FIB machined into a dried PM film using a M1100 beam with a dwell time of $100 \mu$ s and a frame number of 2000 . Scale bars $=15.0 \mu \mathrm{m}, 857 \mathrm{~nm}$, and 1.0 $\mu \mathrm{m}$ respectively.

This study has shown that varying the major operational parameters of the FIB impacts the topography of the machined PM film. No operational conditions were found that resulted in a complete removal of PM from the machined area. It is likely that the 
mechanical properties of the dried PM film make the complete removal of PM films through FIB impractical. If the FIB is to be used as a PM patterning method, the complete removal of PM from the desired areas is necessary.

\subsection{Gallium Implantation Studies}

Gallium implantation into the PM sample when using FIB is of interest due to the fact that it may adversely affect the electrical properties and response of the PM film. FIB patterns by bombarding the target substrate with high-energy gallium ions. The collisions between the gallium ions and the substrate atoms results in the target atoms being displaced within the film and others sputtered away (see Figure 18). This is of particular concern because FIB machined oriented PM films are to be used in electronic systems. It is possible that the implanted gallium ions could alter the PM film's electrical properties. Theoretical studies were conducted to estimate the gallium implantation dose as well as the size of the affected area of the PM film. 


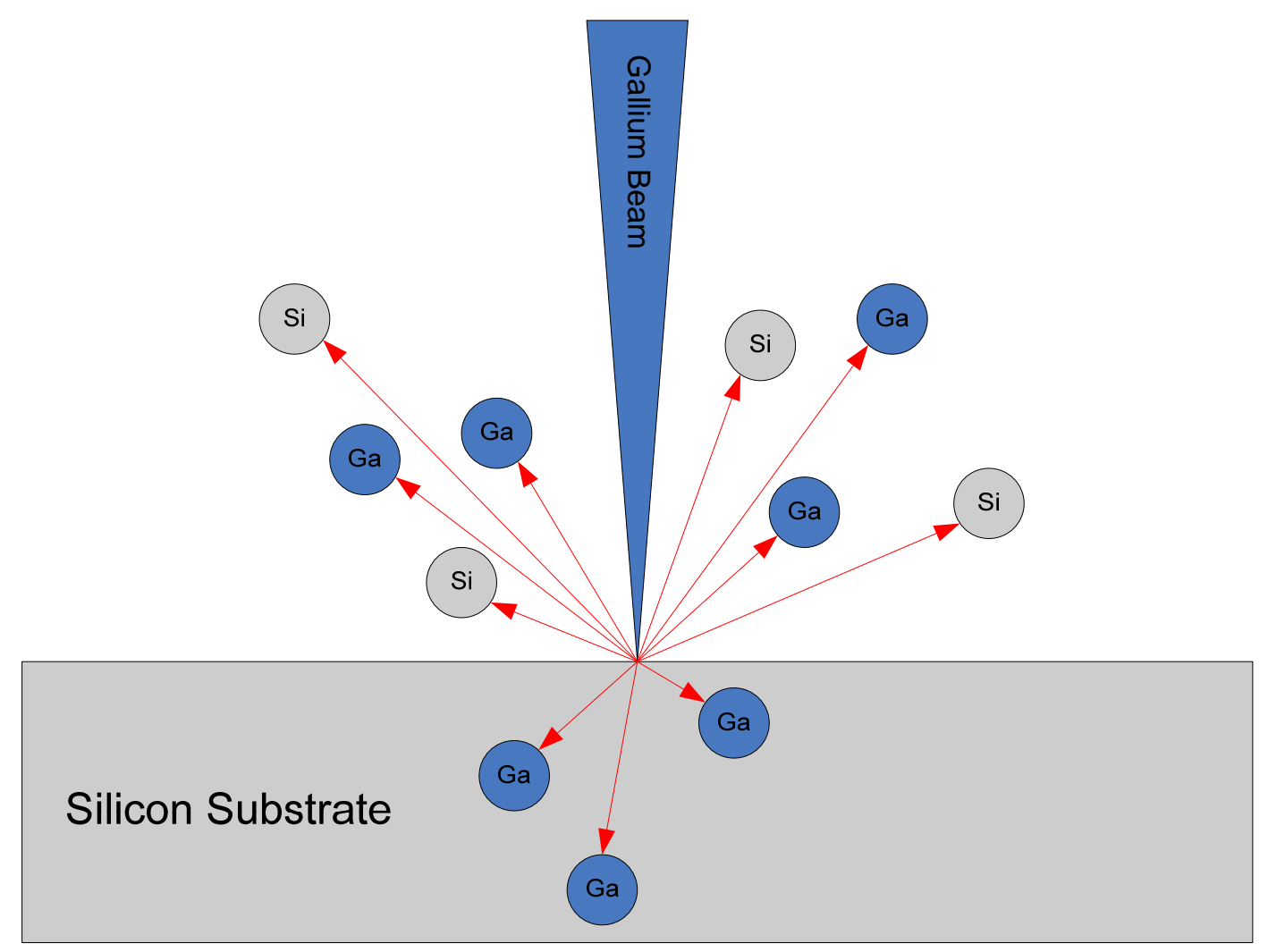

Figure 18: Illustration of gallium implantation into a silicon substrate.

Simulations were performed using SRIM simulation software to approximate the amount of gallium implantation in the PM film. The PM film was simulated with a homogenious material comprised of atomic concentrations with approximately the same ratio as found in the PM structure. The ion implantation resulting from a $30 \mathrm{keV}$ gallium beam is shown in Figure 19. These initial simulations suggest that a single beam of gallium ions will implant $80 \mathrm{~nm}$ deep with a maximum diameter of $20 \mathrm{~nm}$ into the PM film. Adding an ITO layer above the PM increases the implantation diameter from $20 \mathrm{~nm}$ to $80 \mathrm{~nm}$, due to scattering by the ITO film. 

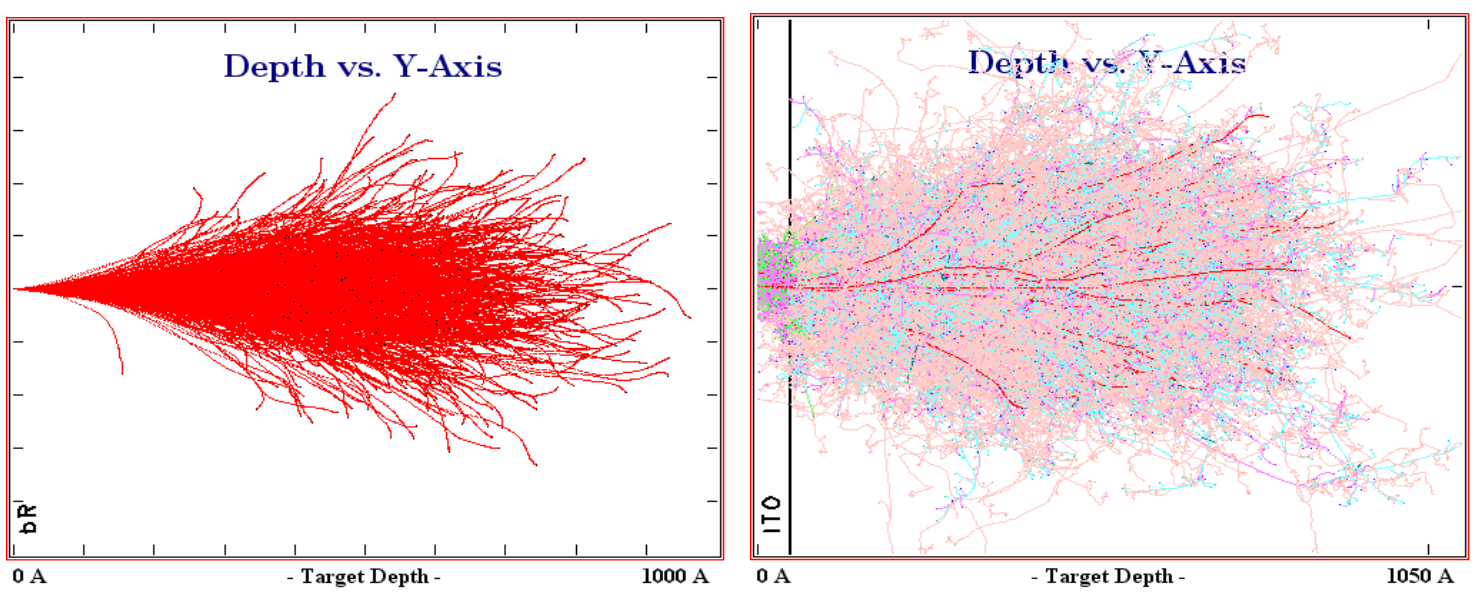

Figure 19: (Left) Ga+ implantation pattern showing 60nm deep by 20nm lateral envelope of incident ions only into bR. Scattering events (right) show an envelope $80 \mathrm{~nm}$ deep by $60 \mathrm{~nm}$ wide in a multi-layer structure of bR and 10nm of indium tin oxide (ITO) used as a top electrode. Most of the lateral scattering takes place in the ITO layer.

Experimental work has also been performed in an attempt to confirm the simulated gallium implantation findings discussed above. This was challenging due to the fact that few techniques exist that can detect trace amounts of gallium in a bulk substrate. Elemental analysis using SEM can often ascertain both the amount of an element in a bulk substrate, as well as the areas of concentration of the element. The difficulty with using this method is that it requires using a high energy electron beam to collect data on trace elements in a substrate, such as implanted gallium in a PM film. When a highenergy electron beam is focused on a PM film, the film is damaged or completely destroyed depending on the length of time the film is subjected to the beam. Because of this, an alternative method for determining the presence of gallium in a PM film was pursued.

Raman microscopy provided a way to look at the composition and structure of a material without exposing the sample to a high-energy beam. Raman microscopes use focused low energy laser light to excite the surface of a sample. A portion of the laser light is then reflected back into the detector. Most of this laser light will be the same frequency as the light emitted by the laser, however with some materials a shift in the 
wavelength occurs due to molecular vibration. This phenomenon is known as the Raman shift, and comprises a small portion of the light reflected back to the detector. It is this Raman shift that can be used to analyze various properties of a material.

The initial Raman microscopy study was performed to discover if it was possible for the Raman microscope to detect the presence of implanted gallium due to FIB machining. A polished silicon sample was used as the substrate for this experiment. One advantage of using silicon as the substrate is that the Raman spectrum for silicon has a strong peak at a wavenumber of $520.7 \mathrm{~cm}^{-1}$. Any other peaks observed in the spectrum of the sample may be identifiable as gallium. The sample was cleaned with both acetone and isopropyl alcohol (IPA) prior to machining in order to remove contaminants that could alter the Raman signature of the sample. The polished silicon sample was machined using the FIB. A $100 \mu \mathrm{m}$ by $100 \mu \mathrm{m}$ square was machined in the silicon using the high energy M1-200 beam. The dwell time was set to $100 \mu$ s and the frame number was 2000. The sample was then placed in the Raman microscope, and scans were performed in and around the machined area. A $632.81 \mathrm{~nm}$ laser source was used to irradiate the sample. The scan parameters used during this experiment were: exposure time $=2 \mathrm{sec}$, accumulation number $=5$, confocal hole $=150 \mu \mathrm{m}$, magnification $=50 \mathrm{X}$, grid $=1800$. A scan performed in the middle of the machined area can be seen in Figure 20. 


\section{Raman Spectrum}

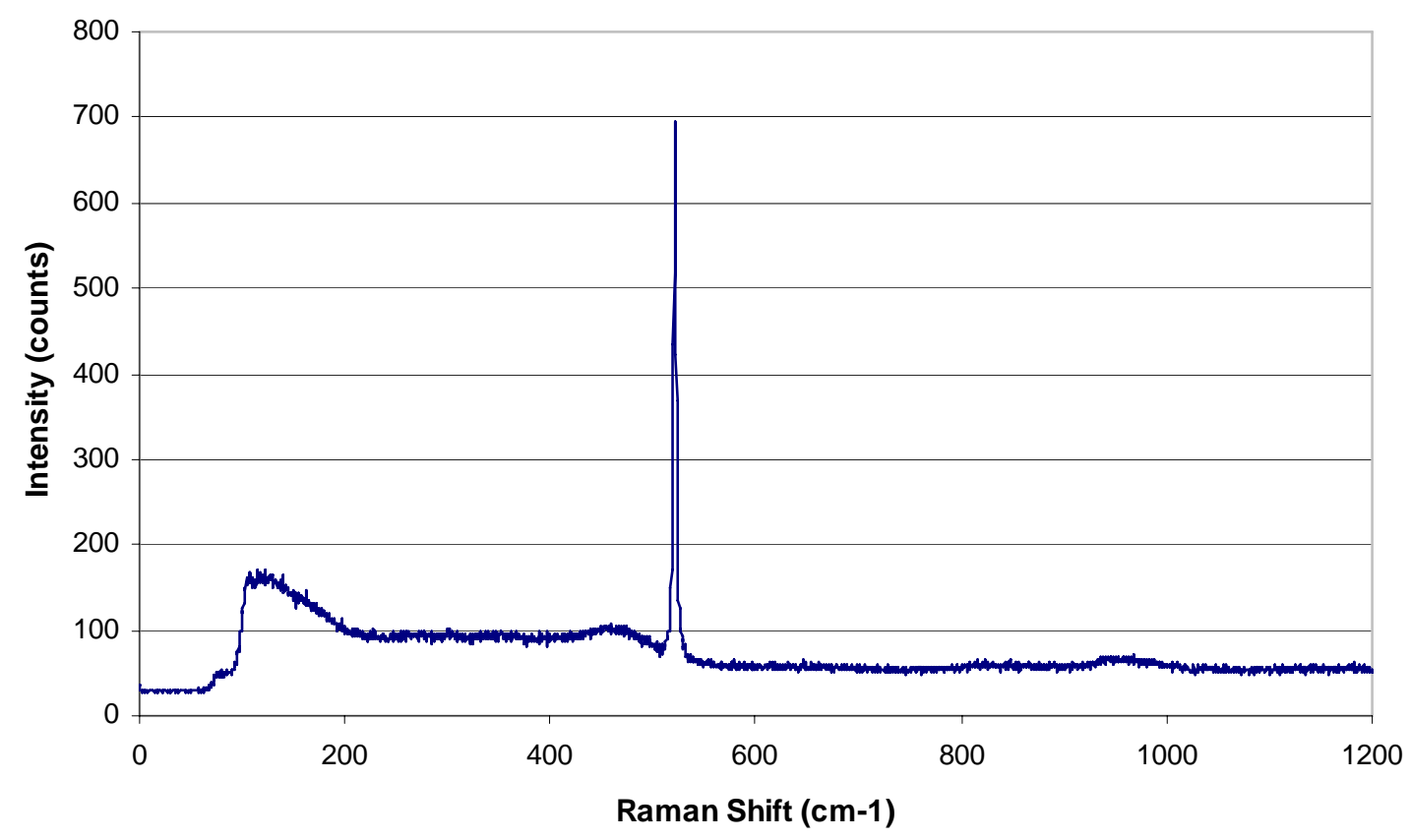

Figure 20: Raman scan performed at the approximate center of a 100 micron by 100 micron square machined in polished silicon using a FIB machine.

As seen in Figure 20, the Raman spectrum in the center of the machined area does not show any peaks other than the silicon peak at $520.7 \mathrm{~cm}^{-1}$. This was initially a discouraging result, but an interesting phenomenon was observed when scanning the areas in and around the machined area of silicon. While there was no evidence of gallium in the sample based on the Raman signature, a large variation in the intensity of the silicon peak in and around the machined region was observed. As the machined area was scanned, the number of counts associated with the silicon peak was very low when compared to scans of the same silicon sample at a location far away from the machined area. This indicated that, for some reason, the microscope was not getting as strong of a silicon signature in and around the machined area. 
After this drop in signature was observed, an experiment was performed to ascertain how large an area around the $100 \mu \mathrm{m}$ machined square showed a reduction in the silicon peak count. The microscope was set with an exposure time of 2 seconds, an accumulation number of 5, a confocal hole size of $150 \mu \mathrm{m}$, and a grid of 1800 . The first series of scans were performed at a magnification of 100X. Several scans were performed near the center of the machined square. These scans showed that the silicon peak intensity was variable within the machined area, and the typical peak intensity at $520.7 \mathrm{~cm}^{-1}$ was around 600 counts. Scans were then taken at the upper left corner of the machined square, and the silicon peak intensity was found to average around 2400 counts. Some scans were performed outside of the machined square, and showed a trend of increasing intensity as scans moved away from the machined area.

For the next portion of the experiment, the objective was changed to $50 \mathrm{X}$ magnification in order to more easily judge the distances moved between scans. Using the left corner of the machined square as the origin, measurements were taken in steps traveling away from the machined area. Because the Raman microscope's stage movements could only be controlled manually, distances moved after each scan were approximated using the scale bar visible on the camera picture of the substrate. The intensity of the measured $520.7 \mathrm{~cm}^{-1}$ peak intensity at each of these steps is shown in Figure 21. As the scans moved away from the machined area, the intensity of the 520.7 $\mathrm{cm}^{-1}$ peak gradually increased until a distance of approximately $400 \mu \mathrm{m}$ from the left corner of the machined square was reached. Beyond this distance, only small variations in the $520.7 \mathrm{~cm}^{-1}$ peak intensity were observed. 


\section{Silicon Peak Intensity vs. Distance}

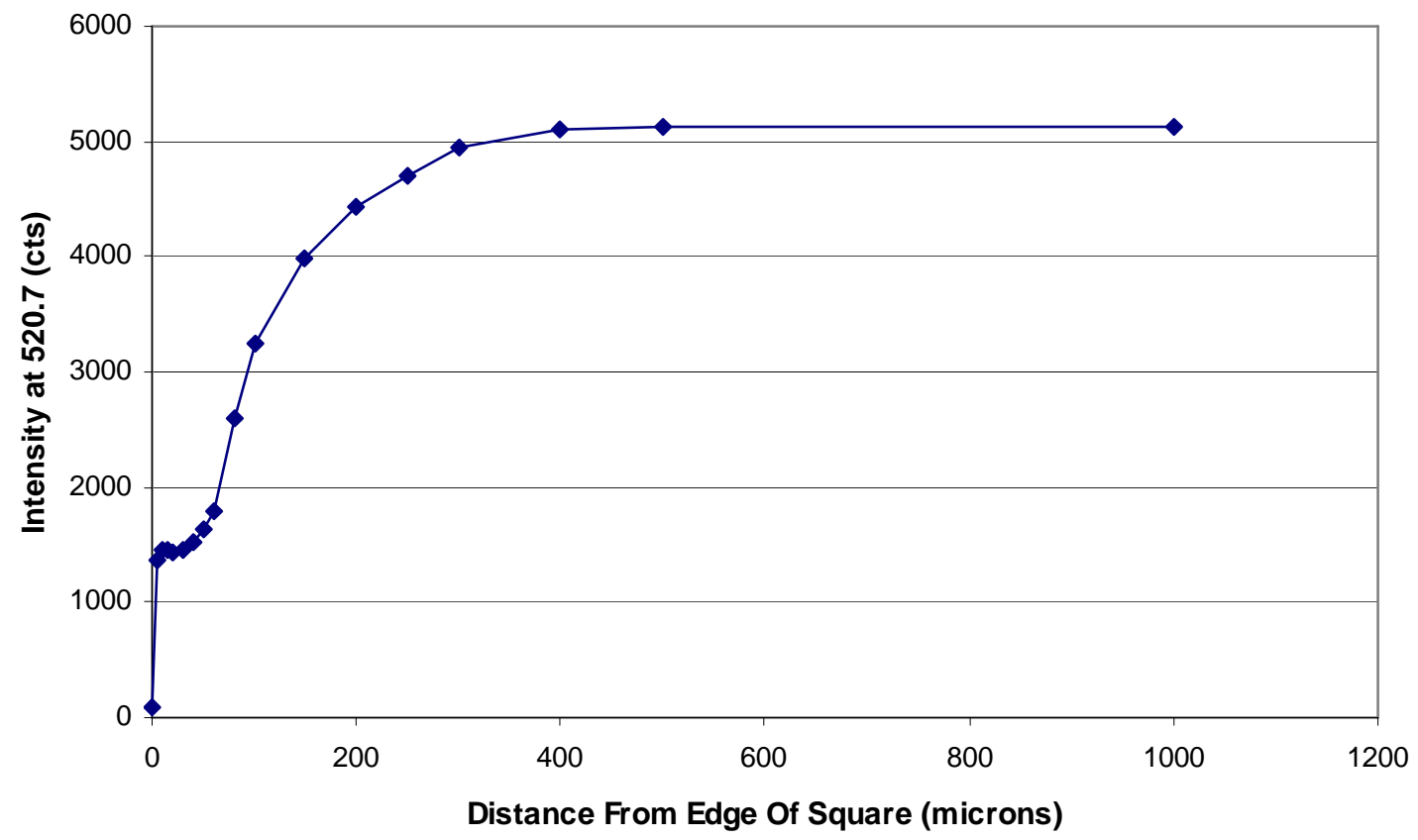

Figure 21: Intensity of Raman signal at a Raman shift of 520.7 as the scan point moves away from a corner of the 100 micron FIB machined square in polished silicon.

There are several reasons why a Raman peak will differ from one point to another. The first and most common reason has to do with the surface topography of the area of the specimen being scanned. A perfectly flat, perfectly in focus surface will yield the highest signal in a Raman microscope for a given substrate. Under these conditions, the maximum amount of laser light is reflected into the detector. As a sample surface becomes rougher, more light is scattered and less of the signal is able to reach the detector and be recorded as intensity. Further decreasing the intensity in rough areas is the fact that when the surface topography is changing rapidly, it is not possible to have the entire scanning area in focus because the depth of field of the focused area is very small. 
To confirm that the cause for the decrease in the silicon peak was not due to variations in surface roughness, an analysis of the topography of the polished silicon was performed. To obtain the surface roughness characteristics of the silicon region around the machined area, an IFM was used. Line profile roughness analyses were performed at locations both inside and outside of the machined area. It was found that the average roughness inside the machined square was approximately $13 \mathrm{~nm}$ while the average roughness outside the machined area was approximately $5.4 \mathrm{~nm}$. No detectible variation in surface roughness could be seen in areas outside the machined area. These results showed that the surface of the silicon wafer was much rougher inside the machined area, but unchanged outside the machined area. This surface analysis provided some explanation for why the silicon peak intensity was lower in the machined zone, but it does not explain the overall trend for the peak intensity to rise gradually as measurements moved away from the machined zone.

Another factor that can decrease the intensity of the peaks is simply having the laser slightly out of focus. As scans are taken across a sample that is not completely flat, the peak intensity will change as the scanning point moves away or towards the ideal focus point. One way to reduce this effect is to refocus the laser point before every measurement. Unfortunately, it is difficult to know the exact point of focus, as the process is done by manually adjusting the $\mathrm{Z}$ direction of the sample stage. However, the focus may have played a small part in some of the values obtained during the study, as the focus was not readjusted after every measurement. The focus was adjusted periodically throughout the experiment, and thus it is highly unlikely that it could have caused the long gradual trend that was observed in the peak intensity data.

A third factor that could decrease the silicon peak at $520.7 \mathrm{~cm}^{-1}$ is the presence of some other material that does not show a detectible Raman peak. It is possible that pure gallium does not emit any Raman signature in the range that is detectible by our microscope. Many of the pure metals show peaks at wave numbers of lower than 50, 
which simply shows up as noise in the Raman system used in these experiments. However, it is possible that the silicon peak intensity would be reduced because some of the laser light would hit the gallium atoms instead of the silicon atoms.

After the gallium implantation study on silicon was completed, a second study using dried PM films was performed. Two samples were prepared for this study, both of which were deposited on cleaned brass substrates. For the first sample, a $5 \mu$ drop of purified PM suspended in ddi water was allowed to dry on the brass substrate under ambient conditions for 24 hours. For the second sample, a $2 \mu \mathrm{l}$ PM suspension was deposited and oriented onto the brass substrate by electrodeposition. The two samples were then machined in the FIB, making a $100 \mu \mathrm{m}$ x $100 \mu \mathrm{m}$ square in each sample with the M1-100 beam. The dwell time was set to $100 \mu$ s and the frame number was set to 500 .

After the samples were machined, they were placed in the Raman microscope. As in the previous studies, a $632.81 \mathrm{~nm}$ laser source was used to excite the sample. The same operational parameters were used to scan the PM samples as was used to scan the silicon samples with the exception of the filter, which was set to D1 in order to reduce the intensity of the laser light on the PM samples to avoid burning. The initial results were discouraging, as the PM fluoresced under the excitation from the laser. This PM fluorescence would not allow any type of Raman signature to be captured.

For the second set of Raman scans on the machined PM films, a $325 \mathrm{~nm}$ UV laser source was used. The exposure time was set to 20 seconds with an accumulation number of 5. The confocal hole was set to $500 \mu \mathrm{m}$ and the grid was set to 2400. A 40X objective designed specifically for UV Raman microscopy was used. An example of the resulting scans on the PM film can be seen in Figure 22. Scans of both the oriented and nonoriented PM films were taken, and there was no appreciable difference in the scan results between the two samples. 
Raman Spectum of bR Film on Brass Substrate

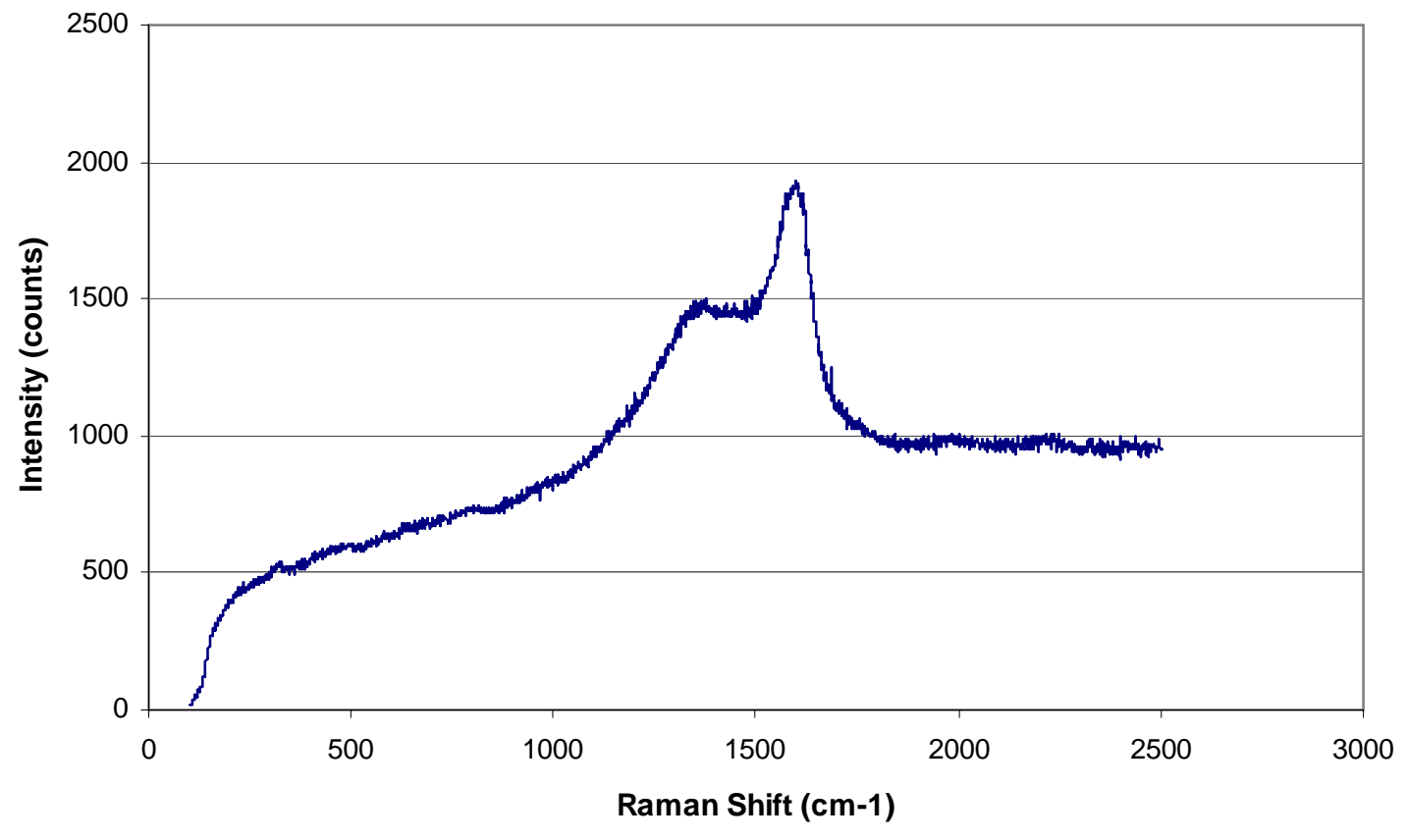

Figure 22: Raman scan of an oriented dried PM film on a brass substrate using a $325 \mathrm{~nm}$ UV laser source for excitation.

Using the $325 \mathrm{~nm}$ UV laser eliminated the fluorescence seen using the previous laser source. No Raman signature was observed when scanning areas of the brass that the PM film had not been deposited on, so the substrate had no effect on the overall signature seen above. Unfortunately, two issues arose once scanning began using the UV laser source.

The first issue was that of laser intensity. It was observed that the PM film was being damaged during the scans by the UV laser. In order to reduce the power of the laser hitting the sample, various filter settings were used. As stronger filters were applied to reduce the laser power, the effect of the laser on the PM film was decreased. It was found that scans performed using the D1 filter produced no visual damage on the PM film surface. When the intensity of the laser was reduced, it was necessary to increase 
the scanning time in order to obtain a good level of signal for each scan taken. The laser intensity cannot be filtered past the D0.6 filter, as the laser spot becomes invisible to the Raman's built in camera, which makes focusing the laser impossible.

A second and more critical issue was that of scan reproducibility. It was found through repeated scans that the main PM peak intensity at a wavenumber of approximately $1590 \mathrm{~cm}^{-1}$ varied greatly between scans. Scans were taken consecutively while moving the scan location 2 to $5 \mu \mathrm{m}$ along both the $\mathrm{x}$ and $\mathrm{y}$ axes and not altering any scan parameters, and the resulting peak intensities varied from 708 counts to 17,156 counts. This was observed in an area of the PM film far from the machined area. This variation may be due to the poor overall quality of the PM films produced. It was observed both by optical microscopy and in the FIB that both of the PM films used in this study showed cracks. These cracks exposed the brass substrate beneath the bR film, and were most likely due to poor humidity control during film drying. Because the PM films analyzed in the Raman are not continuous, as scans were taken at different locations, some of the laser light may have been reflected off the exposed brass substrate causing variations in the PM peak intensity.

Due to the large local variations in the PM peak intensity, no trends could be observed in the film as the scans approach the machined region. A method must be devised to produce dried PM films that do not show cracking when deposited onto a substrate if this analysis method is to be effective. Without a continuous PM film to scan, the variation in the film will cause too much variation in the Raman peak intensity of PM and no trends will be observable. Furthermore, changes in the Raman signature of PM due to gallium implantation do not indicate a change in the electrical properties or functionality of the bR molecules. Until it is possible to test PM films with surface areas of hundreds of microns or less, it will be difficult to draw any conclusions on the overall effect of FIB machining on PM films. 
These initial studies showed us that it is possible to pattern PM films using FIB. However, the results of these FIB experiments were discouraging. Several key issues became apparent while machining PM films. The first issue was machining time. While it proved difficult to obtain accurate machining times, it was obvious that machining PM films was a slow process. Patterning squares with dimensions of $100 \mu \mathrm{m}$ x $100 \mu \mathrm{m}$ took hours to accomplish, depending on the FIB parameters used.

The surface topography of the machined area was also disappointing for the purposes of this research. A variety of topographies were observed inside the machined areas of PM films. These topographies are discussed in further detail in subsection 3.1. While interesting, these highly irregular structures created by the FIB would make the total removal of a PM film from an electronic substrate extremely difficult.

Another issue when considering using FIB to remove areas of dried PM films is the localized heating of the film in the region surrounding the machined area. Studies have shown that dried PM films can only withstand a temperature of approximately $140^{\circ} \mathrm{C}$ before the bR undergoes a nonreversible denaturing that destroys the molecule, as well as the functionality of the PM film. When materials are exposed to the high energy gallium ion beam, the substrate undergoes localized heating at the point of beam contact as well as in an affected area surrounding the beam. The effects of these localized heating events on the functionality of PM films machined using the FIB is unknown at this time.

While the FIB machining studies have shown that it is possible to selectively remove portions of dried PM films, many more questions remain before the overall effectiveness of the process can be confirmed. It was not possible to perform any studies that address the possible loss of functionality of dried PM films machined using FIB. This is due to the fact that the areas affected by the gallium beam are very small, with the largest machined area being $100 \mu \mathrm{m}$ square. These machined areas represent an extremely small percentage of the overall surface area of a typical PM drop machined using the FIB. 
Thus, only a small area of a PM film is affected by the gallium beam, making the detection of any change difficult at the present time. This work represents the first known attempt to pattern a dried PM film using a FIB. 


\subsection{Photolithography Based Bacteriorhodopsin Patterning Technique}

The main thrust of this research was to develop a method for micropatterning oriented PM films on a CMOS electronic substrate in such a way that the functionality of the PM is preserved. The deposition and patterning of photoactive PM films onto microelectronic devices will serve as the bottom layer the proposed miniaturized detection system. This will create a light sensitive base platform that will be built upon by other researchers in this group to create the miniaturized toxin detection system discussed in Chapter 1.

Many researchers have devised methods for patterning PM films, the most common of which were discussed in the first chapter. Because the patterning process used in this research will couple PM films with microelectronic devices, the patterning process must allow for patterning the oriented PM films without damaging the underlying electronic substrate. After studying the research presented in the literature on the patterning of PM films, it was decided that the development of a new patterning method would be required.

Currently, photolithography is the fabrication method used for producing productionscale microelectronic devices. Photolithography is a well established technology that can easily be scaled up to produce large volumes of components cost effectively. These characteristics make photolithography an attractive technology to employ in a novel PM film patterning technique.

\subsection{Initial Development of the PBBPT Process}

The photolithography-based bacteriorhodopsin patterning technique (PBBPT) involves modifying standard photolithographic process steps to allow the deposition of 
PM films while preserving the functionality of the bR contained in the PM films. Most of the standard photolithography process remained unchanged. A flow chart representation of the basics of the PBBPT process is shown in Figure 23.

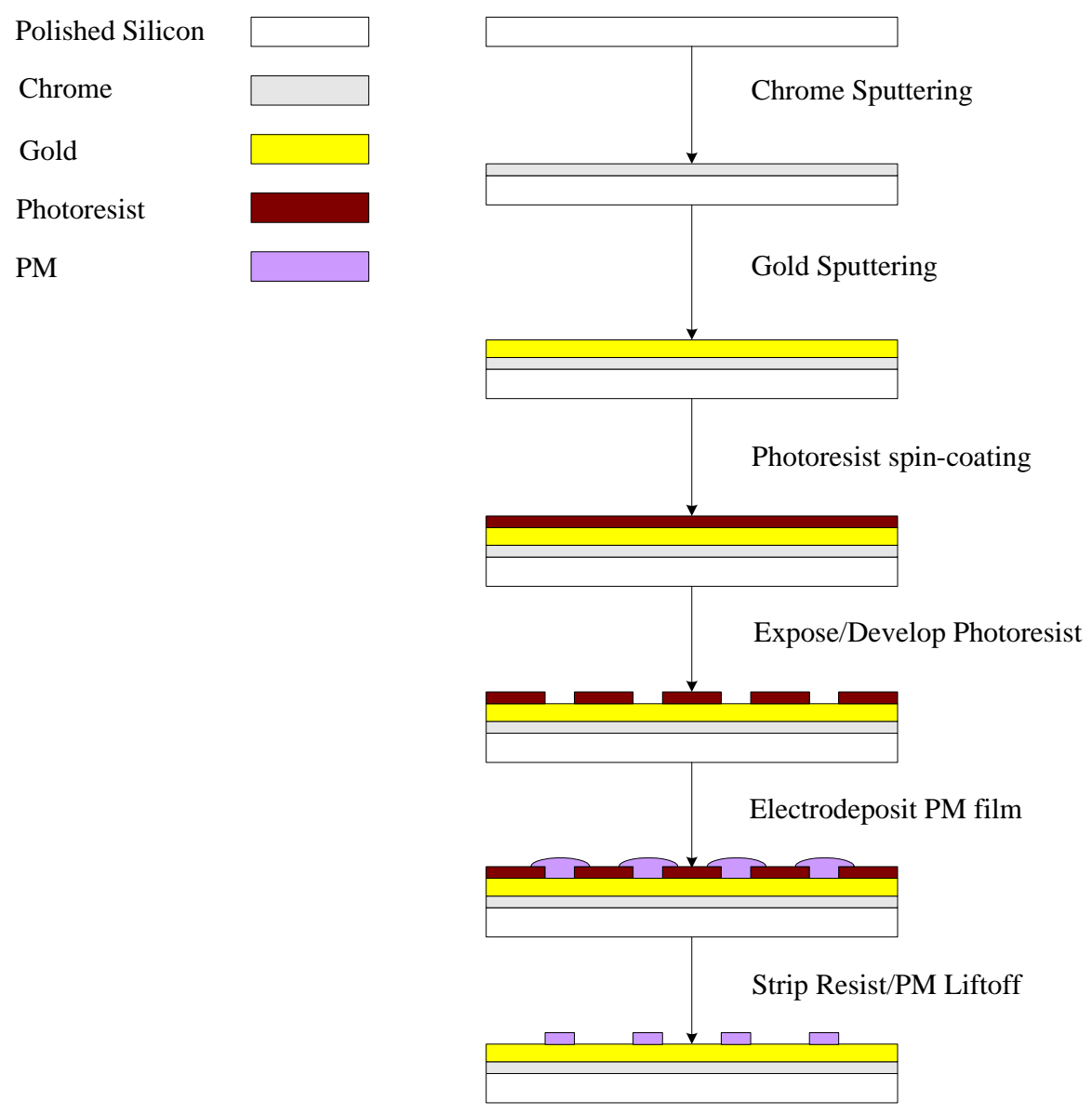

Figure 23: Basic PBBPT process flow resulting in a patterned PM film over a uniform gold coated silicon wafer.

This process flow allows for PM to be patterned onto a uniform conductive substrate. The goal of this process was to ascertain if patterning electrodeposited PM films would be possible using photolithographic techniques.

The PBBPT utilizes the liftoff process commonly used in photolithography. This process flow exposes the PM film to the final liftoff step of the process. It was decided 
not to pursue an etching process flow in which the PM film would first be deposited onto a substrate, and then preferentially removed through a lithographic etching process. An etching process flow would expose the PM film to all of the lithographic process steps, including soft and hard bakes, PM exposure and development, etching, and liftoff. The PM film must retain its photoelectric functionality if the patterning process is to be successful. Because an etching process flow would expose the PM film to harsh conditions (elevated temperatures during bakes, exposure to high intensity UV light, exposure to photoresist development chemicals), this process flow was not pursued.

\subsubsection{PBBPT Using Resist Developer to Perform Liftoff}

A four inch polished silicon wafer was used as the starting substrate. The wafer was cleaned using the piranha cleaning process. Hydrogen peroxide was added to sulphuric acid at a ratio of 1:10 to produce the piranha solution. The wafer was submerged for 5 minutes in the solution to remove any organic contaminants present on the wafer. The wafer was then thoroughly washed (5 times) in ddi water. This chemical cleaning was performed in the cleanroom. After the wafer had been cleaned, it was removed from the cleanroom and placed in a Perkin-Elmer model 6J RF sputter deposition system. An Argon flow of 10 sccm and an RF power of 100 Watts were used for all $\mathrm{Cr}$ and $\mathrm{Au}$ depositions discussed in this document. The wafer was coated with $10 \mathrm{~nm}$ of chrome followed by $50 \mathrm{~nm}$ of gold.

After the wafer had been sputter coated, it was brought back into the cleanroom and cleaned with acetone followed by isopropyl alcohol (IPA). The wafer was then coated with Futurrex 2000A photoresist using a spin coater. Futurrex 2000A is a positive photoresist, and thus areas of the resist that have been exposed to UV light become less resistant to chemical removal by the resist developer. The photoresist was spun on at 4000 RPM for 60 seconds. Immediately after the photoresist was applied, the wafer was 
soft baked by being placed on a hot plate set to $140^{\circ} \mathrm{C}$ for 60 seconds. The photomask containing the desired pattern along with the wafer was then placed into an EVG EV620 mask aligner for photoresist exposure. The mask was brought into contact with the substrate using soft contact mode, and the photoresist was be exposed to the high intensity ultra-violet light source $(350 \mathrm{~nm}-450 \mathrm{~nm})$ for 12 seconds. The wafer was then removed from the aligner system.

Futurrex RD-6 photoresist developer was used to develop the wafer. The resist developer was poured into a Teflon trough, and the wafer was submerged in the developer. The trough was gently agitated in order to facilitate the resist development. The wafer was removed from the resist developer and immediately placed in a beaker of ddi water after 15 seconds of exposure to the photoresist developer. After thoroughly rinsing the wafer, the patterned photoresist was evaluated using an optical microscope in the cleanroom. After it was confirmed that a quality pattern had been achieved in the photoresist, the wafer was removed from the cleanroom to perform the PM deposition step.

The PBBPT allows the PM film to be deposited using any of the deposition methods discussed in Section 1.3. For all PBBPT studies, electrodeposition was used to orient and deposit PM films. The ability to quickly deposit oriented films with thicknesses ranging from 100's of nanometers to 10's of microns made electrodeposition the most advantageous deposition technique for this work. In the process shown in Figure 23, the photoresist acts as an electrical insulator. Because of this, a photolithographicallypatterned conductive substrate will only be electrically accessible in the areas where the photoresist has been removed. Electrodeposition can be used to deposit PM films onto the areas of a substrate that are electrically accessible. Any excess PM will be lifted off when the photoresist is stripped away. 
After the wafer was patterned, an oriented PM film was deposited by electrodeposition. Several drops of ddi water containing different concentrations of suspended PM fragments, ranging from an optical density of $\sim 6.5$ down to an optical density $\sim 0.203$, were dispensed onto areas of the wafer where the gold was exposed. Each drop contained a total volume of $25 \mu \mathrm{l}$. The gold bottom layer acted as the anode while a brass plate acted as the cathode. The brass cathode was placed on top of each drop individually with a $\sim 1.2 \mathrm{~mm}$ spacer placed between the brass electrode and the substrate. This separation distance allowed the PM liquid suspension to contact the cathode. After the cathode was in place, a voltage of $3.3 \mathrm{~V}$ was applied for 60 seconds, removed for 20 seconds, and then applied again for another 20 seconds. This application of voltage caused PM fragments in the liquid suspension to be drawn down to the positive electrode and deposited as a film having some level of orientation. Each PM concentration was electrodeposited individually.

Immediately following each electrodeposition, the wafer was gently washed in a bath of ddi water to remove any PM fragments that had not been deposited onto the wafer. The wafer was then dried using nitrogen. This process was repeated for 6 different concentrations of PM suspension. Because the photoresist present over much of the wafer was electrically insulating, PM was only deposited on the exposed gold areas and a small area of photoresist surrounding the exposed gold areas. Each drop of PM suspension was deposited on areas of the wafer containing the same repeating pattern of circles and squares in the photoresist.

After all of the depositions were completed, the wafer was studied under a Nikon Optiphot 200 optical microscope equipped with a digital camera. Figure 24 shows two of the images captured using the microscope. The left image in Figure 24 shows the first deposition, representing the film created by electrodeposition of a PM drop with an optical density of 6.5. The resulting patterned film can be seen, not only in the exposed 
gold regions, but also in a radius surrounding each gold area. The right image in Figure 24 shows the final deposition of a PM drop with an optical density of 0.203 .
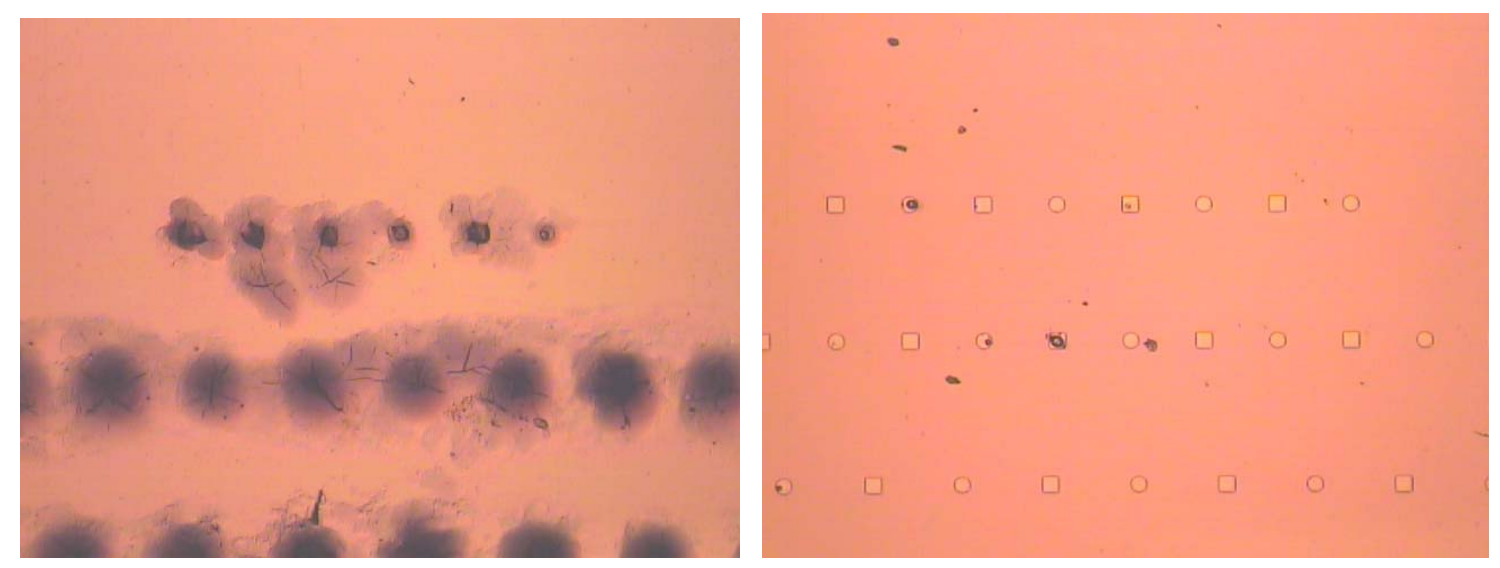

Figure 24: Optical microscope images (5X magnification) of oriented PM films (right $=$ OD 6.5, left $=$ OD .203) electrodeposited onto a gold coated Si wafer patterned using photolithography.

After the PM film deposition was completed, the remaining photoresist was stripped from the wafer. This was accomplished by placing the wafer in Futurrex RD-6 photoresist developer for 2 minutes. Futurrex RD-6 removes the light exposed areas of the photoresist at a much faster rate than the rest of the non-exposed areas, creating a pattern. However, if a wafer is left in the photoresist developer for a longer period of time than is required to develop the wafer, all of the photoresist will be removed. Once the wafer was stripped of photoresist, it was analyzed under an optical microscope to see if the alternating square and circle patterns made in the resist by the original development process would still be visible. Figure 25 through Figure 28 show the results of depositions 1, 2, 5, and 6 respectively beginning with the most concentrated PM suspension and ending with the least concentrated PM suspension. 

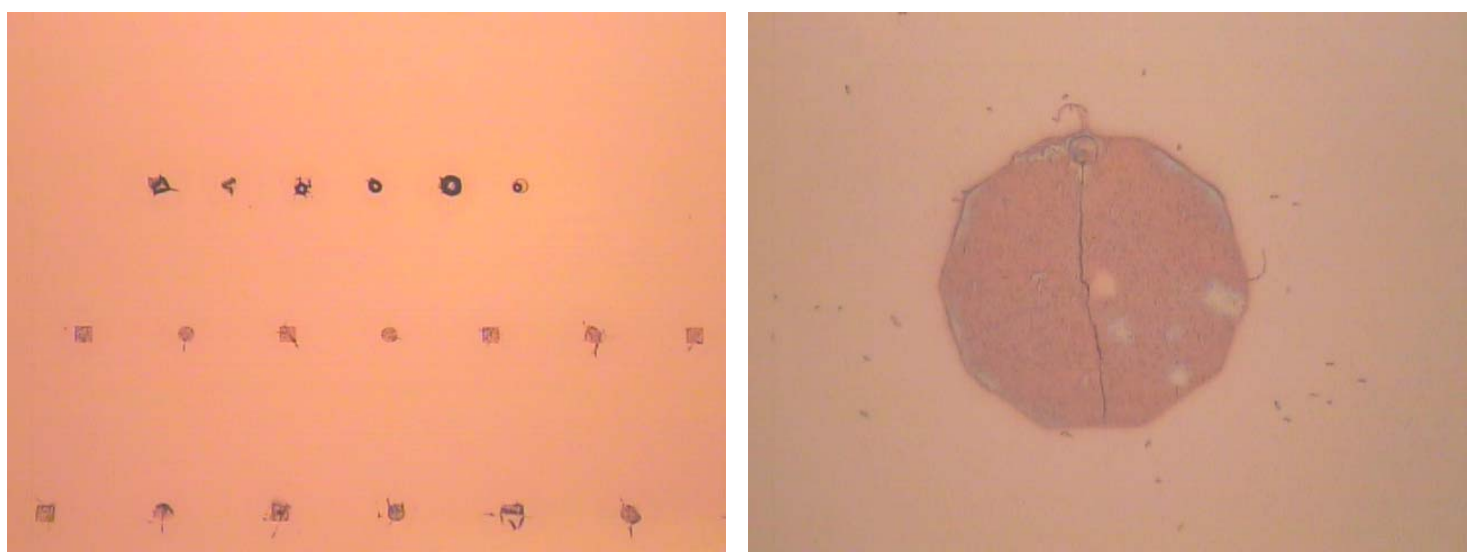

Figure 25: Optical microscope images of oriented PM film patterns $(\mathrm{OD}=6.5)$ electrodeposited onto a gold coated silicon wafer patterned by photolithography. $($ left $=5 X$, right $=100 X)$
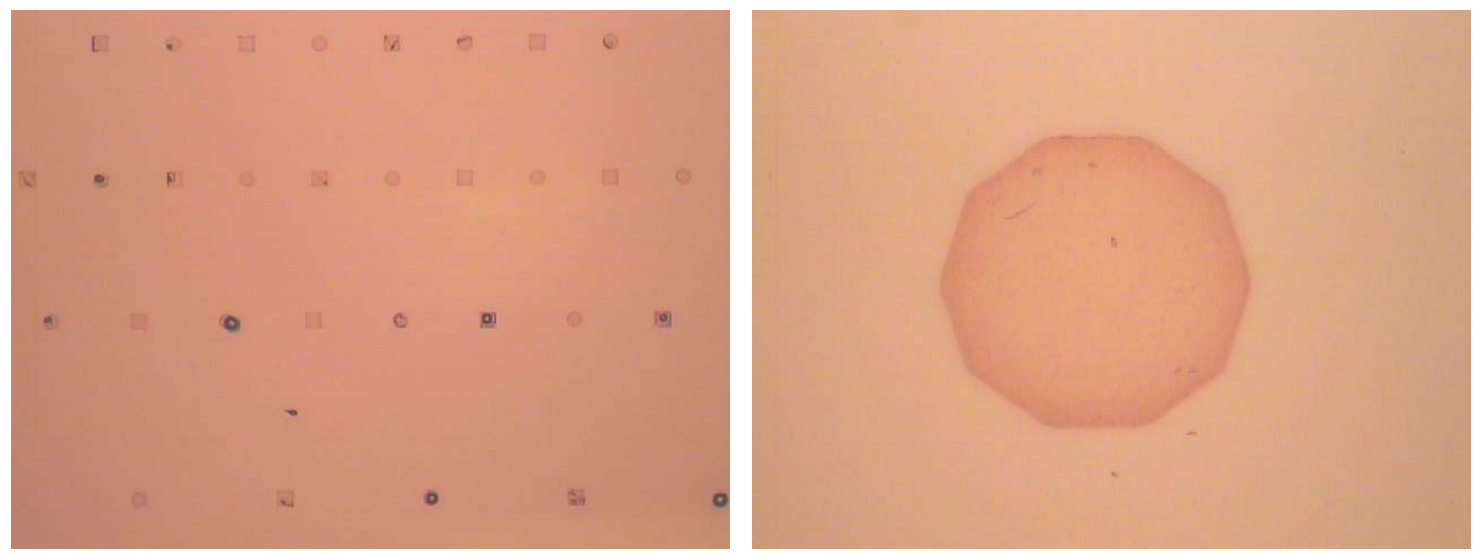

Figure 26: Optical microscope images of oriented PM film patterns $(\mathrm{OD}=3.25)$ electrodeposited onto a gold coated silicon wafer patterned by photolithography. $($ left $=5 \mathrm{X}$, right $=100 \mathrm{X})$ 

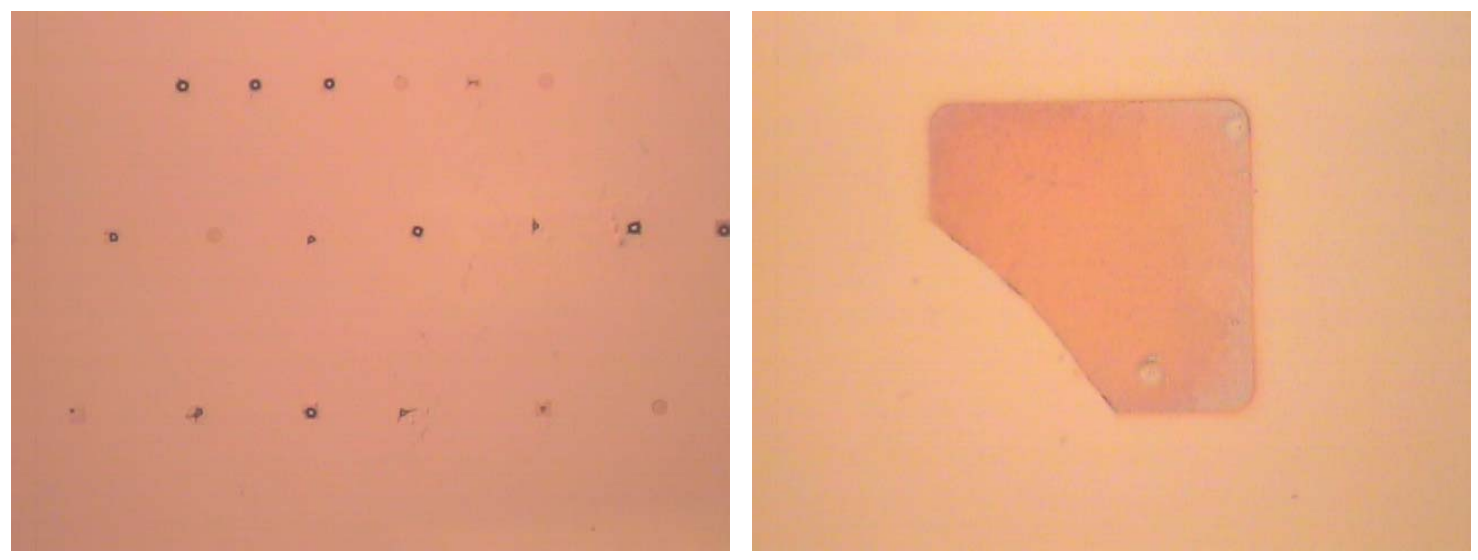

Figure 27: Optical microscope images of oriented PM film patterns $(\mathrm{OD}=0.41)$ electrodeposited onto a gold coated silicon wafer patterned by photolithography. $($ left $=5 \mathrm{X}$, right $=100 \mathrm{X})$
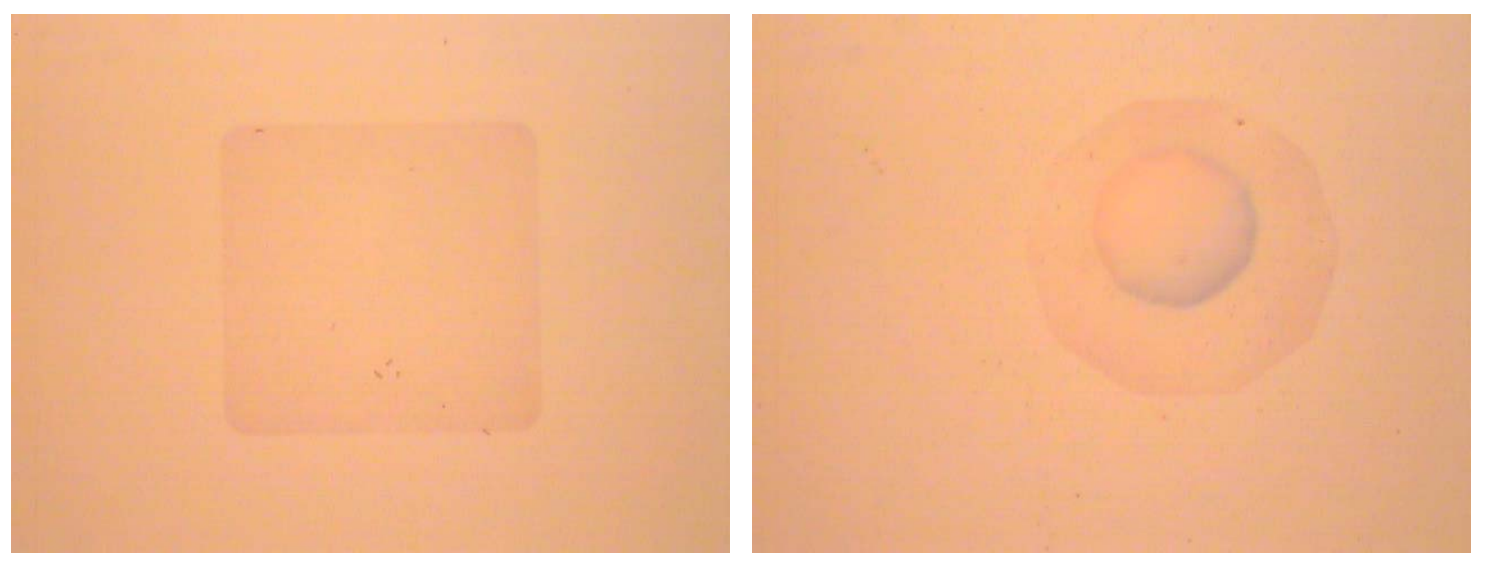

Figure 28: Optical microscope images of oriented PM film patterns $(\mathrm{OD}=0.203)$ electrodeposited onto a gold coated silicon wafer patterned by photolithography. (left $=100 \mathrm{X}$, right $=100 \mathrm{X})$

As can be seen from the images, the initial patterning results appeared very encouraging. Because all of the resist had been removed, the only patterns remaining on the gold wafer were deposited PM films. The PM films surrounding the patterned areas seen on some of the more dense depositions had been removed along with the photoresist. Some variation in height between the gold surface and the patterns seen above was observed when modulating the focus on the optical microscope.

To confirm that the features seen in Figure 25 through Figure 28 had some height above the gold surface, the features were analyzed using an IFM. To image this sample 
in the IFM, the sample was coated with a second layer of gold $20 \mathrm{~nm}$ thick. IFM analysis of the sample produced mixed results. The analysis showed some height component to the patterns shown in Figure 25 through Figure 28. A single circle in sample 1 (Figure 25) was shown to be approximately $60 \mu \mathrm{m}$ in diameter and approximately $500 \mathrm{~nm}$ thick. A single circle from sample 6 (Figure 28) was shown to have a thickness of only approximately $8 \mathrm{~nm}$. While it was encouraging that these patterns had some height, much thicker films were expected based on the optical densities of PM deposited. Nevertheless, these results show that some PM film had been deposited in the exposed gold regions of the patterned wafer, and after the photoresist was stripped, high resolution patterns were achieved. This represents the first time photolithography has been used as a PM film patterning tool.

One question left unanswered by the IFM study was why the PM films were thinner than expected. One possibility was that the Futurrex RD-6 developer not only attacked the resist, but also the dried PM films. To test this hypothesis, a drop of PM fragments suspended in ddi water was dried on an ITO coated glass slide. The slide was dipped in a jar containing the resist developer and gently agitated. The result was a complete removal of the PM film from the ITO coated glass slide within 20 seconds. This result provided an excellent explanation for why such thin PM films were observed once the resist was stripped, and indicated that alternative photoresist stripping chemicals must be explored.

Several additional chemicals capable of removing photoresist were available in the cleanroom. The first additional chemical solution used was Shipley MF-319 resist developer. This chemical solution was intended for development of Shipley photoresists, and was capable of removing Futurrex 2000A photoresist. To test the effects of MF-319 on PM films, a dried PM film that was previously electrodeposited onto an ITO coated glass substrate was submerged in the solution for 30 seconds. During this time, the PM film was removed from the substrate. An identical test was performed using acetone 
instead of MF-319. After 30 minutes, the dried PM film showed no signs of being removed from the substrate by the acetone. This was a promising result, as acetone is very effective at removing photoresist from a substrate. It should be noted that rinsing a sample in water after the acetone liftoff bath resulted in the removal of the PM film. Because of this, pure acetone was used for the liftoff, and the sample was allowed to air dry.

\subsubsection{PBBPT Using Acetone to Perform Liftoff}

As discussed in the previous section, submerging a dried PM film in acetone did not result in the visible removal of the film from the substrate. To confirm this observed result, a study investigating the effects of an acetone bath on a dried PM film was performed. A $15 \mu \mathrm{l}$ drop of PM suspended in ddi water $(15 \mathrm{mg} / \mathrm{ml})$ was electrodeposited onto a piece of gold coated silicon, forming a thick circular film. An electric field was created between the gold coated silicon top plate and the substrate by applying 4.0 DC volts for 60 seconds. A $1.2 \mathrm{~mm}$ spacer set the distance between the two plates. The sample was rinsed in ddi water and allowed to dry under ambient conditions (68\% humidity).

The sample was placed in a sputter coater and one half of the PM film was covered with a glass slide. $20 \mathrm{~nm}$ of gold was sputtered onto the exposed half of the PM film. The sample was then submerged in acetone and gently agitated for 30 seconds, exposing the uncoated side of the PM film to the acetone. After removal from the acetone the sample was dried with nitrogen. The sample was again placed in a sputter coater and coated with an additional $20 \mathrm{~nm}$ of gold across the entire sample. The sample was imaged using the IFM to obtain topographical data of the PM film. If the acetone caused the removal of some portion of the PM film, a step should be present between the side that was exposed to the acetone and the side that was not. 


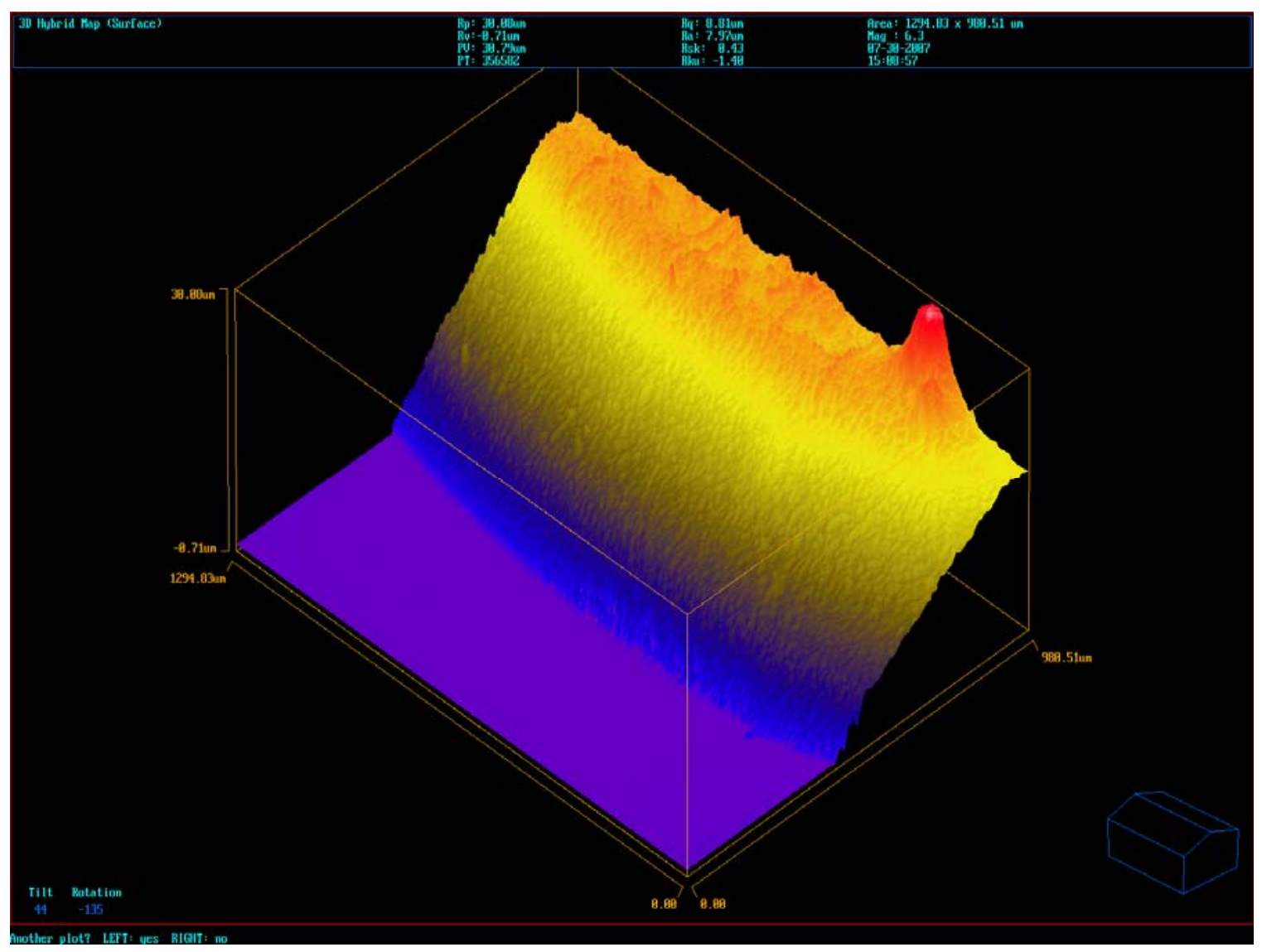

Figure 29: IFM analysis of the interface between the substrate surface and the electrodeposited PM film. This scan contains the transition between the half of the PM film exposed to acetone and the half that was not exposed to acetone.

Figure 29 shows the IFM analysis of an area of PM film containing the transition between the half of the PM film exposed to the acetone and the half that was not exposed to acetone. The flat portion in the lower left is the substrate. No step in the PM film was seen, which indicated that no detectable amount of PM film was removed upon brief exposure to acetone.

After it was determined that acetone did not physically damage or remove the PM film, the PBBPT process was carried out using acetone in the photoresist stripping step. A polished silicon wafer was sputter coated with $25 \mathrm{~nm}$ of chromium followed by 100 
nm of gold. Following the thin film deposition, the wafer was patterned in the cleanroom using Futurrex 2000A photoresist. The photolithography protocol described in Section 4.1.1 was followed to pattern the wafer. The mask used to perform lithography was comprised of alternating squares and circles approximately $60 \mu \mathrm{m}$ in diameter. These features were in identical arrays that were separated by several millimeters. Following patterning, the wafer was diced into small pieces using a Micro Automation Model 1100 Micro Dicing Saw to create multiple samples out of a single lithographic patterning process.

After the substrate was patterned, a $15 \mu \mathrm{l}$ drop of PM suspended in ddi water, having a concentration of $1 \mathrm{mg} / \mathrm{ml}$, was placed over a patterned region. Using gold coated silicon as the negative electrode and the gold layer of the patterned substrate as the positive electrode, 3.3 volts was applied for 60 seconds to achieve PM film deposition. The electrode gap was $1.2 \mathrm{~mm}$. Optical microscope images of the resulting deposition can be seen in Figure 30.
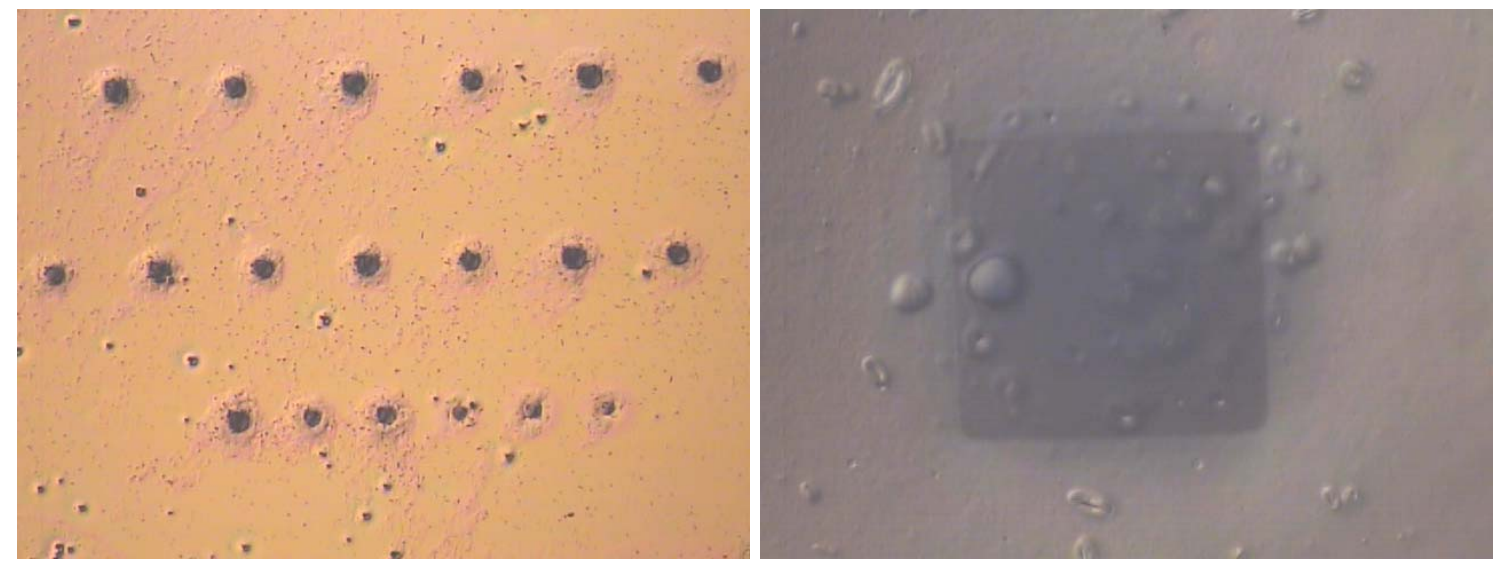

Figure 30: Optical microscope images (left $=5 \mathrm{X}$, right $=100 \mathrm{X})$ of oriented PM films electrodeposited onto a gold coated Si wafer prior to photoresist strip.

After the electrodeposition process was complete, the films were allowed to dry for 24 hours at a humidity of $\sim 52 \%$. The substrate was then submerged in acetone and lightly agitated by hand for 45 seconds. After being removed from the acetone, the 
substrate was dried using nitrogen and imaged on an optical microscope. Images of the resulting patterned PM films can be seen in Figure 31.
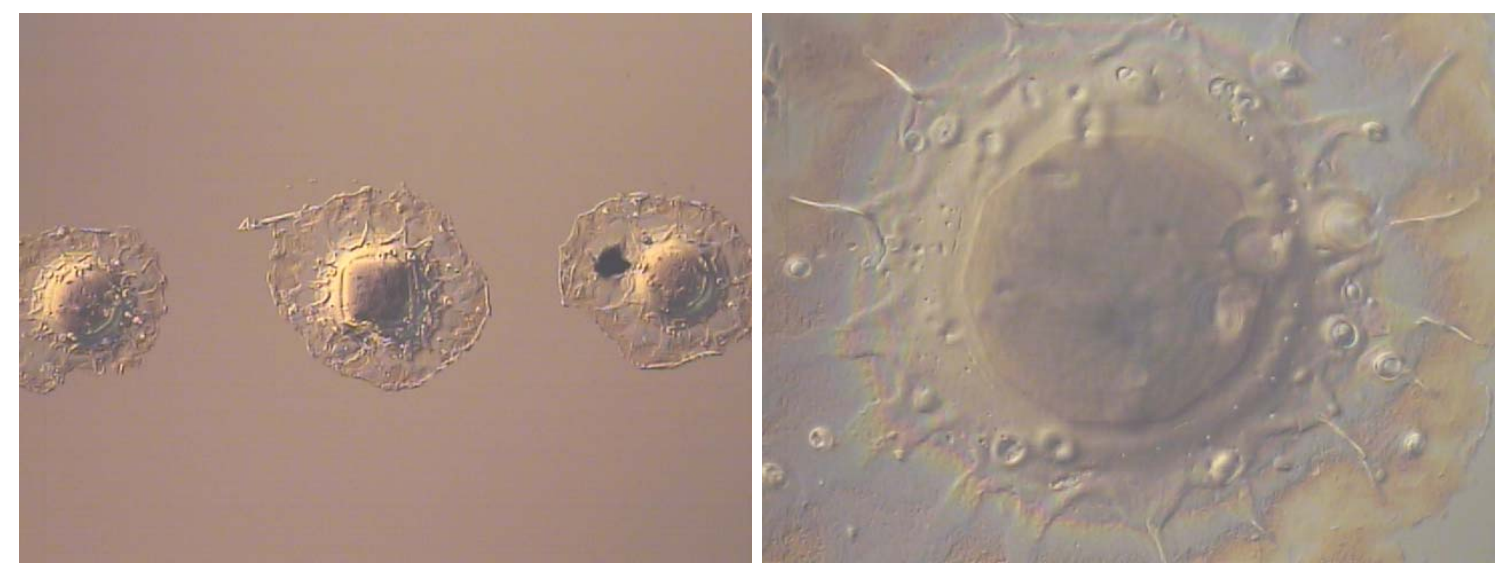

Figure 31: Optical microscope images (left $=20 \mathrm{X}$, right $=100 \mathrm{X})$ of oriented PM films electrodeposited onto a gold coated Si wafer after photoresist strip.

The images of the PM films after the photoresist strip show patterned areas with a radius of material surrounding the patterned areas. To obtain topographical data on both the patterned PM films and radius surrounding each PM film, the sample was sputter coated with $20 \mathrm{~nm}$ of gold and analyzed using an IFM. Two of the resulting IFM scans are shown in Figure 32. 

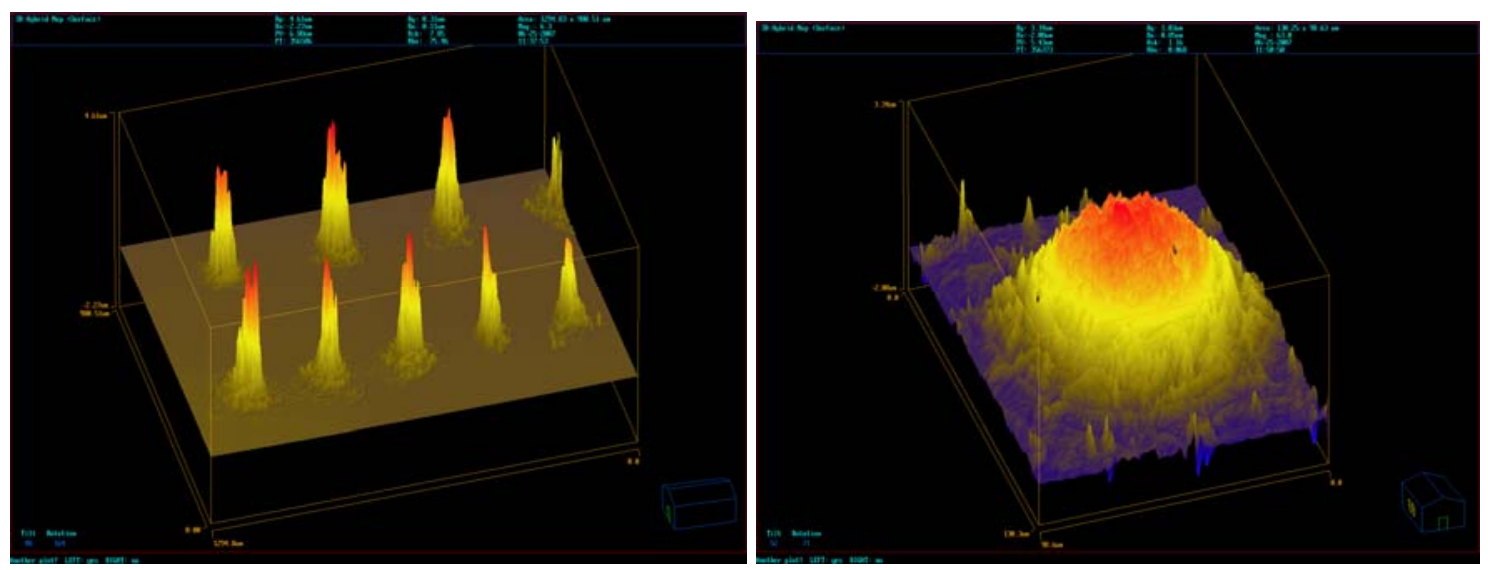

Figure 32: Interferometric microscope analysis (left $=6.2 \mathrm{X}$, right $=63 \mathrm{X}$ ) of oriented PM films electrodeposited onto a gold coated Si wafer after photoresist strip. Average maximum height of patterned regions was $\sim 5.0 \mu \mathrm{m}$.

The IFM analysis showed that the average height of the deposited PM films was 5.0 $\mu \mathrm{m}$. The analysis also showed that the height of the radius surrounding the patterned regions was much less than the height of the patterned PM. It is believed that the radius around the patterned PM regions was photoresist that had not been completely removed by the acetone strip.

To learn more about the radius surrounding the patterned PM films, high magnification images of the films were captured using a FE-SEM. The sample was mounted to the specimen holder using conductive carbon tape. The images in Figure 33 were captured at an accelerating voltage of $10 \mathrm{kV}$, and a working distance of $9 \mathrm{~mm}$. The sample stage was tilted to the maximum of 45 degrees to give some depth perspective to the captured images. 

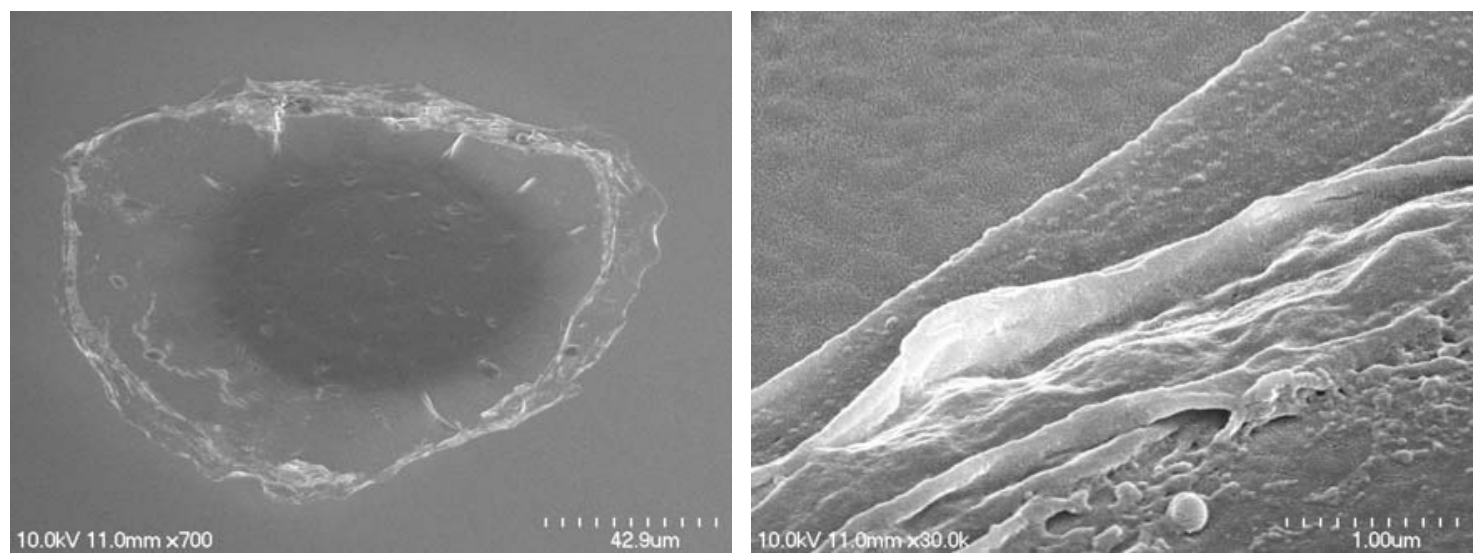

Figure 33: FE-SEM images of a single patterned PM film (left, scale bar $=42.9 \mu \mathrm{m}$ ) and of the transition between the outer rim of the pattern and the substrate (right, scale bar $=1 \mu \mathrm{m}$ ). Accelerating voltage $=10$ $\mathrm{kV}$, working distance $=9 \mathrm{~mm}$, stage tilt $=45$ degrees.

The dark center region of the left image in Figure 33 is the desired PM film pattern. The right image in Figure 33 is a 30,000 X magnification of the transition between the outer rim of the pattern and the gold substrate. The smooth folded features and spherical feature seen in these images lend credibility to the hypothesis that the undesired radii surrounding the PM films are the result of incomplete photoresist removal by the acetone bath. To achieve high quality patterned PM films, an alteration to the photoresist stripping step in the PBBPT was made. The purpose of this alteration was to completely remove the photoresist surrounding the PM films.

\subsubsection{PBBPT Using an Acetone Sonication Bath to Perform Liftoff}

The alteration to the PBBPT process discussed in the previous section was the use of an acetone sonication bath during the photoresist strip / liftoff step of the process. A sonication bath is often employed in thin film photolithography liftoff to speed up the liftoff process and promote a more complete liftoff of unwanted metal films. The sonication helps the acetone reach portions of the photoresist covered by the film. It also helps sections of the film that have been undercut by photoresist removal to break free 
from the surrounding film. Because of the similarities between metal thin film liftoff and the PM film lift off occurring in the PBBPT, assisting PM film liftoff with a sonication bath was a logical next step in developing the PBBPT process.

A PM film was electrodeposited onto a patterned substrate prepared previously (Section 4.1.2). A $25 \mu \mathrm{l}$ drop of suspended PM (4.95 mg/ml) was placed onto the substrate, and gold coated silicon was used to contact the PM suspension. A gap of 1.2 $\mathrm{mm}$ between the top plate and the substrate was maintained, and 4.0 volts was applied for 50 seconds. After deposition, the sample was allowed to dry at 56\% humidity for 24 hours.

To remove the photoresist from the sample and pattern the PM film, the sample was submerged in an acetone sonication bath. A L\&R Ultrasonics Quantrex benchtop sonicator ( $55 \mathrm{Watt}, 43 \mathrm{kHz}$ ) was used to sonicate the sample in acetone for 20 seconds. The sample was removed from the acetone and dried with compressed air. The sample was imaged with an optical microscope and an IFM to evaluate the resulting patterned PM films produced by this process.

Figure 34: Optical microscope images (left $=5 \mathrm{X}$ mag, right $=20 \mathrm{X}$ mag) of patterned $\mathrm{PM}$ films produced by the PBBPT using an acetone sonication bath for film liftoff. Approximate diameter $=60 \mu \mathrm{m}$. 

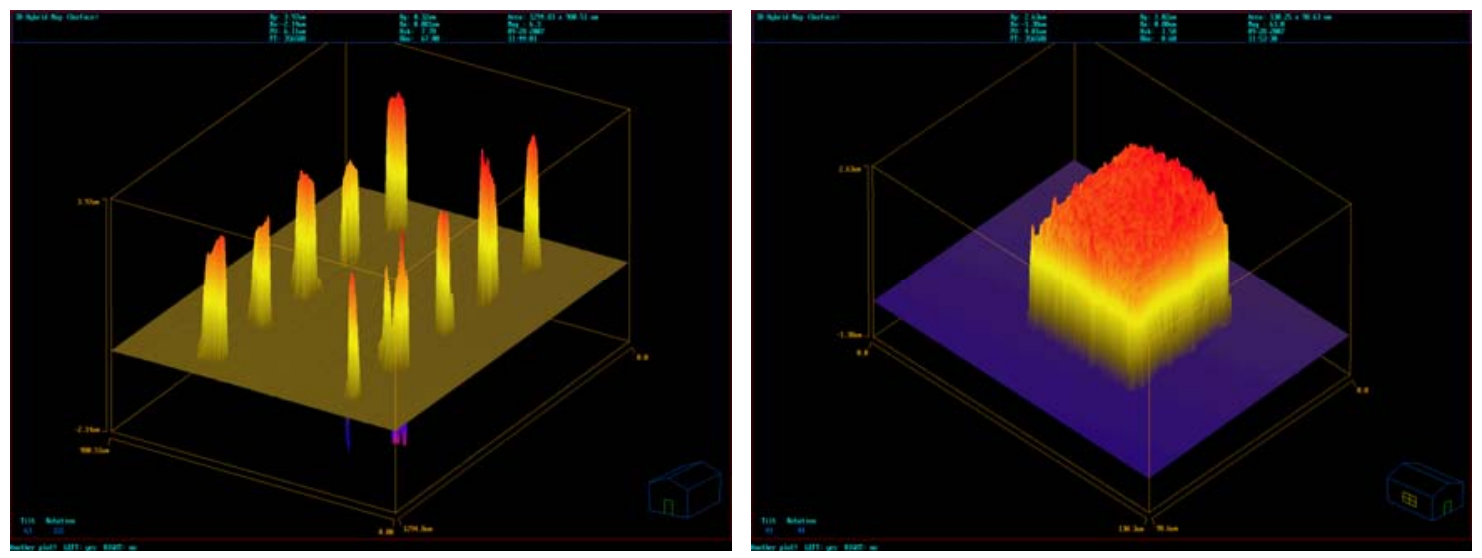

Figure 35: IFM analysis (left $=6.2 \mathrm{X}$ mag, right $=63 \mathrm{X}$ mag) of patterned PM films produced by the PBBPT using an acetone sonication bath for film liftoff. Maximum peak height of the left scan $\sim 4.0 \mu \mathrm{m}$. Feature height of the single square (right) was $\sim 2.6 \mu \mathrm{m}$.

The optical microscope images in Figure 34 show much better patterning results than those achieved without an acetone sonication bath. Irregularities exist at the edges of many of the individual patterned PM films, but the large radius of photoresist surrounding the patterned films is no longer seen. IFM analysis of the sample in Figure 35 also shows much-improved patterning. The areas surrounding the PM films appear smooth, unlike the PBBPT results without the use of an acetone sonication bath. These results were quite encouraging, and prompted further studies to better understand and optimize the PBBPT process using acetone sonication as the photoresist removal technique.

\section{2 $\quad$ PBBPT Process Optimization}

This section describes several process characterization studies performed in order to better understand and optimize the PBBPT process. The first study investigated the effects that changing the PM suspension density during electrodeposition had on the patterned films produced. A second study varied the acetone sonication bath time while holding all other process variables constant. A third study used a photolithography mask 
with squares of decreasing size to determine the minimum feature size that can be created by the PBBPT.

\subsubsection{PM Suspension Density Optimization Study}

Variations in the density of the PM suspension prior to electrodeposition affect the final thickness of the electrodeposited film. By varying the PM suspension density used in the PBBPT, the effect of PM film thickness on the patterning process results was analyzed. To begin this study, eight PM suspensions with varying densities were prepared. The density of each solution was calculated using Beer's law combined with the molecular weight of PM, as discussed in Section 2.3. Optical absorbance measurements were taken using a Nanodrop ND-1000 spectrophotometer. The PM concentration of each sample is shown in Table 2.

Table 2: PM suspension densities calculated using Beer's law and absorbance data from a Nanodrop ND1000 spectrophotometer.

\begin{tabular}{|c|c|}
\hline Sample & PM Density \\
\hline Sample 1 & $4.95 \mathrm{mg} / \mathrm{ml}$ \\
\hline Sample 2 & $2.89 \mathrm{mg} / \mathrm{ml}$ \\
\hline Sample 3 & $1.24 \mathrm{mg} / \mathrm{ml}$ \\
\hline Sample 4 & $0.908 \mathrm{mg} / \mathrm{ml}$ \\
\hline Sample 5 & $0.264 \mathrm{mg} / \mathrm{ml}$ \\
\hline Sample 6 & $0.161 \mathrm{mg} / \mathrm{ml}$ \\
\hline Sample 7 & $0.074 \mathrm{mg} / \mathrm{ml}$ \\
\hline Sample 8 & $0.041 \mathrm{mg} / \mathrm{ml}$ \\
\hline
\end{tabular}

Eight individual substrates from the diced wafer described in Section 4.1.2 were used in this study. Electrodeposition was performed on all eight substrates using the eight PM suspension densities in Table 2. For each of the eight depositions, a $25 \mu \mathrm{l}$ drop of 
suspended PM was placed onto the substrate, and an Au coated Si plate was used to contact the PM suspension. A gap of $1.2 \mathrm{~mm}$ between the top plate and the substrate was maintained, and 4.0 volts was applied for 50 seconds. After deposition, the samples were allowed to dry at 56\% humidity for 24 hours.

An acetone sonication bath was used to remove the photoresist from the substrate, leaving patterned PM films. A sonication time of 20 seconds was used for all eight samples. The acetone sonication bath was performed on each sample individually, and immediately after sonication the samples were dried with compressed air. Following sonication, the samples were imaged with both an optical microscope and an IFM. 


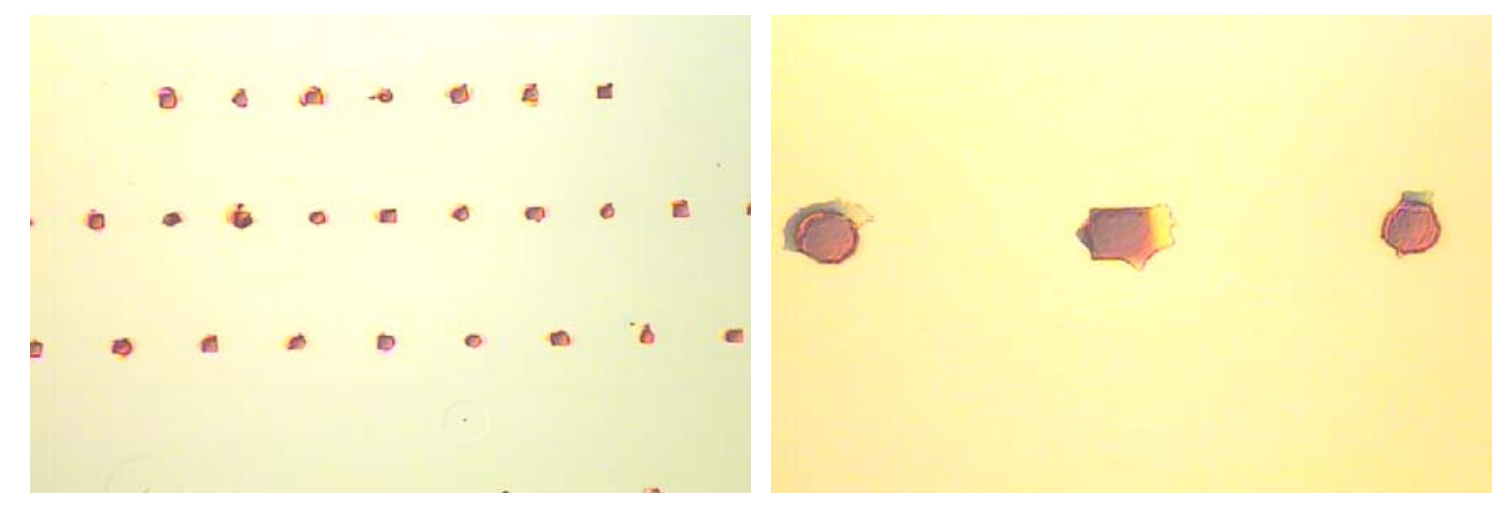

Figure 36: Optical microscope images (left $=5 \mathrm{X}$ mag, right $=20 \mathrm{X}$ mag) of sample $1(4.95 \mathrm{mg} / \mathrm{ml})$.

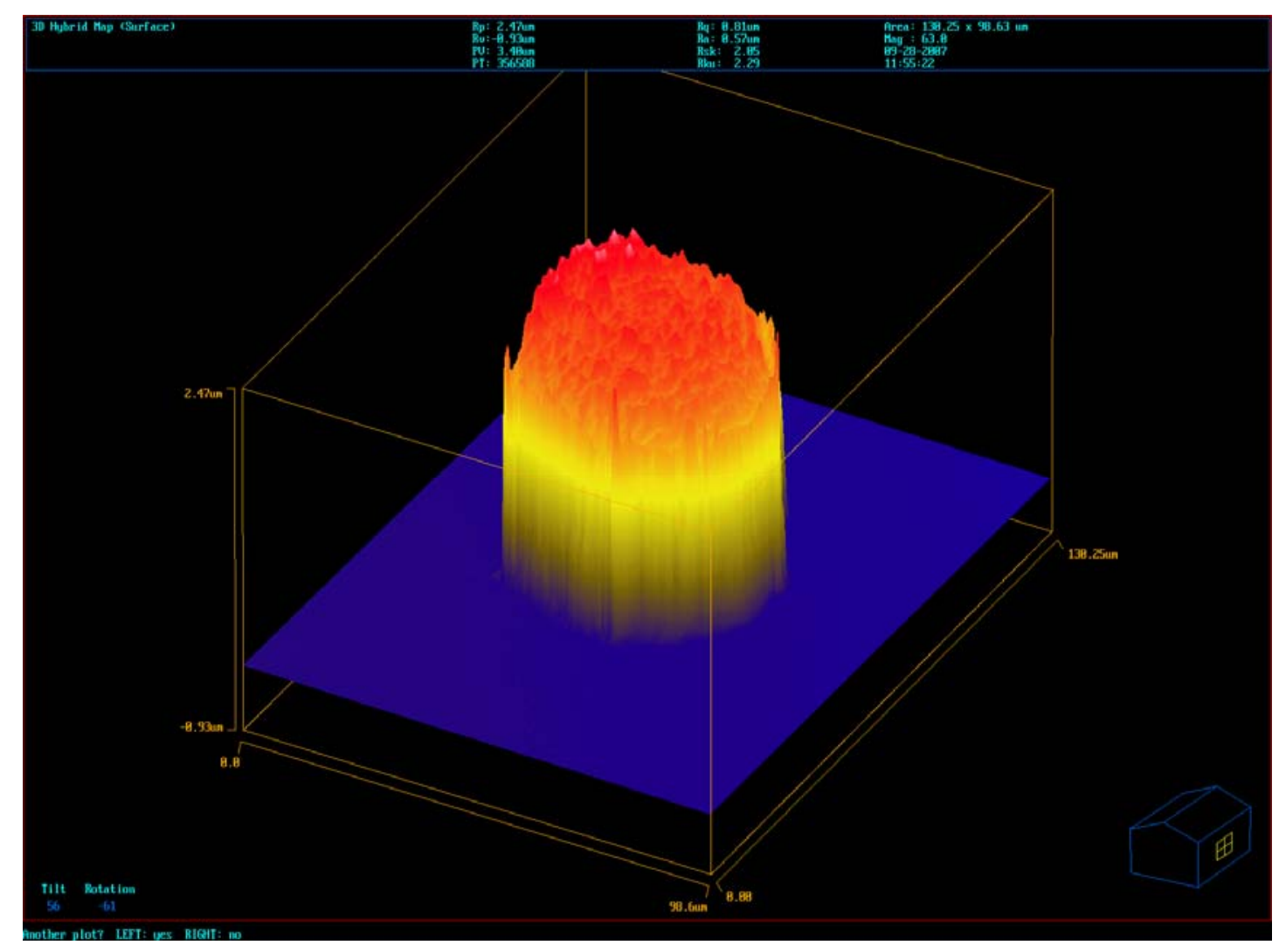

Figure 37: IFM scan (63X mag) of sample $1(4.95 \mathrm{mg} / \mathrm{ml})$. Maximum feature height $\sim 2.6 \mu \mathrm{m}$. 

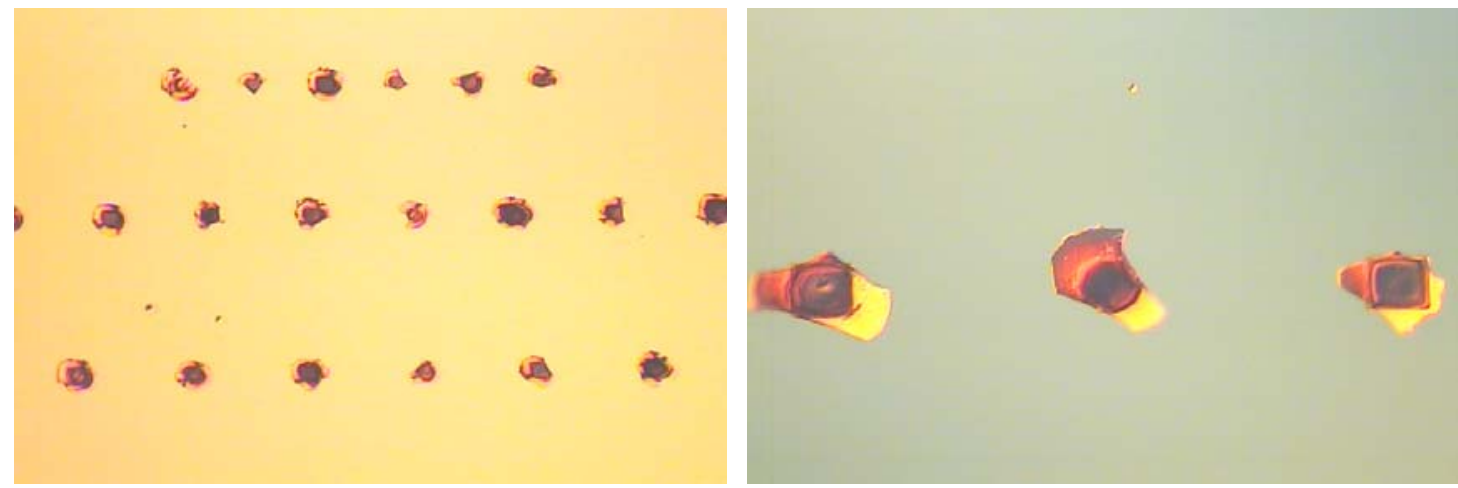

Figure 38: Optical microscope images (left $=5 \mathrm{X}$ mag, right $=20 \mathrm{X}$ mag) of sample $2(2.89 \mathrm{mg} / \mathrm{ml})$.

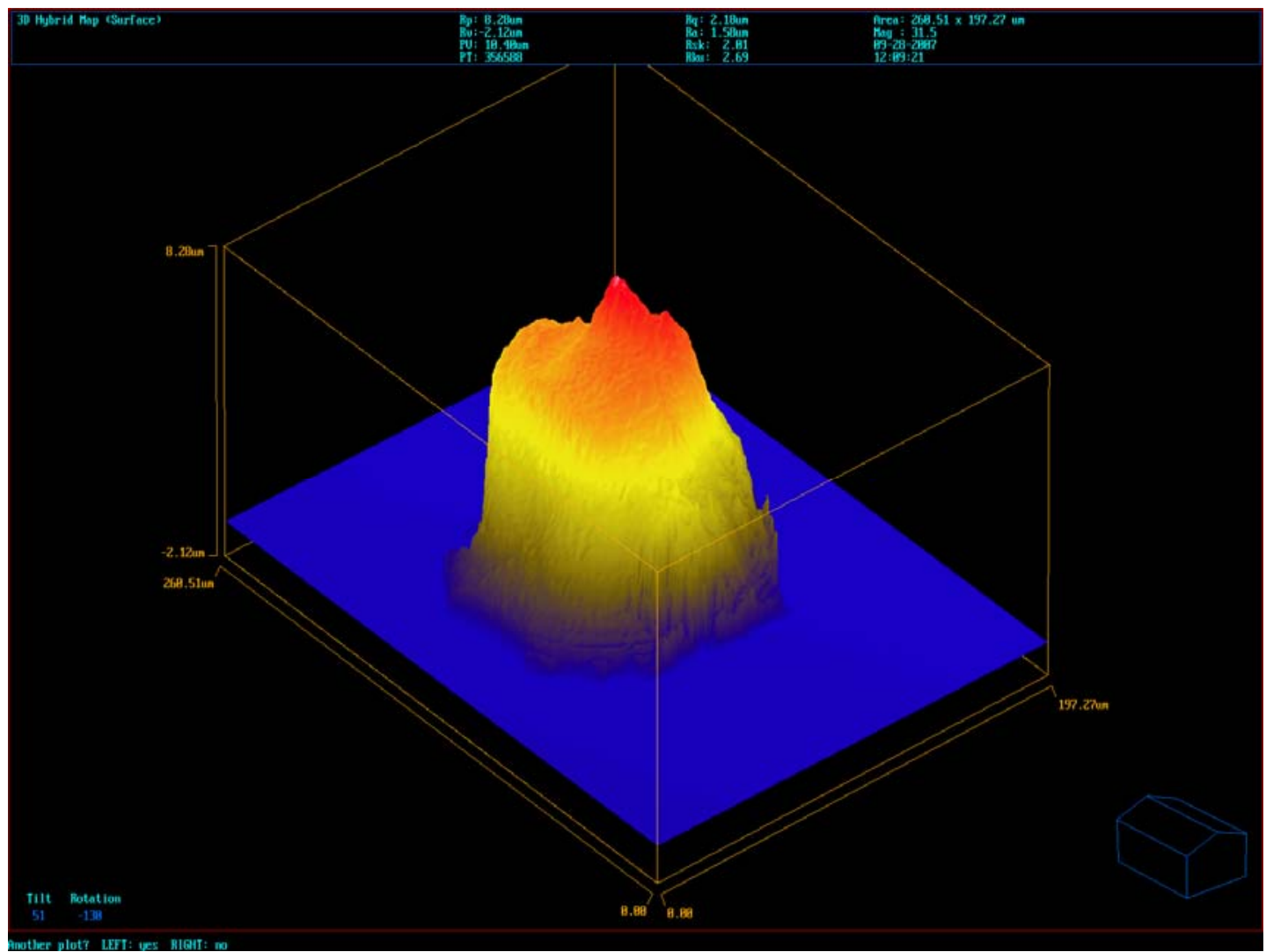

Figure 39: IFM scan (63X mag) of sample $2(2.89 \mathrm{mg} / \mathrm{ml})$. Maximum feature height $\sim 8.3 \mu \mathrm{m}$. 

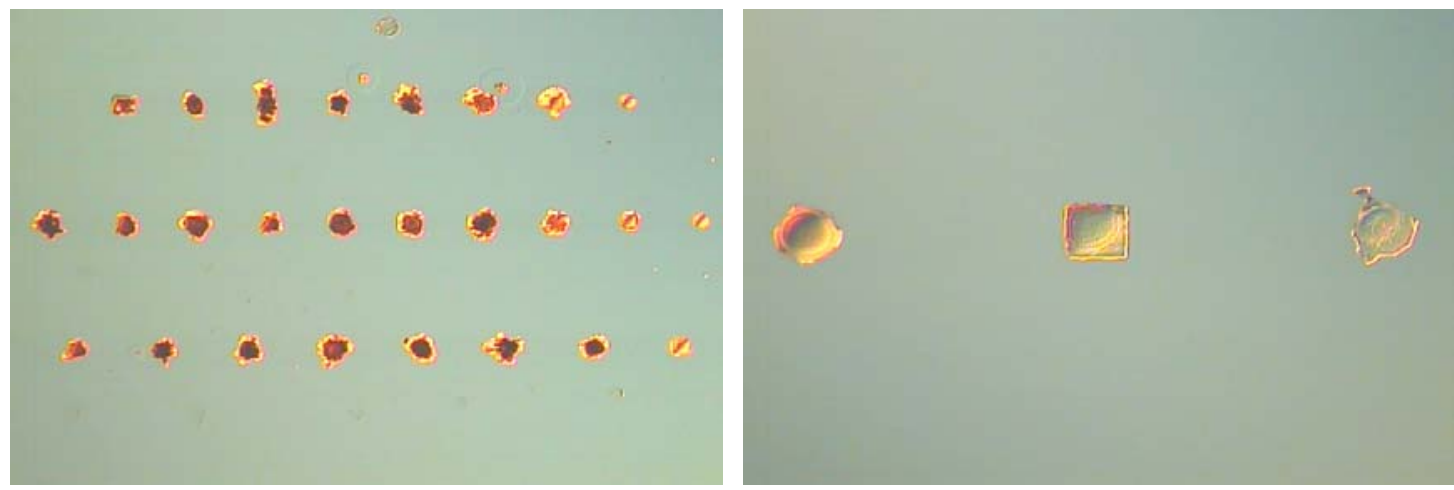

Figure 40: Optical microscope images (left $=5 X$ mag, right $=20 X$ mag) of sample $3(1.24 \mathrm{mg} / \mathrm{ml})$.

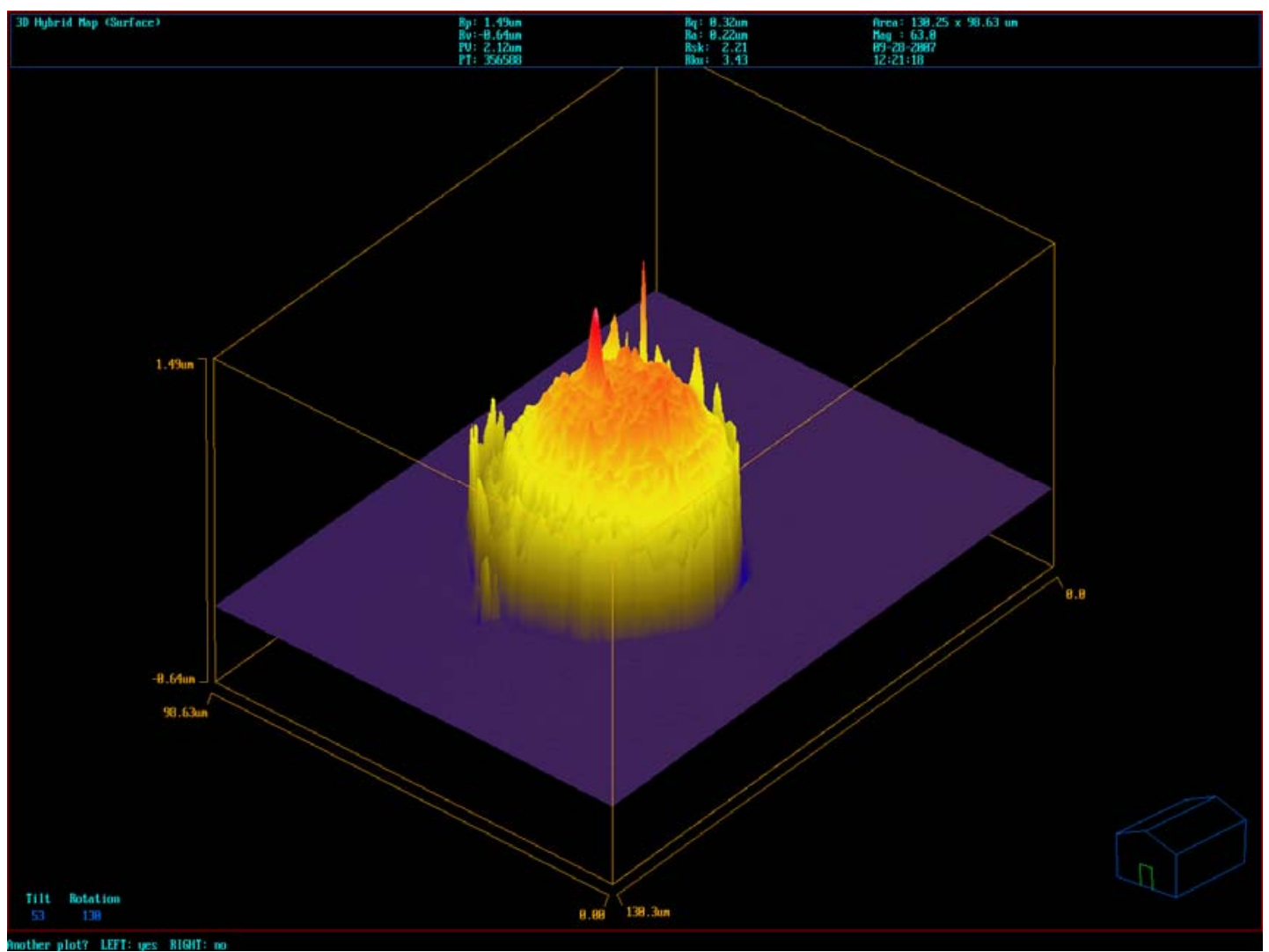

Figure 41: IFM scan (63X mag) of sample $3(1.24 \mathrm{mg} / \mathrm{ml})$. Maximum feature height $~ 1.4 \mu \mathrm{m}$. 

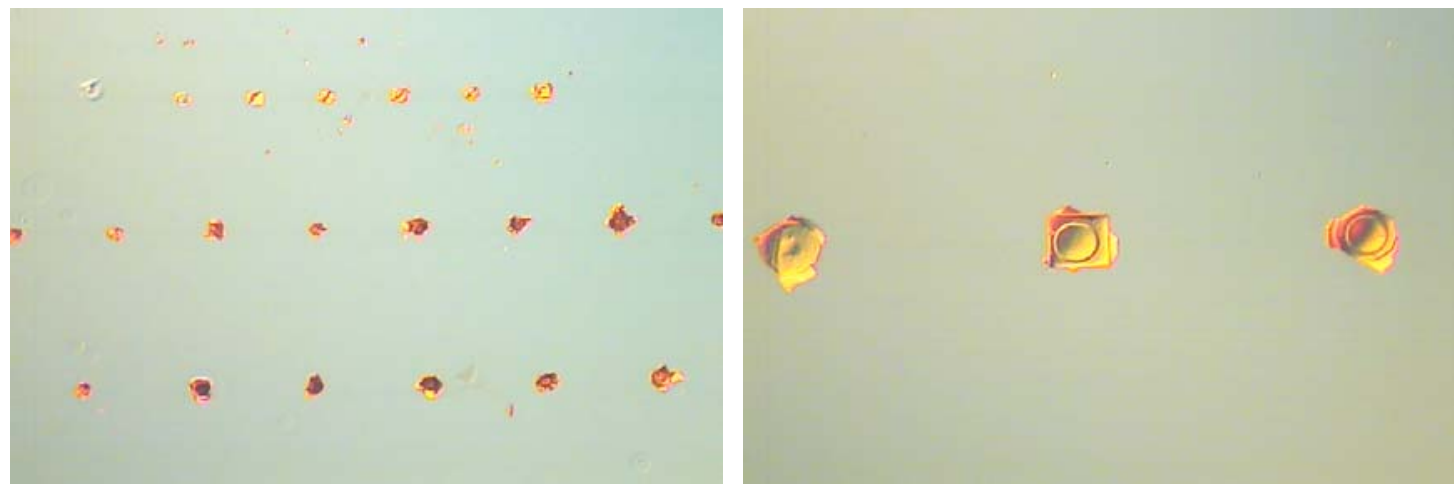

Figure 42: Optical microscope images (left $=5 \mathrm{X}$ mag, right $=20 \mathrm{X}$ mag) of sample $4(0.908 \mathrm{mg} / \mathrm{ml})$.

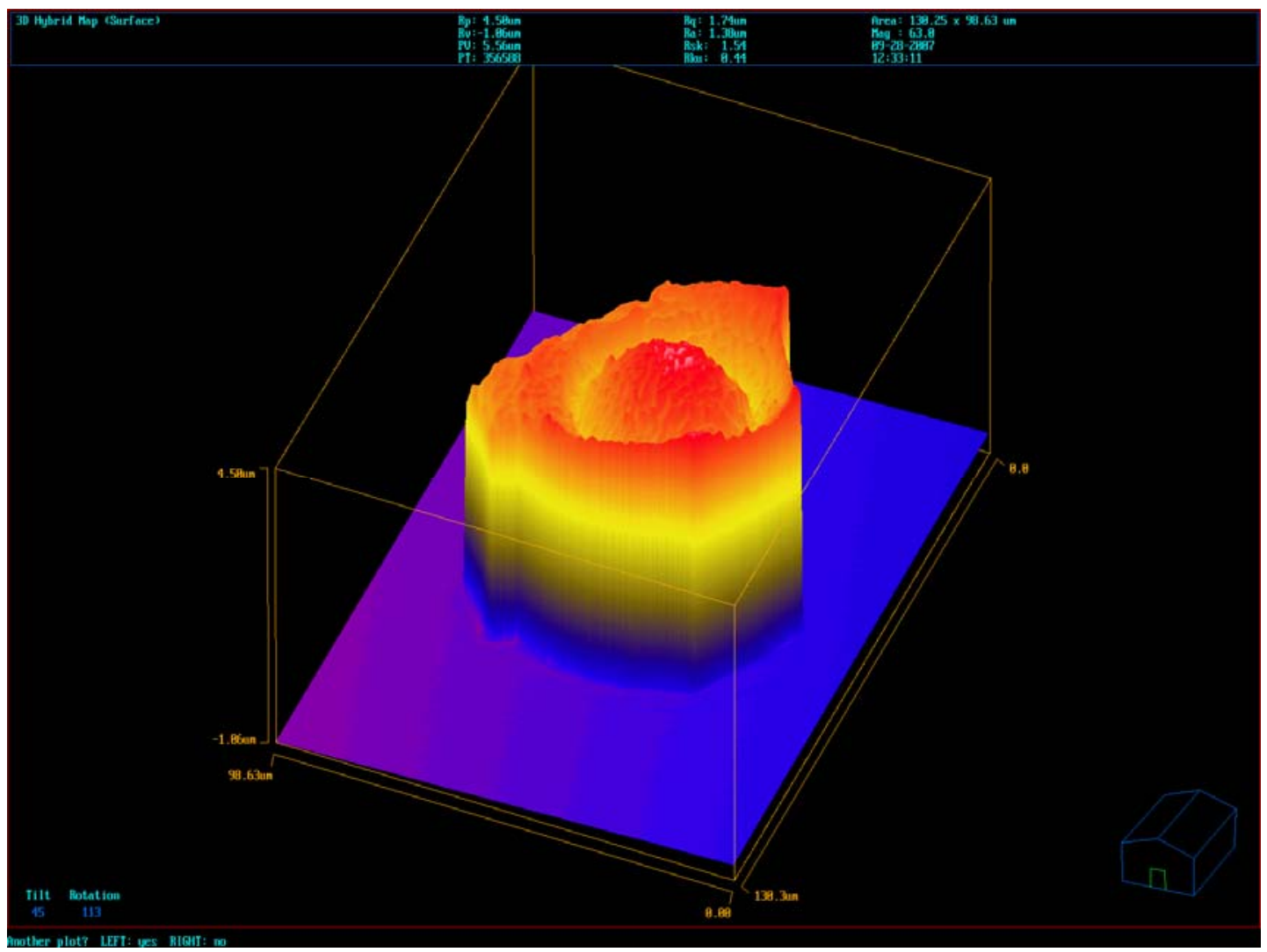

Figure 43: IFM scan (63X mag) of sample 4 (0.908 mg/ml). Maximum feature height $\sim 1.5 \mu \mathrm{m}$. 

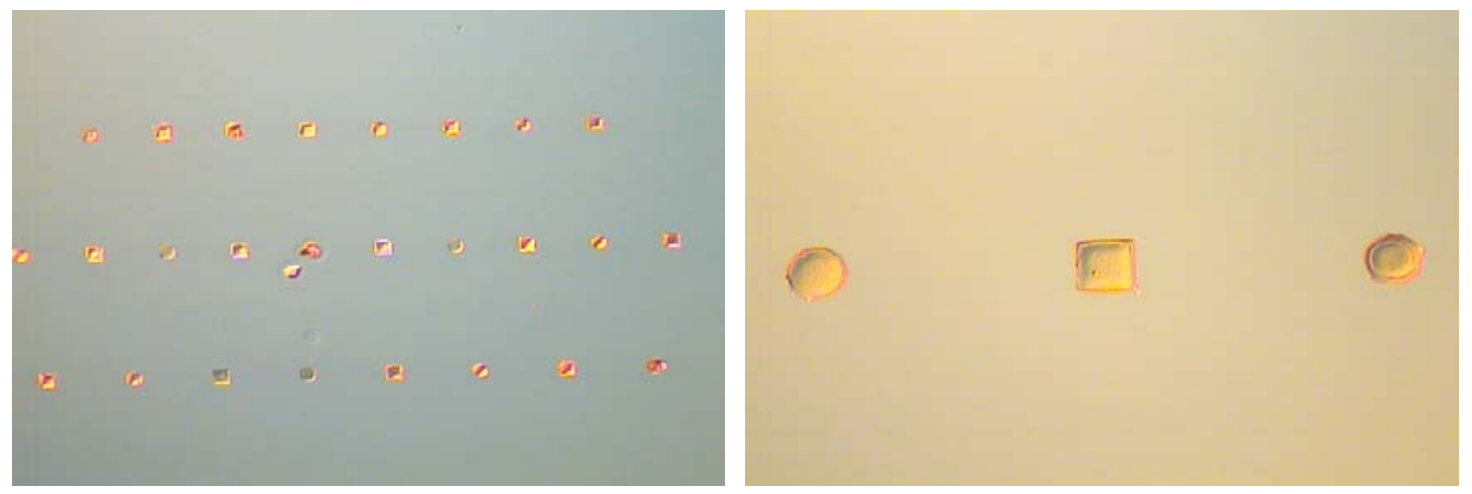

Figure 44: Optical microscope images (left $=5 X$ mag, right $=20 X$ mag) of sample $5(0.264 \mathrm{mg} / \mathrm{ml})$.

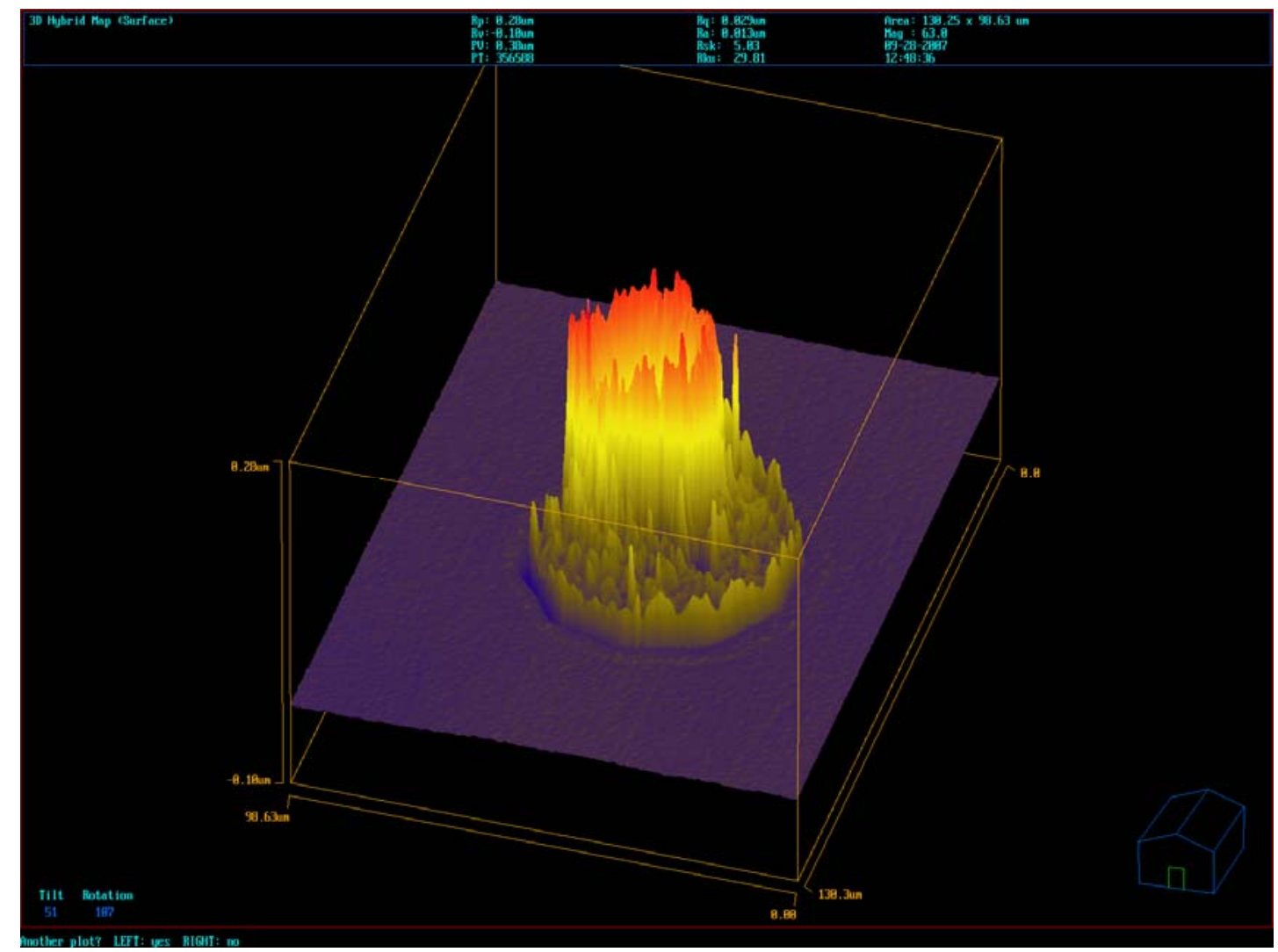

Figure 45: IFM scan (63X mag) of sample $5(0.264 \mathrm{mg} / \mathrm{ml})$. Maximum feature height $\sim 0.28 \mu \mathrm{m}$. 


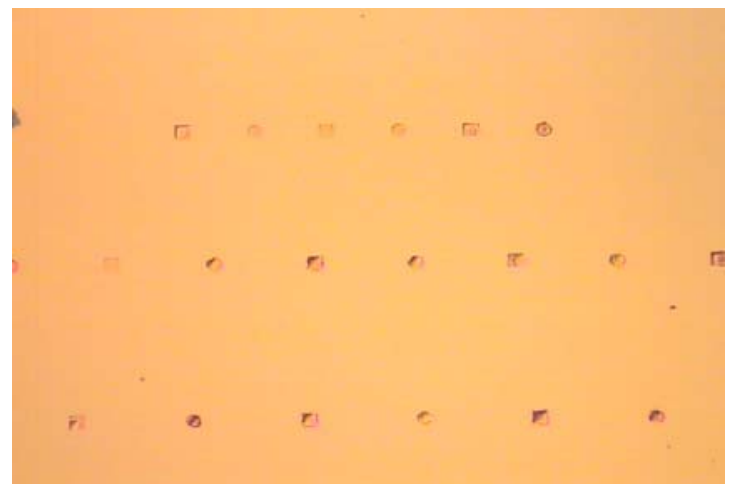

Figure 46: Optical microscope images (left $=5 \mathrm{X}$ mag, right $=20 \mathrm{X}$ mag) of sample $6(0.161 \mathrm{mg} / \mathrm{ml})$.

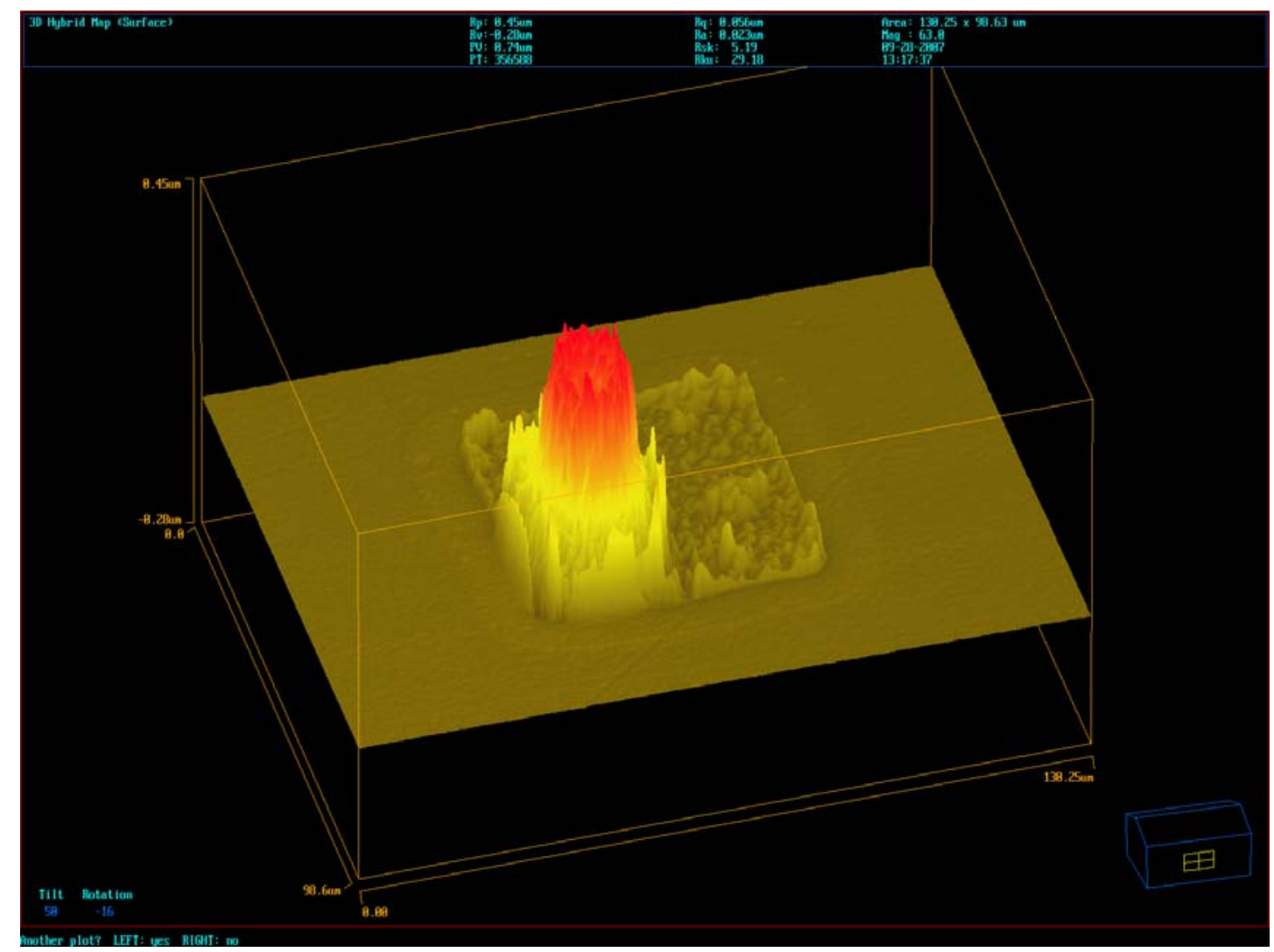

Figure 47: IFM scan (63X mag) of sample $6(0.161 \mathrm{mg} / \mathrm{ml})$. Maximum feature height $\sim 0.45 \mu \mathrm{m}$. 

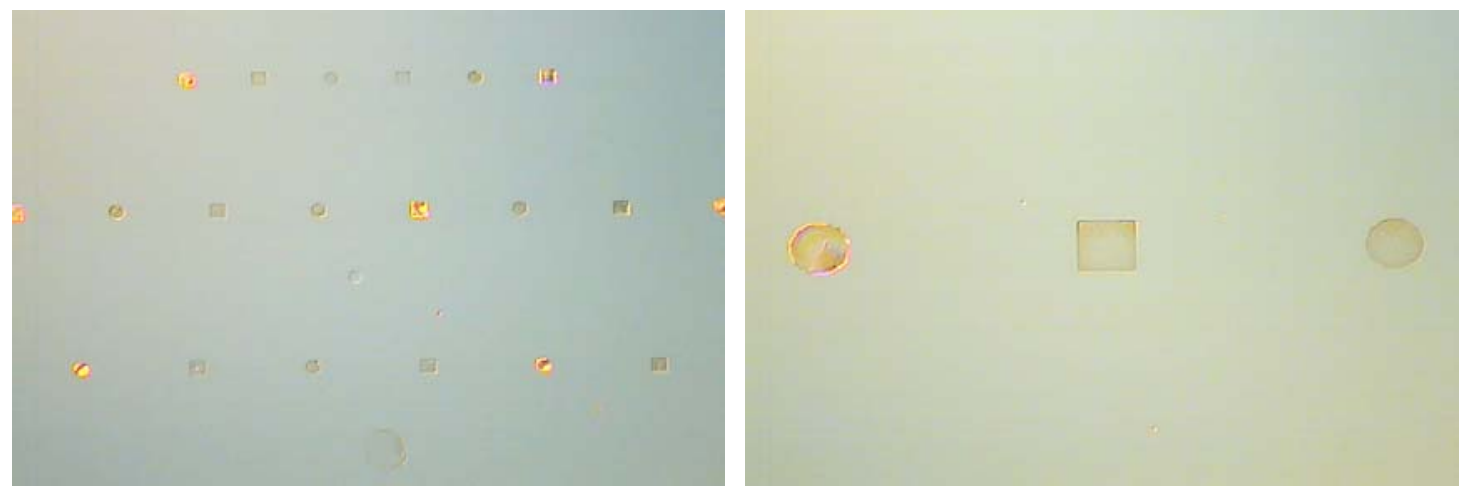

Figure 48: Optical microscope images (left $=5 \mathrm{X}$ mag, right $=20 \mathrm{X}$ mag) of sample $7(0.074 \mathrm{mg} / \mathrm{ml})$.

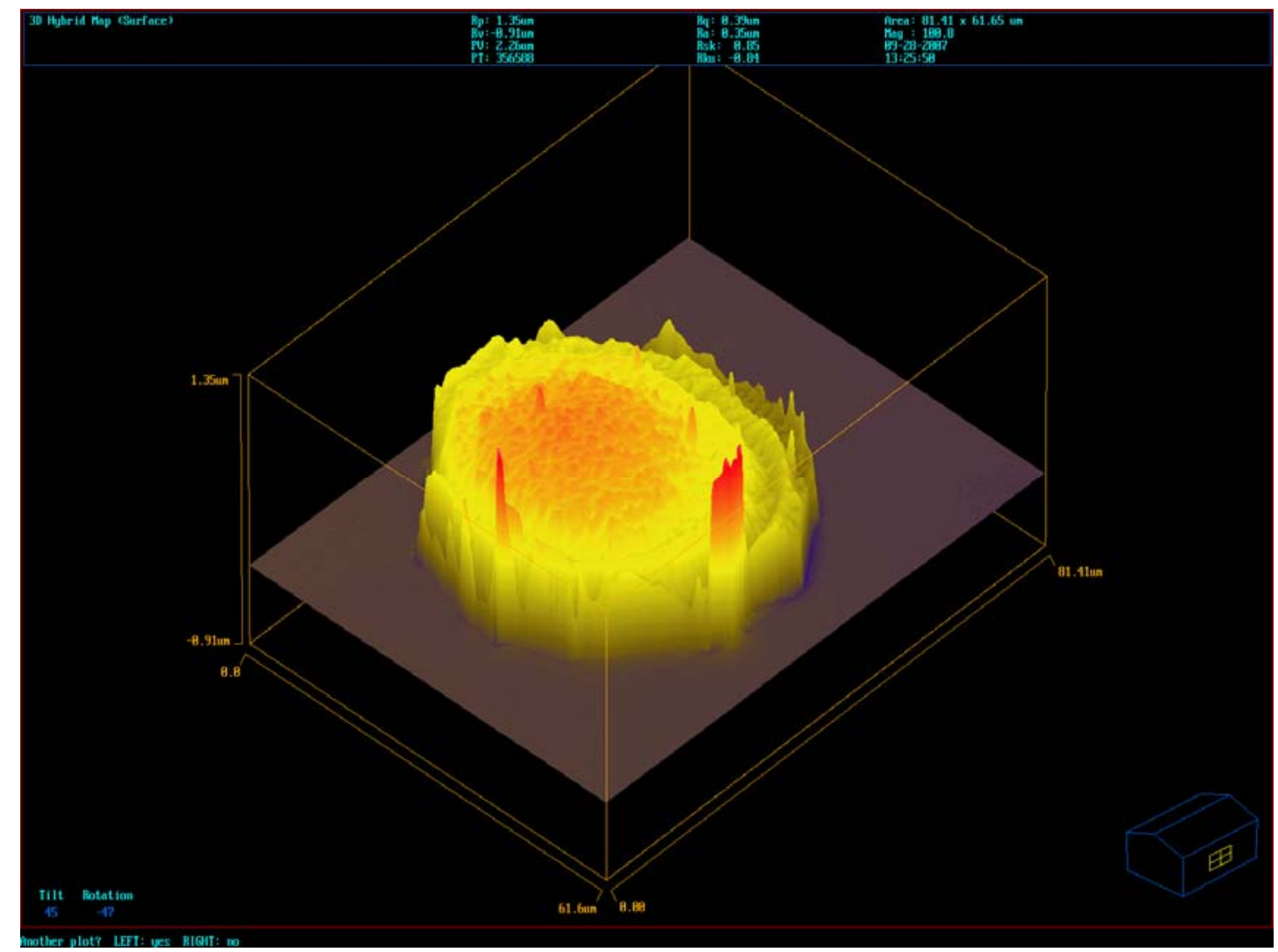

Figure 49: IFM scan (63X mag) of sample $7(0.074 \mathrm{mg} / \mathrm{ml})$. Maximum feature height $~ 1.35 \mu \mathrm{m}$. 

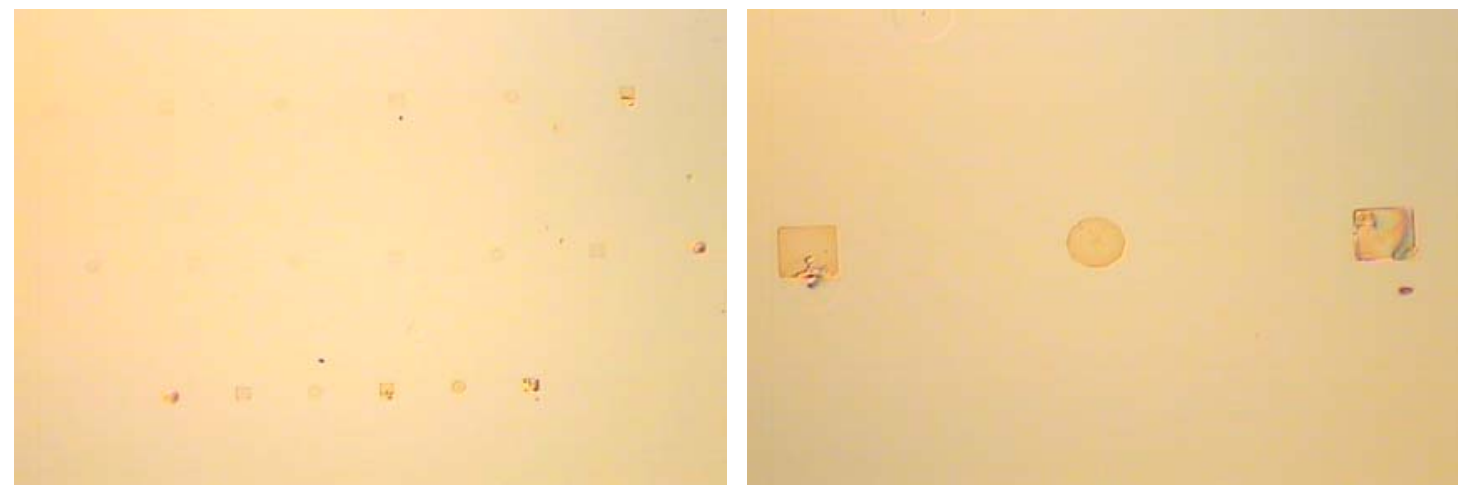

Figure 50: Optical microscope images (left $=5 \mathrm{X}$ mag, right $=20 \mathrm{X}$ mag) of sample $8(0.0041 \mathrm{mg} / \mathrm{ml})$.

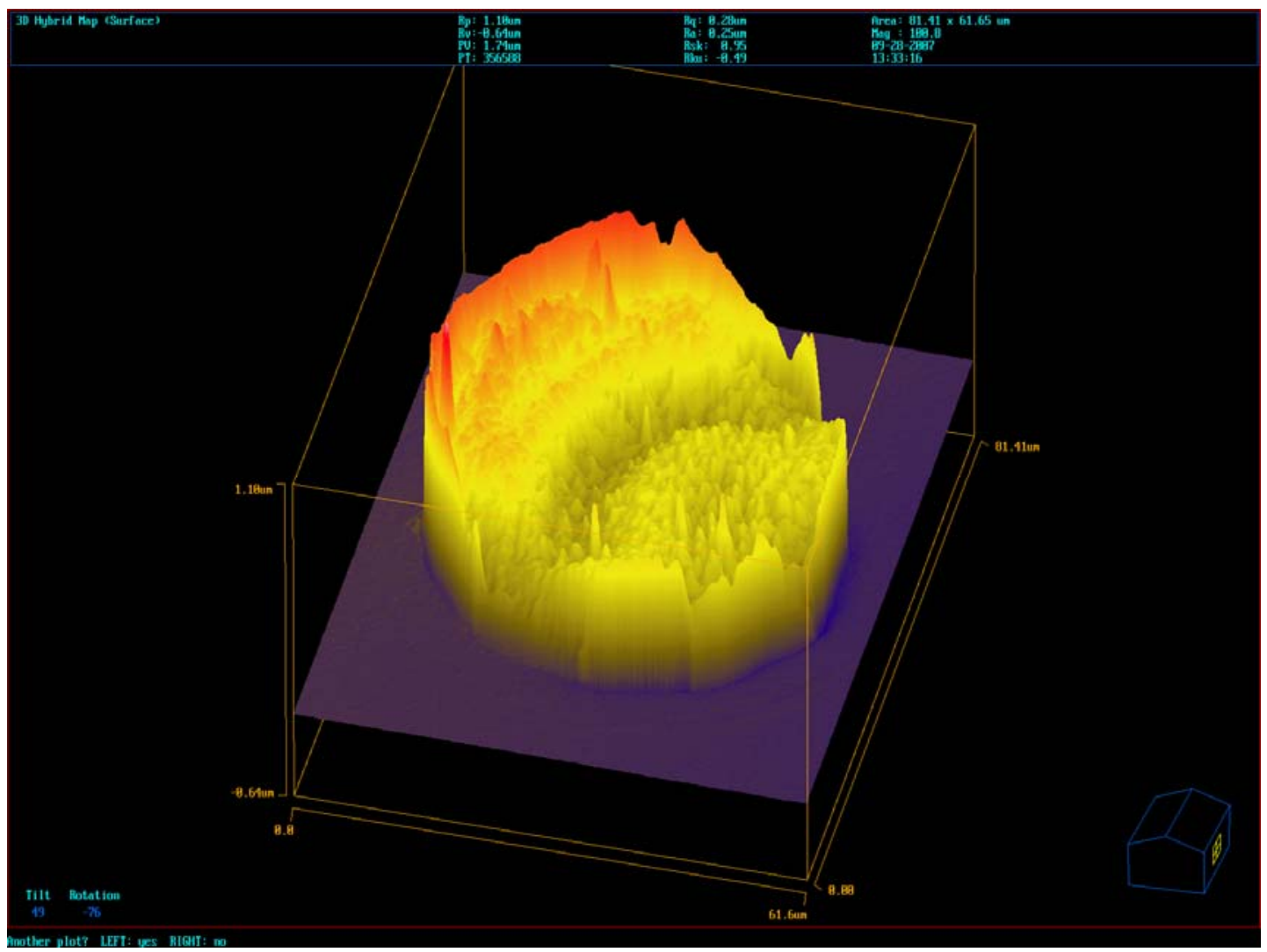

Figure 51: IFM scan (63X mag) of sample $8(0.0041 \mathrm{mg} / \mathrm{ml})$. Maximum feature height $\sim 1.1 \mu \mathrm{m}$.

Figure 36 thru Figure 51 show both optical microscope and IFM analysis of the patterned films produced from all eight PM suspension densities. Samples 1-4 resulted in 
thick PM films that completely covered the desired patterned areas. All four samples show defects around the edges of the patterns. These edge defects may be due to insufficient acetone sonication time. Variations in the height of individual features on all eight samples were also observed. These variations may be due to local variations in the electric field strength during electrodeposition, or a non-uniform distribution of PM patches in the liquid suspension during electrodeposition. Despite these defects, the patterned PM films produced using PM suspensions 1-4 resulted in good pattern quality.

Samples 5-8 produced incomplete or inconsistent PM films over the patterned area. In many cases, thick PM films were only present on a portion of the patterned region. In all four samples some patterned regions showed no remaining PM film. It was also observed that as the PM suspension density decreased, the number of patterned regions not covered by a PM film after sonication increased. These results provided topographical data on the deposition of various densities of PM suspensions onto $\sim 60 \mu \mathrm{m}$ patterned features. As a result of this study, a PM suspension density of $\sim 1.0 \mathrm{mg} / \mathrm{ml}$ or larger was be used during all subsequent experiments to produce quality patterned PM films. The results also demonstrated that further investigation of the acetone sonication process was necessary to limit the edge defects around the patterned PM films.

\subsubsection{Acetone Sonication Bath Time Study}

As demonstrated in the previous study (Section 4.2.1), defects were typically present at the edges of the patterned PM films produced by 20 seconds of sonication. It was believed that these defects may be due in part to incomplete photoresist removal. One way to increase the probability of complete photoresist removal was to increase the sonication time of the samples. To test this hypothesis, an experiment was performed to look at the effect of various acetone sonication bath times on the resulting patterned PM films. Four gold-coated silicon substrates diced from a patterned Si wafer (Section 4.1.2) 
were used as substrates. Electrodeposision was performed to selectively deposit the PM films. A $25 \mu \mathrm{l}$ drop of PM suspension $(\sim 3.0 \mathrm{mg} / \mathrm{ml})$ was placed on each substrate, and a gold coated top electrode was brought into contact with the PM suspension. A voltage of 4.0 volts was applied between the two plates for 50 seconds, and a gap of $1.2 \mathrm{~mm}$ was maintained between the plates. The samples were then allowed to dry at $56 \%$ humidity overnight. After the films had dried, samples 1-4 were placed in an ultrasonic bath containing acetone and sonicated for 30, 45, 60 and 120 seconds respectively. The sonication time was varied to observe the effects of sonicating the samples for longer periods of time. Figures 1-4 show the resulting PM patterned films produced under four different sonication durations. 


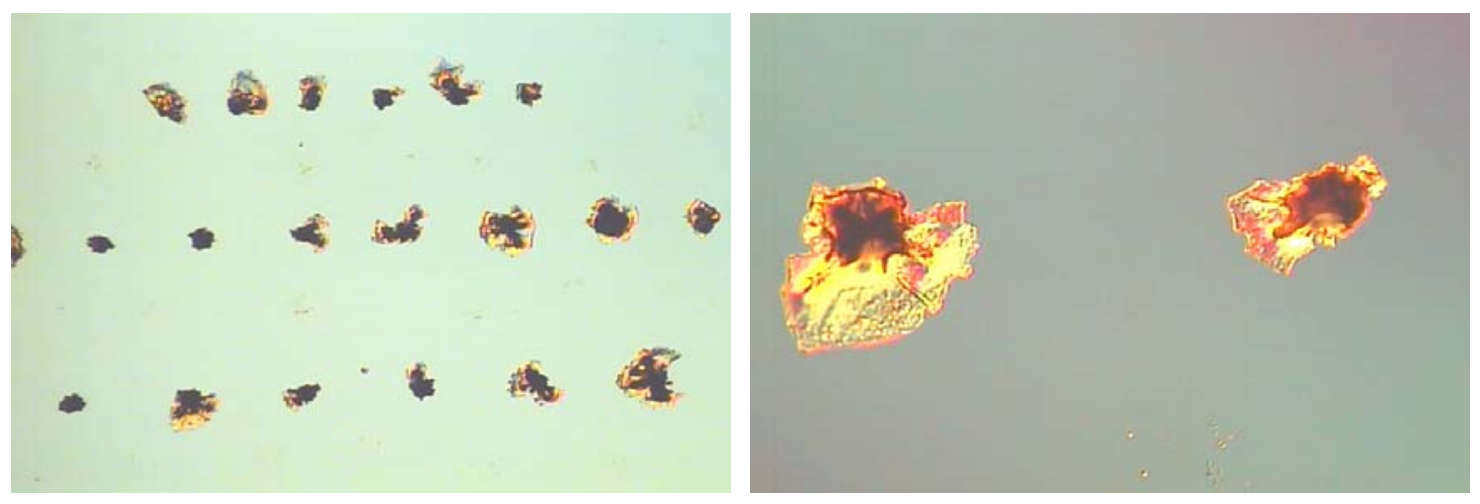

Figure 52: Optical microscope images (left $=5 \mathrm{X}$ mag, right $=20 \mathrm{X}$ mag) of sample 1 (30 sec sonication) .

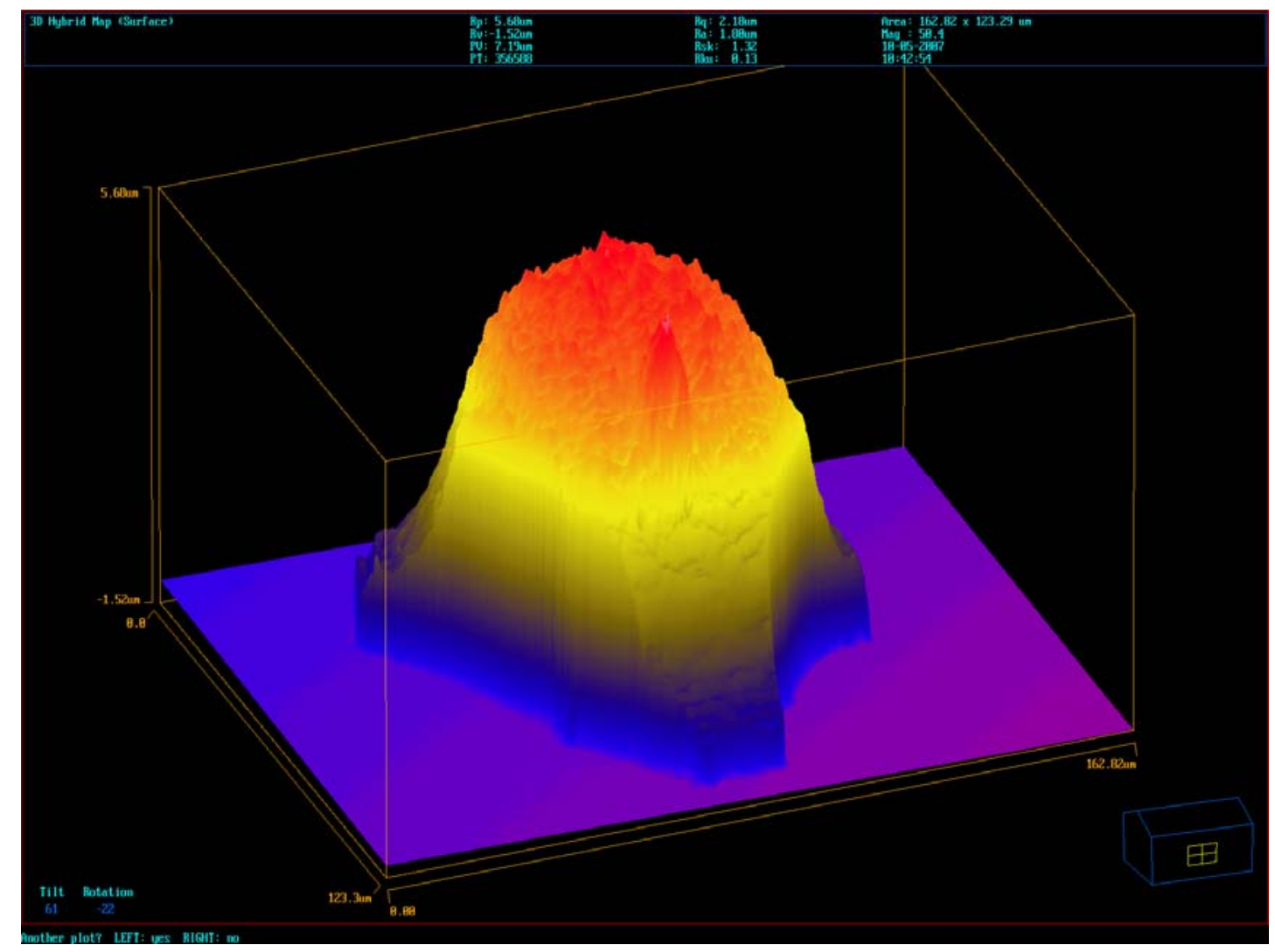

Figure 53: IFM scan (63X mag) of sample 1 (30 sec sonication). Maximum feature height $~ 5.7 \mu \mathrm{m}$. 

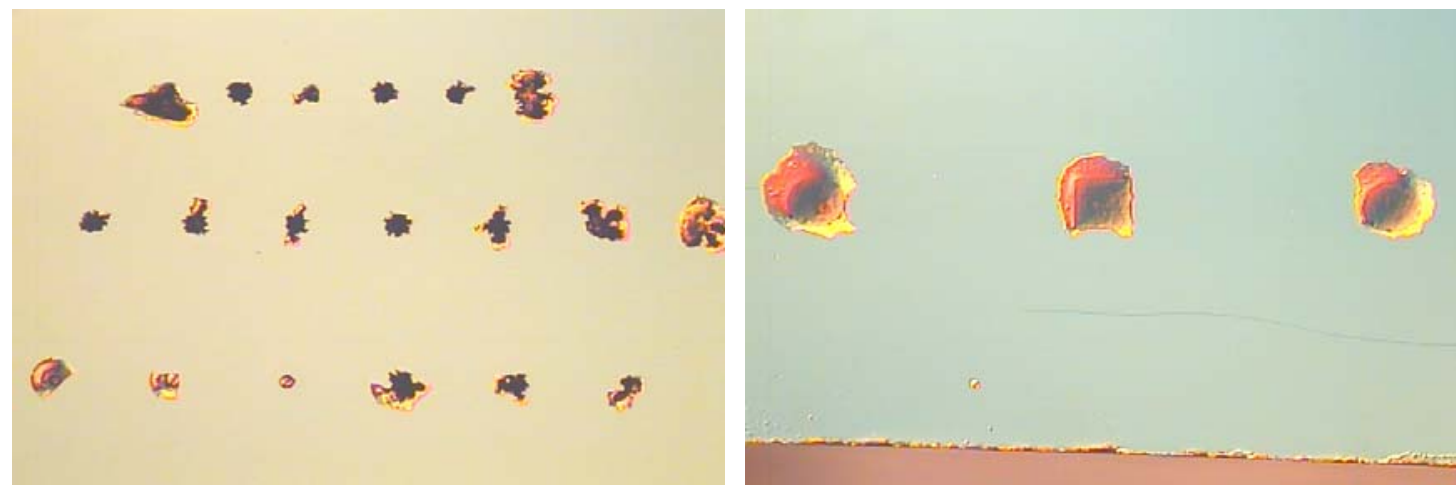

Figure 54: Optical microscope images (left $=5 \mathrm{X}$ mag, right $=20 \mathrm{X}$ mag) of sample 2 (45 sec sonication) .

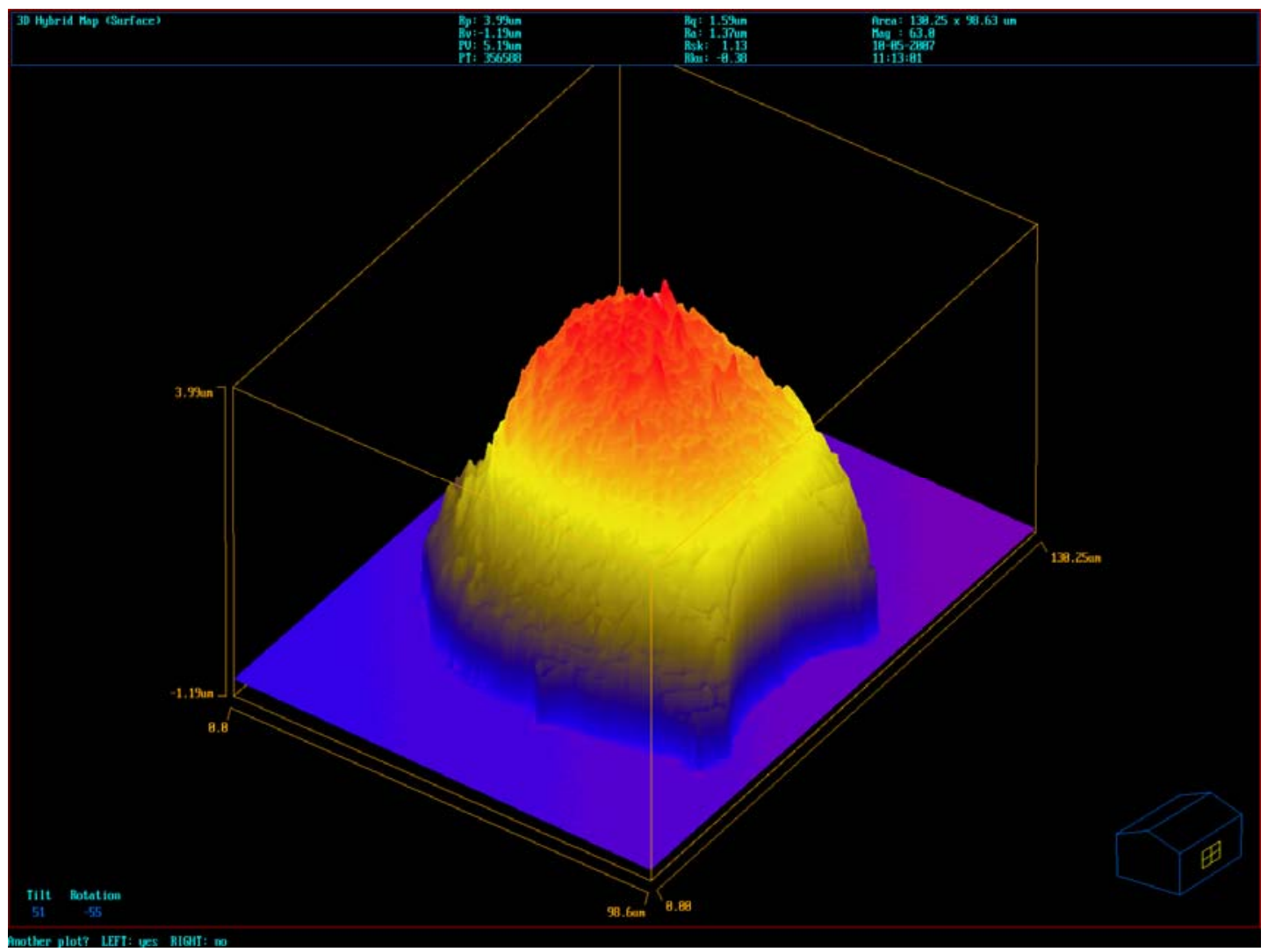

Figure 55: IFM scan (63X mag) of sample 2 (45 sec sonication). Maximum feature height $\sim 4.0 \mu \mathrm{m}$. 

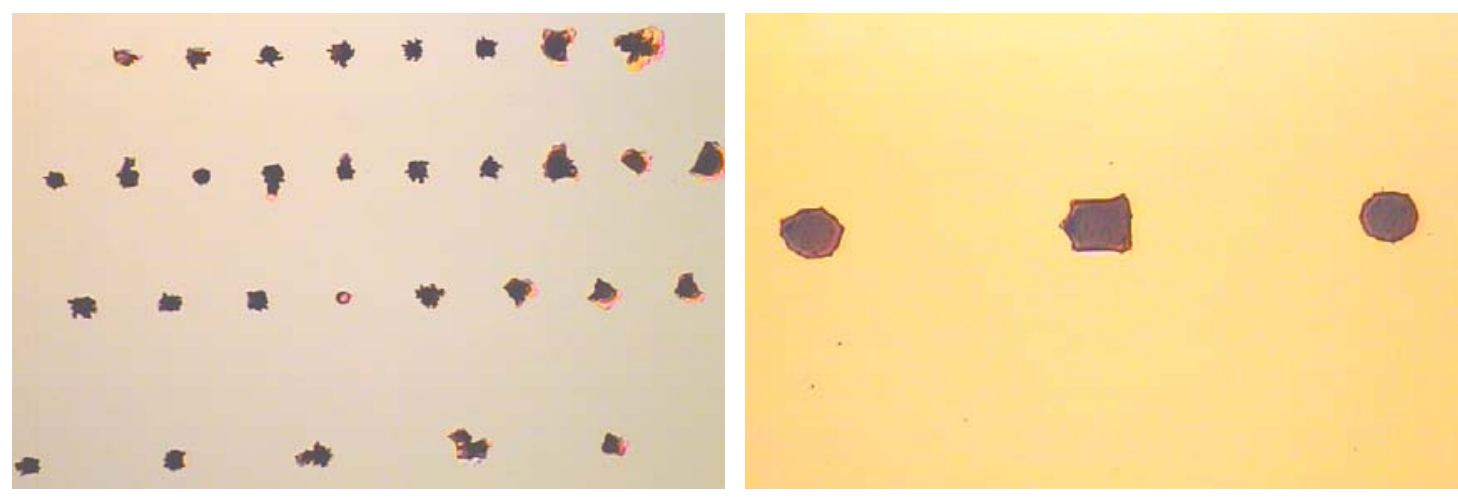

Figure 56: Optical microscope images (left $=5 \mathrm{X}$ mag, right $=20 \mathrm{X}$ mag) of sample 3 (60 sec sonication).

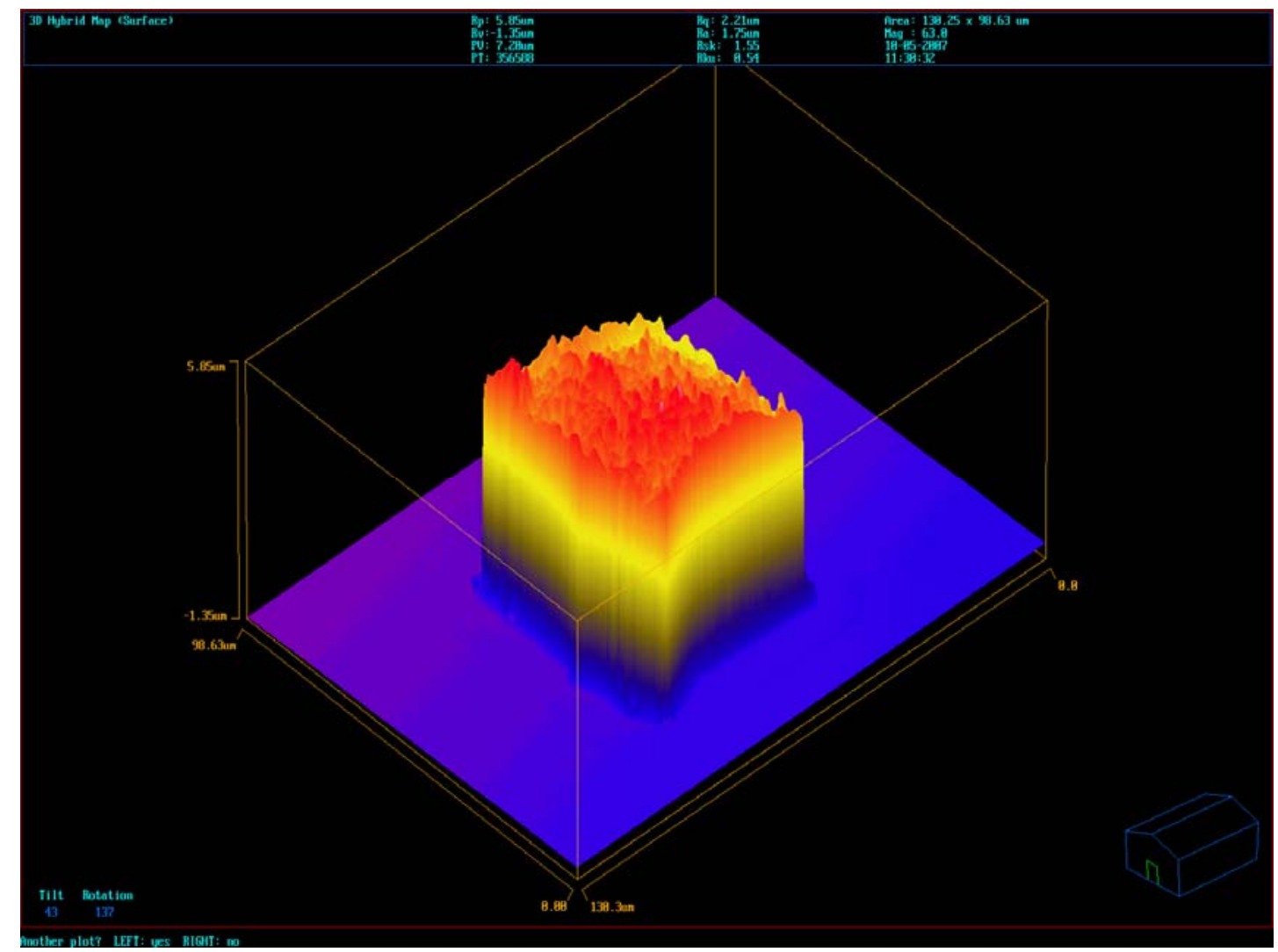

Figure 57: IFM scan (63X mag) of sample 3 (60 sec sonication). Maximum feature height $\sim 5.9 \mu \mathrm{m}$. 

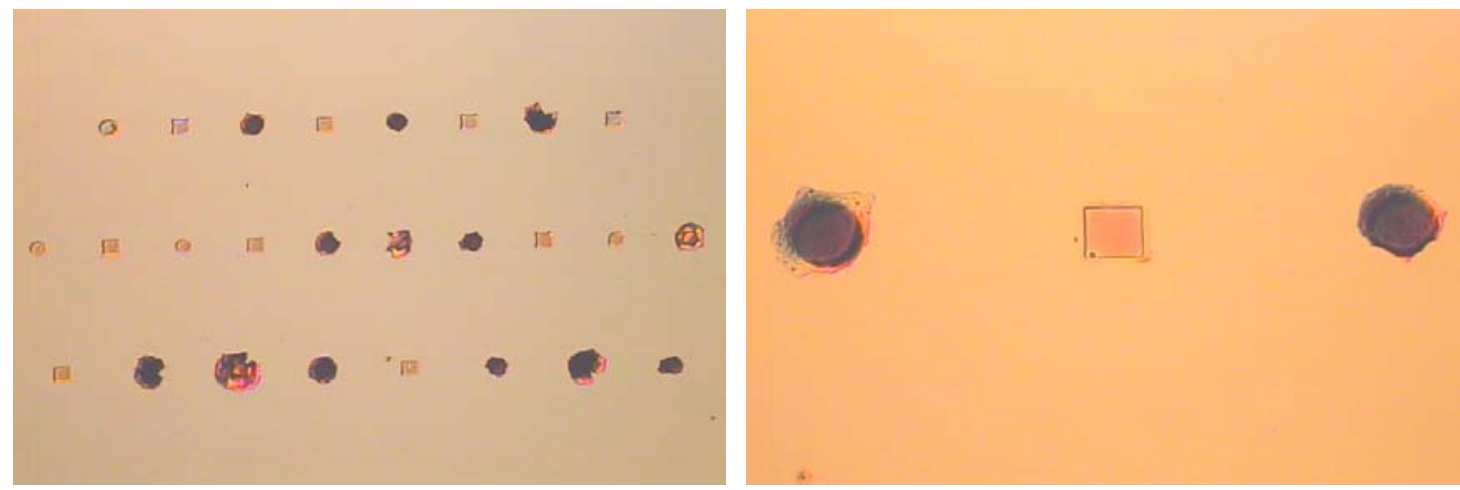

Figure 58: Optical microscope images (left $=5 \mathrm{X}$ mag, right $=20 \mathrm{X}$ mag) of sample 4 (120 sec sonication).

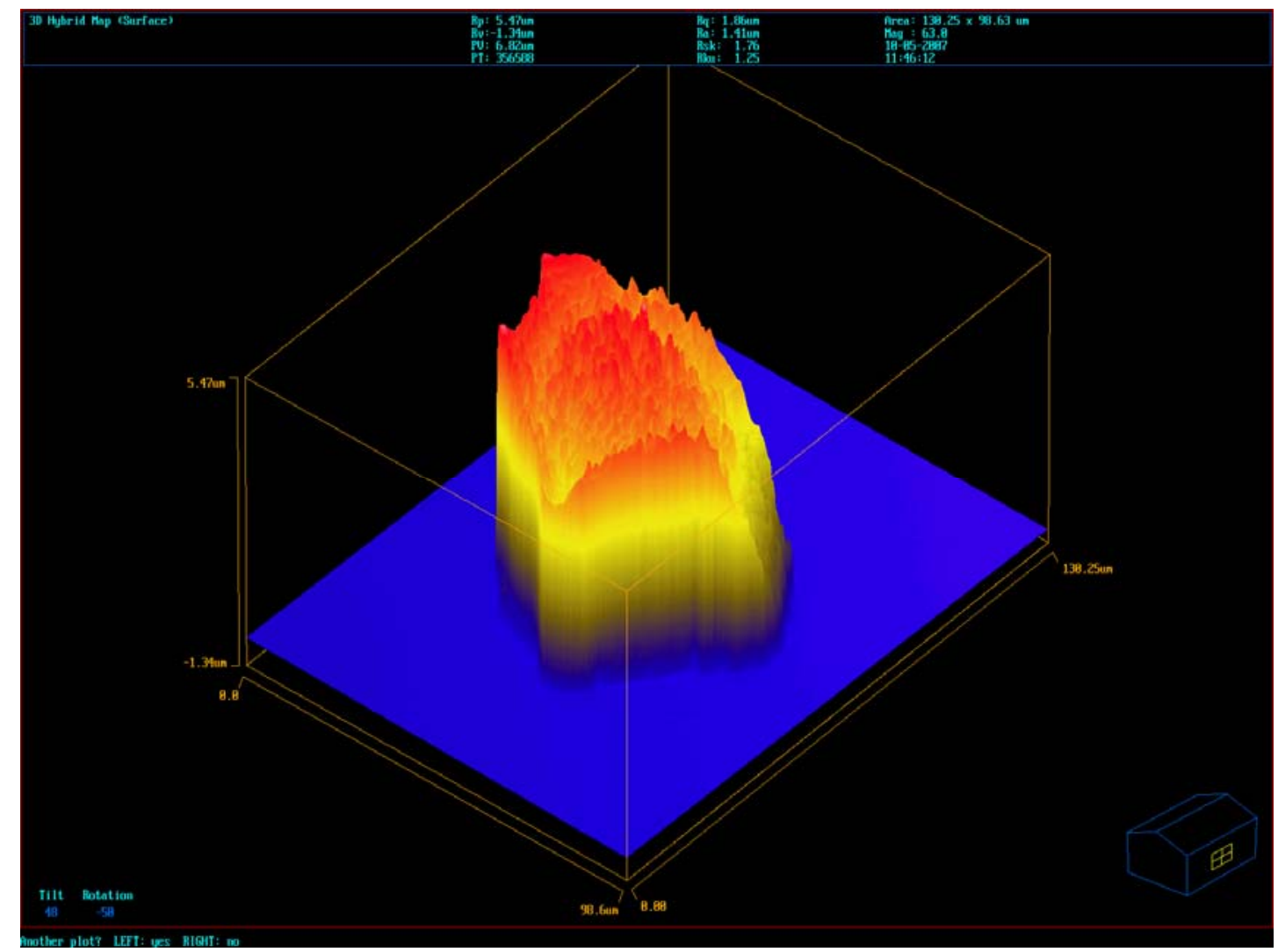

Figure 59: IFM scan (63X mag) of sample 4 (120 sec sonication). Maximum feature height $~ 5.5 \mu \mathrm{m}$.

Based on the optical microscope images and IFM analysis, increasing the sonication time does have an impact on the resulting PBBPT patterns achieved. These results 
indicate that for the above pattern, 60 seconds of acetone sonication to facilitate PM liftoff is optimal. There is a definite decrease in the radius surrounding each of the individual patterns as the sonication time is increased from 30 to 60 seconds. It appears that 120 seconds of sonication time was too much, as many of the PM patterns were removed from the substrate. IFM analysis of these samples indicated that the light colored patterns seen in Figure 58 have virtually no height, indicating that the PM film had been removed. The thickness of the PM patterns that were not removed by sonication ranged from 4-10 $\mu \mathrm{m}$ in size, and sonication time did not appear to play a significant role in the height of the features. It should be noted that the IFM scans shown for each sample represent the highest quality pattern produced on the substrate. The pattern quality shown in Figure 57 was representative of all of the patterns produced on sample 3. Figure 53, Figure 55, and Figure 59 represent the best pattern present on each sample. Samples 1 and 2 showed excess PM and or photoresist surrounding the patterned regions of many of the individual PM patterned films. Sample 4 showed good pattern quality where PM films were present. However, as can be seen in Figure 58, many of the PM deposition sites did not contain PM film after sonication.

This study showed the effectiveness of using a sonicating bath along with acetone to more completely remove photoresist in the PBBPT process, and thus produce better patterns. Without the use of a sonication bath to assist in the removal of photoresist by acetone, only poor quality PM patterns are attainable. Under this set of experimental conditions, 60 seconds of sonication time was found to be optimal. Unfortunately, further research has shown that the optimal sonication time to complete the liftoff process in the PBBPT varies depending on the size and shape of the pattern being produced. Because of this, a visual inspection of each patterned PM sample was performed using optical microscopy to determine if an appropriate amount of acetone sonication was used. 


\subsubsection{PBBPT Minimum Feature Size Study}

One important characteristic of any patterning process is the minimum feature size achievable using the process. To determine this characteristic, a mask was designed using Cadence, and fabricated by Bandwidth Foundry. The chrome glass mask contained 18 rows of squares. Each row was seperated from the next by $400 \mu \mathrm{m}$. The size and spacing of the squares in each row of the mask are shown in Table 3.

Table 3: Size and spacing of squares contained in each row of the PBBPT minimum feature size mask.

\begin{tabular}{|c|c|c|}
\hline Row \# & $\begin{array}{c}\text { Square Size } \\
(\boldsymbol{\mu} \mathbf{m})\end{array}$ & Spacing between squares $(\boldsymbol{\mu m})$ \\
\hline 1 & 60 & 60 \\
\hline 2 & 60 & 180 \\
\hline 3 & 50 & 50 \\
\hline 4 & 50 & 150 \\
\hline 5 & 40 & 40 \\
\hline 6 & 40 & 120 \\
\hline 7 & 30 & 30 \\
\hline 8 & 30 & 90 \\
\hline 9 & 25 & 25 \\
\hline 10 & 25 & 75 \\
\hline 11 & 20 & 20 \\
\hline 12 & 20 & 60 \\
\hline 13 & 15 & 15 \\
\hline 14 & 15 & 45 \\
\hline 15 & 10 & 10 \\
\hline 16 & 10 & 30 \\
\hline 17 & 5 & 5 \\
\hline 18 & 5 & 15 \\
\hline
\end{tabular}

An ITO coated glass substrate was patterned using the mask described above. The photolithography protocol described in Section 4.1.1 was used to pattern the ITO substrate. The aligner was run in hard contact mode to achieve the best possible pattern replication in the photoresist. After the substrate was patterned, the resist was stripped 
from a corner of the substrate not containing any pattern using acetone. This exposed the ITO surface, allowing for electrical contact during the electrodeposition process.

Electrodeposition was performed by placing three $15 \mu \mathrm{l}$ drops of PM suspension $(2.67 \mathrm{mg} / \mathrm{ml})$ onto the substrate. The drops were spaced so that a large single suspension of PM was formed on the substrate, covering the patterned area. Gold coated silicon was brought into contact with the suspension, and rested on spacers that set a separation of 1.2 $\mathrm{mm}$ between the substrate and the top plate. A voltage of 4.0 volts was applied between the substrate and the top plate for 40 seconds. The top plate was removed, and the substrate was submerged one time in ddi water. The sample was allowed to dry under ambient conditions overnight. After the sample was dried, it was coated with $\sim 8 \mathrm{~nm}$ of gold. This was a departure from the initial PBBPT process flow, and is discussed in more detail in Section 4.3.

After the Au deposition, the sample was subjected to an 80 second acetone sonication bath to lift off the unwanted PM and Au films from the substrate. What remained were patterned PM films with an $\sim 8 \mathrm{~nm}$ film of gold on top of the PM. To prepare the sample for imaging, an additional $6 \mathrm{~nm}$ of gold was coated onto the sample using the Hummer 6.2 RF sputtering system. The sample was then imaged using a Hitachi S-4700 FE-SEM. A stage tilt of 45 degrees was used to gain some depth perspective, and was the reason the square patterns shown below appear distorted. 

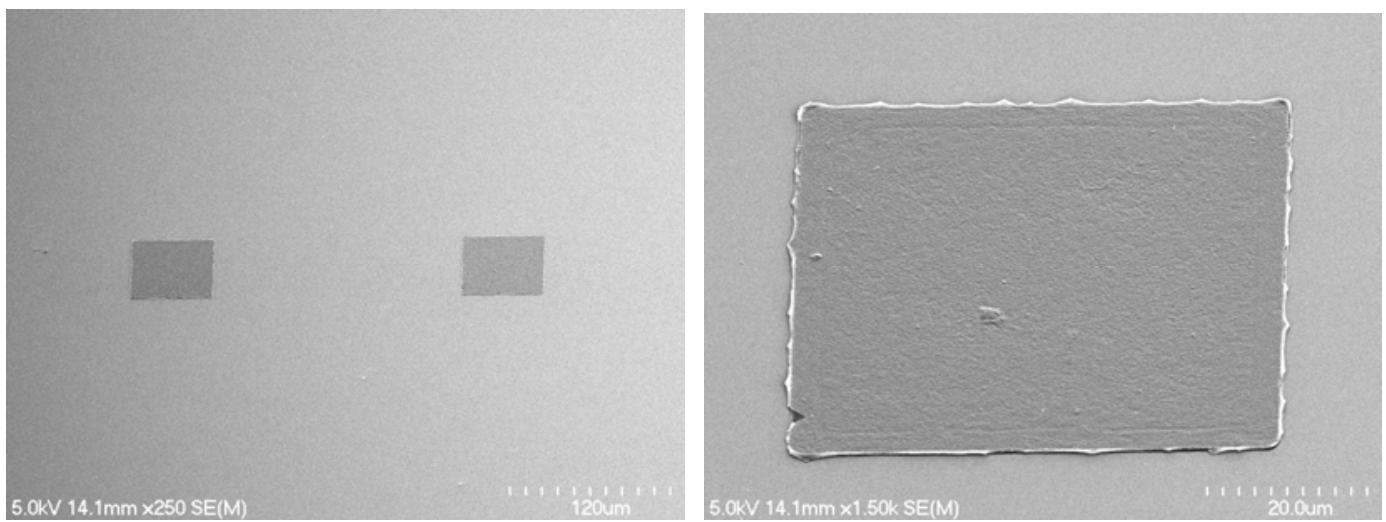

Figure 60: FE-SEM images (left $=120 \mu \mathrm{m}$ scale bar, right $=20 \mu \mathrm{m}$ scale bar) of $60 \mu \mathrm{m}$ square patterned PM films. Accelerating voltage $=5 \mathrm{kV}$, working distance $=12 \mathrm{~mm}$, Stage tilt $=45$ degrees .
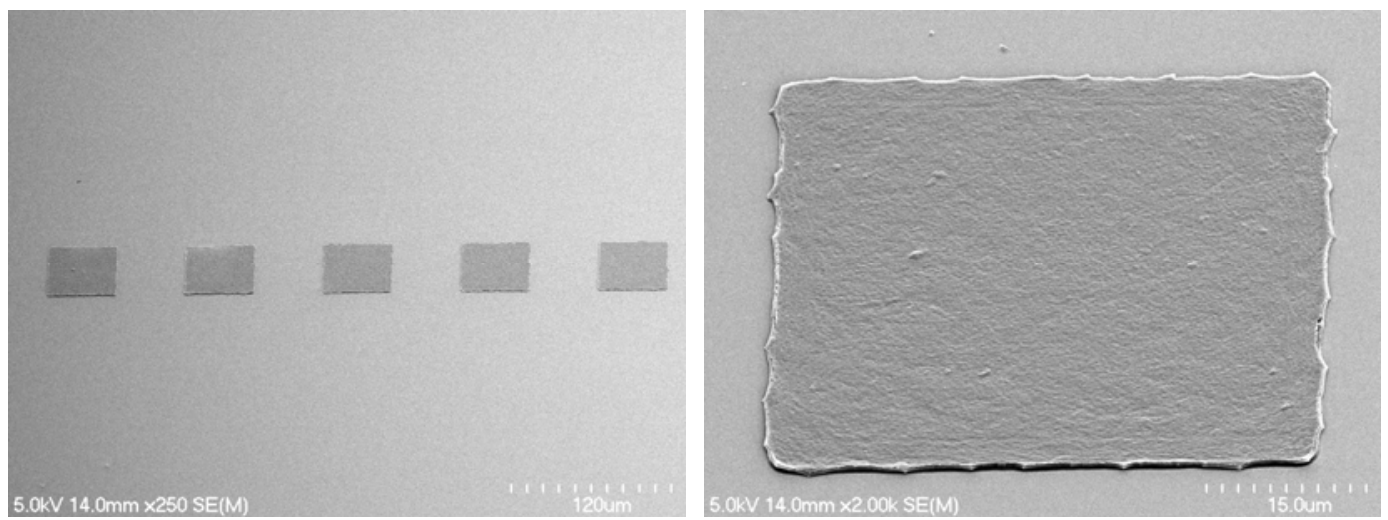

Figure 61: FE-SEM images (left $=120 \mu \mathrm{m}$ scale bar, right $=15 \mu \mathrm{m}$ scale bar) of $50 \mu \mathrm{m}$ square patterned PM films. Accelerating voltage $=5 \mathrm{kV}$, working distance $=12 \mathrm{~mm}$, Stage tilt $=45$ degrees.
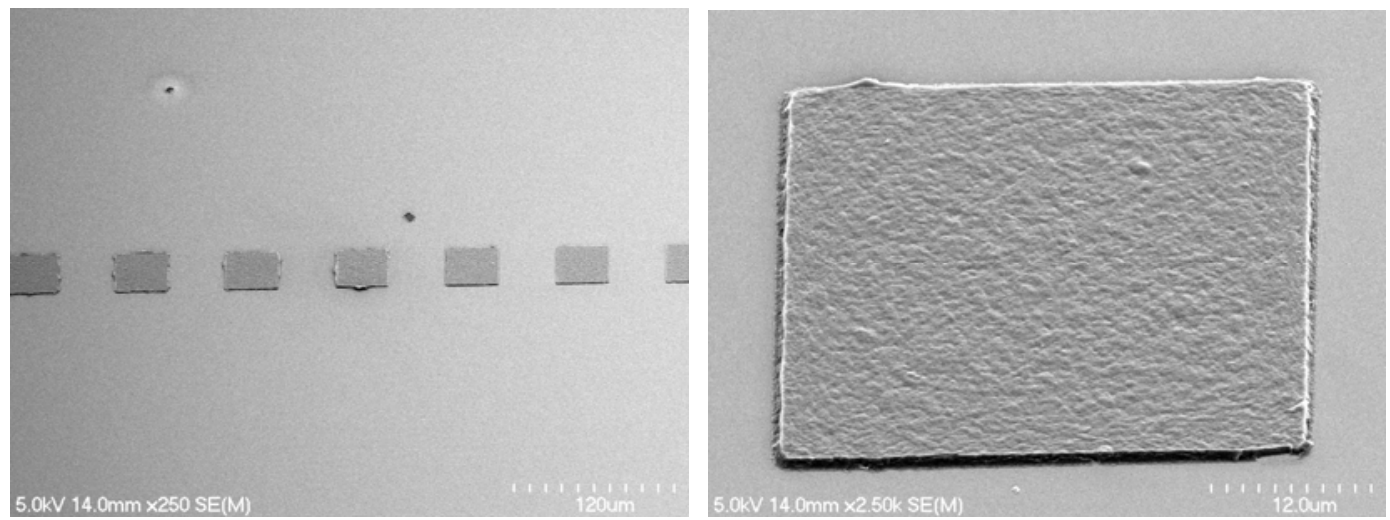

Figure 62: FE-SEM images (left $=120 \mu \mathrm{m}$ scale bar, right $=12 \mu \mathrm{m}$ scale bar) of $40 \mu \mathrm{m}$ square patterned PM films. Accelerating voltage $=5 \mathrm{kV}$, working distance $=12 \mathrm{~mm}$, Stage tilt $=45$ degrees. 

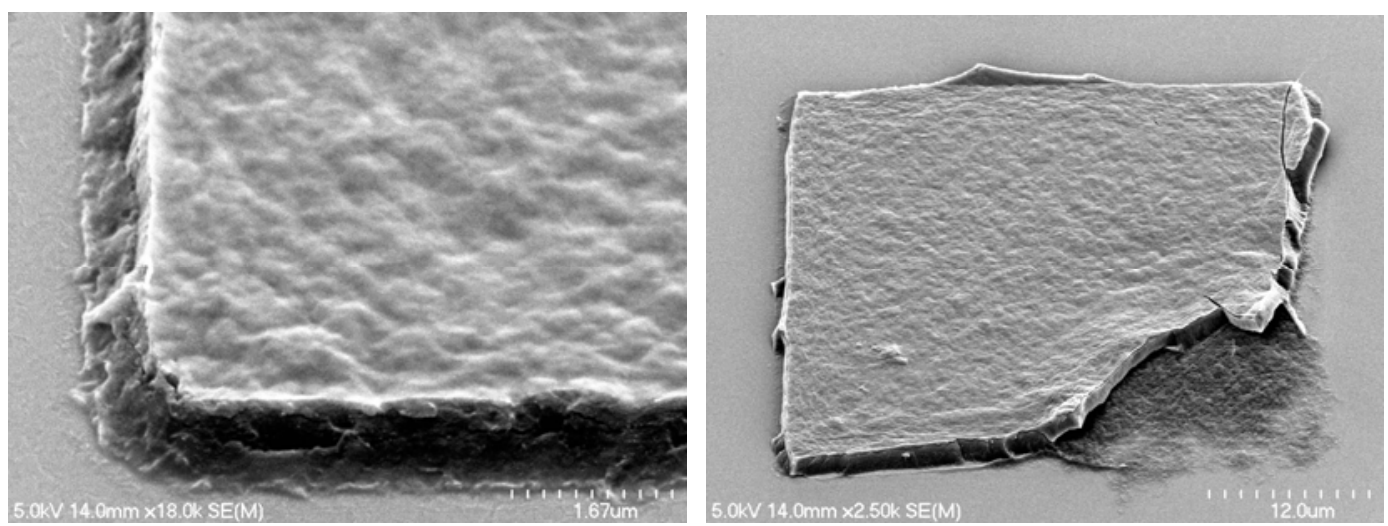

Figure 63: FE-SEM images (left $=1.67 \mu \mathrm{m}$ scale bar, right $=12 \mu \mathrm{m}$ scale bar) of $40 \mu \mathrm{m}$ square patterned PM films. Accelerating voltage $=5 \mathrm{kV}$, working distance $=12 \mathrm{~mm}$, Stage tilt $=45$ degrees.
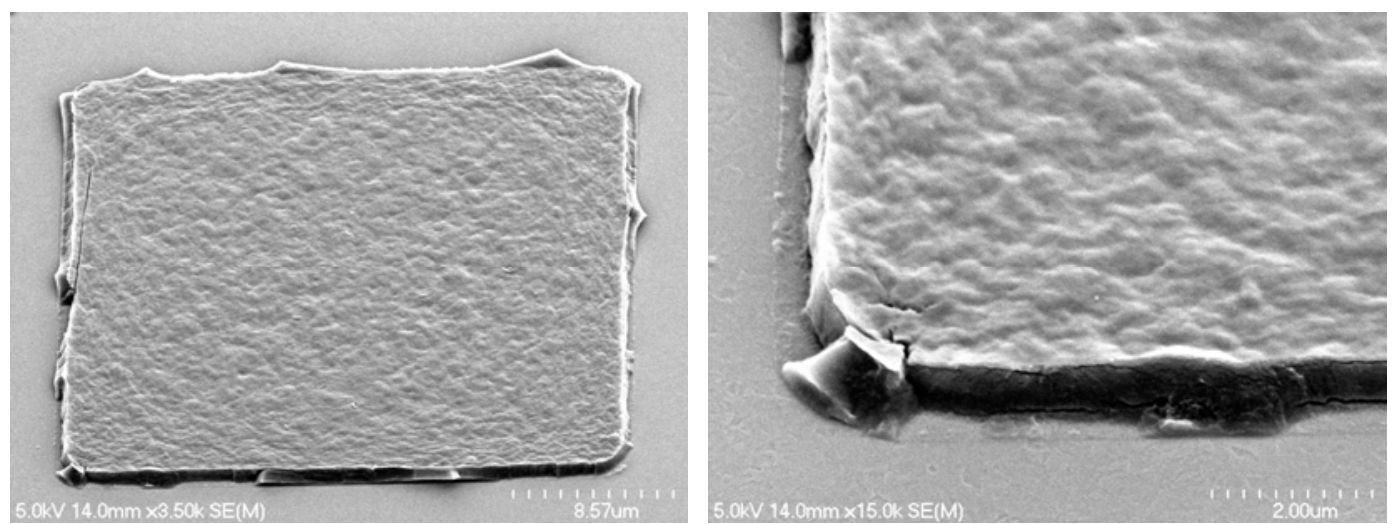

Figure 64: FE-SEM images (left $=8.57 \mu \mathrm{m}$ scale bar, right $=2 \mu \mathrm{m}$ scale bar) of a $30 \mu \mathrm{m}$ square patterned PM film. Accelerating voltage $=5 \mathrm{kV}$, working distance $=12 \mathrm{~mm}$, Stage tilt $=45$ degrees.
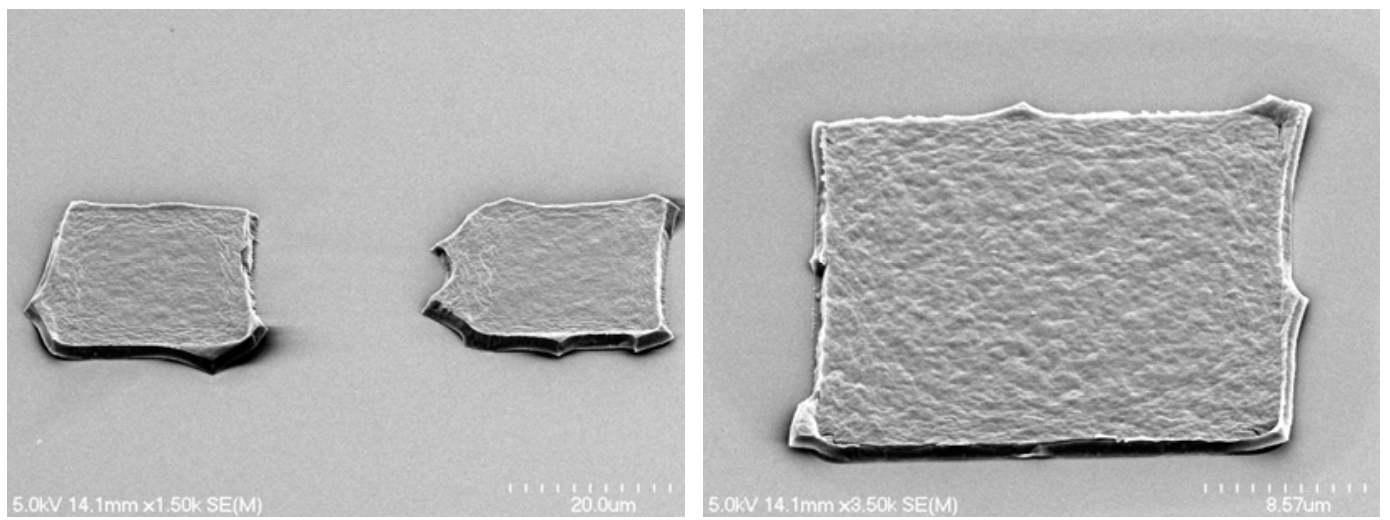

Figure 65: FE-SEM images (left $=20 \mu \mathrm{m}$ scale bar, right $=8.57 \mu \mathrm{m}$ scale bar) of $25 \mu \mathrm{m}$ square patterned PM films. Accelerating voltage $=5 \mathrm{kV}$, working distance $=12 \mathrm{~mm}$, Stage tilt $=45$ degrees. 

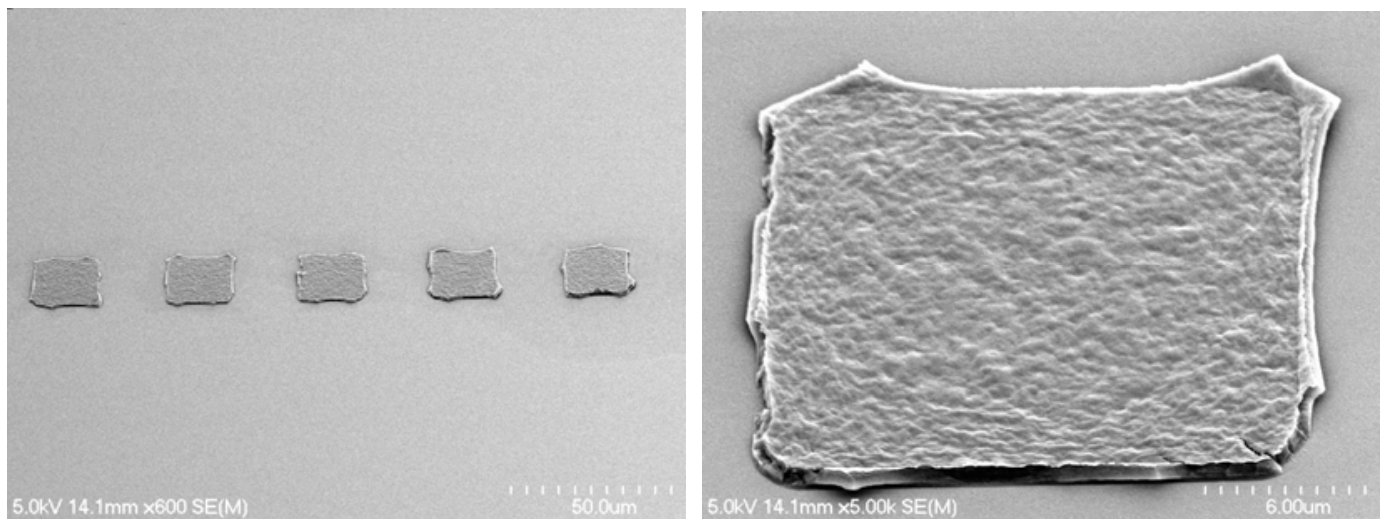

Figure 66: FE-SEM images (left $=50 \mu \mathrm{m}$ scale bar, right $=6.0 \mu \mathrm{m}$ scale bar) of $20 \mu \mathrm{m}$ square patterned PM films. Accelerating voltage $=5 \mathrm{kV}$, working distance $=12 \mathrm{~mm}$, Stage tilt $=45$ degrees.
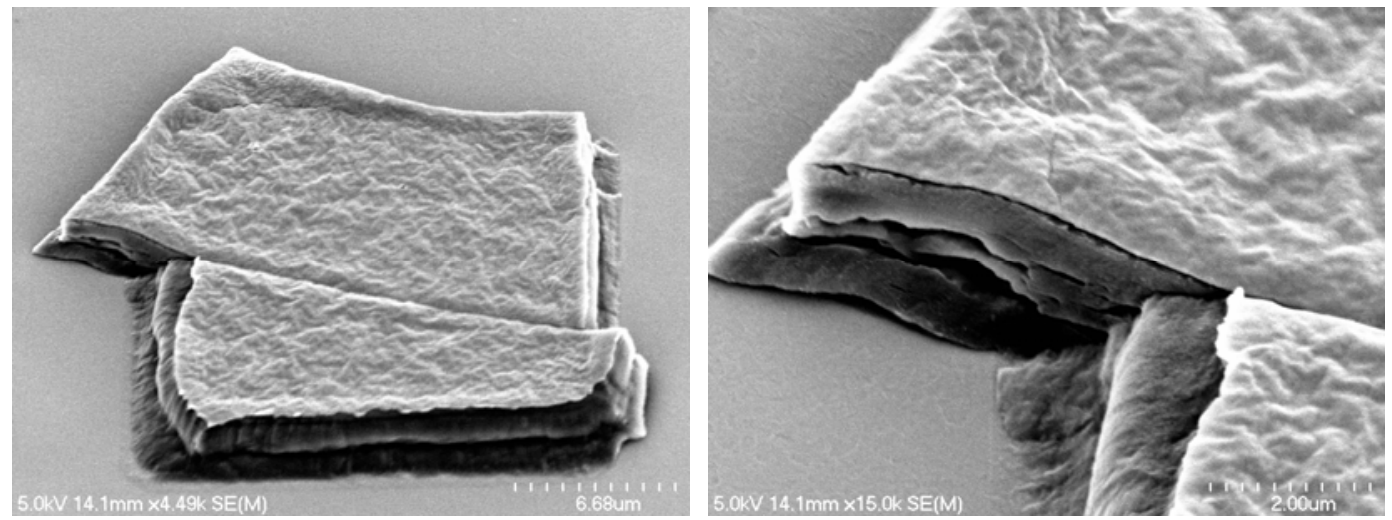

Figure 67: FE-SEM images (left $=6.88 \mu \mathrm{m}$ scale bar, right $=2.0 \mu \mathrm{m}$ scale bar) of a damaged $20 \mu \mathrm{m}$ square patterned PM film. Accelerating voltage $=5 \mathrm{kV}$, working distance $=12 \mathrm{~mm}$, Stage tilt $=45$ degrees.
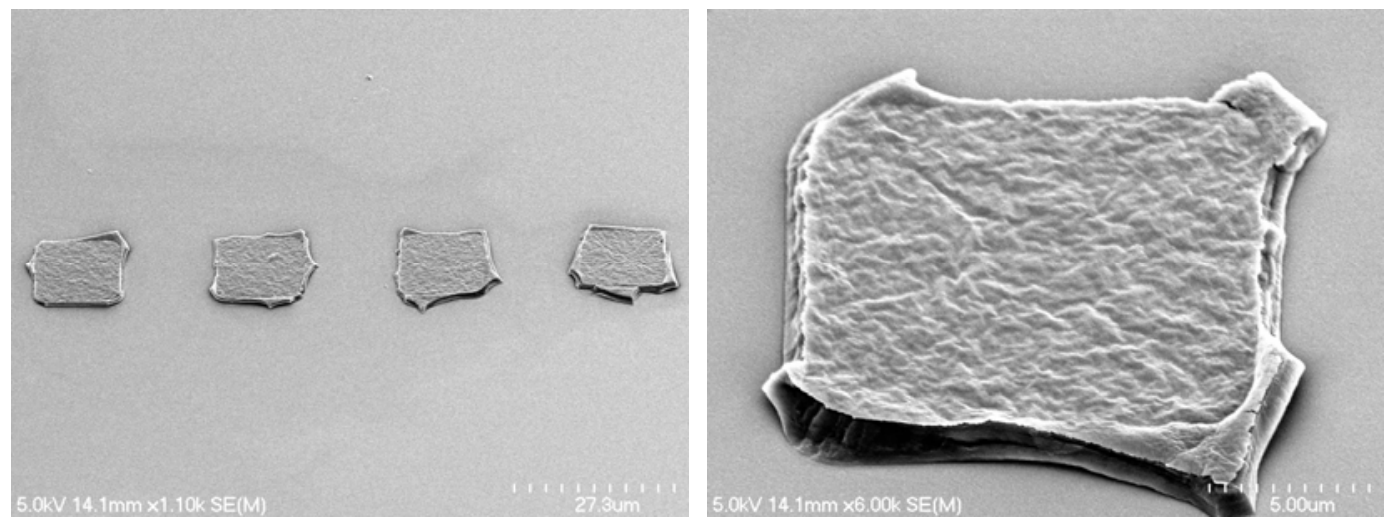

Figure 68: FE-SEM images (left $=27.3 \mu \mathrm{m}$ scale bar, right $=5 \mu \mathrm{m}$ scale bar) of $15 \mu \mathrm{m}$ square patterned PM films. Accelerating voltage $=5 \mathrm{kV}$, working distance $=12 \mathrm{~mm}$, Stage tilt $=45$ degrees . 

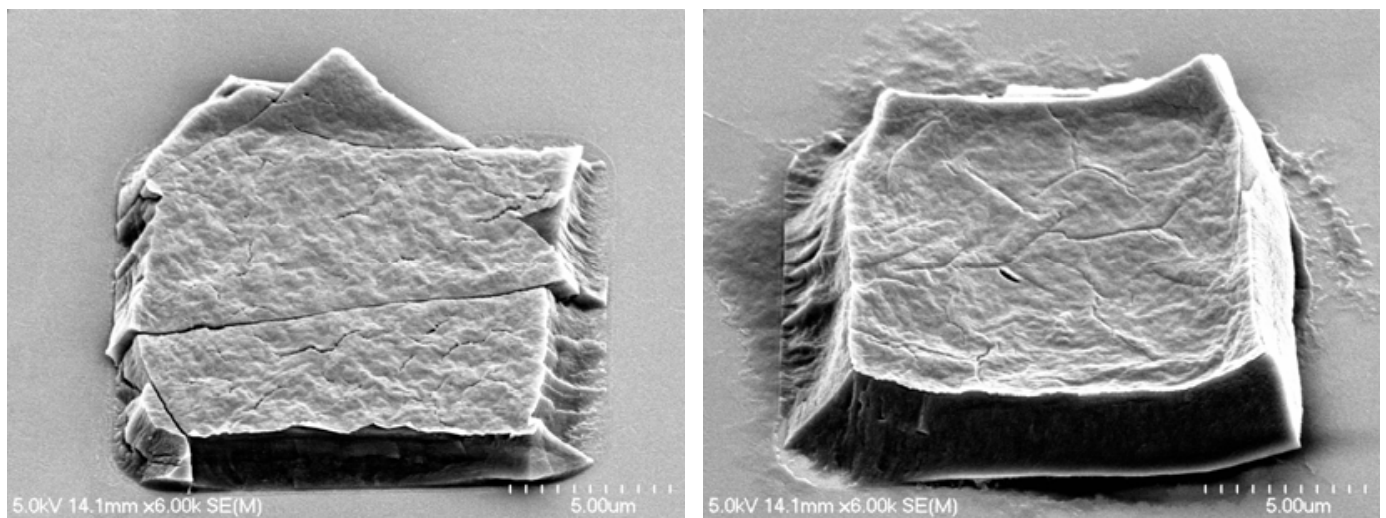

Figure 69: FE-SEM images (left $=5 \mu \mathrm{m}$ scale bar, right $=5 \mu \mathrm{m}$ scale bar) of damaged $15 \mu \mathrm{m}$ square patterned PM films. Accelerating voltage $=5 \mathrm{kV}$, working distance $=12 \mathrm{~mm}$, Stage tilt $=45$ degrees.
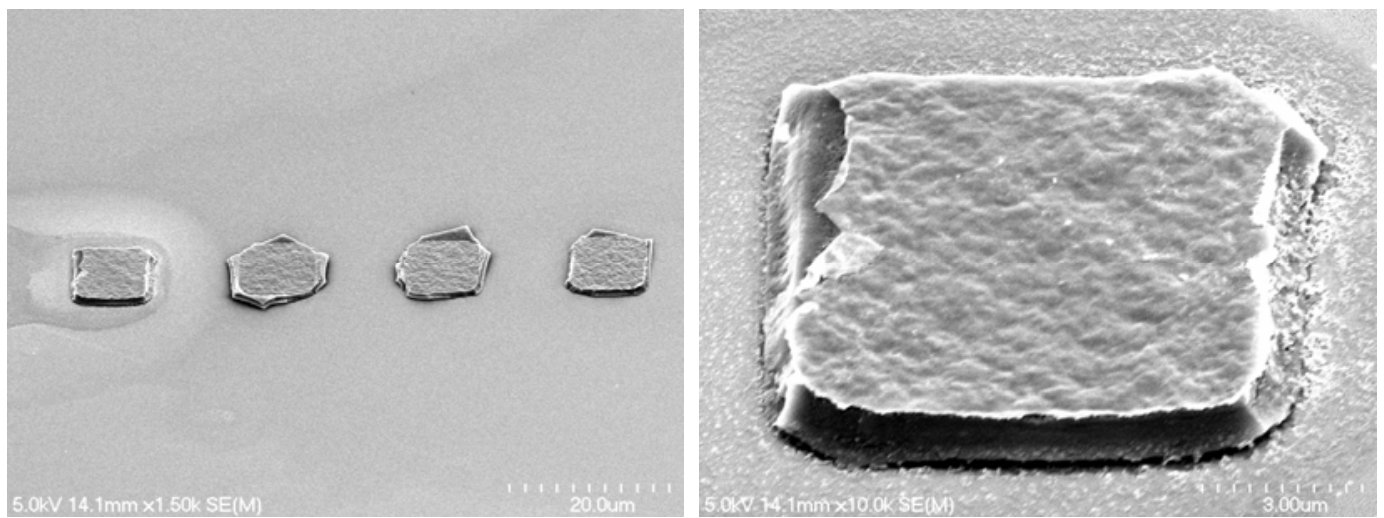

Figure 70: FE-SEM images (left $=20 \mu \mathrm{m}$ scale bar, right $=3.0 \mu \mathrm{m}$ scale bar) of $10 \mu \mathrm{m}$ square patterned PM films. Accelerating voltage $=5 \mathrm{kV}$, working distance $=12 \mathrm{~mm}$, Stage tilt $=45$ degrees.
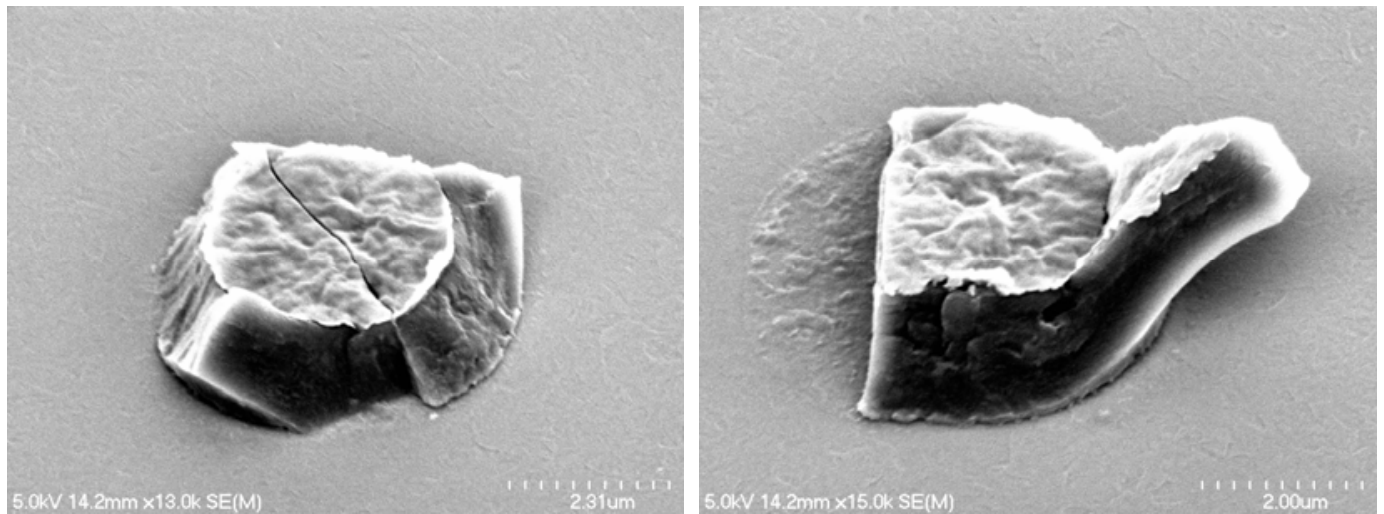

Figure 71: FE-SEM images (left $=2.31 \mu \mathrm{m}$ scale bar, right $=2.0 \mu \mathrm{m}$ scale bar) of $5 \mu \mathrm{m}$ square patterned PM films. Accelerating voltage $=5 \mathrm{kV}$, working distance $=12 \mathrm{~mm}$, Stage tilt $=45$ degrees. 
Figure 60 through Figure 71 show the patterned PM films produced on a single substrate by the PBBPT. Based on the FE-SEM images of the films, the practical feature size limit of the PBBPT is $15 \mu \mathrm{m}$. A large number of defects were present around the outer edges of the $10 \mu \mathrm{m}$ squares. Many of the squares also showed damage. Most of the $5 \mu \mathrm{m}$ squares were missing, indicating either a limitation of the electrodeposition process or a limitation of the patterning process. The size of individual PM patches in used in the deposition process (100 $\mathrm{nm}-5 \mu \mathrm{m}$ ) most likely limit the minimum feature size of patterned PM films. The $5 \mu \mathrm{m}$ features that were present were irregular in shape, with no straight edges indicating a square pattern.

Although it is unclear what the exact dimensions of the PM sensing sites will be on the final sensing platform, they will most likely be larger than $15 \mu \mathrm{m}$. Increasing the surface area of the sensing sites will lead to better probability of detecting an analyte of interest. Because of this, it is unlikely that the PBBPT will be the limiting factor in the overall size of the detection platform.

\subsection{Variations of the PBBPT}

In its initial form, the PBBPT required a conductive substrate that is accessible to facilitate the electrodeposition process. This process flow was used because it involved the least number of processing steps, and thus the fewest number of process variables to control. Once the basic process was understood and optimized, several modifications to the process were made to make the process more robust. The first modification allowed for PM films to be deposited onto non-conductive substrates. The second modification allowed for a conductive coating to be deposited on top of the patterned PM films, allowing for a top electrode over the PM film. 


\subsubsection{PBBPT onto Non-Conductive Substrates}

The purpose of developing the PBBPT was to use the technique to integrate PM films with microelectronic devices. These devices will most likely be fabricated onto semiconducting substrates such as silicon. Because of this, it is advantageous to be able to pattern and deposit PM films onto non-conductive substrates. To accomplish this, an extra processing step was added to the PBBPT process. The new process flow is shown in Figure 72.

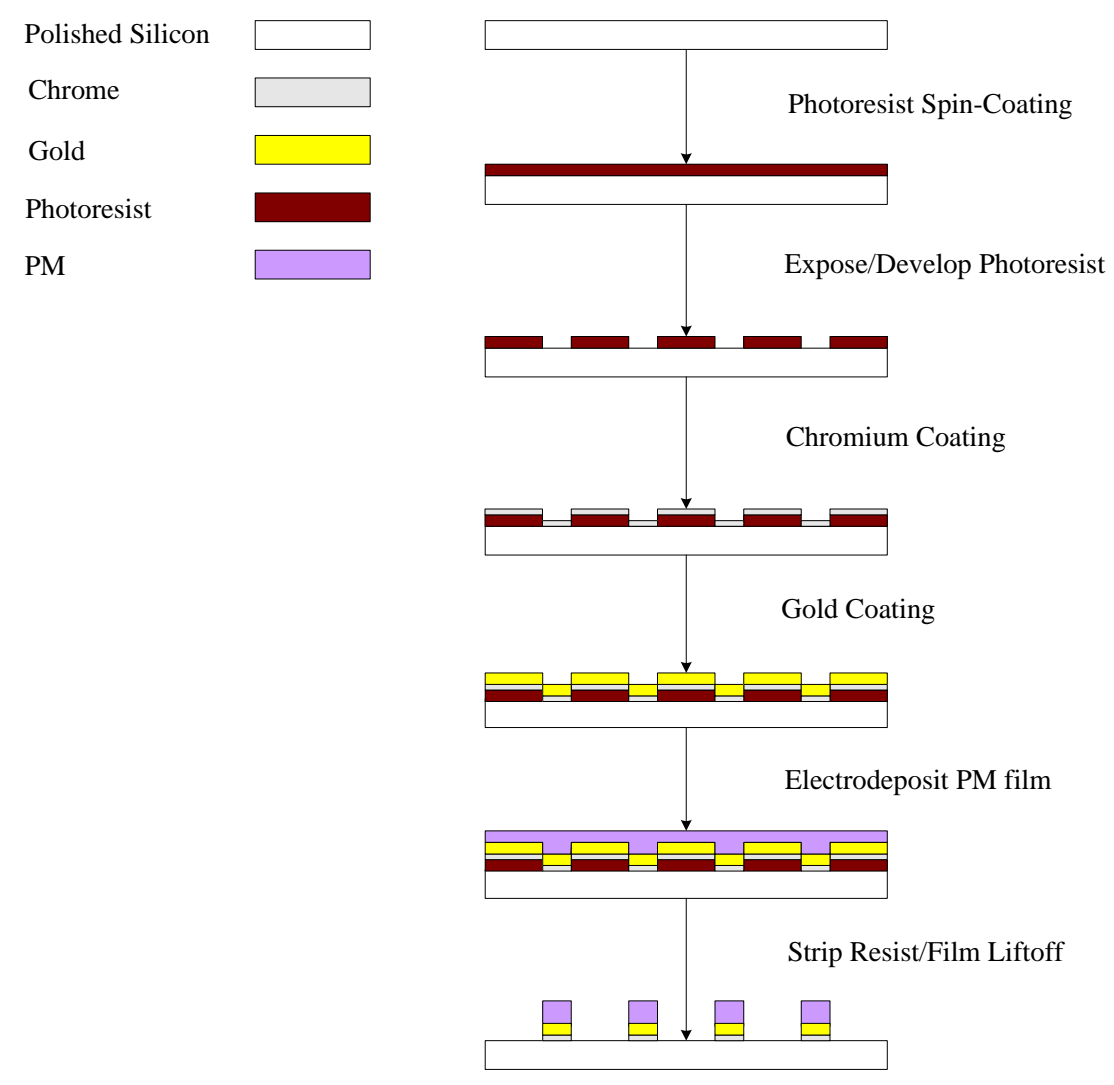

Figure 72: Schematic representation of a revised PBBPT process flow that allows for PM deposition and patterning onto non-conductive substrates.

The change in the process flow is the addition of a metallic film coating after resist development, and prior to PM electrodeposition. The thin film covers the entire 
substrate, both the areas covered with photoresist and the exposed areas. Following the metal film deposition, a PM film is electrodeposited over the entire patterned region of the substrate. Because the entire substrate is conductive, the PM film deposits over the entire PM suspension area. The photoresist is removed from the substrate by an acetone sonication bath, leaving patterned PM films over a patterned conductive metal film.

To confirm this revised process flow, the process was carried out and the resulting films were analyzed using an IFM. A patterned substrate was used from the set discussed in Section 4.1.2. The substrate was coated with $20 \mathrm{~nm}$ of Au using the Perkin-Elmer Model 6J RF sputtering system. Electrodeposition was then performed over the patterned region of the substrate. A $15 \mu \mathrm{l}$ drop of suspended PM $(13.9 \mathrm{mg} / \mathrm{ml})$ was placed on the substrate. Gold coated silicon was used as the top electrode, and was separated from the substrate by a $1.2 \mathrm{~mm}$ spacer. A voltage of 4.0 volts was applied for 45 seconds. The substrate was rinsed in ddi water and allowed to dry at 56\% humidity for 24 hours. After drying, the sample was submerged in an acetone sonication bath for 8 minutes. The extended sonication time was to allow for the complete removal of the unwanted PM film and gold layer. After the liftoff process was complete, the sample was sputter coated with an additional $20 \mathrm{~nm}$ of Au to facilitate IFM analysis. 


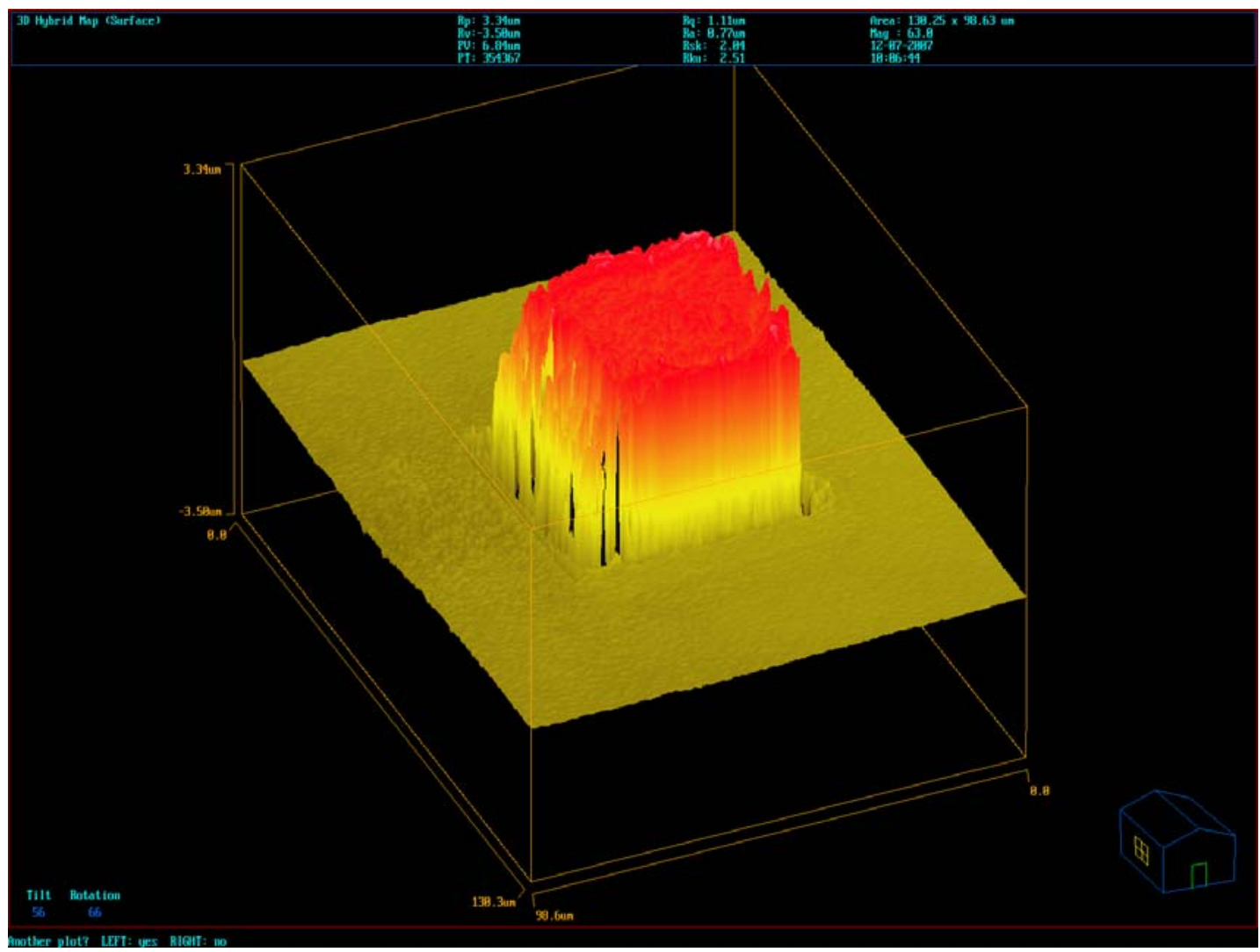

Figure 73: IFM scan (63X mag) of a patterned PM film produced using the revised PBBPT process that allows for deposition onto non-conductive substrates. Maximum thickness $\sim 3.3 \mu \mathrm{m}$.

Figure 73 shows a single patterned PM film from the revised PBBPT process flow. The PM film shows good pattern replication, with straight sides. The film was approximately $3.3 \mu \mathrm{m}$ thick, indicating that the PM film was retained on the surface of the substrate while the undesired PM film was removed by the liftoff process. This result demonstrates the effectiveness of the revised PBBPT process, making the process much more versatile when considering the integration of PM films with existing microelectronic devices. 


\subsubsection{PBBPT with a Conductive Coating on Top of the PM Films}

A second alteration to the basic PBBPT process involved the addition of a thin gold film coated on top of the electrodeposited PM film prior to the liftoff step. Gold was chosen because of its high electrical conductivity, and its convenient availability on site. The purpose of this gold film was to provide electrical access to the top of the PM film for the purpose of grounding the PM film to the shared ground of whatever microelectronic device the PM is to be integrated with. A film thickness of $\sim 8 \mathrm{~nm}$ of gold was chosen, which provided a highly conductive film that still allowed light to pass through the film to the PM. It is critical that whatever material is coated on top of the PM allows light to pass through the film and activate the PM. 


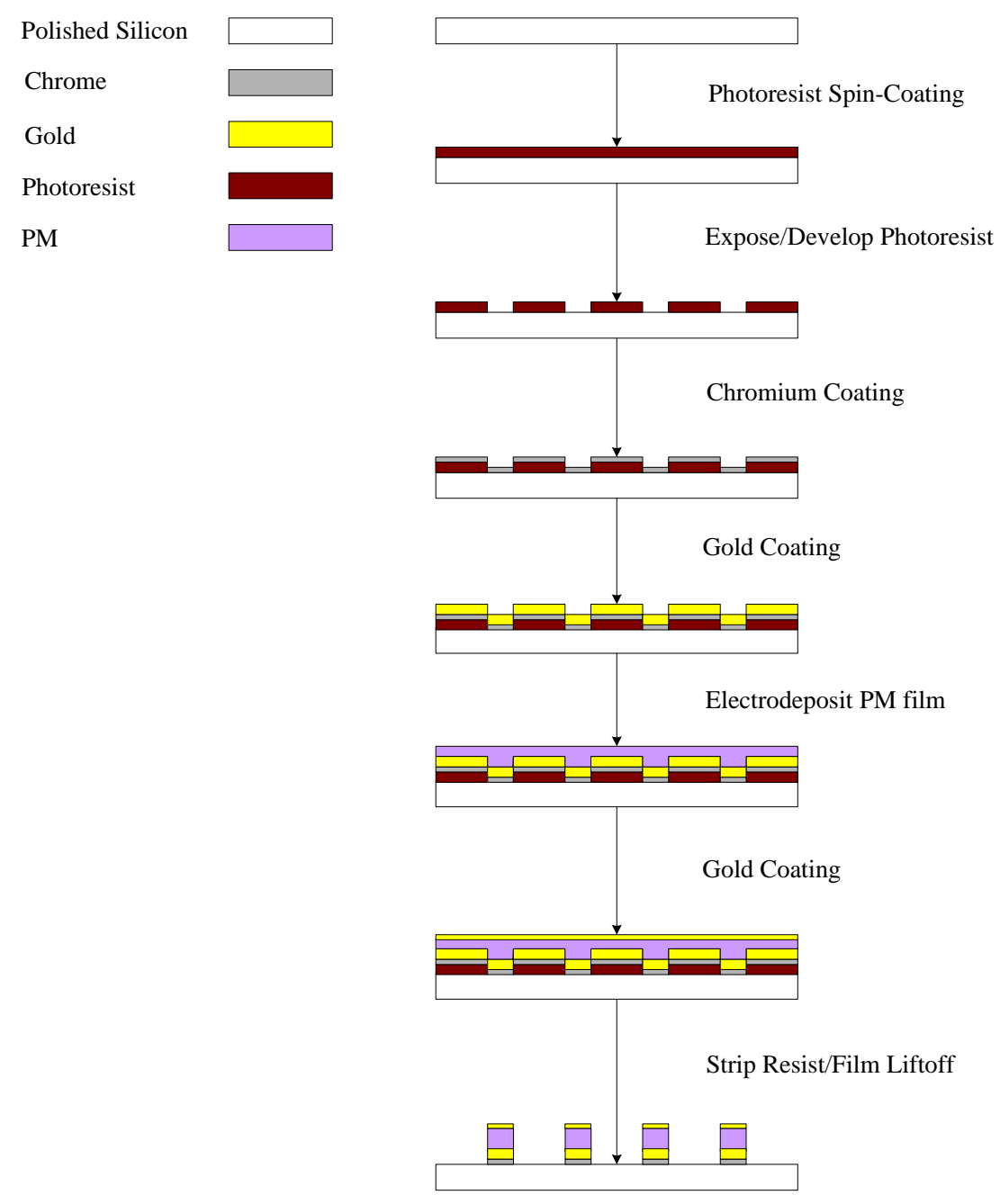

Figure 74: Schematic representation of the revised PBBPT process flow allowing for PM films coated with a thin metallic film to be patterned onto non-conductive substrates.

Figure 74 shows a schematic representation of the revised PBBPT process. FE-SEM images of patterned PM films produced by this revised process were shown in Section 4.2.3. These results showed that the addition of a thin gold film on top of the PM films prior to liftoff still allows for excellent pattern generation. The photoresponse of PM films patterned using the process flow described above is discussed in Sections 5.3 thru 5.5. The results shown in these sections confirm that the functionality of the PM films patterned using this revised PBBPT process flow is maintained. 


\subsection{Confirmation of Patterned PM Film Electrical Activity}

For a PM patterning technique to be successful, it must result in patterned PM films that continue to translate protons when exposed to light. As a final test of the PBBPT process, an experiment was performed to confirm the photoelectric activity of PM films patterned using the technique. To perform this experiment, a new photolithography mask was required with larger feature sizes than the mask used in previous studies. A mask was designed using Cadence, which consisted of squares $0.5 \mathrm{~mm}$ on a side, and increased by $0.5 \mathrm{~mm}$ increments up to $4.0 \mathrm{~mm}$ on a side. Because of the large feature sizes, a transparency mask was fabricated in house using a drum printer. This mask making process has a feature size limitation of $\sim 30 \mu \mathrm{m}$. The advantages of this process were that no costs were incurred to make the mask, and the mask making process took $~ 3$ hours.

ITO coated glass slides were used as the substrates in this experiment. Each slide was cleaned using acetone followed by IPA. A 2-minute pre-bake at $140^{\circ} \mathrm{C}$ was performed to remove any solvents from the substrate surface. After cleaning, the samples were coated with Futurrex 2000A photoresist. This was done at 6000 RPM for 3 seconds using a spin coater. Because the spin coater took time to accelerate to 6000 RPM, each sample was spun for 3 seconds after the spinner reached the target speed. This high photoresist spin speed was used because of the small size (5 mm X $20 \mathrm{~mm}$ ) of the ITO slides. When a slower spin speed was used, photoresist pooled at the four corners of the substrate during the spin, resulting in a non-uniform photoresist layer.

After the resist was coated onto the samples, a 5 minute soft bake at $140^{\circ} \mathrm{C}$ was performed in order to cure the photoresist. The transparency mask was taped to a 125 mm glass plate. A tape covered metal frame used to secure small samples during the alignment process was laid on top of the mask. Eight samples were placed on the tape directly over the eight square patterns on the mask. The mask and the tape-covered frame were loaded into the aligner, and the mask and substrates were aligned visually. The 
aligner was used in proximity mode with a $50 \mu \mathrm{m}$ proximity to avoid the transparency and the substrates sticking to one another. The samples were exposed to the UV aligner light for 12 seconds. Following UV exposure, the samples were developed in Futurrex RD6 resist developer for 10 seconds, followed by rinsing in ddi water.

After the ITO slides were patterned, PM films were deposited onto the substrate by electrodeposition. The photoresist was removed from a corner of each sample using acetone to facilitate the electrodeposition process. A liquid suspension of PM (2.52 $\mathrm{mg} / \mathrm{ml}$ ) was placed on top of the patterned region of each sample individually. A $15 \mu \mathrm{l}$ drop was used on the $0.5 \mathrm{~mm}, 1 \mathrm{~mm}, 1.5 \mathrm{~mm}, 2 \mathrm{~mm}$, and $2.5 \mathrm{~mm}$ samples. A $20 \mu \mathrm{l}$ drop was used on the $3 \mathrm{~mm}$ and $3.5 \mathrm{~mm}$ samples. A $25 \mu \mathrm{l}$ drop was used on the $4 \mathrm{~mm}$ sample. The purpose of the varying PM drop sizes was to cover the entire pattern without using excess PM. Gold coated silicon was used as the top electrode, and a $1.2 \mathrm{~mm}$ spacer was placed between the substrate and the top electrode. 4.0 volts was applied between the substrate and the top plate for 40 seconds to achieve deposition. No PM deposition was observed on the $0.5 \mathrm{~mm}$ and $1.0 \mathrm{~mm}$ samples. This may have been caused by an incomplete development during lithography, which would have left a thin film of photoresist on the patterned area of the substrates. The $2 \mathrm{~mm}$ sample was rinsed in ddi water immediately following electrodeposition, which resulted in the removal of some of the PM film from the patterned region. The remaining 5 samples were not rinsed after PM deposition.

To determine the impact of the PBBPT on the electrical output of the PM films, electrical output measurements due to light were taken under the same experimental conditions both before and after the acetone sonication bath. Each sample was measured for electrical activity individually using a custom test apparatus developed by another research group member. The samples were loaded into a mounting chuck, which consisted of a machined aluminum frame, a machined plastic clamp and a microstage. The mounting chuck was used to bring the PM film into contact with a second ITO glass 
plate, which acted as the top electrode during the electrical measurements. The resistance between the two plates was tested before each measurement was taken to ensure that the two plates were not touching.

The mounting chuck was placed in an aluminum box that was grounded to reduce electrical noise. The top and bottom plates were attached to a male co-axial cable coupler that was accessible from outside of the box. The electrical signals produced by the PM were sent through a co-axial connection to a custom built operational amplifier with a high impedence (1 Tara Ohm), and a gain of 11X. A high impedance amplifier was necessary to measure the PM response due to the high resistivity of the PM film, as well as the small current produced by the film. The signal was fed from the amplifier to an Agilent 54622A Oscilloscope, where it was measured and recorded in DC mode.

An LED light source consisting of 3 amber LEDs (595 nm peak emission) was used as the light source for this experiment. The LEDs provided illumination in the peak absorbance wavelength of the PM films. The LEDs were powered by a power supply at 30 volts through a custom built circuit. The current supplied to the LEDs could be modulated in order to alter the amount of light emmited by the LEDs. A current of 0.4 amps was used to power the LEDs during all of the measurements taken in this experiment. The LED array was located at a distance of $62 \mathrm{~mm}$. An optical power measurement under this lighting condition was taken using a Thor Labs PM100 optical power meter, and was found to be $6.804 \mathrm{~mW} / \mathrm{cm}^{2}$ at a wavelength of $570 \mathrm{~nm}$. A secondary circuit was combined with the power circuit to provide pulsing of the light source. The LEDs were pulsed at a frequency of $0.5 \mathrm{~Hz}$ with a duty cycle of $50 \%$ for all electrical measurements.

Each sample was measured for electrical activity under the experimental conditions described above. The oscilloscope was used to capture 20 seconds of data for each sample. The samples were then placed in an acetone sonication bath (103 W, $43 \mathrm{kHz}$ ) for 
various amounts of time. Each sample was removed from the sonication bath when the square patterned PM was clearly defined. The samples were placed back into the experimental setup and an additional 20 second oscilloscope reading was taken. Because deposition onto the $0.5 \mathrm{~mm}$ and $1.0 \mathrm{~mm}$ samples were unsuccessful, only six samples were used in this experiment. Raw data from the oscilloscope was imported into Excel, and plots of PM voltage vs. time were made for each sample both before and after the acetone sonication bath. An example of the voltage response of the PM film before and after sonicaiton is shown Figure 75 and Figure 76 respectively.

\section{$4 \mathrm{~mm} \times 4 \mathrm{~mm}$ PM Square Pre-Strip}

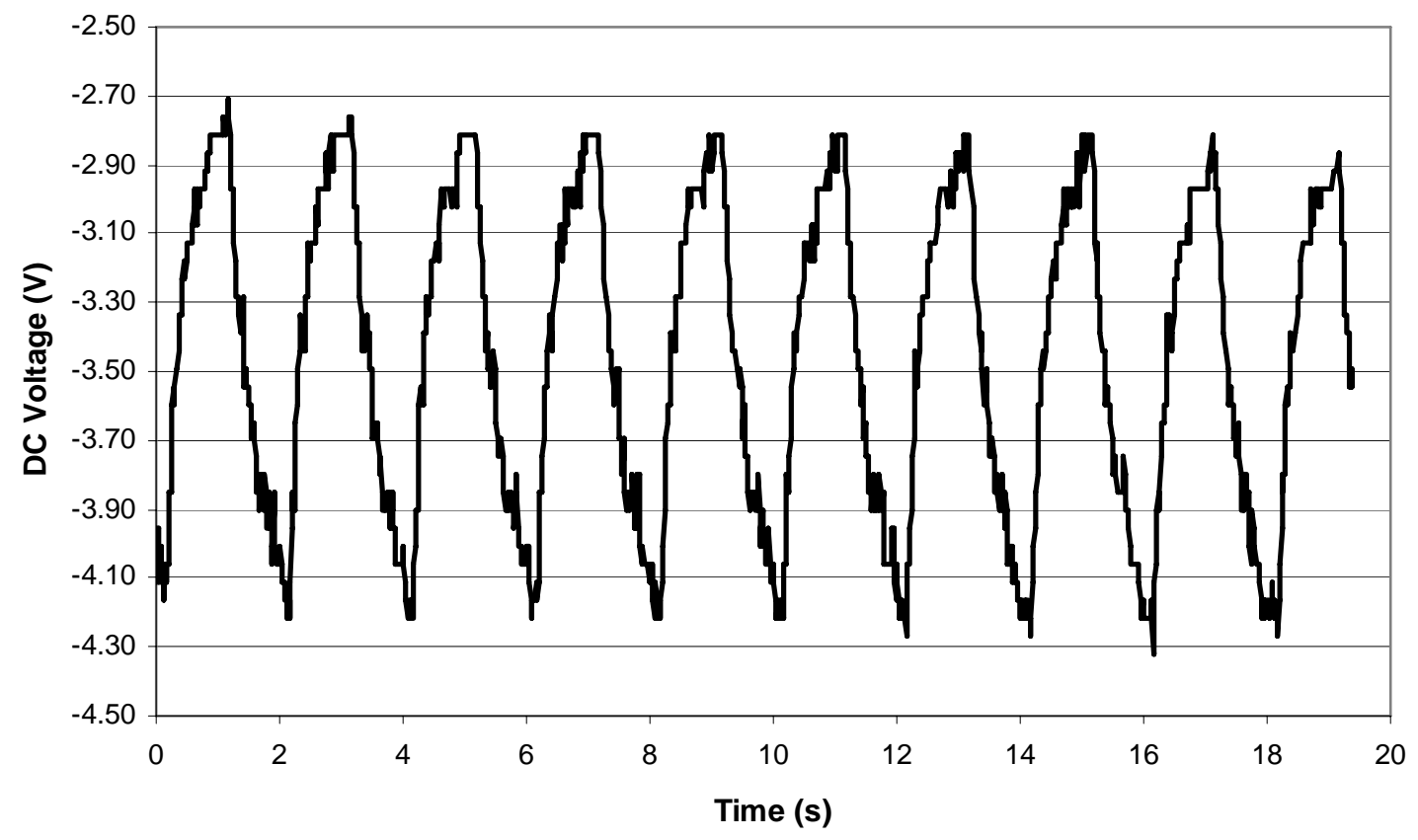

Figure 75: DC voltage response of pre-acetone sonication bath $4 \mathrm{~mm}$ PM square film to pulsed LED light. Upward curves indicate the light source was on, downward curves indicate the light was off. 3-point moving average trend line used. 


\section{4mm x 4mm PM Post Strip}

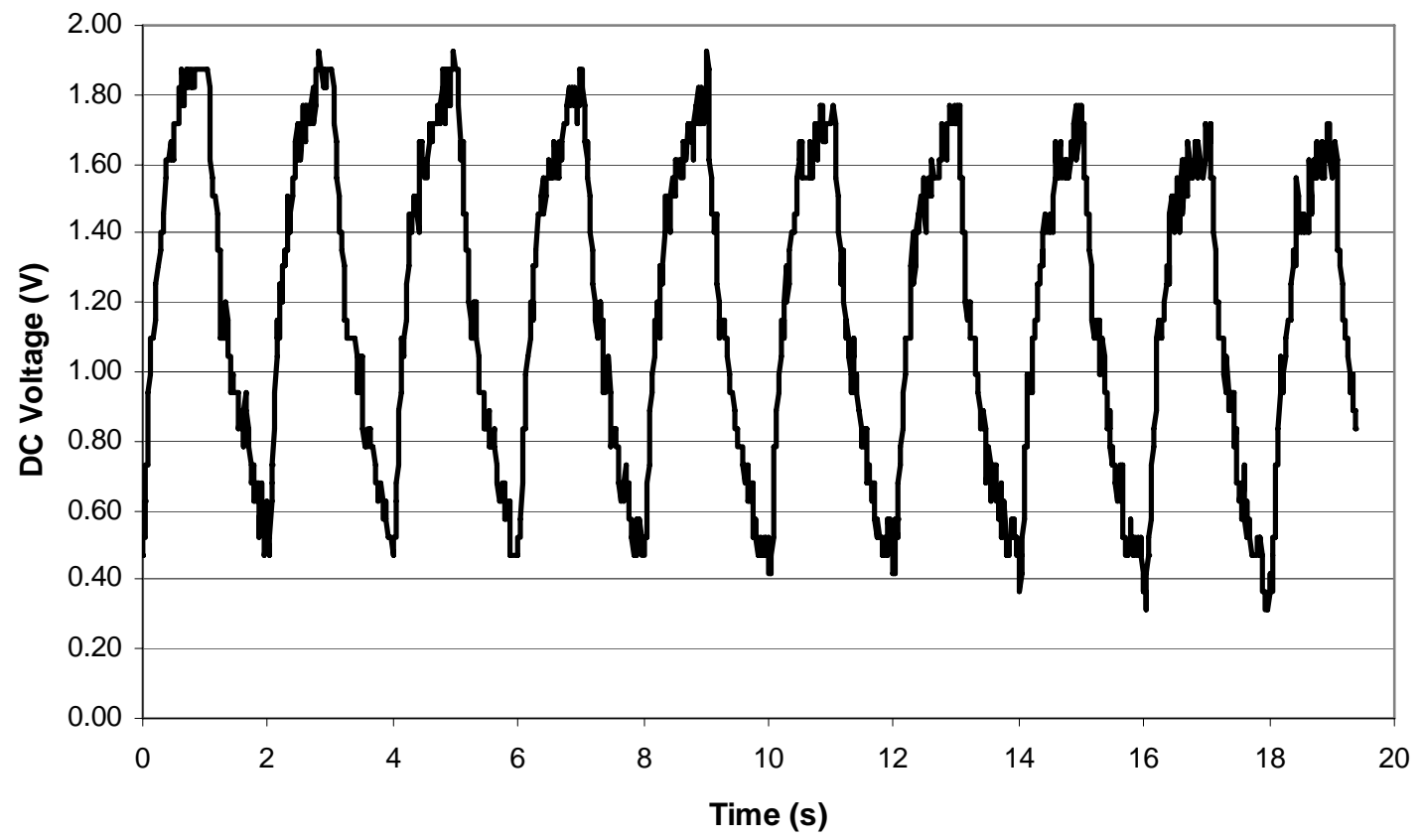

Figure 76: DC voltage response of post-acetone sonication bath $4 \mathrm{~mm}$ PM square film to pulsed LED light. Upward curves indicate the light source was on, downward curves indicate the light was off. 3-point moving average trend line used.

The characteristic of interest in the two graphs is the magnitude of the peak to valley voltage. A three-point moving average trend line was applied to the above graphs to smooth the graphs. A positive photovoltage was produced upon illumination from the LEDs, resulting in the upward trends seen in the measured photovoltages in Figure 75 and Figure 76. The low points along the voltage trace relate to the light turning on, while the high points on the graph relate to the light turning off. This test setup introduced some drift in the signal. This drift can be seen in Figure 76 as a downward trend in both the peaks and valleys of the PM voltage response. This voltage drift also resulted in large variations in the sign and magnitude of the baseline measurements obtained with no light supplied to the PM film, resulting in a large sign and magnitude difference between the photovoltages shown in Figure 75 and Figure 76. 
It was unclear if the drift was due to the PM films or the operational amplifier. This signal drift also causes variation in the overall voltage recorded by the oscilloscope. Neither of these trends in the captured data had a negative impact on the experiment, as the difference between the peak and valley voltage values are all that were considered. The average peak to valley voltage for all of the pre-patterned and post-patterned samples along with the acetone sonication time for each sample are compiled in Table 4.

Table 4: Acetone sonication time, pre-photoresist strip voltage, post-photoresist voltage and change in voltage after the photoresist strip are shown. Voltages shown represent average peak to valley voltages obtained from oscilloscope data.

\begin{tabular}{|c|c|c|c|c|}
\hline $\begin{array}{c}\text { Substrate } \\
\text { Square } \\
\text { Pattern Size }\end{array}$ & $\begin{array}{c}\text { Acetone } \\
\text { Sonication } \\
\text { Time }\end{array}$ & $\begin{array}{c}\text { Pre-Strip } \\
\text { Voltage }\end{array}$ & $\begin{array}{c}\text { Post-Strip } \\
\text { Voltage }\end{array}$ & $\begin{array}{c}\text { Change in Voltage } \\
\text { After Strip }\end{array}$ \\
\hline $4.0 \mathrm{~mm}$ & $3 \mathrm{~min}$ & $1.410 \mathrm{~V}$ & $1.410 \mathrm{~V}$ & 0 \\
\hline $3.5 \mathrm{~mm}$ & $8 \mathrm{~min}$ & $0.940 \mathrm{~V}$ & $1.875 \mathrm{~V}$ & 0.935 \\
\hline $3.0 \mathrm{~mm}$ & $12 \mathrm{~min}$ & $0.938 \mathrm{~V}$ & $2.500 \mathrm{~V}$ & 1.562 \\
\hline $2.5 \mathrm{~mm}$ & $12 \mathrm{~min}$ & $0.781 \mathrm{~V}$ & $1.719 \mathrm{~V}$ & 0.938 \\
\hline $2.0 \mathrm{~mm}$ & $9 \mathrm{~min}$ & $1.250 \mathrm{~V}$ & $1.094 \mathrm{~V}$ & -0.156 \\
\hline $1.5 \mathrm{~mm}$ & $9 \mathrm{~min}$ & $0.625 \mathrm{~V}$ & $0.625 \mathrm{~V}$ & 0 \\
\hline
\end{tabular}

As can be seen in Table 4, the acetone sonication bath did not negatively impact the photovoltage produced by the PM films. This result is significant, as it demonstrates that the PBBPT is capable of patterning PM films without negatively impacting the photoelectric properties of the film. The $2.5 \mathrm{~mm}, 3 \mathrm{~mm}$, and $3.5 \mathrm{~mm}$ samples showed large increases in voltage due to light pulsing after the acetone sonication bath. This variation may be due to the acetone sonication bath removing un-oriented PM patches that may have been present on top of the samples. Because these three samples were not rinsed immediately after sonication, unbound non-oriented PM patches most likely were dried on top of the oriented film. It is possible that the sonication in acetone removed some of these un-oriented films, which also increased the amount of light reaching the oriented film below. It is also possible that these variations were due to variations in the 
contact between the ITO top plate and the PM samples before and after the acetone sonication bath. However, these hypotheses were not investigated. Despite these variations in PM photovoltage output, this study does demonstrate that the photovoltage produced by PM films is not reduced or eliminated by the PBBPT.

\subsection{Impact of Elevated Temperatures on PM Photovoltage Output}

One variable that must be considered when working with biological materials is temperature. Membranes such as PM tend to loose their functionality when exposed to elevated temperatures. Understanding the impact of elevated temperatures on PM films will help to determine which fabrication processes the PM films can survive.

Three ITO coated glass slides were patterned by photolithography using the protocol described in Section 4.4. The patterned slides each had a single square in the center of the slide, and the sizes of the squares were $2.0 \mathrm{~mm}, 2.5 \mathrm{~mm}$ and $3.0 \mathrm{~mm}$. Electrodeposition of PM films onto the three squares was performed in the same manner described in Section 4.4. After the PM films had dried, a $\sim 7 \mathrm{~nm}$ thick film of gold was deposited onto each of the substrates. This was followed by 6 minutes in an acetone sonication bath to remove the resist and pattern the membranes and gold films.

The SPA was used to obtain photovoltage measurements. A two probe program with a collector and an emitter from the SPA software was used to perform the measurements. A tri-axial probe was used to contact the ITO substrate, and was set as the collector with a $0 \mathrm{~V}$ bias. A second tri-axial probe contacted the thin gold film on top of the PM, and was set as the emitter. The emitter was set to ground. The LED array was positioned $\sim 125 \mathrm{~mm}$ from the sample at a 45 degree angle from the substrate normal. A current of 1.02 A was used to drive the LED array. This lighting setup resulted in the sample being exposed to light with an optical power of $2.335 \mathrm{~mW} / \mathrm{cm}^{2}$ at a wavelength of $570 \mathrm{~nm}$. The light source was modulated manually by turning the power supply on and off. 
Initial measurements were taken for each of the three samples at room temperature $\left(23^{\circ} \mathrm{C}\right)$. A hot plate was used to increase the temperature of the three samples. A thermocouple was used to determine the temperature of the hot plate surface. After the room temperature measurements were taken for each sample, the three samples were placed on the hot plate for 120 seconds at $80^{\circ} \mathrm{C}$. The samples were taken off the hot plate and allowed to cool back to room temperature. The samples were then connected to the SPA, pulsed with $0.5 \mathrm{~Hz}$ LED pulses, and photovoltage measurements were taken of each sample. The process was repeated with the hot plate temperature at $90^{\circ} \mathrm{C}, 100^{\circ} \mathrm{C}$, $110^{\circ} \mathrm{C}, 120^{\circ} \mathrm{C}$, and $130^{\circ} \mathrm{C}$. Figure 77 shows an example of the PM photovoltage captured using the SPA.

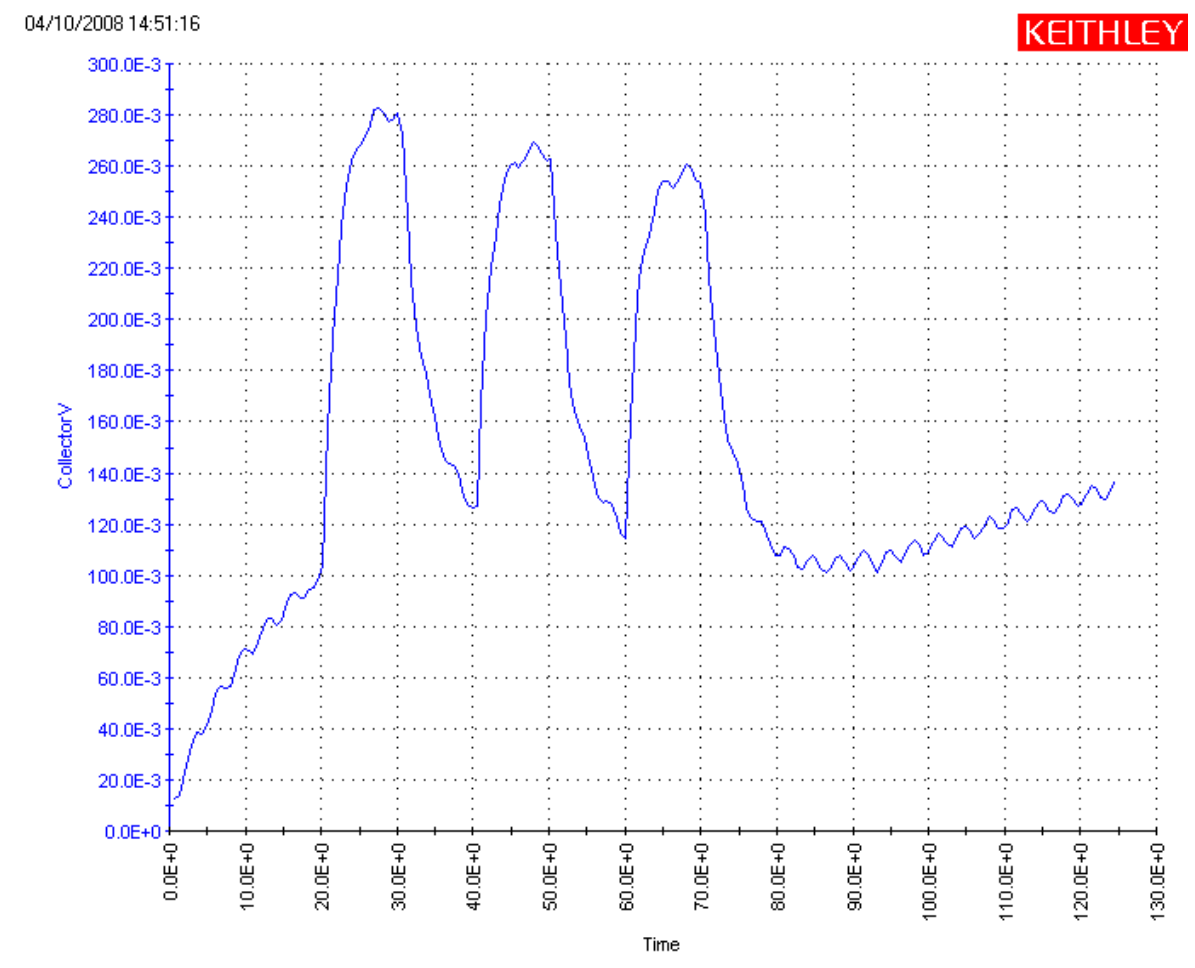

Figure 77: $2.5 \mathrm{~mm}$ patterned PM square photovoltage after exposure to a $90^{\circ} \mathrm{C}$ hot plate for 120 seconds. This voltage vs. time plot was captured by the SPA in custom mode with a 0.1 second sampling interval. 
The maximum photovoltage produced by each sample after exposure to each temperature was estimated by subtracting the average value of the two center valleys from the value of the middle peak. These maximum PM photovoltage values were then plotted using Excel to show any trends in the photovoltage due to the elevated temperatures. This is shown in Figure 78.

\section{Voltage vs. Temperature}

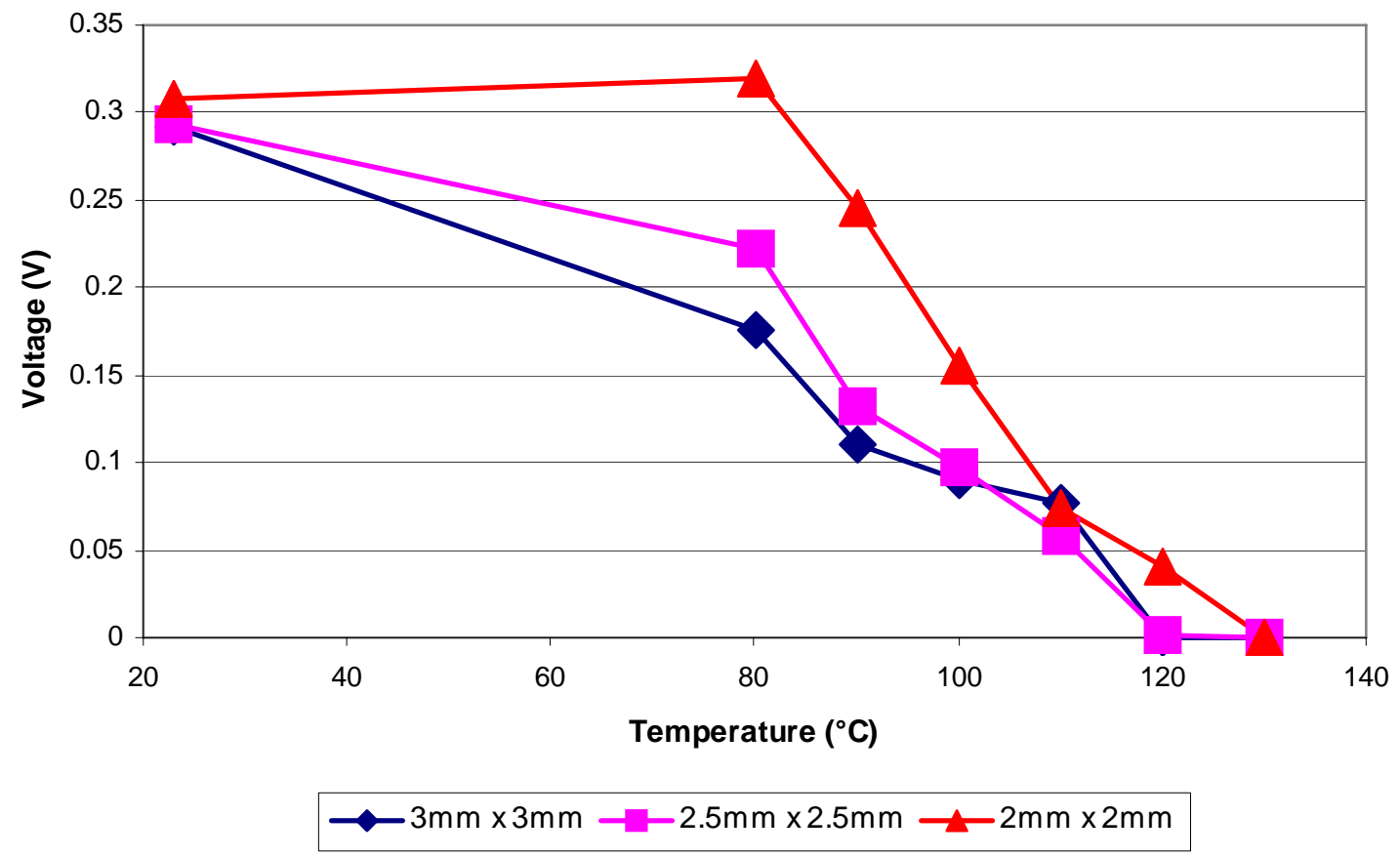

Figure 78: Correlation between maximum un-amplified PM photovoltage and temperature for each of the three patterned PM square samples.

Figure 78 shows a clear decrease in the photovoltage output of the patterned PM films as the temperature exposure was increased. The slope of the three lines after the $80^{\circ} \mathrm{C}$ voltage measurement shows that the loss of PM output is more pronounced above $80^{\circ} \mathrm{C}$. A complete loss of PM photoresponse in the $3 \mathrm{~mm}$ and $2.5 \mathrm{~mm}$ samples was observed after exposure to $120^{\circ} \mathrm{C}$. The $2 \mathrm{~mm}$ sample showed no PM photoresponse after exposure 
to $130^{\circ} \mathrm{C}$. A visible color change in the PM films from purple to clear was observed at $120^{\circ} \mathrm{C}$. This color change was irreversible, and was most likely due to the destruction of the retinal contained in the bR molecules that give PM its purple color. This retinal is required for the bR photocycle to occur, which explains why a loss of photovoltage response accompanied the loss in PM film color.

These results provide valuable information on how PM films respond to elevated temperatures. A clear decline in the photovoltage produced by the three PM films was observed when temperatures were increased to $90^{\circ} \mathrm{C}$ and above. The robust nature of PM is demonstrated by the fact that the films were able to withstand temperatures as high as $110^{\circ} \mathrm{C}$ without complete loss of function. This information is useful when designing systems incorporating PM films that are to be used in the field. This information also provides useful guidelines when developing future alterations to the PBBPT. PM films should not be exposed to temperatures exceeding $80^{\circ} \mathrm{C}$ in order to preserve the full functionality of the PM films.

\subsection{Comparison with Other Patterning Techniques}

The PBBPT has several key characteristics that make it appealing over the other major patterning techniques discussed in Chapter 1 . The first advantage of this technique is how easily it could be integrated into the large-scale production of electronic devices. Because the PBBPT takes advantage of standard photolithographic techniques, it should be much more easily integrated as a microelectronics post-fabrication process flow. The only changes the PBBPT makes to the conventional photolithography process occur after the pattern is developed, and should not add a great deal of time or complexity to the overall fabrication process. Soft lithography and FIB patterning techniques require specialized processes and tools to accomplish the patterning, while electrodeposition- 
based patterning requires compromises to be made when designing the electronic substrate to accommodate the electrodeposition process.

The PBBPT is capable of producing patterns of PM films with feature sizes comparable to both FIB and soft lithographic patterning techniques, and should greatly outperform the minimum feature size of electrodeposition-based patterning. Individual PM patches range in size from hundreds of nanometers up to microns in size. Submicron photolithographic patterns are achievable using photolithographic processing techniques. The minimum feature sizes achievable by the PBBPT as well as by soft lithography will most likely be limited, not by the patterning process capability, but by the physical size of the PM fragments or the area necessary to provide sufficient sensitivity to the antigen-antibody binding of target toxin molecules.

The PBBPT is versatile for the types of PM deposition techniques that can be used in conjunction with the patterning process. As was discussed previously, all of the major deposition techniques can be used with the PBBPT. FIB patterning can also be applied to any deposition method, but this process has proven to be impractical for large volume patterning. Electrodeposition-based patterning obviously relies on the electrodeposition process to pattern the PM film. Soft lithography is also a versatile patterning technique that can be applied to all of the major PM film deposition techniques.

The greatest competition of the PBBPT for integrating PM films into microelectronic structures is soft lithography. Both techniques offer outstanding minimum feature sizes along with flexibility in the way PM can be deposited. Both are relatively wellestablished techniques, however conventional photolithography is much more widely used, and has been studied for decades longer than soft lithography. The PBBPT is distinguished from soft lithography in the logistics of integrating the PM patterning process into a conventional microelectronics production flow. Soft lithography uses polymeric stamps that must be replaced after several uses to maintain process reliability. 
The PBBPT uses a standard photolithography photomask to produce the patterns, which can be used indefinitely. Soft lithography requires specific and costly equipment to be added to a standard photolithographic photoaligner. Alignment of a soft lithography stamp with an existing electronic structure on the substrate could also prove difficult. Unless the underlying electronic circuitry is being produced using soft lithography, PBBPT is a more practical patterning technique to integrate into a microelectronic device's fabrication process flow.

\subsection{Applications}

Although the PBBPT is being developed to allow the patterning of PM films onto an electronic substrate, this is just one possible application of this patterning technique. This process can be used to pattern PM or other biological materials onto any flat substrate. The flatness of the substrate is important because the photomask used to define the patterns will not conform to non-flat surfaces. The PBBPT provides a quick and relatively straightforward method for patterning oriented PM.

While the PBBPT has been studied and optimized for the patterning of PM films, the technique is not limited to this material. Any biological material that can withstand a photoresist stripping agent such as acetone, and can be made to adhere to a solid substrate, could be patterned using this method. Currently there is a high demand for the ability to pattern biological materials in arrays and other geometric patterns on solid substrates. The volume of work being published relating to the patterning of various biological materials indicates the importance of patterning for various avenues of research. The PBBPT will provide researchers with an alternative method to soft lithography that is capable of patterning large quantities of biological materials without the need to cast polymeric stamps. The PBBPT is a novel approach to patterning biological materials that could facilitate research in a wide variety of different fields. 


\subsection{Integration of PM films with MOSFET Transistors}

The main goal of this research was to successfully integrate patterned PM films with microelectronic devices. The development of the PBBPT allowed for PM films to be oriented, deposited and patterned onto a flat substrate without compromising the photoelectric functionality of the film. The final step towards the end goal of this project was to integrate a PM film patterned using the PBBPT with some sort of microelectronic device. The device needed to be sensitive to changes in gate voltage, which would be provided by the PM film. MOSFET devices met all of the criteria, and were selected to interface with the patterned PM films.

One reason that MOSFET devices were selected was because of their predominant use for microelectronic circuitry. Superior scalability and low power consumption are two of the reasons that MOSFET usage has become widespread. These devices are widely used and well characterized. Previous work by Bhattacharya et al. [28] demonstrated the successful functionalization of the gate of a Ga-As field effect transistor with un-patterned PM. This result confirmed the ability of a PM film to activate a field effect transistor.

A six inch test wafer containing both PMOS and NMOS FETs was obtained from a major semiconductor manufacturer under a Non-Disclosure Agreement. Each device on the wafer was individually addressable through contact pads leading to the source, drain, gate and body of the transistor. These pads could be contacted using micromanipulator probes to modulate a single device. PMOS FETs were used exclusively in the studies discussed below. Because of confidentiality restrictions, no further details or images of individual devices will be presented.

The test wafer contained a large number ( $>50$ ) of identical blocks. Each block contained identical arrays of devices. Each block was split into smaller sections, each 
containing a particular type of device. One of these sections contained 21 individual PMOS transistors that would be used in the integration experiments described below. The first step was to dice the wafer to allow for multiple experiments to be conducted using the individual blocks of transistor arrays. The wafer was successfully diced using a Micro Automation Model 1100 Micro Dicing Saw.

\section{Integration of Patterned PM films onto MOSFET Substrates}

The initial attempts to integrate PM films with the MOSFET arrays involved deposition and patterning of the PM film directly onto the MOSFET substrate. To accomplish this task, several masks were designed that allowed for PM sensing sites to be placed either directly on top of the gate contact pads, or placed remotely and electrically connected to the individual transistor gate pads. Unfortunately, no data on the size or spacing of the individual transistor contact pads was provided by the wafer manufacturer. To design a mask, the locations of each contact pad in the PMOS device array were measured.

Digital images of the sample were taken using an optical microscope coupled to a Nikon D200 digital SLR camera. Images were also taken of a scale bar, which was used to determine the length represented by each pixel in the captured images. The images were analyzed using software which displayed the number of pixels between points in the images. The number of pixels between points was multiplied by the distance represented by each pixel to obtain the distance between the points. Using this tool, the contact pad size and locations were obtained.

After the dimensions and locations of the PMOS transistor array pads were obtained, several lithographic masks were designed using Cadence. One mask allowed for PM films to be deposited directly onto the gate pad of each individual transistor in the array. Other mask designs allowed for remote PM sensing sites ranging in size from $250 \mu \mathrm{m}$ 
squares up to $4000 \mu \mathrm{m}$ squares. These sensing sites were electrically connected to individual transistor gate pads by $30 \mu \mathrm{m}$ wide lines.

After the mask design was completed, a transparency mask was fabricated in-house. The transparency mask was a relatively low quality lithographic mask that was used to prove the successful integration of patterned PM films onto the MOSFET substrate. A higher quality mask with smaller line widths would be designed and fabricated off-site if the basic process proved successful.

A MOSFET array was lithographically patterned using the same protocol used to pattern ITO coated glass slides in Section 4.4. The transparency mask described above defined lines from individual transistor gate pads out to $4 \mathrm{~mm}$ square areas that acted as PM sensing sites. Following lithographic patterning, the substrate was coated with $20 \mathrm{~nm}$ of gold. A schematic representation of the MOSFET functionalization scheme is shown in Figure 79. This is a general representation of the functionalization scheme, and is not drawn to scale. 


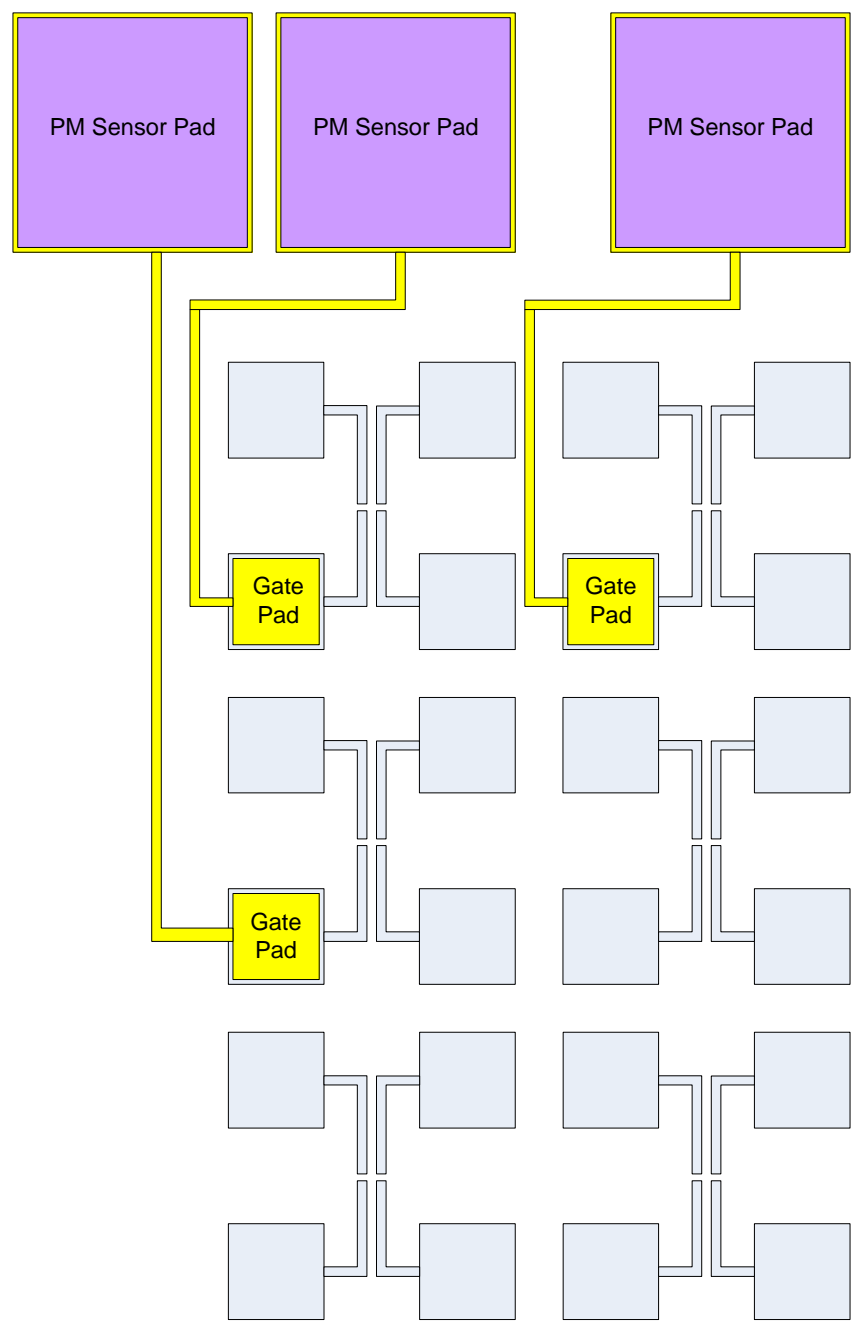

Figure 79: Schematic representation of the integration of PM films with a PMOS array using the PBBPT.

After the gold deposition, electrodeposition was performed over the large PM sensing sites defined by lithography. Two $15 \mu \mathrm{l}$ drops of suspended PM (11.6 mg/ml) were placed over the PM receptor sites on the MOSFET substrate. The two plates were separated by a $1.2 \mathrm{~mm}$ spacer, and $4.0 \mathrm{~V}$ was applied for 40 seconds. The PM film was allowed to dry under ambient conditions. After the sample had dried, it was placed in an acetone sonication bath for 7 minutes. The PM film covered the large sensing pads, as well as some of the lines and transistor gate contact pads. 
After the acetone sonication, the sample was placed on a Micromanipulator probe station. Tri-axial probes were brought into contact with the source, drain and body contacts of a single PMOS transistor. The device dimensions were $1.35 \mu \mathrm{m} \times 0.81 \mu \mathrm{m}$ (gate width $\mathrm{x}$ gate length). The transistor gate pad was functionalized by a patterned PM film. The tri-axial probes input to a Keithley 4200 Semiconductor Parametric Analyzer (SPA). The gate and body probes were set to common, and 2.0 volts was applied to the source to provide a source-drain bias. Measurements were taken in sampling mode. The SPA was equipped with a pre-amplifier that could accommodate a single tri-axial probe. The pre-amplifier was not used during this experiment.

A broad spectrum xenon light source was placed $25 \mathrm{~mm}$ away from the substrate, directly over the patterned PM sensing sites. The light was modulated by manually turning the power supply on and off as needed. A typical response of the PM functionalized MOSFET system is shown in Figure 80. 


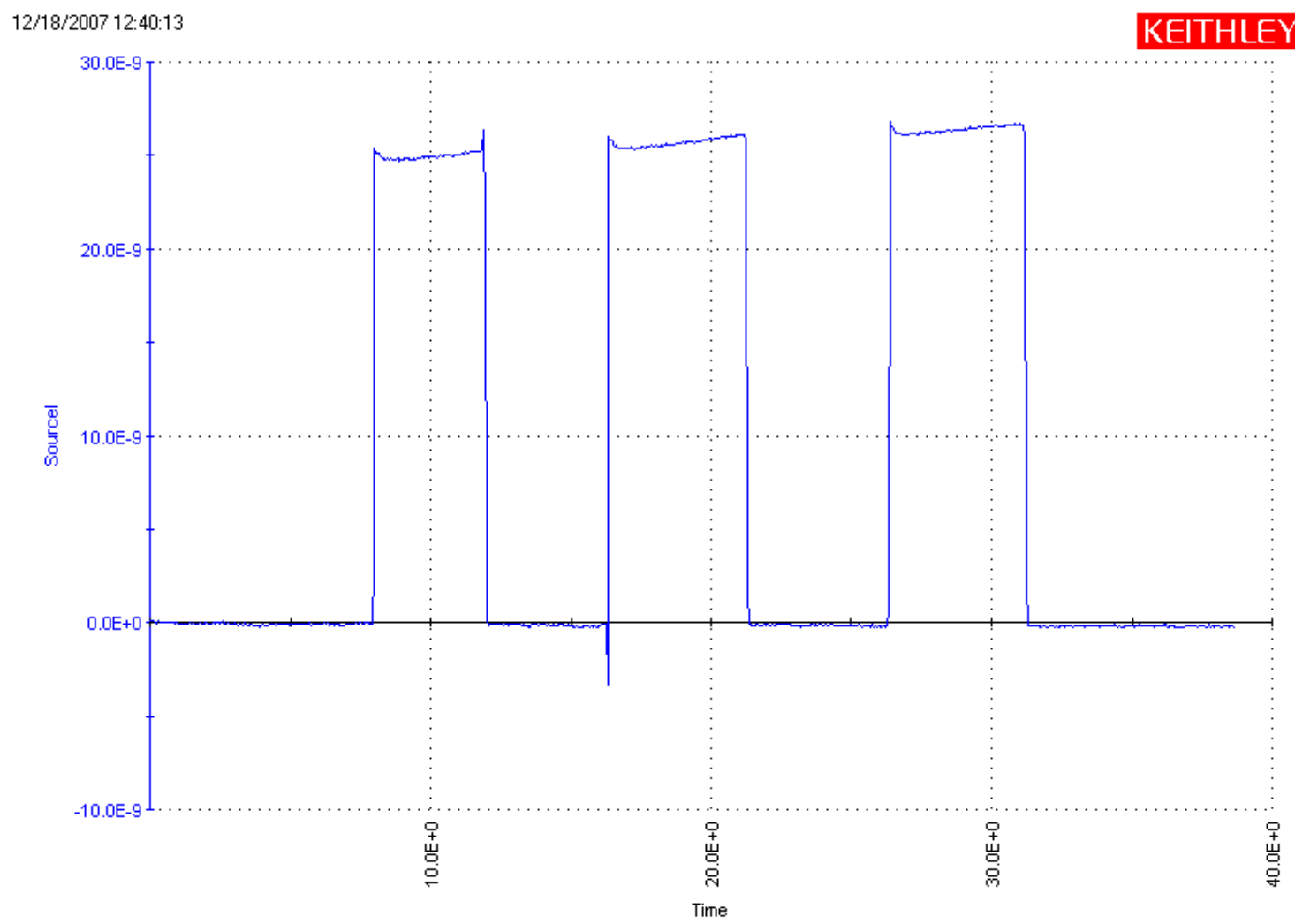

Figure 80: Source current vs. time plot due to xenon light modulation of a PMOS transistor $(1.35 \mu \mathrm{m} \mathrm{x}$ $0.81 \mu \mathrm{m}$ ) functionalized with a patterned PM film. Source-drain bias $=2.0 \mathrm{~V}$, Body Bias $=0 \mathrm{~V}$, Sampling mode, 3.0 volts to xenon light.

When the light was off, the source current was zero. Upon illumination, an immediate jump to $\sim 25 \mathrm{nA}$ seen. This was an encouraging first result, indicating that the system responded to a change in lighting conditions. To rule out the effects of any local electric field produced by the light, a resistor of similar resistance was attached to the power supply. Figure 81 shows the source current versus time plot taken while the power to the resistor was modulated. When power was run through the resistor, no change in the source current of the transistor was seen. 


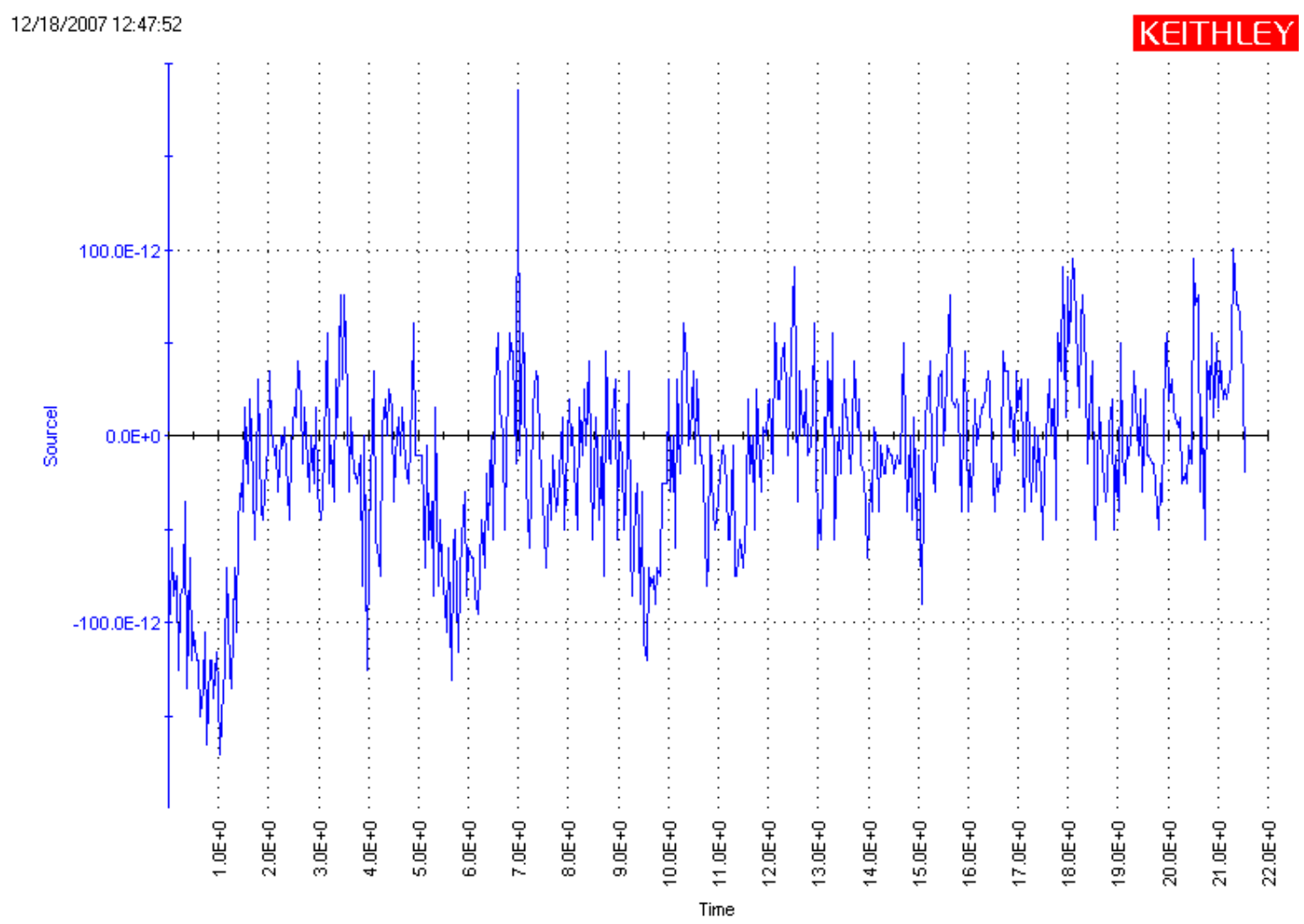

Figure 81: Source current vs. time plot due to resistor modulation of a PMOS transistor $(1.35 \mu \mathrm{m} \times 0.81$ $\mu \mathrm{m}$ ) functionalized with a patterned PM film. Source-drain bias $=2.0 \mathrm{~V}$, Body Bias $=0 \mathrm{~V}$, Sampling mode, 3.0 volts to xenon light.

After it was established that the transistor response was due to light as opposed to electrical noise induced by the lighting system, a third source current versus time measurement was taken. The PM functionalized MOSFET sample was removed, and an un-coated sample was put in its place. The same PMOS transistor $(1.35 \mu \mathrm{m}$ x $0.81 \mu \mathrm{m})$ on the un-coated sample was probed through the SPA using the same transistor operating conditions. The same lighting conditions were used to illuminate the bare transistors. The result of this experiment can be seen in Figure 82. 


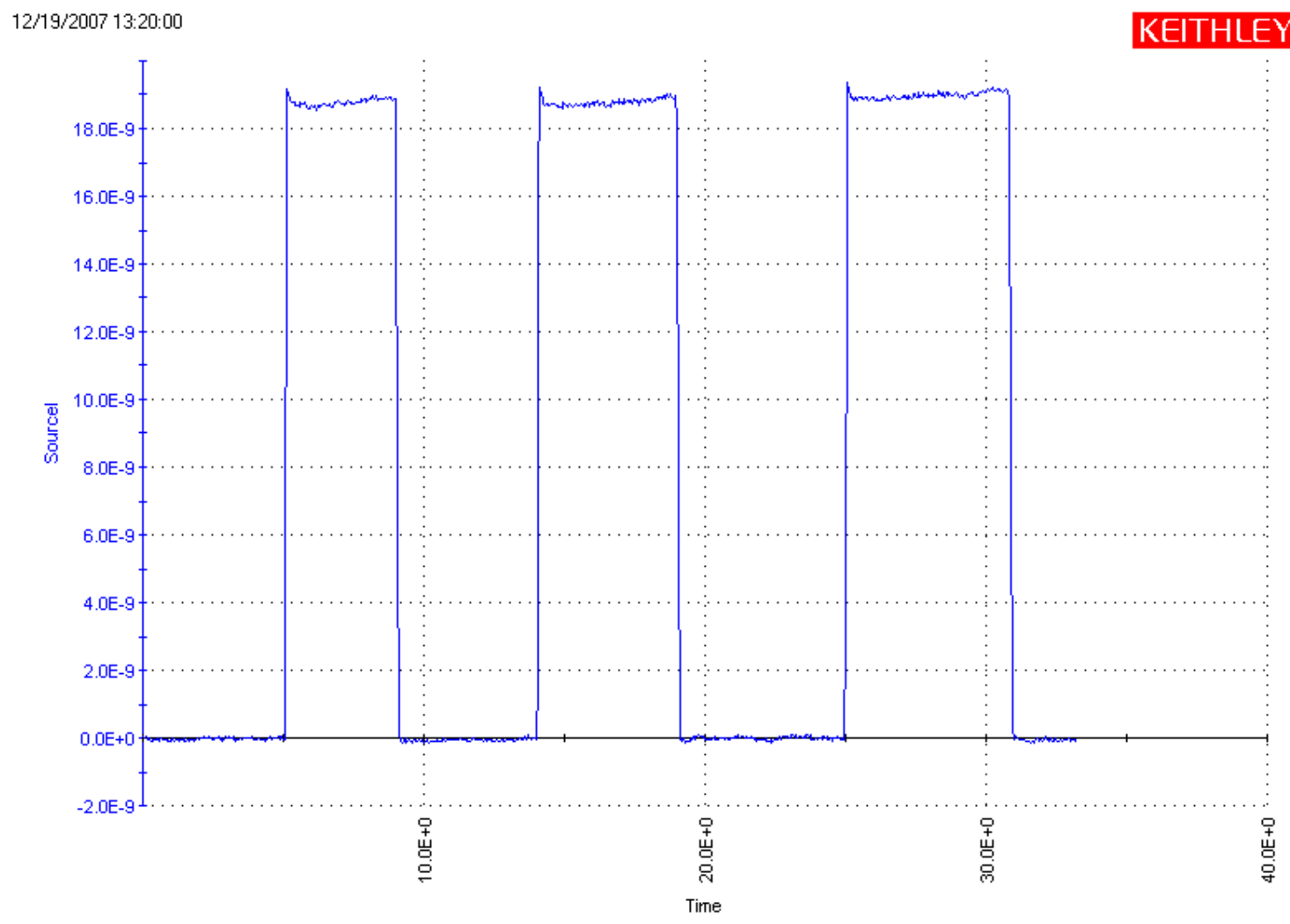

Figure 82: Source current vs. time plot due to xenon light modulation of a non-functionalized PMOS transistor $(1.35 \mu \mathrm{m} \times 0.81 \mu \mathrm{m})$. Source-drain bias $=2.0 \mathrm{~V}$, Body Bias $=0 \mathrm{~V}$, Sampling mode, 3.0 volts to xenon light.

Figure 82 demonstrated that the PMOS transistor responded to light without any functionalization. The responses of the functionalized and non-functionalized samples were very similar both in shape and in magnitude. The small difference in magnitude between the two was most likely caused by the light not being positioned in exactly the same place on the two samples.

This result was a major setback in the attempt to integrate patterned PM films with a MOSFET. Because the individual transistors were extremely sensitive to light, it would be impossible to determine with confidence if the PM films were contributing to the response of the transistor to light. The responsiveness of the non-functionalized 
transistors to light was most likely due to the photoelectric effect, which occurs when the energy from a photon of light is absorbed by an atom on the surface of the exposed substrate, resulting in an electron being released by the atom. MOSFETs function based on the availability of charge carriers across the semiconducting gap between the source and drain. Electron removal from the substrate due to light can modulate the transistor without any applied gate voltage.

\subsection{Eliminating Light Induced Transistor Activation}

One way to control the photoelectric effect in MOSFETs is to alter the design and fabrication of the devices. Because completed devices were received, no modifications to the actual devices could be made. The only other alternative to eliminate the photoresponse of the transistors was to shield the transistors from the light. The first attempt was to deposit a chrome film over the active area of the transistors. To accomplish this, a transparency mask was designed and fabricated which allowed for films to be deposited in the areas between the four contact pads. A schematic representation of this patterning process can be seen in Figure 83. 
MOSFET Transistor Array

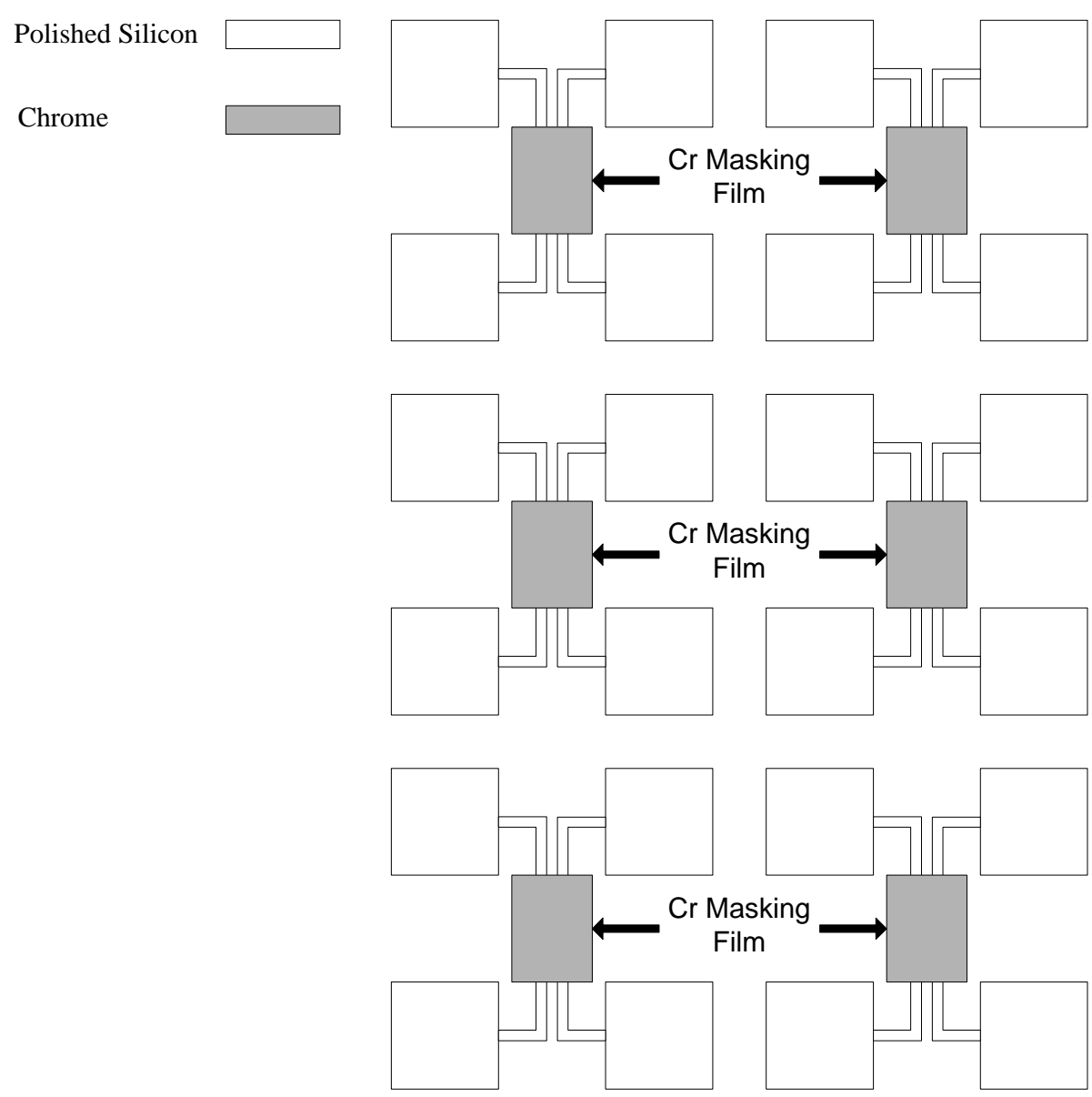

Figure 83: Schematic representation of the chrome film masking of PMOS transistor active areas.

A MOSFET substrate was lithographically patterned using the mask. The photolithographic protocol described in Section 4.4 was used. The substrate was sputter coated with $150 \mathrm{~nm}$ of $\mathrm{Cr}$, and a 90 second acetone sonication bath was used to strip the photoresist and lift off the unwanted Cr film. The responses of individual transistors to light were then observed using the probe station and SPA.

To begin the experiment, probes were used to connect the source, drain, gate, and body pads of a PMOS transistor $(4.05 \mu \mathrm{m} \times 0.81 \mu \mathrm{m})$ to the SPA through tri-axial cables. A drain voltage was swept from 0 to -5.0 volts, while the gate voltage was stepped from 
0 to -1.0 volts. A body bias of -0.3 volts was also applied. An increase in drain current as the gate voltage increased indicated that the transistor was functioning properly. After transistor functionality was confirmed, the gate probe was removed and the source, drain and body terminals were connected through tri-axial cables and probes to the SPA. A voltage of -2.0 volts was applied to the drain of the device. A voltage of -0.3 volts was applied to the body terminal of the device. Applying a bias of the correct polarity to the body of the transistor decreases the threshold gate voltage required to activate the transistor. By providing a body bias to the transistor, its sensitivity to gate voltages or light was increased. This was done to ensure that the photo-induced transistor response had been eliminated.

An incandescent bulb run through a fiber optic line to the probe station microscope provided illumination to the sample. The light was passed through a $100 \mathrm{X}$ microscope objective, resulting in a fine point of light that could be moved across the sample via the microscope stage controls. The intensity of the light could be modulated. The SPA was set to sampling mode, and drain current versus time data was taken. After the measurement was started, the microscope light was pulsed directly on top of the PMOS transistor array. The response of the single probed transistor is shown in Figure 84. 


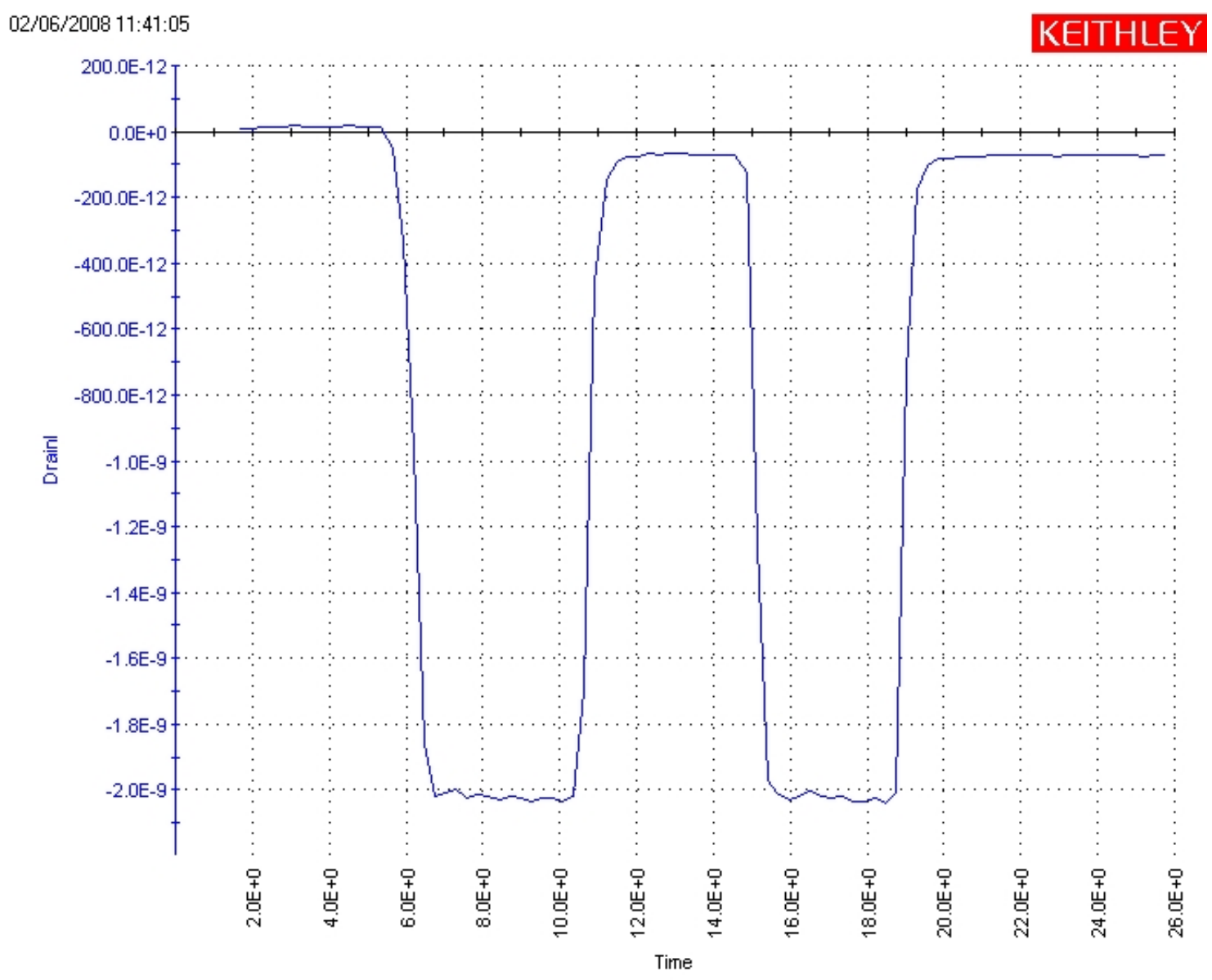

Figure 84: Drain current vs. time plot of a PMOS transistor $(4.05 \mu \mathrm{m} \times 0.81 \mu \mathrm{m})$ exposed to two pulses of incandescent light. The light pulses resulted in a negative gate current response.

The results of this experiment indicated that coating the active area of each individual transistor was not effective in eliminating the photo-induced response of the PMOS transistors. A clear negative response to illumination was observed, and can be seen in Figure 84. If the $\mathrm{Cr}$ film had been effective at shielding the device from the effects of the light, no change in drain current would have been observed during illumination of the transistor array.

Several additional studies were performed that investigated moving the PM sensing site as far away from the functionalized PMOS transistors as possible. These studies 
showed the same end results as the study above, that the transistors were still sensitive to the light used to activate the PM film. All attempts to functionalize the MOSFET substrate directly with patterned PM films were unsuccessful. Any electrical responses produced by the PM films could not be differentiated from the response of the transistors to light.

The failure to directly functionalize the MOSFET substrate using the PBBPT process was due to the wafer used in these experiments. The issue of transistor activation due to light is a problem that can be solved in the device fabrication process. Passivating layers that shield the transistors from all light are routinely applied to transistor arrays to improve functionality. Because the devices used in these experiments were produced for testing purposes, no light shielding coatings were applied. To avoid the transistor light activation issue and still utilize the MOSFET test arrays, an indirect functionalization scheme was developed.

\subsection{Indirect Functionalization of MOSFETs Using Patterned PM Films}

Direct functionalization of the MOSFET substrate failed because all attempts to shield the substrate from the light used to activate the PM films were unsuccessful. One solution to that problem was to move the patterned PM film off of the MOSFET substrate. This allowed for the MOSFET substrate to be completely isolated from the illumination of the PM film. To integrate the patterned PM films with the PMOS transistors, a method was developed for transferring the signal from the PM to the gate pad of the transistor.

The PBBPT process was used to produce 8 patterned PM films on ITO coated glass slides. The PBBPT procedure discussed in Section 4.4 was used to pattern the substrates and deposit the PM films. After PM deposition, the 8 samples were sputter coated with $\sim 7 \mathrm{~nm}$ of gold. Each sample was subjected to an 8 minute acetone sonication bath. The 
result was square patterned PM films with a thin top coating of gold over the PM films only.

After the patterned PM films were fabricated, the $3.5 \mathrm{~mm}$ square film was connected to the gate of a PMOS transistor $(67.5 \mu \mathrm{m} \times 1.62 \mu \mathrm{m})$ using co-axial probes. A co-axial probe was brought into contact with the ITO substrate on which the PM film was deposited. The probe was connected to a second co-axial probe through a co-axial coupler. This second co-axial probe was brought into contact with the gate pad of the PMOS transistor. Tri-axial probes were used to contact the transistor's source, drain, and body pads. The fourth tri-axial probe was used to contact the thin gold film covering the top of the PM film. All four tri-axial probes were connected to the SPA. A schematic representation of this experimental setup can be seen in Figure 85. 


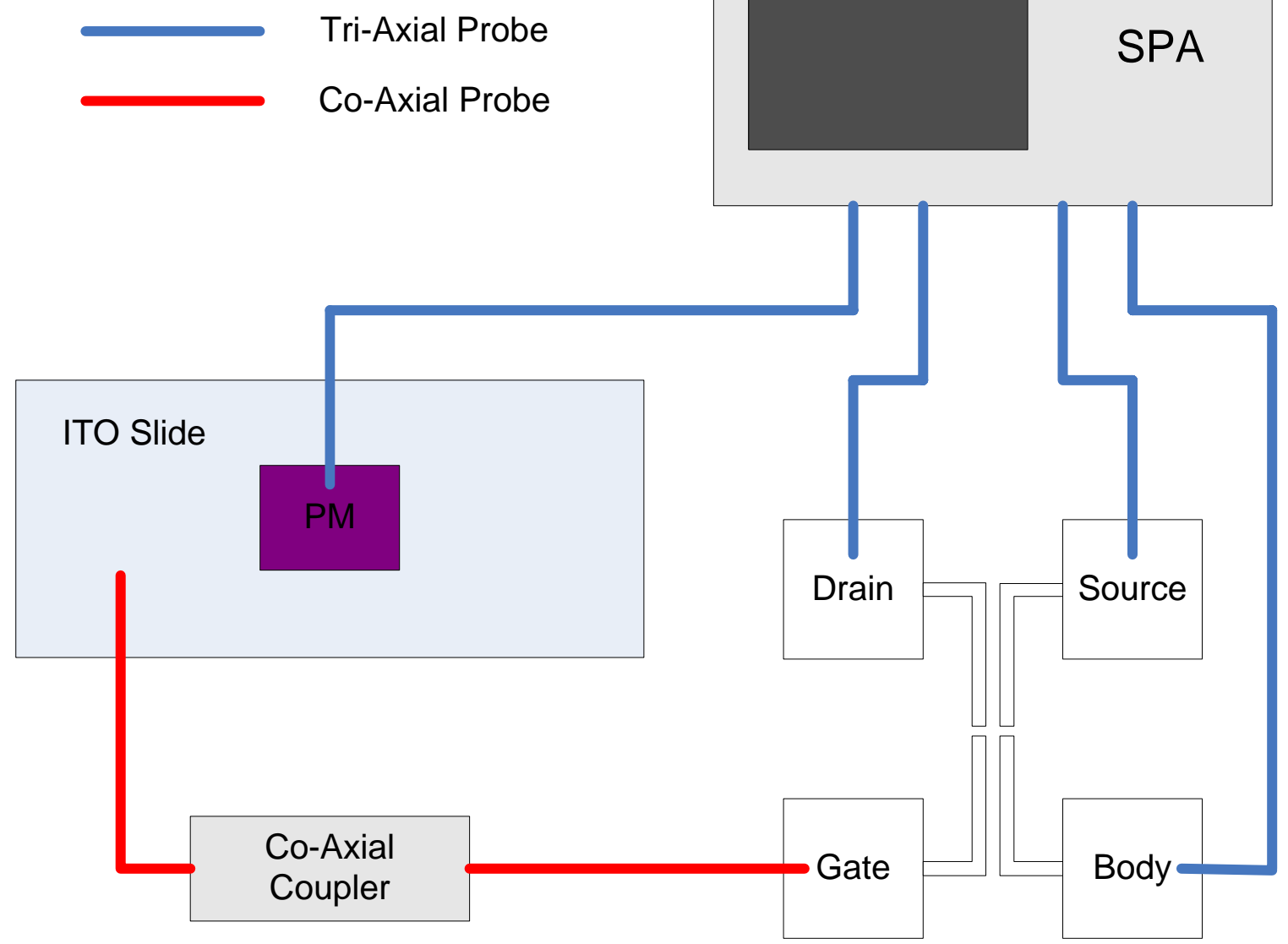

Figure 85: Schematic representation of the measurement setup used for the remote integration of a patterned PM film with a PMOS transistor.

To run the test, -3.0 volts was applied to the transistor drain. A body bias of -0.5 volts was applied to the body pad of the transistor. The source pad and the gold film on top of the PM were both referenced to ground. The SPA was run in sampling mode, collecting drain current versus time. The PM sample was located $150 \mathrm{~mm}$ from the MOSFET substrate. The LED array discussed in Section 4.4 was used to activate the PM film. The LED array was located $50 \mathrm{~mm}$ from the PM film, and provided $6.804 \mathrm{~mW} / \mathrm{cm}^{2}$ of optical power. A solid divider separated the PM film sample from the MOSFET substrate in order to shield the substrate from the light. The light was modulated manually through the power supply. 


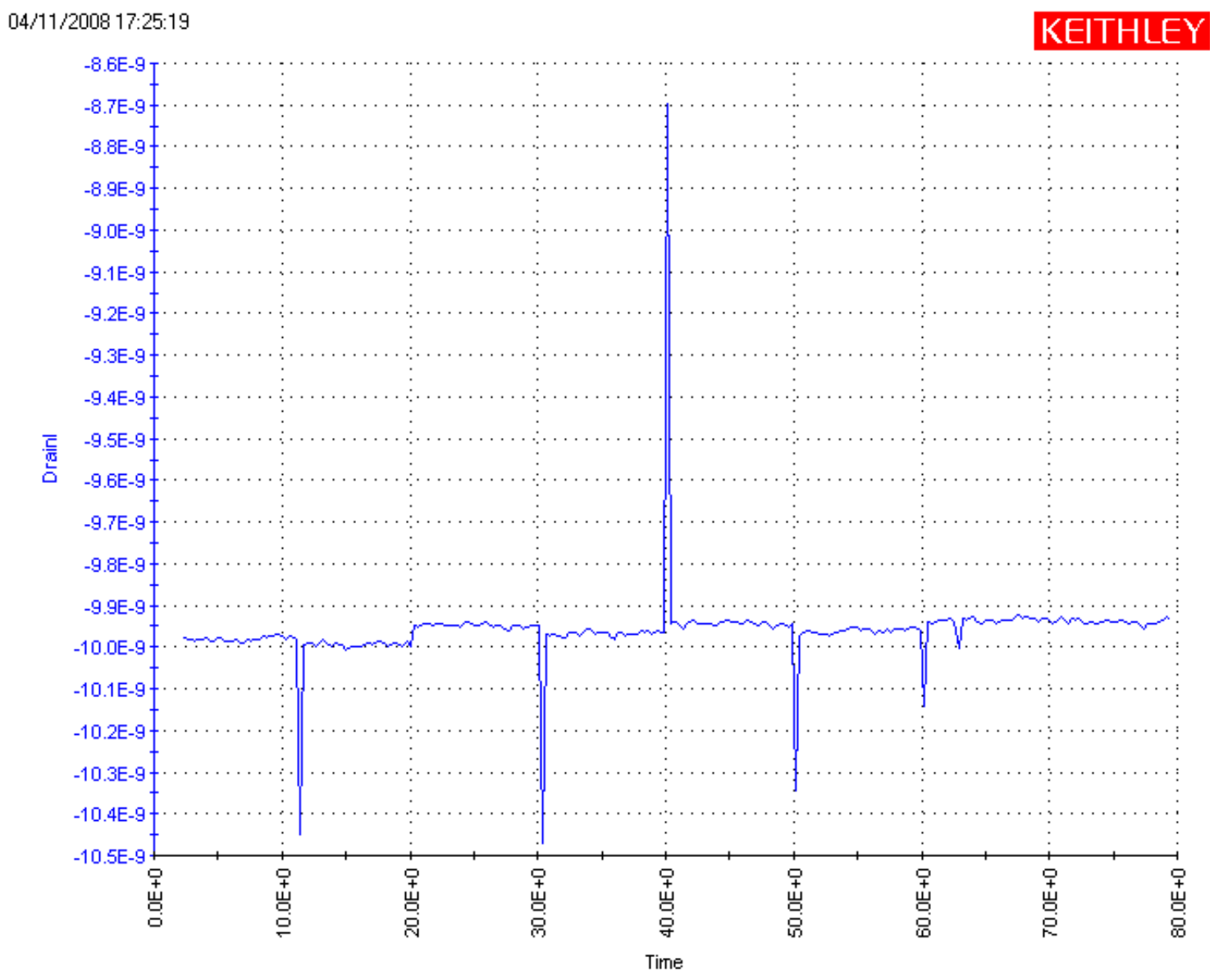

Figure 86: PMOS transistor (67.5 $\mu \mathrm{m} \times 1.62 \mu \mathrm{m}$ ) response (drain current vs. time) to remote activation by a patterned PM film. Light was turned on at $~ 11$ seconds and cycled between on and off every 10 seconds. Square wave response is indicative of light-induced photoelectric transistor response.

Figure 86 shows the transistor response to the light being turned on at 11 seconds and modulated on or off every 10 seconds. The sharp spikes occurred when the LED power supply was turned on or off. The cause of these sharp spikes in drain current are not fully understood at this time. One possibility is that leakage off of the gate of the device is causing the quick spikes in drain current. Each PMOS transistor gate is equipped with a gate protection diode, which may contribute to the behavior seen above. A small decrease in the gate current was observed when the light source was on. Based 
on the shape and magnitude of this curve, it is likely that this response was due to a small amount of light from the LEDs reaching the transistor.

To confirm that the photoresponse of the PM film was being carried through the coaxial cables to the gate of the transistor, a second measurement was taken. To perform this measurement, the co-axial probe contacting the gate of the transistor was raised into the air. The tri-axial probe from the drain was raised, and brought into contact with the co-axial probe. A two-probe test was then run in sampling mode with no voltage supplied to either probe by the SPA. The LED light source was cycled on twice, and the resulting voltage produced by the PM film is shown in Figure 87. 


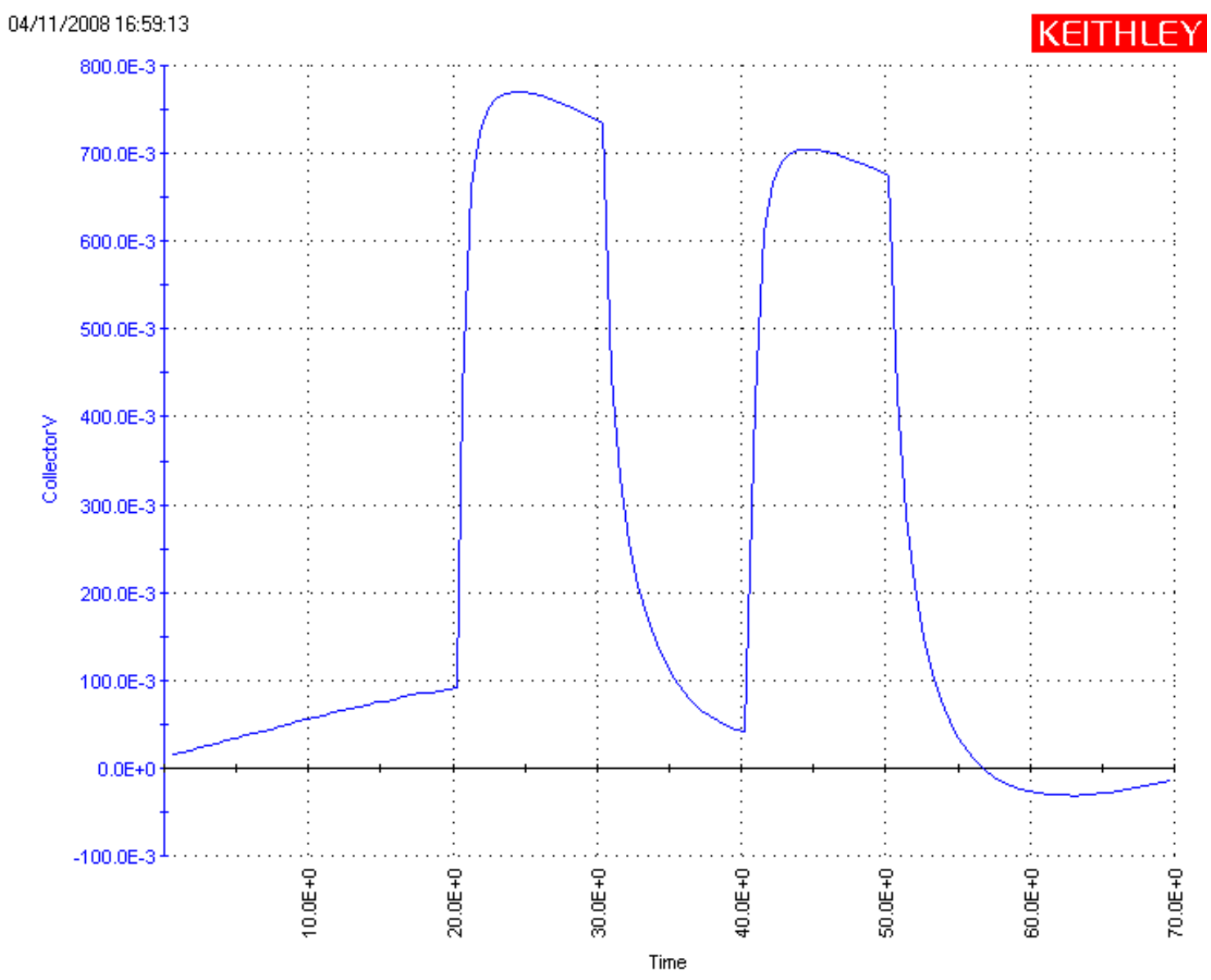

Figure 87: Voltage response of patterned PM film to an LED light source. The PM film signal was run through two co-axial probes.

The patterned PM film produced a voltage of $\sim 700 \mathrm{mV}$ when exposed to the LED lighting. When a voltage of $700 \mathrm{mV}$ is applied to the PMOS transistor under the same conditions described in the previous study, a large drain current (-100's of mA) is produced. This fact indicated that the PM signal was not being effectively transferred to the transistor gate. This experiment confirmed that the signal is not lost in the probes used to transfer the signal from the PM to the transistor. One possible cause for why the signal produced by the PM film was not activating the PMOS transistor was an impedance mismatch between the PM film and the transistor. The spikes seen in Figure 86 help to support the theory that an impedance mismatch is present in this experimental 
setup. These transistor current spikes may indicate that the voltage produced by the PM film is activating the transistor for a very short time, after which the voltage is bled away due to the impedance mismatch between the PM film and the transistor. These results prompted further study into the impedance matching of the PM film and the PMOS transistor.

\subsection{Remote Integration of Patterned PM Films with MOSFETs Through Impedance Matching}

An impedance mismatch occurs when the output impedance of a source does not match the input impedance of a device. A large mismatch between the impedance of the source (the PM film) and the impedance of the device being loaded (the PMOS transistor) can result in a large loss of input signal at the device. This phenomenon can be represented mathematically using Equation 2, where $\mathrm{V}_{\text {gate }}$ is the voltage seen at the transistor gate, $\mathrm{V}_{\mathrm{PM}}$ is the voltage supplied by the PM film, $\mathrm{I}_{\text {gate }}$ is the impedance of the transistor gate, and $\mathrm{I}_{\mathrm{PM}}$ is the impedance of the PM film [33].

$$
\text { Equation 2: } V_{\text {gate }}=V_{P M} \frac{I_{\text {gate }}}{I_{\text {gate }}+I_{P M}}
$$

In order to minimize the signal loss due to an impedance mismatch, $I_{\text {gate }}$ should be much larger than $\mathrm{I}_{\mathrm{PM}}$. Unfortunately, PM films are highly resistive, while PMOS transistors tend to have lower resistivities. In this case, $\mathrm{I}_{\mathrm{PM}}$ is much larger than $\mathrm{I}_{\text {gate, }}$, which makes the voltage at the gate extremely small. In order to overcome this phenomenon, impedance matching was used. Several different methods can be used to match the impedance of the two components, allowing the signal to pass from the PM film to the PMOS transistor. One method involves placing an operational amplifier between the source (PM) and the device (PMOS transistor). The operational amplifier 
isolates the source from the device, so that any mismatch between the two does not impact the transmission of the signal.

To solve the problem of impedance mismatch, a high impedance operational amplifier with a $2 \mathrm{X}$ gain was used to provide impedance matching between the highly resistive PM film and the less resistive PMOS transistor. The patterned PM samples tested in Section 5.3 were also used in this study. Instead of contacting the PM sample using probes, the $3.5 \mathrm{~mm}$ patterned square PM sample was placed in the mounting chuck, which was then placed inside the grounding box discussed in Section 4.4. This setup ran the electrical connections for the top and bottom of the PM film to a male co-axial connector. The male co-axial output from the grounding box was coupled to the co-axial input of the operational amplifier. A schematic representation of the experimental setup is shown in Figure 88. 


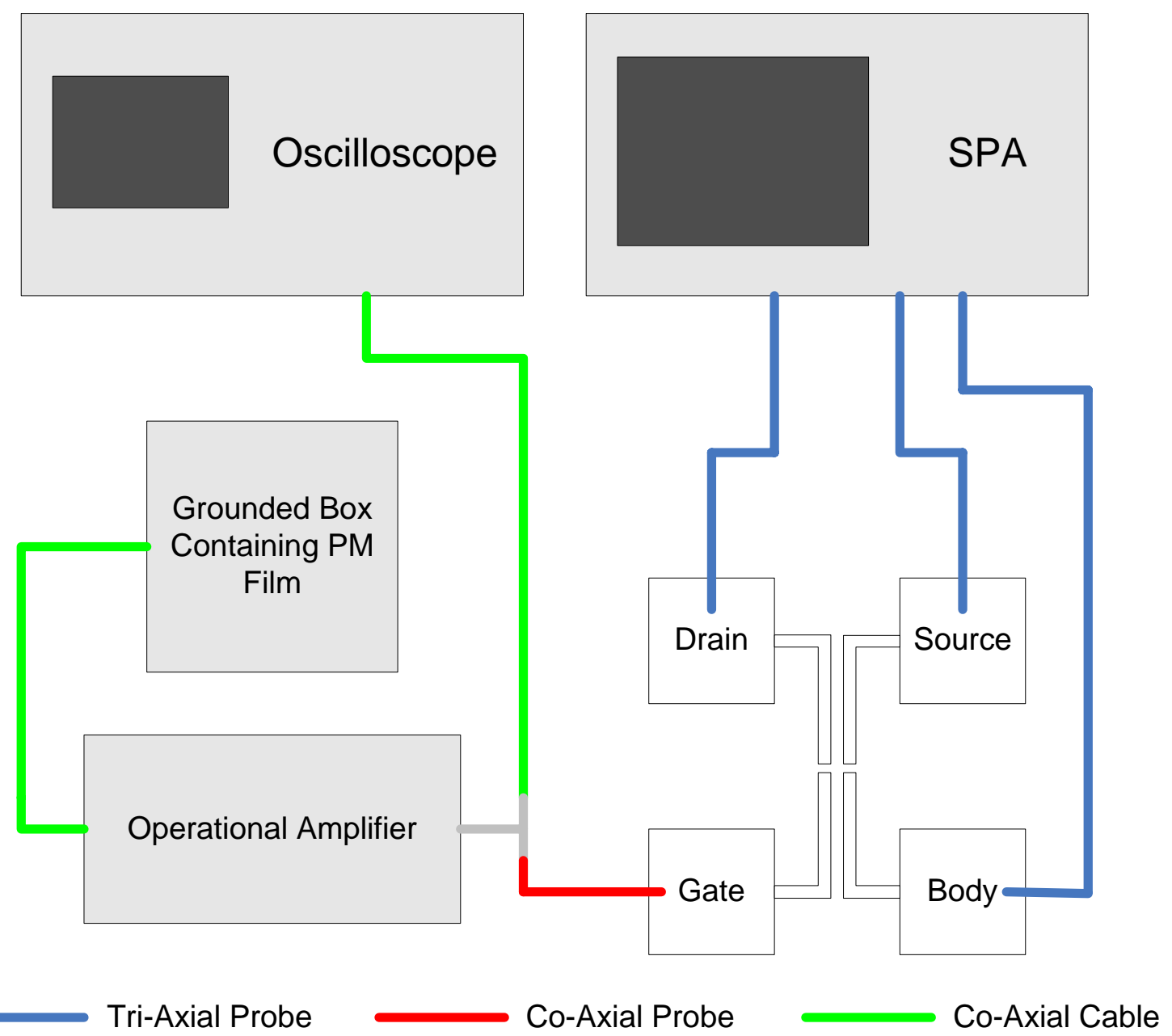

Figure 88: Schematic representation of the measurement setup used for the remote integration of a patterned PM film with a PMOS transistor using impedance matching.

The output from the operational amplifier was split using a T-connector. One of the output lines went to an Agillent 54622A oscilloscope, while the other was coupled to a co-axial probe on the probe station. The co-axial probe was brought into contact with the PMOS transistor $(67.5 \mu \mathrm{m}$ x $67.5 \mu \mathrm{m})$ gate pad. The source, drain, and body pads were connected to the SPA using tri-axial probes. This measurement setup allowed for PM voltage measurements to be captured by the oscilloscope, while drain current measurements were simultaneously captured using the SPA. The LED array discussed in Section 4.4 was again used to provide illumination to the PM film. A pulsing circuit controlled the flash rate of the LED array. The PM sample and LED array were placed 
on a table under the probe station to shield the transistors from the light used to activate the transistors.

After configuring the experimental setup, the LED light source was powered through the power supply circuit and a constant pulse rate with a cycle time of 28 seconds and a duty cycle of $50 \%$ was provided by the pulsing circuit. The optical power provided to the PM film was measured at $1.302 \mathrm{~mW} / \mathrm{cm}^{2}$. The oscilloscope took real time measurements of the PM photovoltage in DC mode. The PMOS transistor was provided with a drain voltage of -2.0 volts and a body bias of -0.4 volts. The source pad of the transistor was set to common. All lighting, other than measurement displays and the LED light source, were turned off during the measurements. Figure 89 shows the voltage response of the PM film to the pulsed LED light, while Figure 90 shows the PMOS transistor response to the photovoltage provided by the PM film to the gate of the transistor.

PM Photovoltage

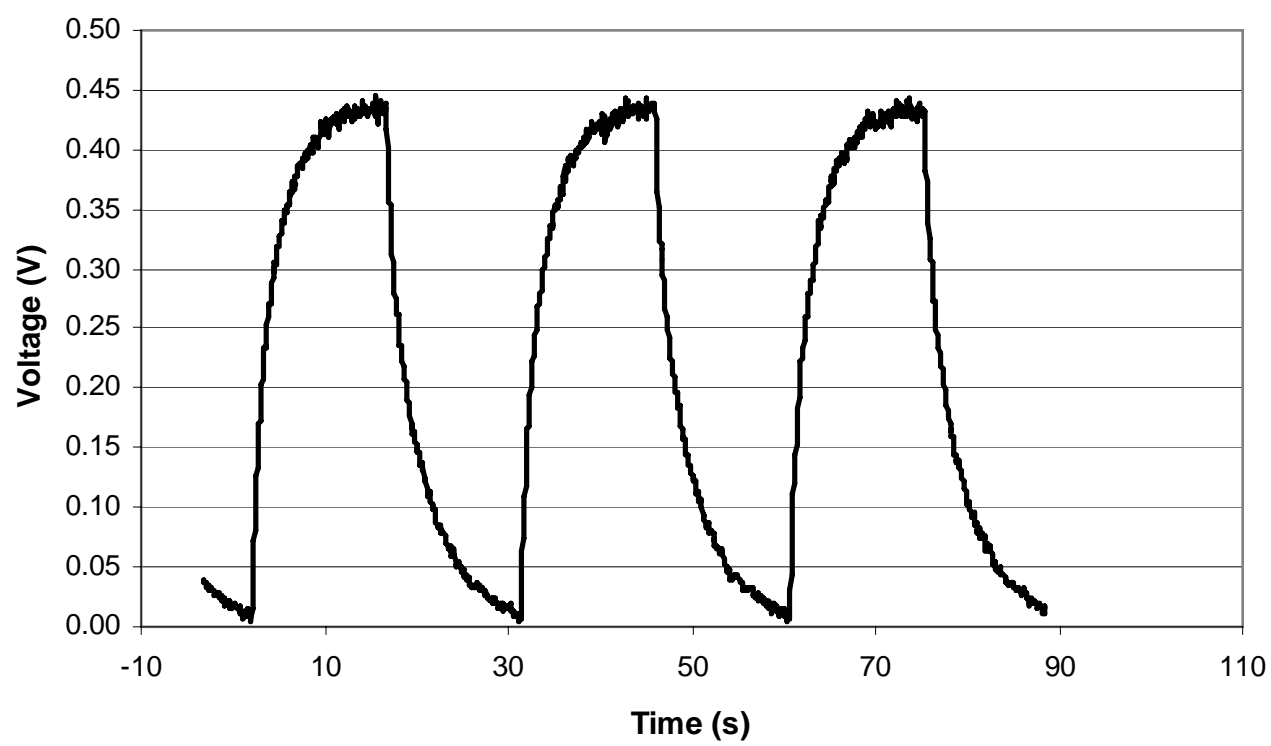

Figure 89: PM photovoltage due to pulsed LED light. $\quad$ LED optical power $=1.302 \mathrm{~mW} / \mathrm{cm}^{2}$, light cycle time $=28$ seconds, duty cycle $=50 \%$. 


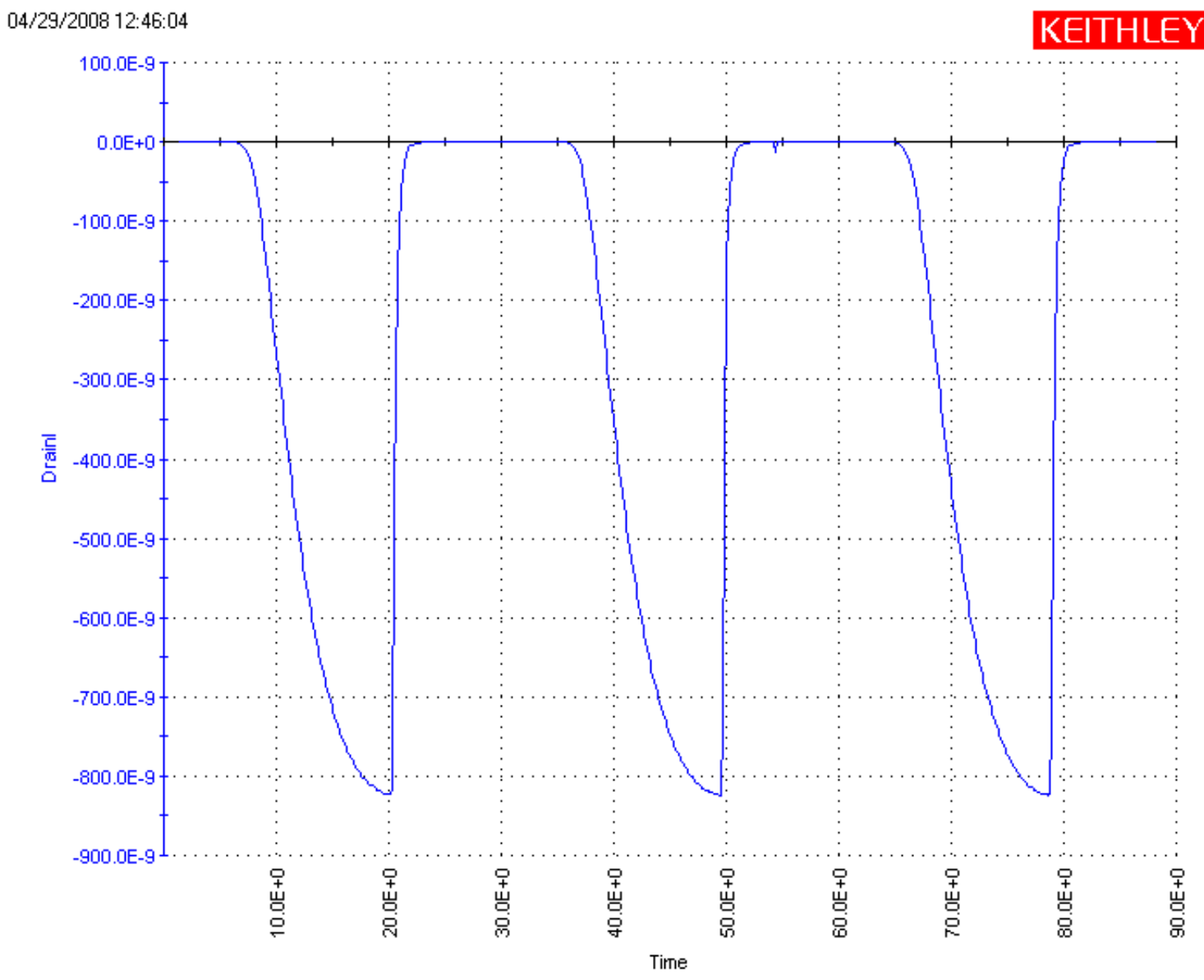

Figure 90: PMOS transistor ( $67.5 \mu \mathrm{m} \times 67.5 \mu \mathrm{m}$ ) response (drain current vs. time) to remote patterned PM film photovoltage activation. A $2 \mathrm{X}$ gain was applied to the PM photovoltage by the operational amplifier. Source-drain bias $=-2.0 \mathrm{~V}$, body bias $=-0.4 \mathrm{~V}$. Time divisions $=10$ seconds, drain current divisions $=1$ $\mu \mathrm{A}$.

The maximum photovoltage produced by the patterned PM film under the lighting conditions described above was approximately $430 \mathrm{mV}$. This voltage resulted in over $800 \mathrm{nA}$ of drain current in the PMOS transistor. This transistor response was five orders of magnitude above the noise level for this test setup, and indicated that the transistor was being activated by the PM film. The shape of the transistor response mirrors the shape of the PM photovoltage measured by the oscilloscope, which is further evidence that the 
transistor activation is due to the PM photovoltage supplied to the transistor gate. To rule out electrical noise or stray light as sources for the PMOS activation, a measurement was taken with the PM film shielded from the light. The result was no measured photovoltage and no transistor activation.

A separate experiment was run to confirm that the shape of the PMOS transistor response was due to the PM photovoltage and not the transistor or measurement equipment. Square waves from An Agilent 33250A function generator was run through the $2 \mathrm{X}$ gain op-amp to the gate pad of a PMOS transistor $(67.5 \mu \mathrm{m} \times 0.81 \mu \mathrm{m})$ using a co-axial probe on the probe station. Tri-axial probes were used to connect the source and drain pads of the transistor to the SPA. The body pad of the transistor was not contacted for this experiment. A voltage of -2.0 volts was applied to the drain of the transistor, and the source of the transistor was set to ground. The drain probe was run through the SPA's pre-amplifier to increase the sensitivity of the drain current measurements. A gate current versus time measurement was taken in sampling mode using the SPA while a 30 $\mathrm{mV}$ peak to valley square wave with a frequency of $0.1 \mathrm{~Hz}$ and a duty cycle of $50 \%$ was sent to the transistor gate. The response of the transistor to the square wave gate input is shown in Figure 91. 


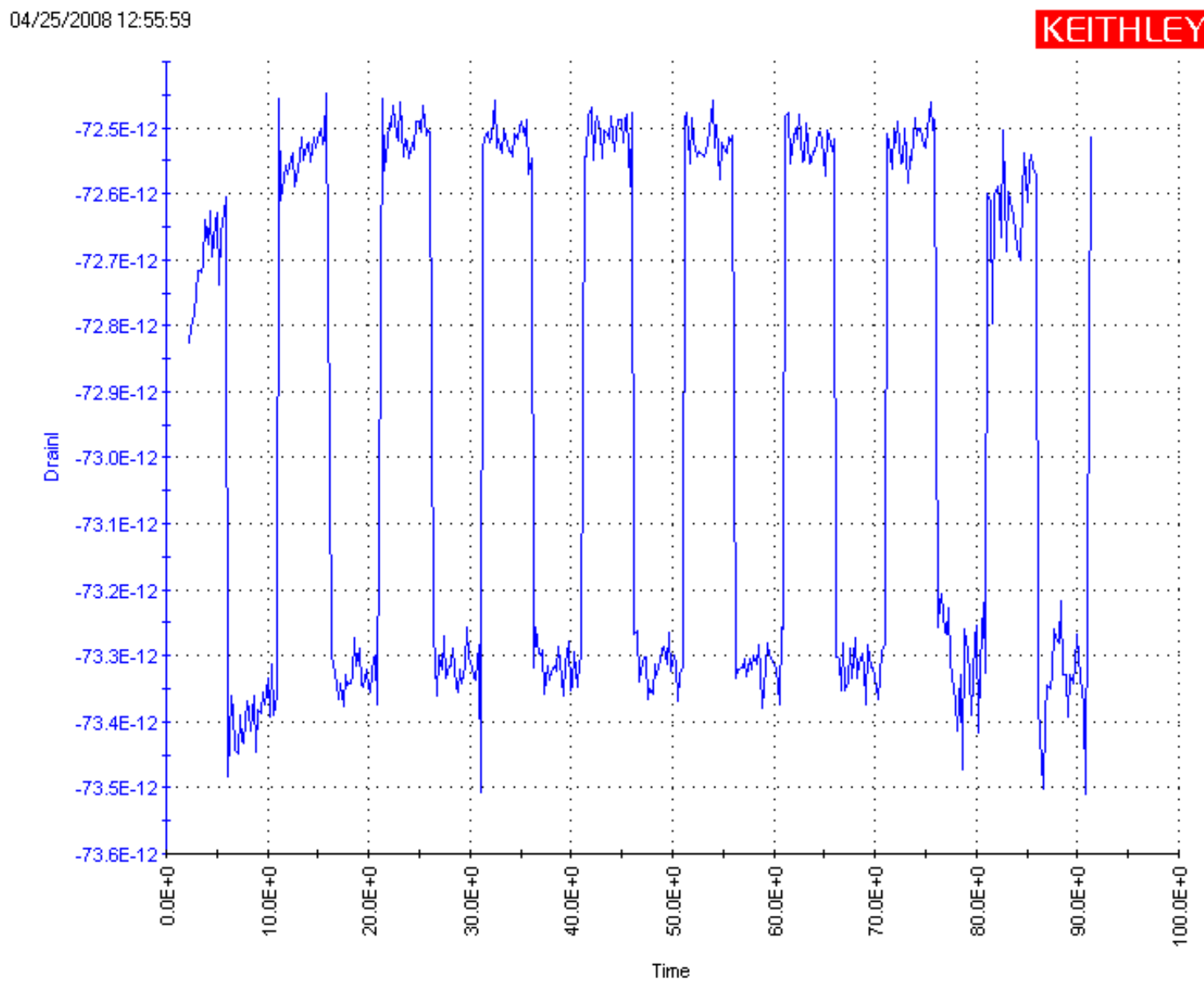

Figure 91: PMOS transistor $(67.5 \mu \mathrm{m} \times 0.81 \mu \mathrm{m})$ response (drain current vs. time) to a $30 \mathrm{mV}$ square wave run through the measurement setup described in this section. Time divisions $=10$ seconds, current divisions $=0.1 \mathrm{pA}$

The square waves produced by the function generator resulted in square shaped responses from the PMOS transistor. This result indicated that the sloped responses due to transistor activation by the PM photovoltage were due to the shape of the PM response to light, and not to the measurement setup.

Remote activation of a PMOS transistor by a patterned PM film was proven successful with the addition of an isolating op-amp. This result demonstrated the successful completion of the main goal of this work, to integrate a patterned PM film 
with a CMOS device to create a light detection system. The experiments discussed in this section show the importance of impedance matching in the development of a sensor platform based on PM films. A small impedance matching circuit could be incorporated into the design of a substrate utilizing patterned PM films as the light detection element.

\subsection{PM Integrated Transistor Response vs. Light Intensity}

After the successful demonstration of transistor activation by a remote patterned PM substrate, a study investigating the correlation between the light intensity the PM is exposed to versus the PMOS transistor response to the PM photovoltage input was conducted. The $3.5 \mathrm{~mm}$ square patterned PM film discussed in Section 5.3 was again used in this experiment. The experimental setup shown in Figure 88 was also used to conduct this experiment. The LED light source was positioned $68 \mathrm{~mm}$ from the PM sample for the first set of data, and $300 \mathrm{~mm}$ from the PM sample for the second set of data. The distance between the light and the PM film was changed to reduce the light intensity reaching the PM film. The current to the LED array was decreased after each set of measurements was taken. The LED pulsing circuit used in Section 5.4 was used again for this experiment. The PMOS transistor $(67.5 \mu \mathrm{m} \times 67.5 \mu \mathrm{m})$ was given a drain voltage of -2.0 volts and a body bias of -0.4 volts for all measurements taken during this experiment. Oscilloscope measurements were taken in DC mode.

The PM film was exposed to 14 different lighting conditions during this study. Photovoltage measurements taken by the oscilloscope and transistor drain current measurements taken by the SPA were captured simultaneously for each lighting condition. The PM film was exposed to a total of 8 different LED currents at a distance

of $68 \mathrm{~mm}$, and 6 different LED currents at a distance of $300 \mathrm{~mm}$. The photovoltage and transistor response graphs for the first and last set of measurements at each distance are shown in Figure 92 thru Figure 99. 


\section{PM Photovoltage}

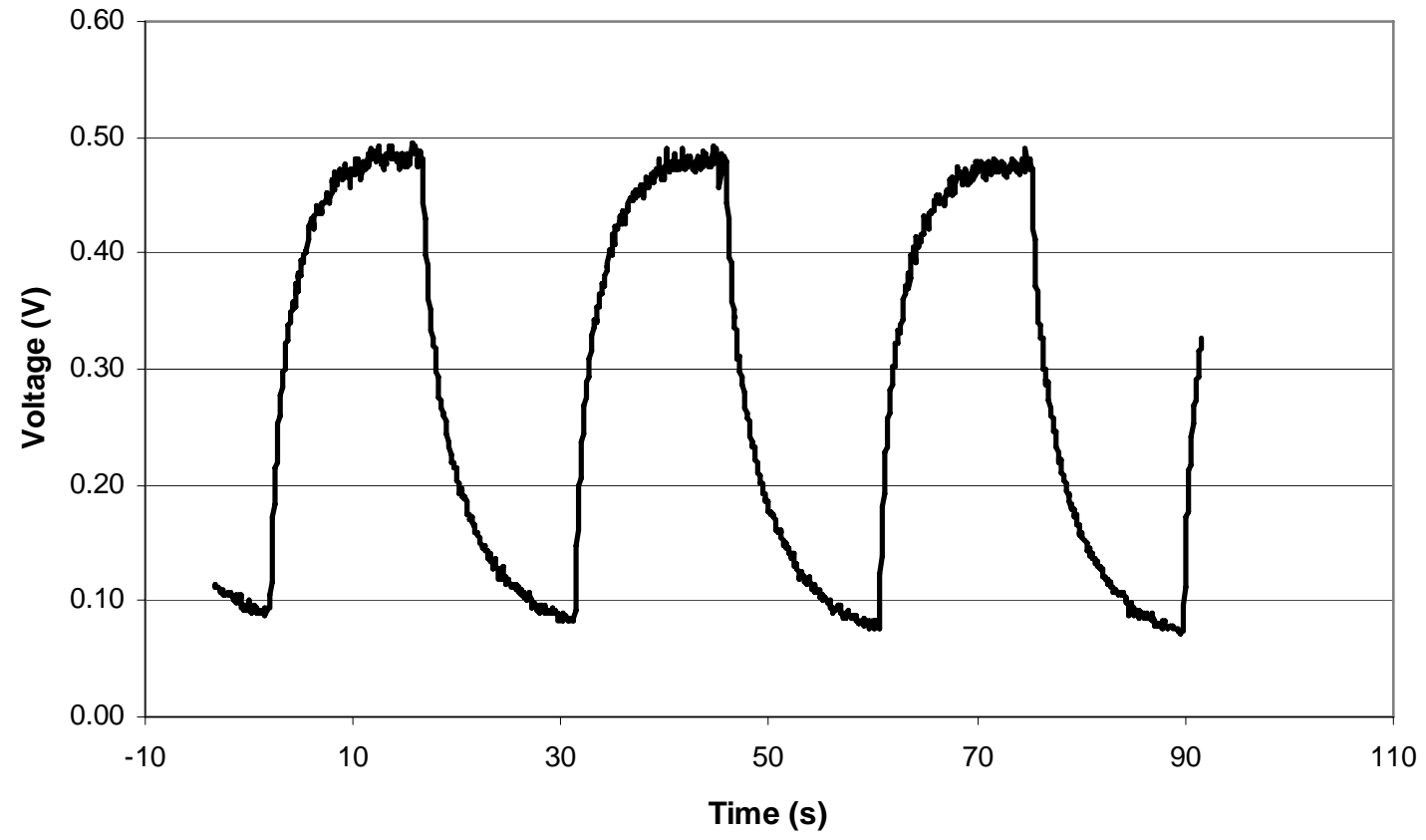

Figure 92: PM photovoltage due to LED illumination of $1.30175 \mathrm{E}-03 \mathrm{~W} / \mathrm{cm}^{2}$ at a distance of $68 \mathrm{~mm}$. A three point moving average was used to smooth the data. 


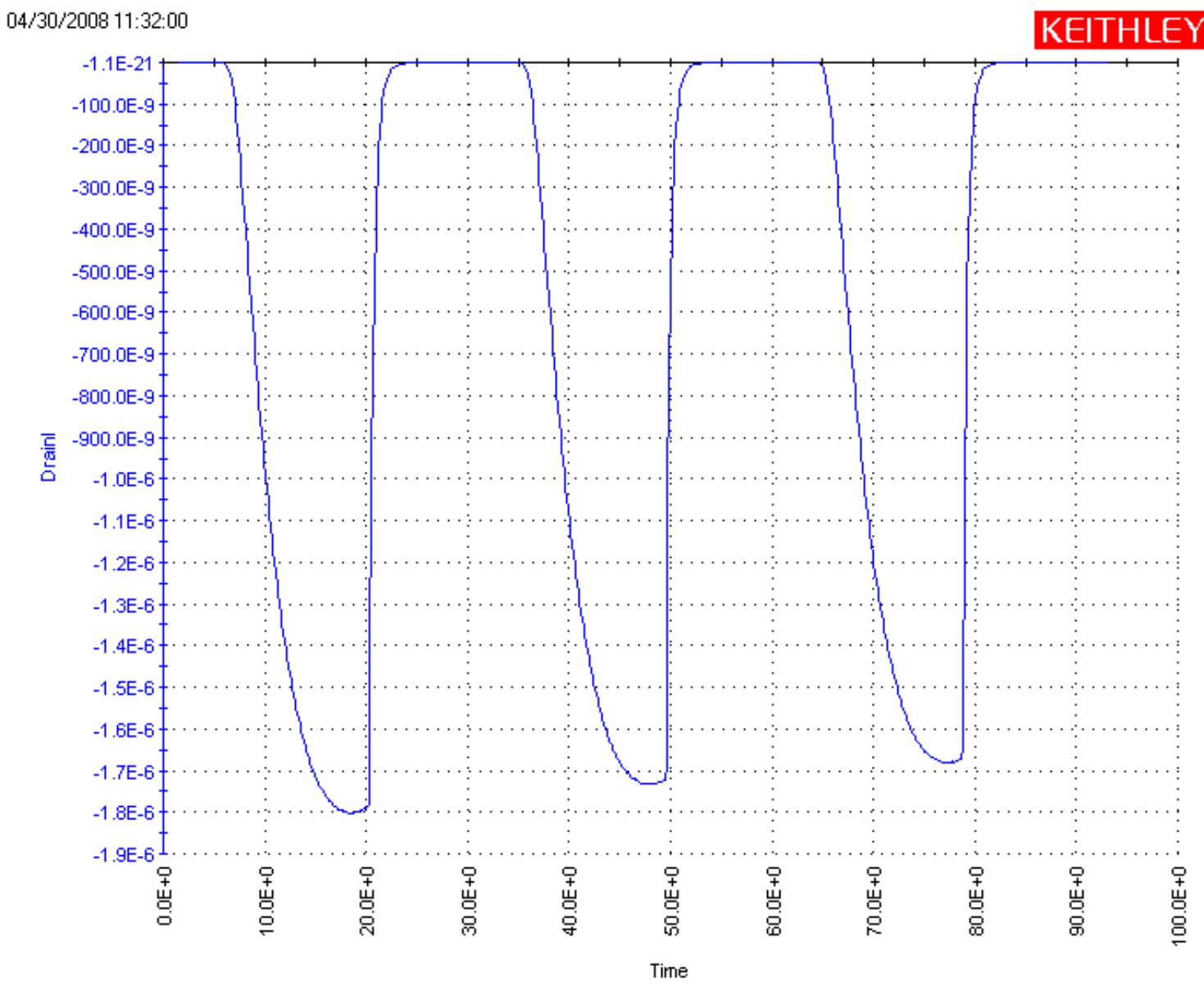

Figure 93: PMOS transistor (67.5 $\mu \mathrm{m}$ x $67.5 \mu \mathrm{m})$ response (drain current) due to PM photovoltage. LED light source produced an optical power of $1.30175 \mathrm{E}-03 \mathrm{~W} / \mathrm{cm}^{2}$ at a distance of $68 \mathrm{~mm}$. Time division $=10$ sec., Drain current division $=100 \mathrm{nA}$. 
PM Photovoltage

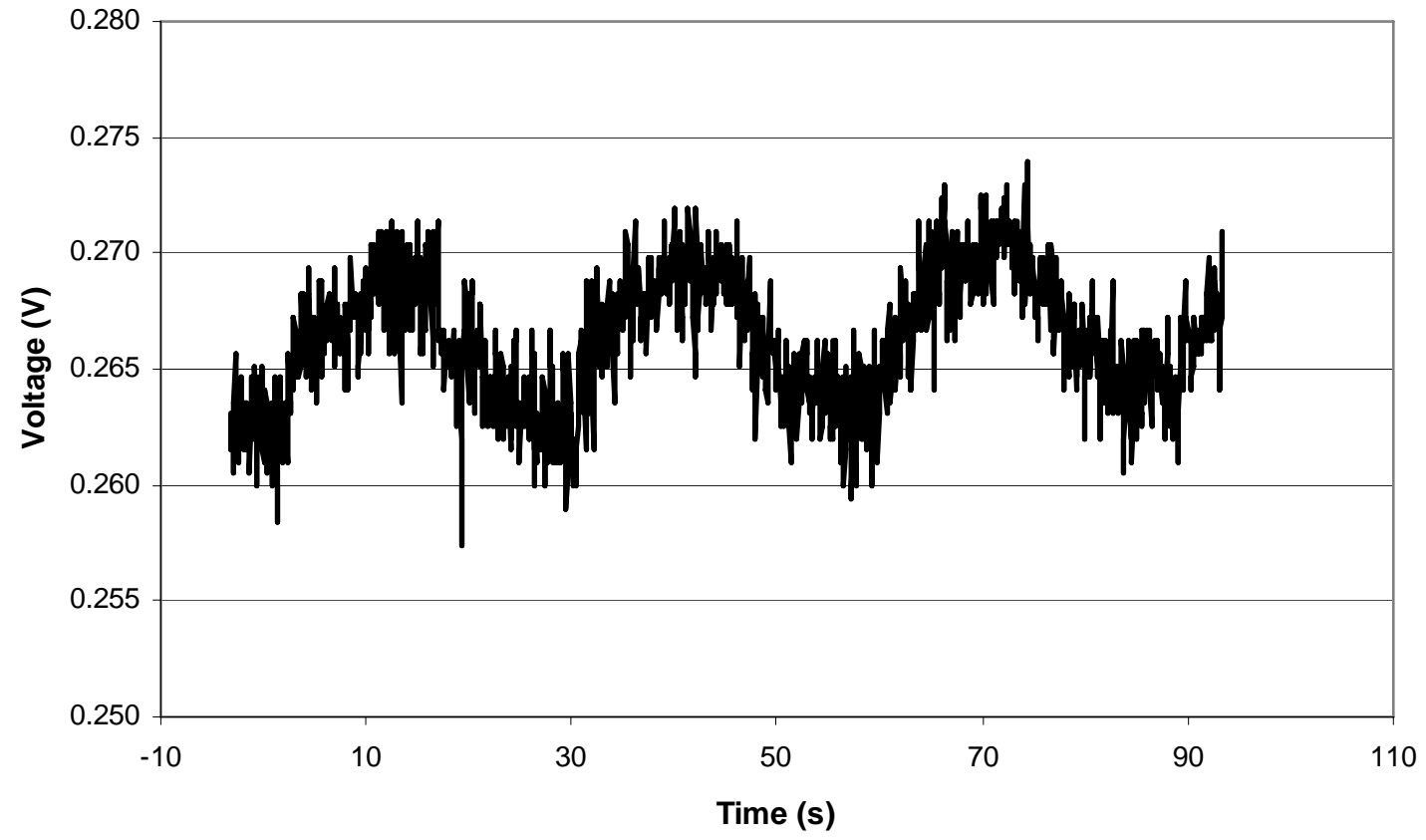

Figure 94: PM photovoltage due to LED illumination of $1.15327 \mathrm{E}-05 \mathrm{~W} / \mathrm{cm}^{2}$ at a distance of $68 \mathrm{~mm}$. A three point moving average was used to smooth the data. 


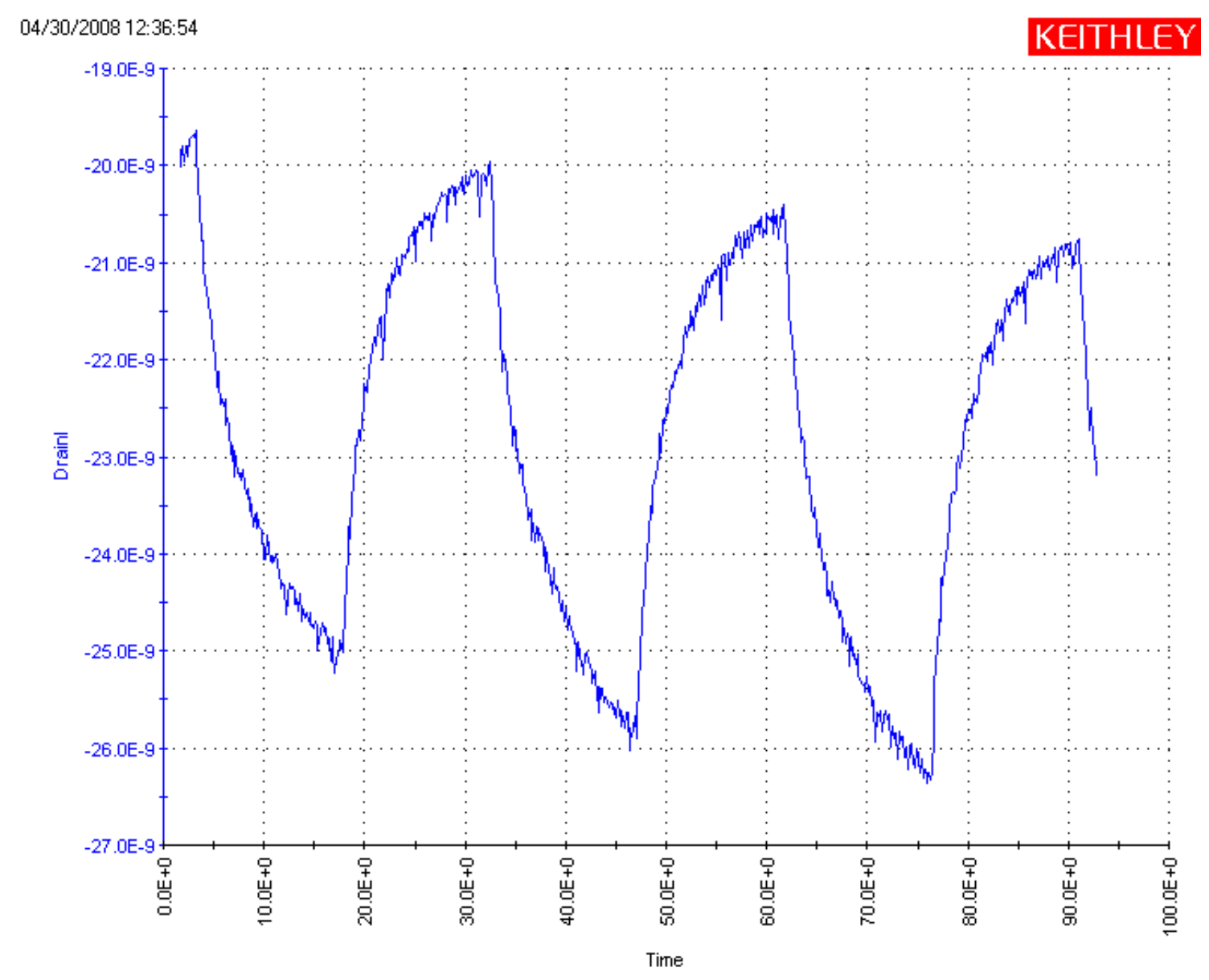

Figure 95: PMOS transistor $(67.5 \mu \mathrm{m} \times 67.5 \mu \mathrm{m})$ response (drain current) due to PM photovoltage. LED light source produced an optical power of $1.15327 \mathrm{E}-05 \mathrm{~W} / \mathrm{cm}^{2}$ at a distance of $68 \mathrm{~mm}$. Time division $=10$ sec., Drain current division $=1.0 \mathrm{nA}$. 


\section{PM Photovoltage}

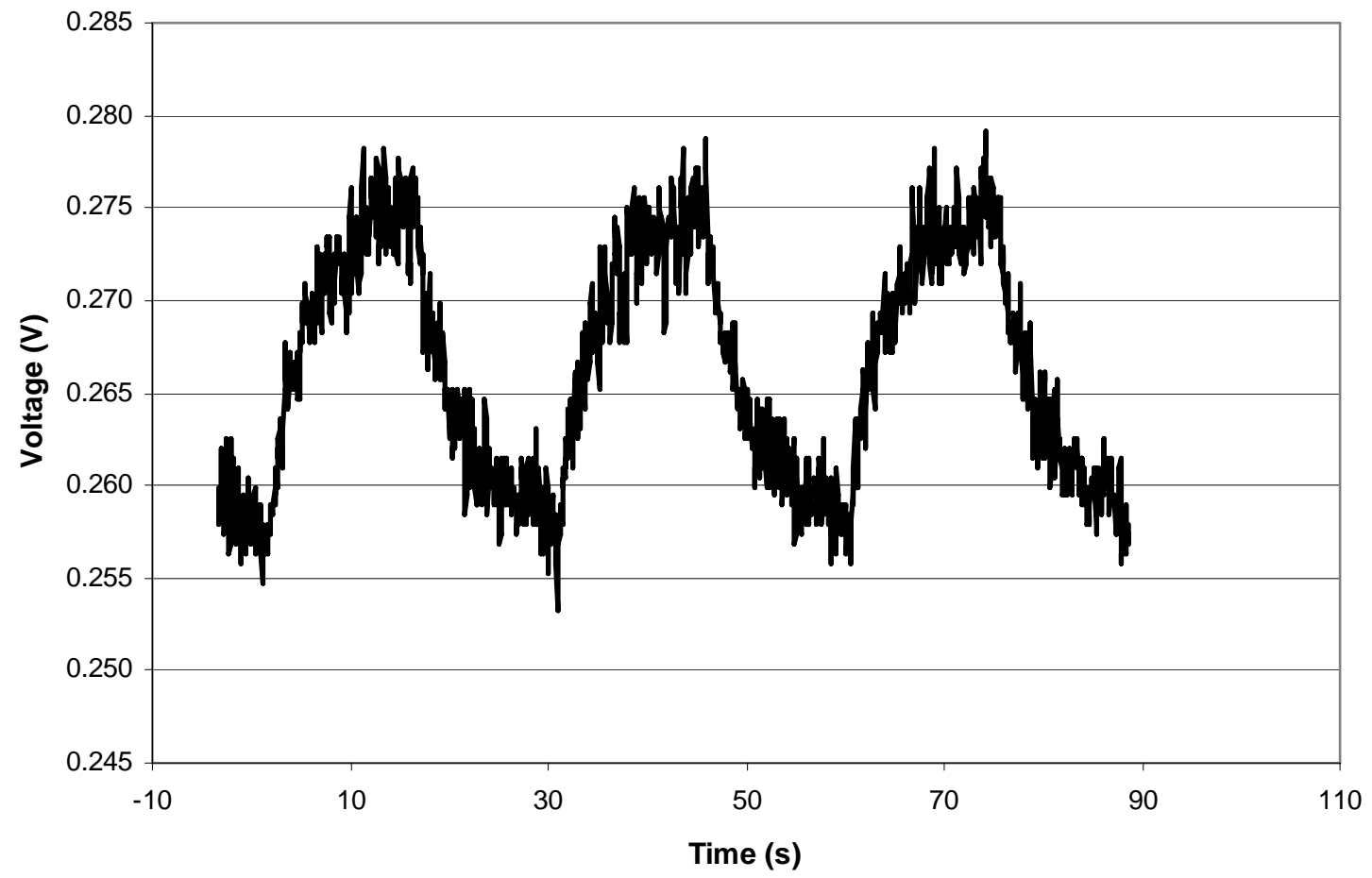

Figure 96: PM photovoltage due to LED illumination of $4.37520 \mathrm{E}-05 \mathrm{~W} / \mathrm{cm}^{2}$ at a distance of $300 \mathrm{~mm}$. A three point moving average was used to smooth the data. 


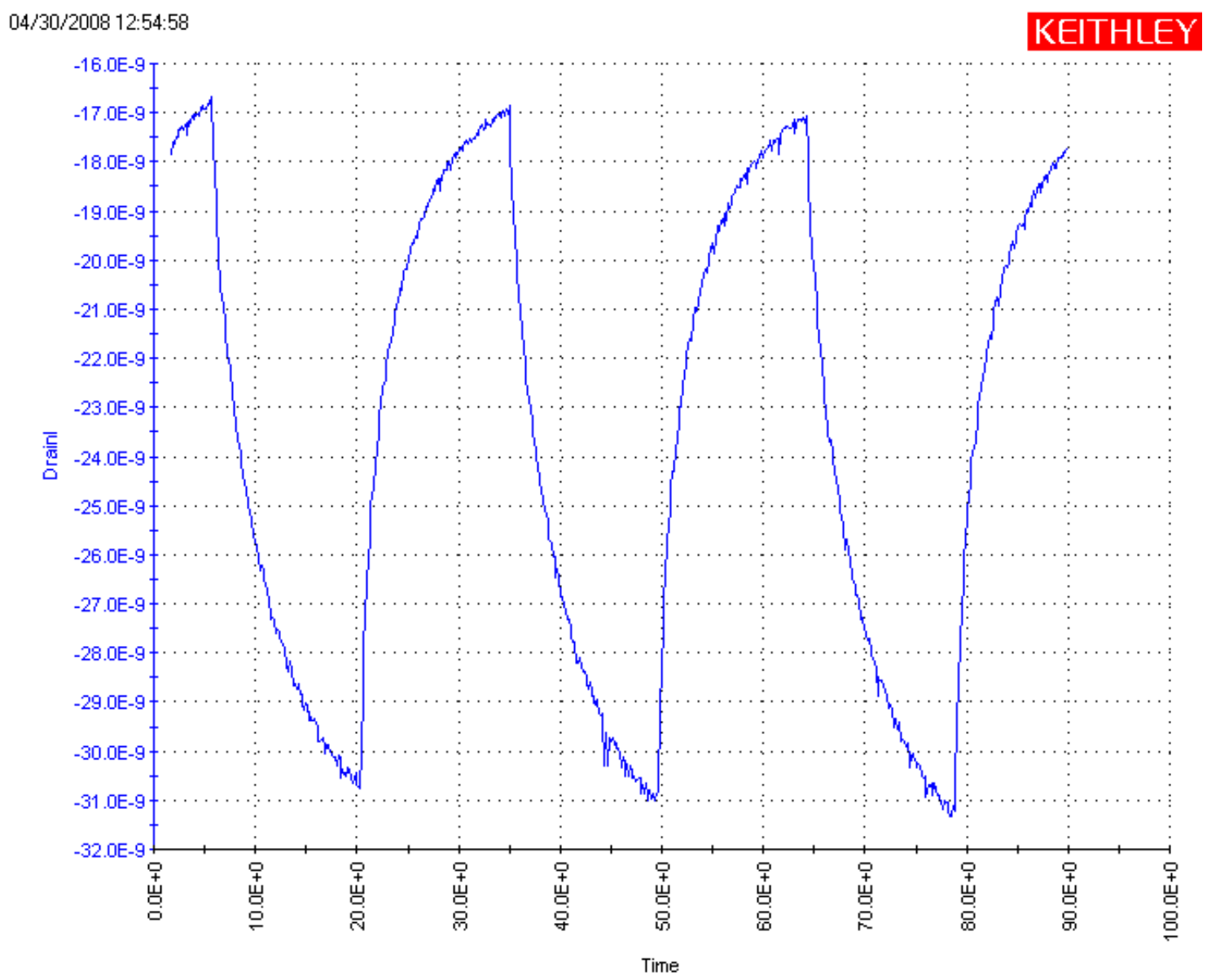

Figure 97: PMOS transistor (67.5 $\mu \mathrm{m}$ x $67.5 \mu \mathrm{m})$ response (drain current) due to PM photovoltage. LED light source produced an optical power of $4.37520 \mathrm{E}-05 \mathrm{~W} / \mathrm{cm}^{2}$ at a distance of $300 \mathrm{~mm}$. Time division $=$ 10 sec., Drain current division $=1.0 \mathrm{nA}$. 
PM Photovoltage

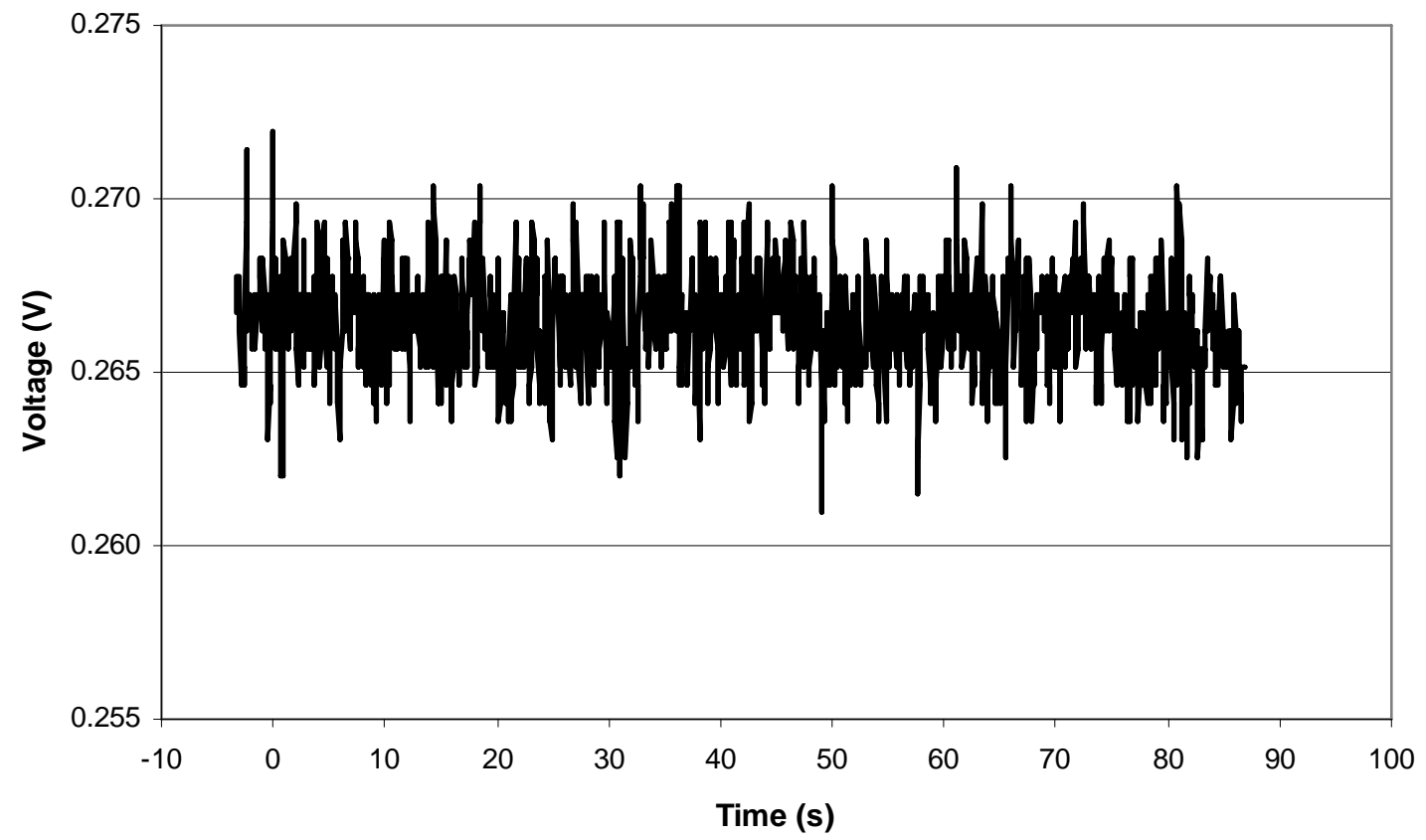

Figure 98: PM photovoltage due to LED illumination of $8.79365 \mathrm{E}-07 \mathrm{~W} / \mathrm{cm}^{2}$ at a distance of $300 \mathrm{~mm}$. A three point moving average was used to smooth the data. 


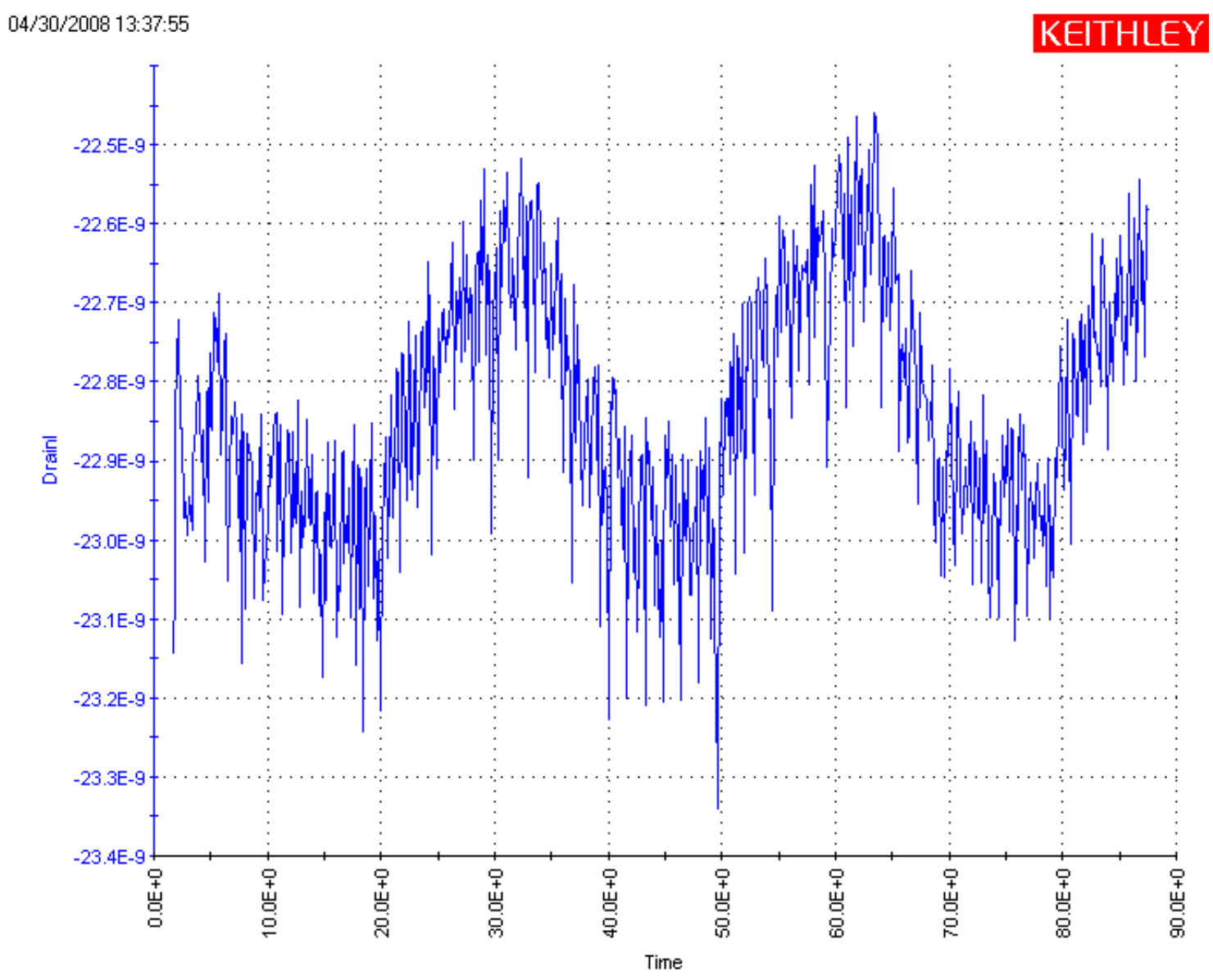

Figure 99: PMOS transistor (67.5 $\mu \mathrm{m} \times 67.5 \mu \mathrm{m})$ response (drain current) due to PM photovoltage. LED light source produced an optical power of $8.79365 \mathrm{E}-07 \mathrm{~W} / \mathrm{cm}^{2}$ at a distance of $300 \mathrm{~mm}$. Time division = 10 sec., Drain current division $=0.1 \mathrm{nA}$.

As the LED light intensity was reduced by decreasing the LED current, both the photovoltage produced by the PM and the response of the PMOS transistor were reduced. As seen in the previous section, the shape of the transistor response mirrors the shape of the PM photovoltage measured using the oscilloscope. An overview of the full results from this study was compiled, and is shown in Table 5. The LED current was measured using a Fluke 187 True RMS multimeter, and is reported in the first column. The distance column shows the separation distance between the PM film and the LED array. The intensity of light produced by the LED array under each current and distance 
combination was measured at a wavelength of $570 \mathrm{~nm}$ using a Thor Labs PM100 optical power meter. The average optical power measurement captured using the optical power meter was divided by the area of the detector, and the optical power was reported in watts $/ \mathrm{cm}^{2}$.

PM photovoltage was approximated by looking at the smoothed graphs produced for each lighting condition using excel. The low voltage value from two valleys were averaged and subtracted from the peak voltage in between the valleys. The transistor response was approximated using the current values provided by the SPA. In this case the average value of two peaks were subtracted from the valley in between them to get an average current response. The averaging was performed in order to reduce the impact of signal drift on the measured responses of the PM film and transistor. Plots of light intensity versus PM photovoltage and light intensity versus transistor response are shown in Figure 100 and Figure 101.

Table 5: Summary of the results from the PMOS transistor response vs. LED light intensity study.

\begin{tabular}{|c|c|c|c|c|}
\hline $\begin{array}{c}\text { LED } \\
\text { Current } \\
\text { (A) }\end{array}$ & $\begin{array}{c}\text { Distance } \\
\text { (mm) }\end{array}$ & $\begin{array}{c}\text { Light Intensity } \\
\left(\mathrm{W} / \mathrm{cm}^{2}\right)\end{array}$ & $\begin{array}{c}\text { PM Photovoltage } \\
\text { (V) }\end{array}$ & $\begin{array}{c}\text { Transistor Response } \\
\text { (A) }\end{array}$ \\
\hline 0.075 & 68 & $1.30175 \mathrm{E}-03$ & 0.4 & $1.80 \mathrm{E}-06$ \\
\hline 0.05 & 68 & 7.87824E-04 & 0.29 & $6.50 \mathrm{E}-07$ \\
\hline 0.04 & 68 & 5.91048E-04 & 0.24 & $3.60 \mathrm{E}-07$ \\
\hline 0.03 & 68 & $3.89227 \mathrm{E}-04$ & 0.165 & $1.80 \mathrm{E}-07$ \\
\hline 0.02 & 68 & $2.20562 \mathrm{E}-04$ & 0.095 & $4.50 \mathrm{E}-08$ \\
\hline 0.0088 & 68 & 6.58803E-05 & 0.03 & $1.50 \mathrm{E}-08$ \\
\hline 0.005 & 68 & 2.52277E-05 & 0.0175 & 1.10E-08 \\
\hline 0.0025 & 68 & 1.15327E-05 & 0.007 & 5.00E-09 \\
\hline 0.029 & 300 & $4.37520 \mathrm{E}-05$ & 0.0175 & $1.40 \mathrm{E}-08$ \\
\hline 0.0185 & 300 & 2.30653E-05 & 0.008 & 6.50E-09 \\
\hline 0.0115 & 300 & 1.17489E-05 & 0.005 & $3.25 \mathrm{E}-09$ \\
\hline 0.0051 & 300 & 4.58423E-06 & 0.002 & 1.20E-09 \\
\hline 0.0024 & 300 & 1.60736E-06 & 0.0005 & $5.25 \mathrm{E}-10$ \\
\hline 0.0015 & 300 & 8.79365E-07 & 0 & $3.75 \mathrm{E}-10$ \\
\hline
\end{tabular}


PM Photovoltage vs. Light Intensity

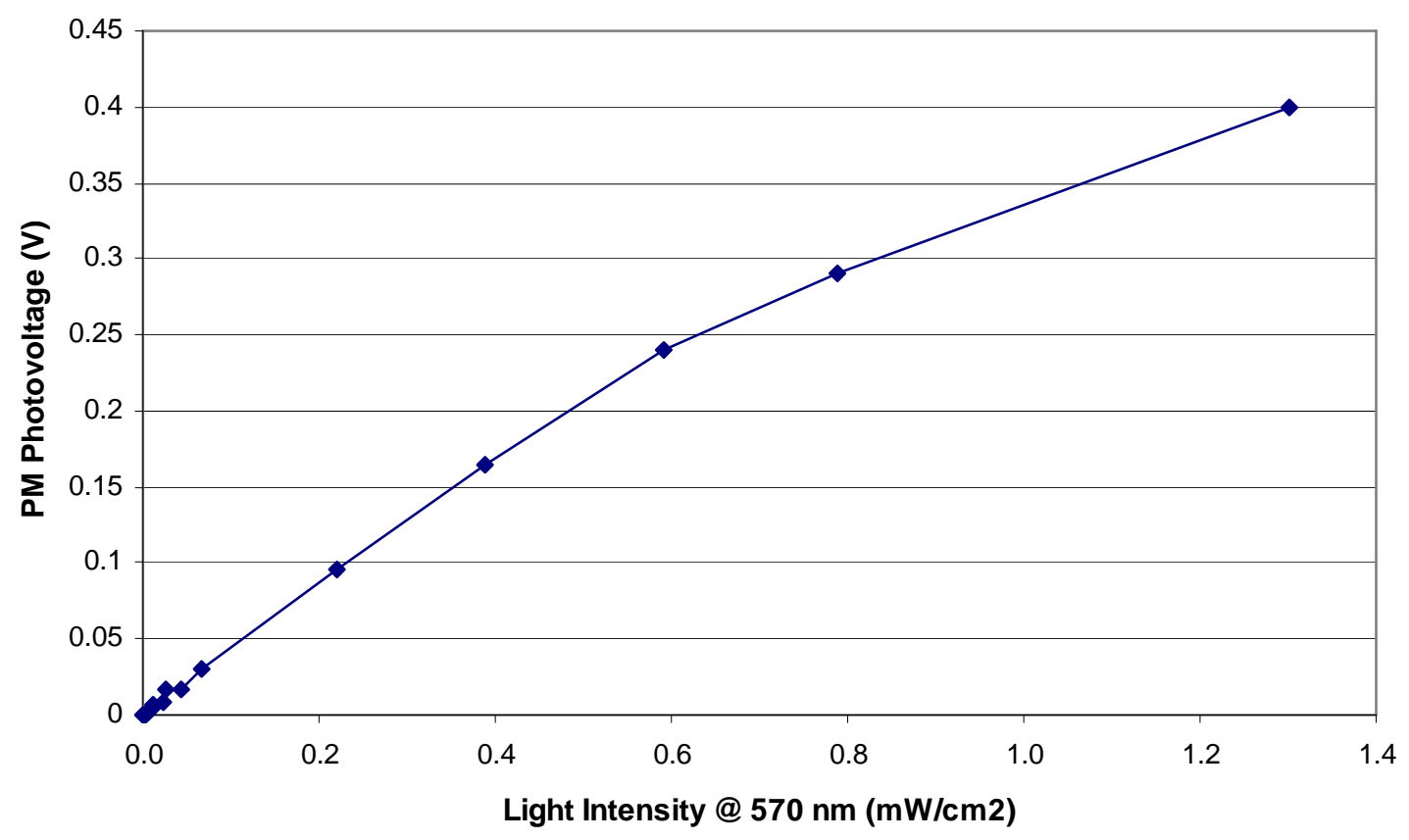

Figure 100: Approximate maximum PM photovoltage versus LED light intensity. 


\section{Transistor Response vs. Light Intensity}

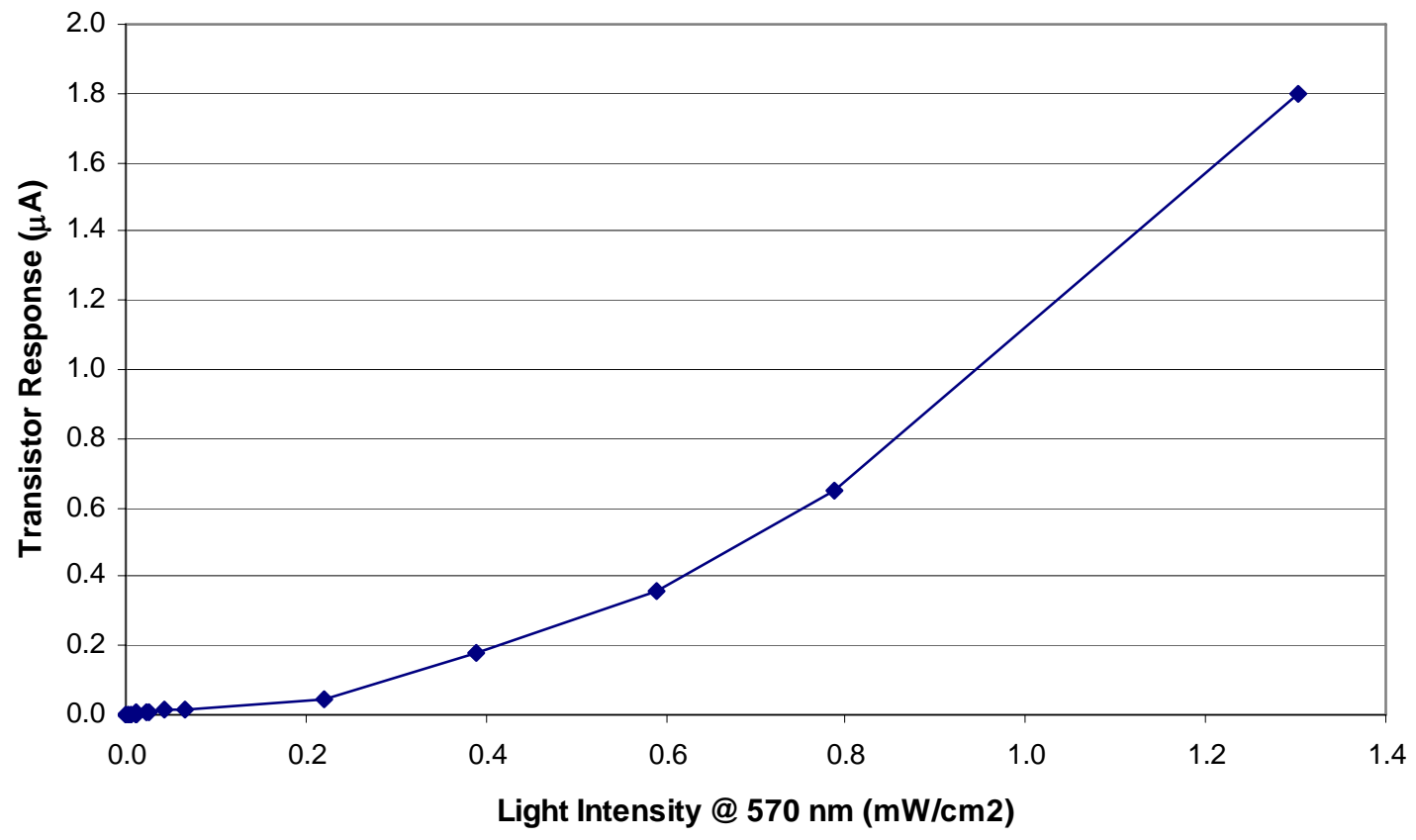

Figure 101: Approximate maximum PMOS transistor drain current response versus LED light intensity.

Figure 100 and Figure 101 show a consistent upward trend in both the PM photovoltage and the gate current of the transistor as the LED light intensity was increased. Figure 100 shows a roughly linear trend, with some reduction in the slope of the trend taking place at the last two data points. This slope reduction could be due to some portion of the bR molecules in the patterned PM film reaching saturation. Figure 101 shows a non-linear trend, with increased light intensities resulting in larger increases in transistor gate currents. This trend is expected due to the non-linear response characteristics of the PMOS transistor used in this experiment.

This experiment has demonstrated the effectiveness of coupling PM films with MOSFET transistors. As the lighting conditions that the PM film is exposed to change, both the produced photovoltage and the transistor response change. This experiment has 
also shown that the patterned PM film was able to produce a photovoltage detectible by the PMOS transistor under very low light intensities. No detailed experiments investigating the impact of light intensity on the photovoltage produced by PM were found in the literature. This information will be necessary when a purpose built system integrating PM films and microelectronics is designed. 


\subsection{Summary and Conclusions}

The goal of this research was to develop a method for patterning oriented PM films onto electronic substrates, and to use this patterning technique to integrate PM films with CMOS devices. The patterning technique must be proven capable of producing patterned PM films that retain photoelectric functionality. The technique must also be compatible with sensitive CMOS substrates. After the development of an appropriate patterning technique, patterned PM films should be integrated with a microelectronic device such as a MOSFET to provide a proof of concept for further system level work in this area.

Purified PM films were produced in house to obtain the highest quality material possible for use in this research. The batch growth and purification process outlined in Chapter 2 yielded PM preparations with greater than 90\% purity. Early development of a novel FIB patterning technique proved impractical for large scale patterning of PM films. Following this discovery, the PBBPT was developed. This lithography based patterning technique proved capable of patterning PM films onto flat substrates while retaining the photoelectric activity of the PM films. Because the PBBPT is based on lithographic processing, the process is highly compatible with electronic substrates. This process could easily be integrated after a microelectronic device fabrication process flow to produce devices functionalized with patterned PM films.

Several PBBPT process variables were studied and optimized, including the PM suspension density during electrodeposition, as well as acetone sonication time. A PM suspension density of $0.908 \mathrm{mg} / \mathrm{ml}$ or larger produced quality patterned films. The optimal sonication time under the experimental conditions described in Section 4.2.2 was 60 seconds, with 45 seconds leading to incomplete patterning and 120 seconds leading to the damage or complete removal of patterned PM films. Quality patterned PM films were produced with feature sizes down to $15 \mu \mathrm{m}$, and irregular films were produced 
down to a feature size of $5 \mu \mathrm{m}$. A temperature study conducted on patterned PM films indicated that the electrical output of the PM films due to light exposure was rapidly reduced as temperatures exceed $80^{\circ} \mathrm{C}$, and a complete loss in electrical activity was seen at $120^{\circ}-130^{\circ} \mathrm{C}$. These studies have allowed for the PBBPT to be optimized, which has resulted in higher quality films. The results from the temperature study also provide some baseline information on the maximum temperature that this sensing platform could withstand and remain a functional system.

Variations in the PBBPT process were developed and investigated. These variations allowed for PM films to be deposited onto non-conductive substrates, and also for thin films to be deposited on top of the PM films to act as a top electrode for electrical measurement purposes. These two process variations greatly increased the versatility of the PBBPT process. The process can now be used on top of pre-constructed microelectronics, making rapid integration of patterned PM films with microelectronic devices possible. The ability to electrically contact the top of the PM film without compromising its photoelectric properties allow for the PM film to be referenced to a common ground shared by the microelectronic device. This may be vital to designing a working system that integrates PM films with transistors or other microelectronic devices.

Following the development and optimization of the PBBPT process, patterned films were integrated with individual PMOS transistors. Initial studies involved patterning PM films directly onto the MOSFET substrate containing the transistors. This proved unsuccessful, as the light used to activate the PM films also activated the transistors on the substrate. In order to avoid transistor activation due to light, PM was patterned onto a separate substrate, and the photovoltage produced by the PM film when exposed to a light source was transferred to the gate of a PMOS transistor through co-axial probes. Due to an impedance mismatch between the PM film and the PMOS transistor, the PM signal was passed through a high impedance operational amplifier. This experimental 
setup allowed the PM photovoltage to activate the PMOS transistor, demonstrating successful integration of a patterned PM film with a CMOS transistor. A further study using this setup demonstrated the dependence of both PM photovoltage output and transistor drain current on the intensity of light the PM film was exposed to. This study provided insight into the amount of light (optical power) required for the PM to produce a photovoltage measurable to the PMOS transistor.

Through the many processes and studies performed during the course of this research, the goals of this research have been accomplished. The PBBPT was developed, which provides a method for patterning PM films onto electronic substrates. The successful integration of PM films with PMOS transistors was also demonstrated. These results are critical in reaching the overall goal of this project, the development of a PM based toxin detection platform. Work currently underway and future work by colleagues will build upon the techniques and results described here to design and fabricate a miniaturized detection system using PM films as an optical to electrical transduction source.

\subsection{Suggestions for Future Work}

This research represents the initial efforts towards the realization of a miniaturized toxin detection platform that will utilize PM films as one component in the sensing system. Now that the groundwork has been laid for the patterning of PM films onto electronic substrates, further research is needed to successfully integrate PM films into a detection platform. The following are research areas that would build off this work to help realize a PM based miniaturized light detection platform:

Develop a customized microelectronic platform that would allow for arrays of PM sensing sites to be integrated with electronic devices such as field effect transistors. The

platform should address the issues of shielding the transistors from light as well as compensating for any impedance mismatch between the PM and the electronic devices. 
Several additional issues must be addressed before a platform can be designed. Studies must be conducted to determine how different characteristics of the patterned PM film (lateral dimensions, thickness, metallic top coating) affect the photoelectric response of the film. This information must then be used to determine the optimal PM sensing site size to activate the selected microelectronic device. The minimum surface area required for successful target sensing should also be considered when designing the PM sensing site size.

Modify the PBBPT to utilize other PM film deposition methods that would allow for thin films of PM to be patterned. Deposition methods other than electrodeposition should be considered when integrating patterned PM films with microelectronic devices. The precise PM layer control made possible by the LB and ELBL deposition methods may prove advantageous when attempting to fabricate reliable and reproducible PM sensing sites. The use of sensitive microelectronic devices may allow the photovoltage produced by PM films 10's of nanometers thick to be detected. After the PBBPT process is successfully modified and optimized for these more precise deposition methods, the integration of thinner patterned PM films both directly and indirectly with microelectronic devices should be performed.

A comprehensive study investigating the degree of orientation of PM films deposited using electrodeposition, LB, and ELBL techniques should be performed. Currently there is no experimental data that quantifies the effectiveness of different PM deposition techniques. Understanding the degree of PM orientation produced using these deposition techniques will be critical to producing the best possible PM based detection system. A novel experiment must be designed and carried out in order to obtain this valuable information.

Investigate the use of bR monomers in conjunction with the PBBPT. One factor that may limit the minimum feature size currently attainable through the PBBPT is the size of 
the individual PM patches being deposited. It is possible to remove the individual bR molecules from the PM patches, leaving a suspension of bR molecules. The bR molecules do not need the structure or the lipid content of the PM film in order to function. Work should be done to develop techniques for depositing and patterning bR monomers using the PBBPT. Through this research, it may be possible to reduce the minimum feature size obtained through the PBBPT, as well as increase the electrical output per volume when compared to PM patterned films.

Conduct real time temperature variation studies to determine how the output of the PM is affected by changes in temperature, and how these changes may impact the functionality of a PM based detection system. To accomplish this, a test setup must be developed that will allow a substrate containing a patterned PM film to be heated without introducing noise into the measurement setup. Previous studies have shown that placing a thermocouple probe inside the grounded box containing a patterned PM film produced a large amount of electrical noise. It may be necessary to use some other means of temperature detection to monitor the substrate temperature while measurements are taken. It must also be confirmed that whatever is used to heat the substrate does not influence the electrical measurements.

Perform long-term PM photoelectric activity tests to ascertain the stability of a PM film's photovoltage over time. Several separate studies should be conducted that address various aspects of long term PM film photovoltage. A study should be performed in which a patterned PM film integrated with a microelectronic device is exposed to a constant light source for a long period of time (days to weeks). After a set period of time, the light source should be pulsed, and the effect of the light pulsing on the output of the microelectronic device should be measured. The magnitude of this change should then be compared to the photovoltage produced by the same film due to identical light pulsing prior to prolonged exposure to light. A second study should be performed in which the electrical response of a patterned PM film due to pulsed light is recorded once a month 
for several months. The PM film should not be exposed to light in between photovoltage tests. These two studies will provide data on the long term stability of the photoelectric response of a patterned PM film under both constant illumination and constant darkness. 


\subsection{References}

[1] Hampp, N., "Bacteriorhodopsin as a Photochromic Retinal Protein for Optical Memories,” Chem. Rev., vol. 100, P. 1755-1776, 2000

[2] Crittenden, S., Howell, S., Reifenberger, R., Hillebrecht, J., Birge, R.R., "Humidity-Dependent Open-Circuit Photovoltage from a Bacteriorhodopsin/indium Tin Oxide Bioelectronic Heterostructure,” Nanotechnology, vol. 14, P. 562-565, 2003

[3] Shen, Y., Safinya, C., Liang, K.S., Rupert, A.F., Rothschild, K.J., "Stabilization of the Membrane Protein Bacteriorhodopsin to $140{ }^{\circ} \mathrm{C}$ in TwoDimensional Films” Nature, vol. 366, P. 45-52, 1993

[4] Chen, Z., Lewis, A., Takei, H., Nebenzahl, I., "Bacteriorhodopsin Oriented in Polyvinyl Alcohol Films as an Erasable Optical Storage Medium,” Applied Optics, vol. 30, P. 5188-5196, 1991

[5] Wang, W.W., Knopf, G.K., Bassi, A.S., "Photoelectric Properties of a Detector Based on Bacteriorhodopsin Films,” Biosensors and Bioelectronics, vol. 21, P. 1309-1319, 2006

[6] Xu, J., Stickrath, A.B., Bhattacharya, P., Nees, J., Varo, G., Hillebrecht, J.R., Ren, L., Birge, R.R., "Direct Measurement of the Photoelectric Response Time of Bacteriorhodopsin via Electro-Optic Sampling,” Biophys. J., vol. 85, P. 1128-1134, 2003

[7] Methot, M., Desmeules, P., Vaknin, D., Boucher, F., Salesse, C., "In Situ Characterization of Functional Purple Membrane Monolayers at the Air-Water Interface,” Langmuir, vol. 20, P. 934-940, 2004

[8] Wang, J.P., Li, J.R., Tao, P.D., Chang, X., Jiang, L., "Photoswitch Based on Bacteriorhodopsin Langmuir-Blodgett Films,” Advanced Materials for Optics and Electronics, vol. 4, P. 219-224, 1994

[9] Ikonen, M., Sharonov, A., Tkachenko, N., Lemmetyinen, H., “The Photovoltage Signals of Bacteriorhodopsin in Langmuir-Blodgett Films with Different Molecular Orientations," Advanced Materials for Optics And Electronics, vol. 2, P. 115-122, 1993 
[10] Miyasaka, T., Koyama, K., "Image Sensing and Processing by a Bacteriorhodopsin-Based Artificial Photoreceptor,” Applied Optics, vol. 32, P. 6371-6379, 1993

[11] Henderson, R., Jubb, J.S., Whytock, S.J., "Specific Labeling of the Protein and Lipid on the Extracellular Surface of Purple Membrane,” J. Mol. Biol., vol. 123, P. 259-274, 1978

[12] Chen, D., Lu, Y., Sui, S., Xu, B., Hu, K., “Oriented Assembly of Purple Membrane on Solid Support, Mediated by Molecular Recognition,” J. Phys. Chem., vol. 107, P. 3598-3605, 2003

[13] Ren, Q., Zhao, Y., Han, L., Zhao, H., “A Nanomechanical Device Based on Light Driven Proton Pumps,” Nanotechnology, vol. 17, P. 1778-1785, 2006

[14] Chrisey, D. B., Hubler, G. K., Pulsed Laser Deposition of Thin Films. New York: Wiley, 1994

[15] Agarwal, G., Phadke, R.S., "Laser Assisted Deposition of Bacteriorhodopsin Assemblies,” Nanotechnology, vol. 10, P. 336-339, 1999

[16] Szoka, F., Papahadjopoulos, D., Liposomes: From Physical Structure to Therapeutic Application. New York: Elsevier/North-Holland Biomedical Press, 1981

[17] Oesterhelt, D., Meentzen, M., Schuhmann, L., "Reversible Dissociation of the Purple Complex in Bacteriorhodopsin and Identification of 13-cis and alltrans-Retinal as its Chromophores,” Eur. J. Biochem., vol. 40, P. 453-463, 1973

[18] Decher, G., Hong, J. D., Schmitt, J., "Buildup of Ultrathin Multilayer Films by a Self-Assembly Process III: Consecutively Alternating Adsorption of Anionic and Cationic Polyelectrolytes on Charged Surfaces," Thin Solid Films, vol. 210/211, P. 831-835, 1992

[19] Raposo, M., Oliveira, O., “Adsorption Mechanisms in Layer-by-Layer Films,” Bras. J. Phys. [online] Vol. 28, no. 4, 1998

[20] He, J., Samuelson, L., Li, L., Kumar, J., Tripathy, S. K., "Oriented Bacteriorhodopsin/Polycation Multilayers by Electrostatic Layer-by-Layer Assembly,” Langmuir, vol. 14, P. 1674-1679, 1998 
[21] He, J.A., Samuelson, L., Li, L., Kumar, J., Tripathy, S.K., "Bacteriorhodopsin Thin Film Assemblies - Immobilization, Properties, and Applications," Adv. Mater, vol. 11, P. 435-446, 1999

[22] Oesterhelt, D., Stoeckenius,W., "Isolation of the Cell Membrane of Halobacterium halobium and its Fractionation into Red and Purple Membrane,” Methods Enzymol., vol. 31, P. 667-678, 1974

[23] He, J., Samuelson, L., Li, L., Kumar, J., Tripathy, S. K., "Photoelectric Properties of Oriented Bacteriorhodopsin/Polycation Multilayers by Electrostatic Layer-by-Layer Assembly,” J. Phys. Chem., vol. 102, P. 70677072, 1998

[24] Li, M., Li, B., Jiang, L., Tussila, T., Tkachenko, N., Lemmetyinen, H., “The Fast Photovoltaic Response from Multilayer by Alternate Layer-by-Layer Assembly of Polycation and Bacteriorhodopsin,” Chem. Lett., vol. 29, P. 266267, 2000

[25] Crittenden, S., Reifenberger, R., Hillebrecht, J., Birge, R., Inerowicz, D., Regnier, F., "Soft Lithography Based Micron-Scale Electrophoretic Patterning of Purple Membrane,” J. Micromech. Microeng. vol. 15, P. 1494-1497, 2005

[26] Fang, A., Ng, H., Su, X., Li, S. F. Y., "Soft-Lithography-Mediated Submicrometer Patterning of Self-Assembled Monolayer of Hemoglobin on ITO Surfaces,” Langmuir, vol. 16, P. 5221-5226, 2000

[27] Hyun, J., Chilkoti, A., "Micropatterning Biological Molecules on a Polymer Surface Using Elastomeric Microwells,” J. Am. Chem., vol. 123, P. 69436944, 2001

[28] Bhattacharya, P., Xu, J., Varo, G., Marcy, D., Birge, R. R., "Monolithically Integrated Bacteriorhodopsin-GaAs Field-Effect Transistor Photoreciever," Optics Lett., vol. 27, P. 839-841, 2002

[29] Takei, H., Lewis, A., Chen, Z., Nebenzahl, I., "Implementing Receptive Fields with Excitatory and Inhibitory Optoelectrical Responses of Bacteriorhodopsin Films,” Applied Optics, vol. 30, No. 4, 1991

[30] Haronian, D., Lewis, A., "Microfabricating Bacteriorhodopsin Films for Imaging and Computing,” Appl. Phys. Lett., vol. 61, P. 2237-2239, 1992

[31] Oesterhelt, D., Stoeckenius, W., "Rhodopsin-Like Protein from the Purple Membrane of Halobacterium halobium,” Nature New Bio., vol. 233, P. 149152, 1971 
[32] Bridgen, J., Walker, I. D., "Photoreceptor Protein from the Purple Membrane of Halobacterium Halobium. Molecular Weight and Retinal Binding Site," Biochem., vol. 15, P. 792-798, 1976

[33] Holman, J. P., Experimental Methods for Engineers. New York: McGrawHill Book Company, 1966. 